November 1991

\title{
LATTICE CALCULATION OF NONLEPTONIC CHARM DECAYS*
}

\author{
James Nicholas Simone ${ }^{* *}$ \\ Physics Department \\ Brookhaven National Laboratory \\ Upton, New York 11973-5000
}

\section{DISCLAIMER}

\begin{abstract}
This report was prepared as an account of work sponsored by an agency of the United States Government. Neither the United States Government nor any agency thereof, nor any of their employees, makes any warranty, express or implied, or assumes any legal liability or responsibility for the accuracy, completeness, or usefulness of any information, apparatus, product, or process disclosed, or represents that its use would not infringe privately owned rights. Reference herein to any specific commercial product, proccss, or service by trade name, trademark, manufacturer, or oiherwise does not necessarily constitute or imply its endorsement, recommendation, or favoring by the United States Government or any agency thereof. The views and opinions of authors expressed herein do not necessarily state or reflect those of the United States Government or any agency thereof.
\end{abstract}

* A dissertation submitted to the University of California, Los Angeles, in partial satisfaction of the requirements for the degree of Doctor of Philosophy in Physics (1991).

** Research supported by the U.S. Department of Energy under contract DE-AC02$76 \mathrm{CH} 00016$. 


\section{Contents}

List of Figures ......................... vi

List of Tables ...................... $x$

Acknowledgments .......................... xi

Abstract of the nissertation .................... xij

Chapter 1. Introduction .................. 1

Chapter 2. Lattice Fundamentals . . . . . . . . . . . . . 7

2.1. Continuum Lagrangian . . . . . . . . . . . . . . . 7

2.2. The Lattice Gauge Action . . . . . . . . . . . . . 8

2.3. The Continuum Limit . . . . . . . . . . . . . . . . . 10

2.4. Euclidean Dirac Matrices . . . . . . . . . . . . 13

2.5. Wilson Fermion Action . . . . . . . . . . . . . 13

2.6. Discrete Symmetries of Quark Propagators . . . . . . . . . . 15

2.6.1. Hermiticity . . . . . . . . . . . . . . 15

2.6.2. Charge Conjugation .............. 16

2.6.3. Parity .................. . . 16

2.6.4. Time Inversion . . . . . . . . . . . . . . . . . 17

2.7. Expectation Values . . . . . . . . . . . . . . . . . 18 
Chapter 3. Weak Interaction Fundamentals . . . . . . . . . . . . . . 20

3.1. Motivation .................... 20

3.2. The Continuum Effective weak Hamiltonian _. . . . . . . . 21

3.3. Lattice Effective Weak Hamiltonian . . . . . . . . . . . . . 28

Chapter 4. Lattice Correlation Functions . . . . . . . . . . . . . 34

4.1. Interpolating Fields . . . . . . . . . . . . . . . . 34

4.2. Pseudoscalar Two-Point Functions . . . . . . . . . . . . . 36

4.3. Scalar Two-Point Functions . . . . . . . . . . . . . . . 40

4.4. Extracting Matrix Elements From Four-Point Func-

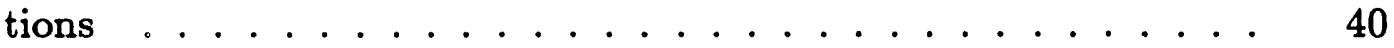

4.5. Lattice Four-point Functions For $D \rightarrow K \pi$. . . . . . . . . 49

4.5.1. $\quad D^{0} \rightarrow K^{-} \pi^{+}$Four-point Functions . . . . . . . . . . 53

4.5.2. $D^{+} \rightarrow \bar{K}^{0} \pi^{+}$Four-point Functions . . . . . . . . . . . 54

4.5.3. $\quad D^{0} \rightarrow \bar{K}^{0} \pi^{0}$ Four-point Functions . . . . . . . . . . . 56

4.5.4. $\quad D \rightarrow K \pi$ Lattice Diagrams and Large N . . . . . . . 58

4.5.5. Lattice Vacuum Saturation Functions . . . . . . . . . . 61

4.5.6. $\quad D^{0} \rightarrow \kappa(\mathrm{d} s \mathrm{scalar}) \ldots . . . . . . . . . .666$

4.6. Dimensionful Four-point Functions . . . . . . . . . . . . . 68

4.7. Discrete Symmetries . . . . . . . . . . . . . . 70

4.7.1. Application to Meson Propagators . . . . . . . . . . . 71 


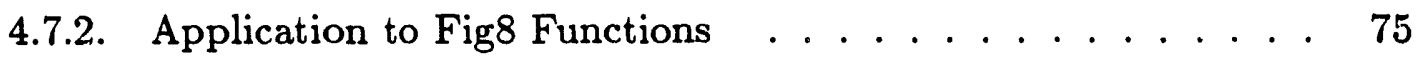

4.7.3. Application to VS Functions $\ldots \ldots \ldots \ldots \ldots$

4.7.4. Application to fig8scalar functions $\ldots \ldots \ldots$. . . . . 81

4.8. Continuum Functions $\ldots \ldots \ldots \ldots \ldots$

4.8.1. Continuum Matrix Elements _. . . . . . . . . . 82

4.8.2. Vacuum Saturation . . . . . . . . . . . 84

Chapter 5. Results .................. 86

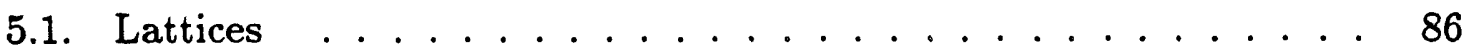

5.2. Propagators and $\chi^{2}$ Fits $\ldots \ldots \ldots \ldots \ldots$

5.3. Time Dependence of Amplitudes _. . . . . . . . . 98

5.4. $\mathcal{A}_{1 / 2}$ and $\mathcal{A}_{3 / 2}$ Amplitudes $\ldots \ldots \ldots \ldots \ldots$

5.5. Mass Dependence of Amplitudes . . . . . . . . . . 112

5.6. Ratios . . . . . . . . . . . . . . . . 119

5.7. Lattice Vacuum Saturation _ . . . . . . . . . . . 127

Chapter 6. Conclusion . . . . . . . . . . . . . . . 131 Bibliography

Appendix I. Tabulated Results and Graphs: $\beta=6.0 \ldots \ldots \ldots 138$

I.1. Pion Masses and $Z$ Values $\ldots \ldots \ldots \ldots \ldots \ldots$

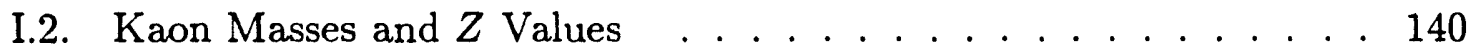

I.3. D Masses and $Z$ Values $\ldots \ldots \ldots \ldots \ldots$ 
I.4. Scalar Masses and Z Values . . . . . . . . . . . . . . 144

I.5. $\delta m$ Mass Differences . . . . . . . . . . . . . . . 146

I.6. $\mathcal{A}\left(\mathrm{f}_{\mathrm{ig}} 8 \pi\right) \ldots \ldots . . \ldots \ldots 148$

I.7. $\mathcal{A}(\mathrm{fig} 8 \mathrm{~K}) \ldots \ldots \ldots \ldots$

I.8. $\mathcal{A}($ fig8an $) \ldots \ldots \ldots$. . . . . . . . . . . . 152

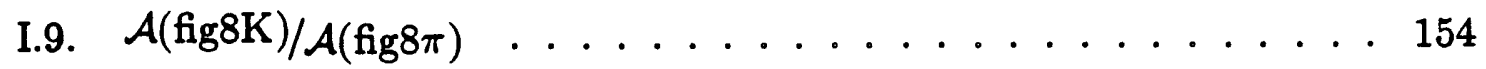

I.10. $\mathcal{A}\left(\mathrm{fig} 8 \mathrm{~K}^{V S}\right) / \mathcal{A}\left(\mathrm{fig} 8 \pi^{V S}\right) \ldots \ldots \ldots \ldots$

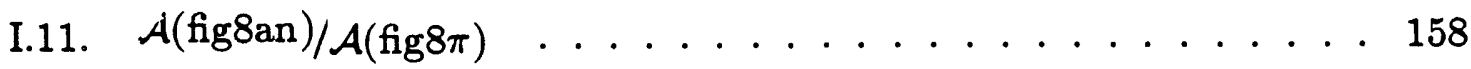

I.12. $\mathcal{A}\left(\right.$ fig8an $\left.^{V S}\right) / \mathcal{A}\left(\mathrm{fig} 8 \pi^{V S}\right) \ldots \ldots \ldots$. . . . . . . . . 160

I.13. $\mathcal{A}_{3 / 2} \ldots \ldots \ldots \ldots$

I.14. $\mathcal{A}_{3 / 2}^{V S} \ldots \ldots \ldots \ldots \ldots \ldots$

I.15. $\mathcal{A}_{3 / 2 / \mathcal{A}_{3 / 2}^{V S} \ldots \ldots \ldots \ldots} \ldots \ldots \ldots \ldots$

I.16. $\mathcal{A}_{1 / 2} \ldots \ldots \ldots \ldots$. . . . . . . . . . . . . . 168

I.17. $\mathcal{A}_{1 / 2}^{V S} \ldots \ldots \ldots \ldots \ldots \ldots \ldots$

I.18. $\mathcal{A}_{1 / 2 / \mathcal{A}_{1 / 2}^{V S} \ldots \ldots \ldots \ldots \ldots} \ldots \ldots \ldots$

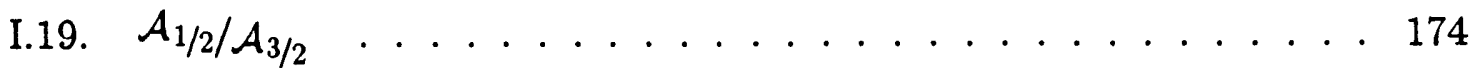

I.20. $\mathcal{A}_{1 / 2}^{V S} / \mathcal{A}_{3 / 2}^{V S} \ldots \ldots \ldots \ldots \ldots \ldots$

I.21. $\mathcal{A}$ (fig8scalar)/ $\mathcal{A}_{3 / 2} \ldots \ldots \ldots$. . . . . . . . . . . 178

1.22. Effective $1 / \mathrm{N} \ldots \ldots \ldots \ldots$ 
Appendix II. Numerical Infrastructure . . . . . . . . . . . . 182

II.1. Data Ordering . . . . . . . . . . . . . . . 182

II.2. Gauge Configurations . . . . . . . . . . . . . . . 184

II.3. Configuration Generation . . . . . . . . . . . . . . . 186

II.4. Quark Propagators . . . . . . . . . . . . . 188

II.5. Propagator Extrapolation . . . . . . . . . . . . . . 192

II.6. Matrix Inversion Algorithms . . . . . . . . . . . . . . 193

II.7. Hyperplane LU Improvement . . . . . . . . . . . . . . . . . 196

II.8. Multitasking And Hyperplane Ordering . . . . . . . . . . . 200 


\section{List of Figures}

Figure 2.3.1: Graph of $a^{-1}$ vs $\beta$. . . . . . . . . . . . . . . . . 12

Figure 3.2.1: $1+\operatorname{order}\left(g^{2}\right)$ contributions to $\mathrm{c} \rightarrow \mathrm{su} \overline{\mathrm{d}}$. . . . . . . . $2 \hat{A}$

Figure 3.3.1: Diagrams that lead lower dimension operators . . . . . 32

Figure 4.5.1: Graphical Representation of fig8 Functions . . . . . . . 59

Figure 4.5.2: $D^{0} \rightarrow \kappa$ lattice diagrams . . . . . . . . . . . . . . . 67

Figure 4.7.1: Lattice diagram for the $\pi^{+}$propagator . . . . . . . . . 72

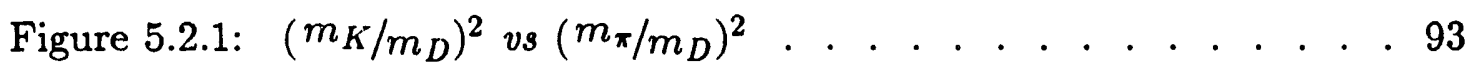

Figure 5.2.2: $m_{\text {scalar } / m_{D} v s} m_{\pi} / m_{D}$. . . . . . . . . . . . . . . . 94

Figure 5.2.3: $\delta m / m_{D} v s m_{\pi / m_{D}}$. . . . . . . . . . . . . . . . . . 96

Figure 5.3.1: TI ratio for fig $8 \pi_{1}$ and fig $8 \pi_{2}$ vs $t_{K}$. . . . . . . . . . . 99

Figure 5.3.2: TI ratio for fig $8 \mathrm{~K}_{1}$ and fig $8 \mathrm{~K}_{2}$ vs $t_{K}$. . . . . . . . . 100

Figure 5.3.3: TI ratio for fig8an ${ }_{1}$ and fig8an ${ }_{2}$ vs $t_{K}$. . . . . . . . . . 101

Figure 5.3.4: $\mathcal{A}_{1 / 2} / \mathcal{A}_{3 / 2}$ vs $t_{K}$ fit 1 . . . . . . . . . . . . . . . . 108

Figure 5.3.5: $\mathcal{A}_{1 / 2} / \mathcal{A}_{3 / 2}$ vs $t_{\underline{K}}$ fit 2 . . . . . . . . . . . . . . . . . 109

Figure 5.4.1: $\mathcal{A}_{3 / 2}$ vs $m_{\pi / m_{D}}$. . . . . . . . . . . . . . . . . . 110

Figure 5.4.2: $\mathcal{A}_{1 / 2}$ vs $m_{\pi / m_{D}}$. . . . . . . . . . . . . . . . . . 111

Figure 5.5.1: $\mathcal{A}(\mathrm{fig} 8 \pi)$ vs $m_{\pi / m_{D}}$. . . . . . . . . . . . . . . . 113

Figure 5.5.2: $\mathcal{A}\left(\right.$ fig8K) $v s m_{\pi / m_{D}}$. . . . . . . . . . . . . . . . 114

Figure 5.5.3: $\mathcal{A}\left(\right.$ fig8an) $v s m_{\pi / m_{D}}$. . . . . . . . . . . . . . . 115

Figure 5.5.4: $\mathcal{A}($ fig $8 \pi)$ divided by $m_{\pi}\left(m_{D}+m_{K}\right)$ vs $m_{\pi / m_{D}}$. . . . 118

Figure 5.6.1: $\mathcal{A}_{1 / 2} / \mathcal{A}_{3 / 2}$ vs $m_{\pi / m_{D}}$. . . . . . . . . . . . . . . 120

Figure 5.6.2: $\mathcal{A}(\mathrm{fig} 8 \mathrm{~K}) / \mathcal{A}(\mathrm{fig} 8 \pi)$ vs $m_{\pi / m_{D}}$. . . . . . . . . . . . . . . 121

Figure 5.6.3: $\mathcal{A}($ fig8an $) / \mathcal{A}($ fig8 8$)$ vs $m_{\pi / m_{D}}$. . . . . . . . . . . . . 122

Figure 5.6.4: $S U(3)_{f}$ limit $\mathcal{A}_{1 / 2} / \mathcal{A}_{3 / 2}$ vs $m_{\pi / m_{D}}$. . . . . . . . . . 124 
Figure 5.6.5: $S U(3)_{f}$ limit $\mathcal{A}(\mathrm{fig} 8 \mathrm{~K}) / \mathcal{A}(\mathrm{fig} 8 \pi)$ vs $m_{\pi / m_{D}}$. . . . . . 125

Figure 5.6.6: $\mathcal{A}($ fig8an $) / \mathcal{A}\left(\right.$ fig8 8 ) vs $m_{\pi / m_{D}}$. . . . . . . . . . . . . . . 126

Figure 5.7.1: TI ratio for fig8 $8 \pi_{1}$ and fig8 $8 \pi_{1}^{V S}$ vs $t_{K} \quad$. . . . . . . . . . 129

Figure 5.7.2: TI ratio for fig $8 \pi_{2}$ and fig $8 \pi_{2}^{V S}$ vs $t_{K}$. . . . . . . . . 130

Figure I.1.1: Pion propagator vs $t$. . . . . . . . . . . . . . . . 140

Figure I.2.1: Kaon propagator vs $t$. . . . . . . . . . . . . . . 142

Figure I.3.1: D propagator vs $t$. . . . . . . . . . . . . . . . . 144

Figure I.4.1: Scalar propagator vs $t$. . . . . . . . . . . . . . . 146

Figure I.5.1: Fit to propagator ratio vs $t$. . . . . . . . . . . . . 148

Figure I.6.1: TI ratio fig8 $8 \pi^{c o n t}$ vs $t_{K}$. . . . . . . . . . . . . . . 150

Figure I.7.1: TI ratio fig8K cont $v s t_{K} \quad$. . . . . . . . . . . . . . . . . 152

Figure I.8.1: fig8an ${ }^{\text {cont }}$ vs $t_{K}$. . . . . . . . . . . . . . . . . . 154

Figure I.9.1: Fit to fig8K/fig8 8 v $t_{K}$. . . . . . . . . . . . . . 156

Figure I.10.1: Fit to fig $8 \mathrm{~K}^{V S} / \mathrm{fig} 8 \pi^{V S}$ vs $t_{K}$. . . . . . . . . . . 158

Figure I.11.1: $\mathrm{sit}$ to fig8an/fig8 $v$ s $t_{K}$. . . . . . . . . . . . . . 160

Figure I.12.1: fig8an $^{V S} / \mathrm{FePi}^{V S}$ vs $t_{K}$. . . . . . . . . . . . . . 162

Figure I.13.1: TI ratio $G_{3 / 2}^{(4)}$ vs $t_{K}$. . . . . . . . . . . . . . . . . . 164

Figure I.14.1: TI ratio $G_{3 / 2}^{V S}$ vs $t_{K}$. . . . . . . . . . . . . . . . . . . . 166

Figure 1.15.1: Fit to $G_{3 / 2}^{(4)} / G_{3 / 2} V S$ vs $t_{K}$. . . . . . . . . . . . . . . . . . . 168

Figure I.16.1: $\quad$ TI ratio $G_{1 / 2}^{(4)}$ vs $t_{K}$. . . . . . . . . . . . . . . . . . . . . . 170

Figure I.17.1: TI ratio $G_{1 / 2}^{V S}$ vs $t_{K}$. . . . . . . . . . . . . . . . . . . . 172

Figure I.18.1: $G_{1 / 2}^{(4)} / G_{1 / 2}^{V S}$ vs $t_{K} \quad$. . . . . . . . . . . . . . . . . . . 174

Figure I.19.1: Fit to $G_{1 / 2}^{(4)} / G_{3 / 2}^{(4)}$ vs $t_{K}$. . . . . . . . . . . . . . . . . . . . . 176

Figure I.20.1: Fit to $G_{1 / 2}^{V S} / G_{3 / 2}^{V S}$ vs $t_{K}$. . . . . . . . . . . . . . . . . . . . 178

Figure I.21.1: TI ratio fig8scalar $/ G_{3 / 2}^{(4)}$ vs $t_{K} \quad$. . . . . . . . . . . . . 180 
Figure I.22.1: Fit to effective $1 / \mathrm{N} v s t_{K}$. . . . . . . . . . . . . . 182 


\section{List of Tables}

Table 3.2.1: Continuum Wilson Coefficients as a function of energy scale $\mu 27$

Table 3.3.1: Sensitivity of Lattice Wilson Coefficients to $a^{-1}$ and $\mu$. . 33

Table 4.5.1: Leading order large $\mathrm{N}$ behavior of fig8 diagrams. . . . . . 62

Table 5.1.1: Properties of available lattices . . . . . . . . . . . . . 86

Table 5.1.2: Hopping constants used . . . . . . . . . . . . . . . . 88

Table I.1.1: Pion two-point function fits . . . . . . . . . . . . . 139

Table I.2.1: Kaon two- roint function fits . . . . . . . . . . . . . 141

Table I.3.1: D two-point function fits . . . . . . . . . . . . . . 143

Table I.4.1: Scalar two-point function fits . . . . . . . . . . . . 145

Table I.5.1: Fits to propagator ratio vs $t$. . . . . . . . . . . . . 147

Table I.6.1: TI ratio fig8 $8 \pi^{\text {cont }}$ vs $t_{K}$. . . . . . . . . . . . . . . . . 149

Table I.7.1: TI ratio fig8K $\mathrm{K}^{\text {cont }}$ vs $t_{K}$. . . . . . . . . . . . . . . 151

Table I.8.1: TI ratio fig8an ${ }^{\text {cont }}$ vs $t_{K}$. . . . . . . . . . . . . . . 153

Table I.9.1: Fits to fig8K/fig8 $v s t_{K}$. . . . . . . . . . . . . . 155

Table I.10.1: Fits to fig8K $\mathrm{K}^{V S} / \mathrm{fig} 8 \pi^{V S}$ vs $t_{K}$. . . . . . . . . . . 157

Table I.11.1: fig8an/fig8n vs $t_{K}$. . . . . . . . . . . . . . . . . . . . . 159

Table I.12.1: fig8an $V S /$ fig8 $8 S$ vs $t_{K} \quad$. . . . . . . . . . . . . . . . 161

Table I.13.1: TI ratio $G_{3 / 2}^{(4)}$ vs $t_{K} \quad$. . . . . . . . . . . . . . . . . . . . . . 163

Table I.14.1: TI ratio $G_{3 / 2}^{V S}$ vs $t_{K} \quad$. . . . . . . . . . . . . . . . . . . . 165

Table I.15.1: Fits to $G_{3 / 2}^{(4)} / G_{3 / 2}$ VS $v s t_{K}$. . . . . . . . . . . . . . . . . . . . 167

Table I.16.1: TI ratio $G_{1 / 2}^{(4)}$ vs $t_{K} \quad$. . . . . . . . . . . . . . . . . . . . . . . . . . 169

Table I.17.1: TI ratio $G_{1 / 2}{ }^{V S}$ vs $t_{K} \quad$. . . . . . . . . . . . . . . . . . . . 171

Table I.18.1: Fits to $G_{1 / 2}^{(4)} / G_{1 / 2}$ VS $v s t_{K}$. . . . . . . . . . . . . . . . . . . 173

Table I.19.1: Fit to $G_{1 / 2}^{(4)} / G_{3 / 2}^{(4)}$ vs $t_{K}$. . . . . . . . . . . . . . . . . . . 175 
Table I.20.1: Fits to $G_{1 / 2}^{V S} / G_{3 / 2}^{V S}$ vs $t_{K} \quad$. . . . . . . . . . . . . . . . . . 177

Table I.21.1: $\quad$ TI ratio fig8scalar $/ G_{3 / 2}^{(4)}$ vs $t_{K} \quad$. . . . . . . . . . . . . . . . . 179

Table I.22.1: Fits to effective $1 / \mathrm{N} v s t_{K}$. . . . . . . . . . . . . . 181 


\section{ACKNOWLEDGMENTS}

I wish to thank Professors Amarjit Soni and Claude Bernard for their invaluable guidance and encouragement during the course of this research. As part of a continuing project in weak matrix element calculations, this work has its basis in the collaborative efforts of many. In particular, I thank and acknowledge the efforts of the members of this group with whom I have the pleasure of working: Aida El-Khadra, Jim Labrenz, and Kenton Yee. I thank the high energy theory group at Brookhaven National Laboratory for their support over the past two years. I am grateful for the assistance, encouragement and friendship of the individual members of the Brookhaven theory group. Their expertise and enthusiasm combine to create a stimulating atmosphere in which to do research. Thanks go to Tanmoy Bhattacharya, Arvind Borde and Frank Paige for lending their TEXnical expertise. My special and heartfelt thanks go to Geor sia Schwender for being exceptionally supportive during the writing of this dissertation. It is truly impossible to thank her for all the encouragement and help she provided in so many ways. 


\title{
ABSTRACT OF THE DISSERTATION
}

\section{Lattice Calculation of Nonleptonic Charm Decays}

\author{
by \\ James Nicholas Simone \\ Doctor of Philosophy in Physics \\ University of California, Los Angeles, 1991 \\ Professor Claude Bernard Co-chair \\ Professor Amarjit Soni Co-chair \\ Professor Roberto Peccei, ex officio Chair
}

The decays of charmed mesons into two body nonleptonic final states are investigated. Weak interaction amplitudes of interest in these decays are extracted from lattice four-point correlation functions using an effective weak Hamiltonian including effects to order $G_{f}$ in the weak interactions yet containing effects to all orders in the strong interactions.

The lattice calculation allows a quantitative examination of nonspectator processes in charm decays helping to elucidate the role of effects such as color coherence, final state interactions and the importance of the so called weak annihilation process. For $D \rightarrow K \pi$, we find that the non-spectator weak annihilation diagram is not small, and we interpret this as evidence for large final state interactions. Moreover, 
there is indications of a resonance in the isospin $1 / 2$ channel to which the weak annihilation process contributes exclusively.

Findings from the lattice calculation are compared to expectations of continuum methodology. Amplitudes are compared to results from the continuum vacuum saturation approximation and amplitudes are examined within the framework of the $1 / \mathrm{N}$ expansion.

Factorization and the vacuum saturation approximation are tested for lattice amplitudes by comparing amplitudes extracted from lattice four-point functions with the same amplitude extracted from products of two-point and three-point lattice correlation functions arising out of factorization and vacuum saturation. 


\section{Chapter 1. \\ Introduction}

The development of a theoretical understanding of charm decays began in the 1970 's with the the spectator model ${ }^{1-2}$.

The inadequacy of this model quickly became apparent once sufficient data on $D$ decays became available. For example, the ratio of $D^{+}$to $D^{0}$ lifetimes is ${ }^{3}$

$$
\frac{\tau\left(D^{+}\right)}{\tau\left(D^{0}\right)}=2.5 \pm .1
$$

whereas this ratio is predicted to be approximately one in the spectator model. The failure of the simple spectator model for the $D$ system focused the attention of the physics community upon the importance of QCD effects in $D$ decays.

Steady progress has been made in understanding both theoretical and phenomenological aspects of charm decays ${ }^{4-10}$. Despice this progress, a clear understanding of charm decays is still hampered by the difficulty of performing the necessary nonperturbative calculations.

Meanwhile, an impressive body of experimental data accumulated over the past decade ${ }^{11}$ allows ever more precise tests of theoretical models.

In this exploratory study we propose to employ charm decays as a testing ground where we hope to develop the necessary lattice techniques to allow a first principles calculation of the required matrix elements. Such a calculation would serve as a crucial check of our theoretical understanding of charm decays. An understanding of the underlying nonperturbative QCD effects in charm decays, although valuable in itself, also forms the first step in understanding similar effects in $\mathrm{B}$ decays. There such effects are expected to have important repercussions for CP violation searches ${ }^{12-13}$. 
Semileptonic decay rates indicate the importance of studying ronleptonic decays. The inclusive semileptonic rates for $D^{+}$and $D^{0}$ are predicted to be approximately equal

$$
\Gamma\left(D^{+} \rightarrow e^{+} X\right)=\Gamma\left(D^{0} \rightarrow e^{+} X\right)+\operatorname{order}\left(\sin ^{2} \theta_{c}\right)
$$

which follows from isospin invariance and the Cabibbo allowed decay process:

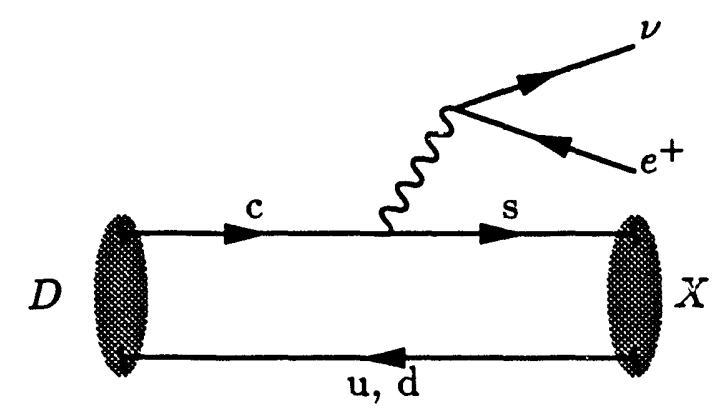

However, experimental measurements ${ }^{3}$ of the inclusive branching fractions yield

$$
\begin{aligned}
& \text { b.f. }\left(D^{+} \rightarrow e^{+} X\right)=19.2^{+1.7} \% \\
& \text { b.f. }\left(D^{0} \rightarrow e^{+} X\right)=7.7 \pm 1.2 \%
\end{aligned}
$$

The difference in the branching fractions directs attention to the nonleptonic channels.

Current lattice techniques for calculating weak matrix elements limit our study to exclusive two body final state decay modes. A study of two body decay modes should still allow us to determine the relative importance of such proposed phenomena as weak annihilation, Pauli interference, color coherence, and final state interactions in $D$ decays.

As an example, we note that experiment indicates for $D^{0} \rightarrow K^{-} \pi^{+}$and $D^{+} \rightarrow$ $\bar{K}^{0} \pi^{+}$decay amplitudes ${ }^{3}$ :

$$
\frac{\left|. \mathcal{A}\left(D^{0} \rightarrow K^{-} \pi^{+}\right)\right|}{\left|\mathcal{A}\left(D^{+} \rightarrow \bar{K}^{0} \pi^{+}\right)\right|}=1.83^{+.30}-.25
$$


At the valence quark level, one possible contribution to both the $D^{0}$ and $D^{+}$ amplitudes comes through the spectator process. The charm quark decays into a strange quark and a $W$ boson which subsequently decays into an up and down quark. The light up or down valence quark of the $D$ meson acts as a spectator during the charm decay. The final state quarks then hadronize as shown:

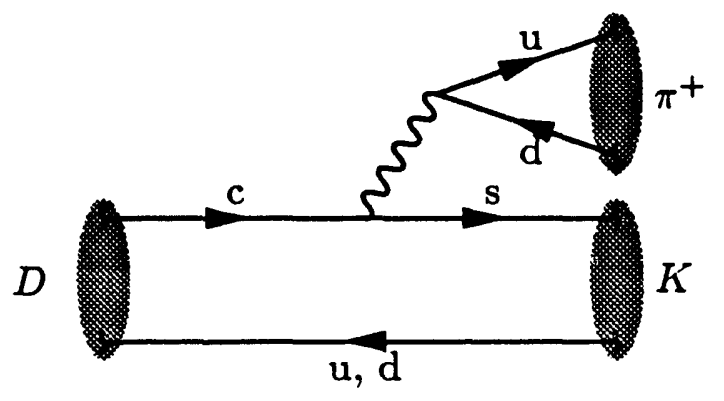

In the spectator model this process is assumed to dominate decays like those in Eq. (1.4). The experimental value of this ratio indicates that processes neglected in the spectator model are important. There are additional contributions at the Cabibbo allowed level to each of the channels considered in Eq. (1.4). The total amplitude for each channel will then be the sum of the spectator amplitude plus the amplitude(s) for any additional process(es).

For the $D^{+} \rightarrow \vec{K}^{0} \pi^{+}$channel there is a second spectator-type process in which the two down quarks in the final state of the spectator process above are interchanged:

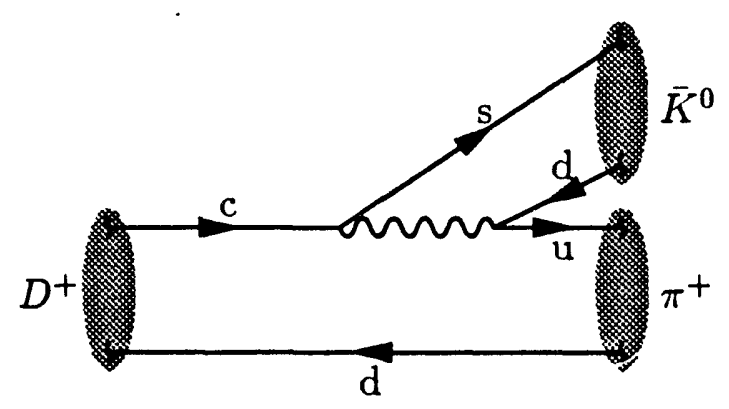

The interchange of these two fermions might result in destructive interference between this amplitude and the spectator amplitude. The amplitude for $\mathrm{D}^{+} \rightarrow$ 
$\bar{K}^{0} \pi^{+}$would then be reduced by this so called Pauli interference. Note that such a reduction in the $D^{+}$amplitude may explain why the ratic in Eq. (1.4) is greater than one $e^{9}$.

The $W$ boson does not carry color quantum numbers and the final state mesons must be color singlets. Therefore, if QCD corrections are neglected, this second "color suppressed" spectator process is reduced in magnitude by a factor of $1 / 3$ relative to the spectator process. The amplitude of the second process can then only partially cancel the spectator amplitude. With QCD corrections, the degree of color suppression will be altered due to the exchange of color between valence quarks via gluons.

On the lattice, one hopes to calculate the amplitudes of the processes analogous to the spectator and the color suppressed spectator. It would then be possible to measure the degree of color suppression and make comparisons to both the factor of $1 / 3$ found above by simple color counting and, more importantly, to more sophisticated models that attempt to include the QCD corrections.

The additional process contributing to the $D^{0} \rightarrow K^{-} \pi^{+}$channel is the nonspectator "weak annihilation" process in which the $W$ boson is exchanged between the charm and up valence quarks of the $D^{0}$ :

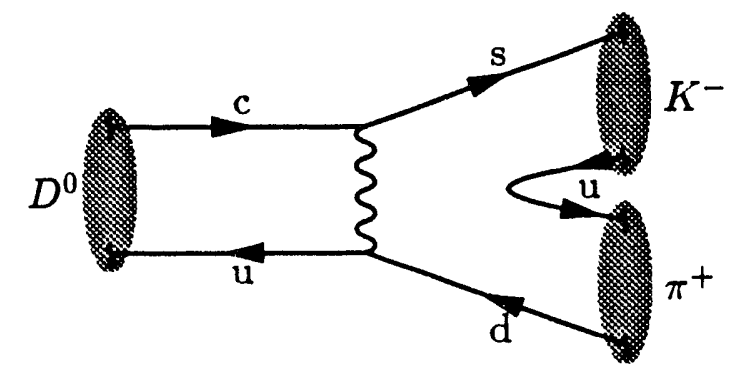

This process occurs for Cabibbo allowed $D^{0}$ decays but not for Cabibbo allowed $D^{+}$decays. If the weak annihilation amplitude adds constructively with 
the spectator process amplitude, it could potentially be part of the explanation of the observed value for the ratio in Eq. (1.4).

The role of "he "weak annihilation" process in $D$ decays is a topic of continuing theoretical interist. Note that the creation of the $u \bar{u}$ pair from the "vacuum" is likely to be due to gluon emission which indicates the crucial nature of QCD corrections in this process. These QCD corrections may effectively overcome the helicity suppression at the $W$ vertex caused by the relatively small value of the spectator mass and the vector nature of the weak current ${ }^{8}$. A large amplitude for the weak annihilation process may further suggest larger than expected resonance effects in decay channels into which the weak annihilation process can contribute.

An important goal of this study of nonleptonic decays is to examine the role of the weak annihilation process. In the lattice computation, the manifest inclusion of nonperturbative effects that may be crucial to weak annihilation, and which are otherwise difficult to estimate, is an exciting prospect.

QCD effects also appear in nonleptonic $D$ decays in the form of large final state interactions.

Consider the $D \rightarrow K \pi$ decay modes:

$$
\begin{aligned}
& D^{0} \rightarrow K^{-} \pi^{+} \\
& D^{0} \rightarrow \bar{K}^{0} \pi^{0} \\
& D^{+} \rightarrow \bar{K}^{0} \pi^{+}
\end{aligned}
$$

The amplitudes for these decays can be written in terms of $\mathcal{A}_{1 / 2}$ and $\mathcal{A}_{3 / 2}$ that are the amplitudes to decay into final states of definite isospin $1 / 2$ and $3 / 2$ respectively.

$$
\begin{aligned}
\mathcal{A}\left(D^{0} \rightarrow K^{-} \pi^{+}\right) & =\sqrt{\frac{1}{3}} \mathcal{A}_{3 / 2}+\sqrt{\frac{2}{3}} \mathcal{A}_{1 / 2} \\
\mathcal{A}\left(D^{0} \rightarrow \bar{K}^{0} \pi^{0}\right) & =\sqrt{\frac{2}{3}} \mathcal{A}_{3 / 2}-\sqrt{\frac{1}{3}} \mathcal{A}_{1 / 2} \\
\mathcal{A}\left(D^{+} \rightarrow \bar{K}^{0} \pi^{+}\right) & =\sqrt{3} \mathcal{A}_{3 / 2}
\end{aligned}
$$


These decay amplitudes then must satisfy the isospin sum rule

$$
\mathcal{A}\left(D^{0} \rightarrow K^{-} \pi^{+}\right)+\sqrt{2} \mathcal{A}\left(D^{0} \rightarrow \bar{K}^{0} \pi^{0}\right)=\mathcal{A}\left(D^{+} \rightarrow \bar{K}^{0} \pi^{+}\right)
$$

An analysis of the experimental data ${ }^{14}$ indicates these data are best fit with a relatively large nontrivial phase difference between the $\mathcal{A}_{1 / 2}$ and $\mathcal{A}_{3 / 2}$ amplitudes

$$
\delta_{1 / 2}-\delta_{3 / 2}=(77 \pm 11)^{\circ}
$$

with magnitude

$$
\frac{\left|\mathcal{A}_{1 / 2}\right|}{\left|\mathcal{A}_{3 / 2}\right|}=3.67 \pm .27
$$

where $\mathcal{A}_{1 / 2}=\left|\mathcal{A}_{1 / 2}\right| e^{\delta_{1}} / 2$ and $\mathcal{A}_{3 / 2}=\left|\mathcal{A}_{3 / 2}\right| e^{\delta_{3} / 2}$.

The amplitudes $\mathcal{A}_{1 / 2}$ and $\mathcal{A}_{3 / 2}$ calculated on the lattice are Euclidean and therefore they are relatively real. Final state interactions on the lattice then do not appear as complex phases multiplying the weak interaction matrix elements as they do in Minkowski space. Understanding how final state interactions are manifested on the lattice is crucial to the interpretation of the lattice results. 


\section{Chapter 2. \\ Lattice Fundamentals}

\subsection{Continuum Lagrangian}

This chapter is a brief introduction to Lattice Gauge Theory. It is a guide to the notational conventions used for $\mathrm{QCD}$ on the lattice. For brevity, the rigorous derivations have been kept to a minimum. Discussions of lattice gauge theories are found in several references ${ }^{15-18}$.

The QCD Lagrangian density is given by

$$
\mathcal{L}=\bar{\Psi}\left[i \not \supset-m_{0}\right] \Psi-\frac{1}{2} \operatorname{Tr}\left(G^{\mu \nu} G_{\mu \nu}\right)
$$

Matrix notation is used so that color indices are suppressed and $\Psi(x)$ represents a vector in both flavor space and color space. The covariant derivative is taken to be

$$
D_{\mu}=\partial_{\mu}+i g_{0} A_{\mu}
$$

where $g_{0}$ is the bare QCD coupling constant. The gluon field tensor then becomes

$$
G_{\mu \nu}=\partial_{\mu} A_{\nu}-\partial_{\nu} A_{\mu}+i g_{0}\left[A_{\mu}, A_{\nu}\right]
$$

The Yang-Mills fields are compactly written

$$
A_{\mu}=A_{\mu}^{a} \lambda^{a}
$$


in matrix notation where $\lambda^{a}$ represents the generators of the $S I I(3)$ color group. The generators are normalized so that

$$
\operatorname{Tr}\left(\lambda^{a} \lambda^{b}\right)=\frac{1}{2} \delta^{a b}
$$

The Lagrangian density Eq. (2.1.1) is gauge invariant under local gauge transformations $g(x) \in S U(3)$ of the form

$$
\begin{aligned}
\psi(x) & \rightarrow g(x) \psi(x) \\
\bar{\psi}(x) & \rightarrow \bar{\psi}(x) g^{-1}(x) \\
A_{\mu}(x) & \rightarrow g(x) A_{\mu}(x) g^{-1}(x)-\frac{1}{i g_{0}}\left(\partial_{\mu} g(x)\right) g^{-1}(x)
\end{aligned}
$$

\subsection{The Lattice Gauge Action}

Start with a discrete hypercubical lattice of space-time points with finite volume and Euclidean metric. The minimum spacing between space-time points on this lattice is the lattice spacing $a$.

Fourier analysis shows that a dynamical theory defined on such a lattice with periodic boundary conditions has both an infrared and an ultraviolet cutoff. On a lattice of spatial volume $\left(a n_{s}\right)^{3}$, three-momenta are discrete taking possible values

$$
\vec{p}=\frac{2 \pi}{a n_{s}}\left(n_{1}, n_{2}, n_{3}\right)
$$

where $-n_{s} / 2<n_{i} \leq n_{s} / 2$ for even $n_{s}$. The finite number of possible momenta values acts as an infrared cutoff. The individual components of the momenta are bounded by $\pi / a$. The lattice then provides a regulator for any field theory defined upon it. 
In lattice QCD we define a theory that has as its limit the theory describeci in section 2.1 when both the lattice spacing $a$ is taken to zero and the volume of the lattice $\left(a n_{s}\right)^{3}$ is taken to infinity in a prescribed way.

A fundamental symmetry of QCD is the local gauge symmetry described by Eqs. (2.1.6). An essential requirement of lattice QCD is that this local gauge symmetry be manifestly preserved. In the continuum theory the path ordered quantity

$$
\bar{\Psi}(y) \text { P.O. } \exp \left(-i g_{0} \int_{x}^{y} d^{\mu} z A_{\mu}(z)\right) \Psi(x)
$$

is gauge invariant. The gauge fields can be given the geometric interpretation defining the parallel transport of matter fields.

Recall that in general relativity the notion of parallel transport around a closed path measures the local curvature and thus the local gravitational field. Extending this concept to $\mathrm{QCD}$, consider path ordered products of color fields around a closed path.

Represent the gauge fields by link matrices $U_{\mu}(x) \in S U(3)$ defined through

$$
U_{\mu}(x)=P . O \cdot e^{i g_{0} \int_{x}^{x+\tilde{\mu}} d^{\nu} z A_{\nu}(z)} \rightarrow e^{i a g_{0} A_{\mu}(x)}
$$

where the fundamental representation of $S U(3)$ is used and where the direction vectors $\hat{\mu}$ have length $|\hat{\mu}|=a$. Under a local gauge transformation $\mathcal{G}$, the link matrices transform as

$$
\mathcal{G}: U_{\mu}(x) \rightarrow U_{\mu}^{\prime}(x)=V(x) U_{\mu}(x) V^{\dagger}(x+\hat{\mu})
$$

Consider the trace of a path ordered product $P_{\square}$ of link matrices around a plaquette (the smallest planar closed path defined on the lattice). For example, 
starting from site $x$ and going first in direction $\hat{\mu}$ and then in direction $\hat{\nu}$ and finally closing the square path

$$
P_{\square}=\operatorname{Tr}\left(U_{\mu}(x) U_{\nu}(x+\hat{\mu}) U_{\mu}^{\dagger}(x+\hat{\nu}) U_{\nu}^{\dagger}(x)\right)
$$

the product of link matrices is given the pictorial interpretation:

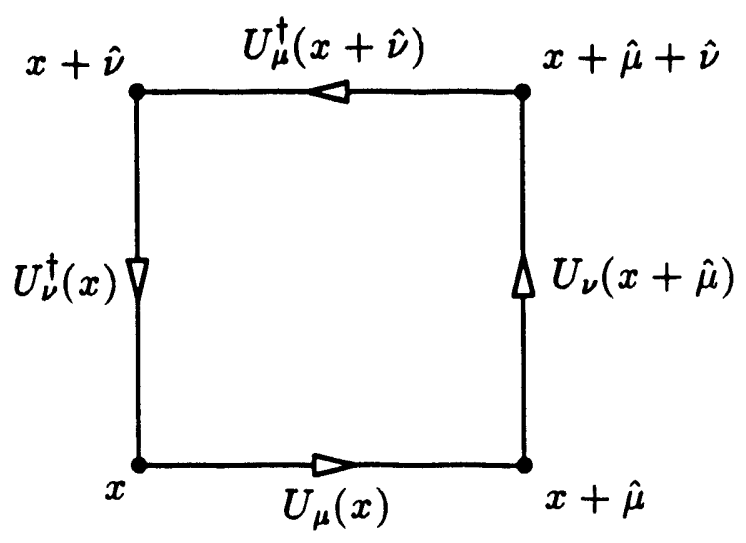

Tine lattice sites at the corners of the plaquette are labeled by their spacetime coordinates and the directed line segments represent the corresponding link matrices. The quantity $P \square$ is gauge invariant as can be verified by performing a gauge transformation as in Eq. (2.2.4).

With these definitions a possible choice for the action is the Wilson Gauge action $^{19}$

$$
S_{g}=\frac{2 N_{c}}{g_{0}^{2}} \sum_{\square}\left\{1-\frac{1}{N_{c}} \operatorname{Re} P_{\square}\right\}
$$

where the sum is over ali plaquettes and $N_{c}$ is the number of colors $\left(N_{c}=3\right.$ for QCD). This action is locally gauge invariant. In naive continuum limit $(a \rightarrow 0)$ this action contains the pure gauge term of Eq. (2.1.1). The $\beta$ value is defined by

$$
\beta \equiv \frac{2 N_{c}}{g_{0}^{2}}
$$

for $S U\left(N_{c}\right)$. 


\subsection{The Continuum Limit}

Consider a dimensionful quantity such as the mass $m$ of the lowest lying excitation of this theory. Define the correlation length $\xi$ measured in lattice units

$$
\xi=\frac{1}{a m}
$$

As we approach the continuum limit $a \rightarrow 0$ suppose the mass $m$ approaches a finite nonzero physical value. The correlation length must then diverge. In the language of statistical mechanics this behavior is indicative of a second order phase transition. A continuum field theory can then be recovered from the discrete lattice field theory at such a second order phase transition.

Renormalization group studies relate the lattice spacing to the value of $\beta$ for $\beta \gg 0$

$$
a=\frac{1}{\Lambda^{l a t}}\left(\frac{N_{c} b_{0}}{8 \pi^{2} \beta}\right)^{-\frac{b_{1}}{2 b_{0}^{2}}} \exp \left(-\frac{4 \pi^{2}}{N_{c} b_{0}^{2}} \beta\right)\left[1+o\left(\frac{1}{\beta}\right)\right]
$$

As $\beta \rightarrow \infty$ the lattice spacing goes to zero. Then, the behavior of the correlation length

$$
\xi^{-1} \sim e^{-1 / g_{0}^{2}}
$$

indicates an essential singularity at $g_{0}=0$ and thus the necessary second order behavior to define a continuum limit.

Numerical simulations must of necessity be performed at finite lattice spacing. For $\beta$ near enough to the critical point the scaling relation Eq. (2.3.1) is used to set the scale $\Lambda^{\text {latt }}$. Measured quantities on the lattice are dimensionless since they are measured in lattice units. Thus, for the string tension parameter $\sigma$ in the infinitely heavy quark-quark potential one numerically determines $a^{2} \sigma$ on the lattice. Studying the behavior of this quantity as a function of $\beta$ according to 


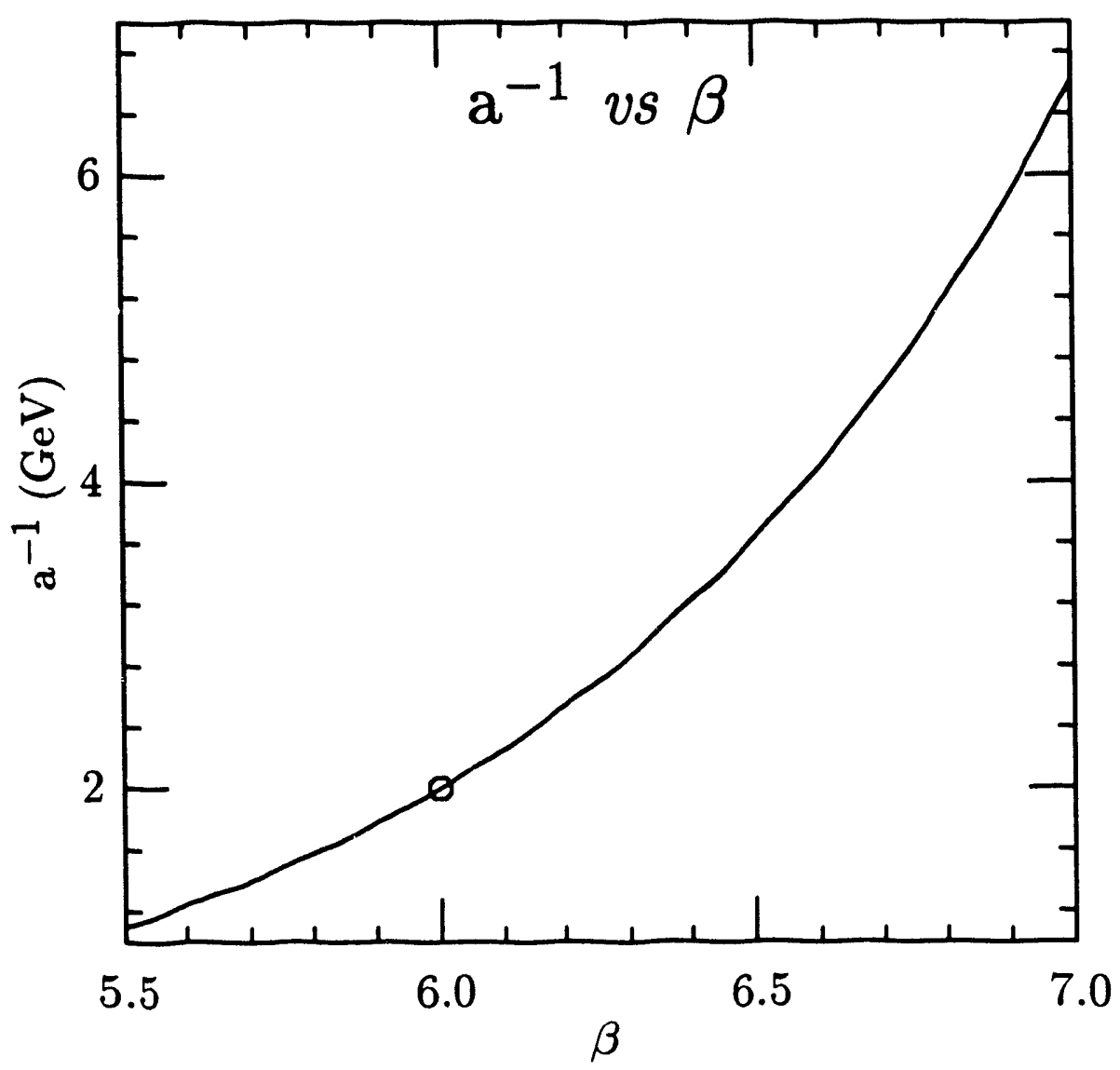

Figure 2.3.1: Graph of $a^{-1}$ as a function of $\beta$ obtained from the scaling relation Eq. (2.3.1). The values shown assume that the scaling relation is valid over the region shown and a value of $a^{-1}=2.0 \mathrm{GeV}$ at $\beta=6.0$ (marked point) as estimated from string tension measurements.

Eq. (2.3.1) allows the quantity $\sigma / \Lambda_{\text {lat }}^{2}$ to be numerically determined within the "scaling window" for a fixed lattice size. From this ratio and the dimensionful value of the string tension derived from experiment, the lattice spacing as a function of $\beta$ and $\Lambda^{\text {lat }}$ can be set.

Figure 2.3.1 shows the expected scaling behavior of the inverse lattice spacing $a^{-1}$ versus $\beta$. The scale was set by the nominal value $a^{-1}=2.0 \mathrm{GeV}$ at $\beta=6.0$. This value is consistent with string tension measurements. 


\subsection{Euclidean Dirac Matrices}

Minkowski space Dirac matrices (Bjorken and Drell ${ }^{20}$ convention) are transformed to the Euclidean space matrices as follows:

$$
\gamma^{0} \rightarrow \gamma^{4} \quad \gamma^{j} \rightarrow i \gamma^{j} \quad \gamma^{5} \rightarrow \gamma^{5}
$$

The Euclidean gamma matrices then have the properties:

$$
\gamma^{\mu \dagger}=\gamma^{\mu} \quad\left\{\gamma^{\mu}, \gamma^{\nu}\right\}=2 \delta^{\mu \nu}
$$

\subsection{Wilson Fermion Action}

The lattice fermion action can be written as a bilinear in the fermion field variables $\bar{\Psi}$ and $\Psi$

$$
S_{f}=\sum_{x, y} \bar{\Psi}(x) \mathrm{Q}^{-1}(x, y ; \mathcal{U}) \Psi(y)
$$

where the sums over $x$ and $y$ represent not only sums over the coordinates but spin and color degrees of freedom as well. The quantity $Q^{-1}$ is the inverse quark propagator.

Discretizing the fermion action in Eq. (2.1.1) by replacing the covariant derivative with the finite difference

$$
D_{\mu} \Psi(x) \rightarrow \frac{1}{2 a}\left[U_{\mu}(x) \Psi(x+\hat{\mu})-U_{\mu}^{\dagger}(x-\hat{\mu}) \Psi(x-\hat{\mu})\right]
$$

leads to the naive fermion action. The naive fermion action suffers from the so called fermion doubling problem. In $d$ spacetime dimensions, $2^{d}$ degenerate 
fermion species appear. Pairs of opposite chirality appear so as to exactly cancel the axial anomaly ${ }^{21}$.

The mass degeneracy among naive fermion species may be lifted by adding an irrelevant operator (an operator that vanishes in the continuum limit) to the naive action. The Wilson action ${ }^{22}$ results from the addition of a second difference term

$$
\Delta \mathcal{L}=-\frac{r}{2 a} \sum_{\mu} \bar{\Psi}(x)\left[U_{\mu}(x) \Psi(x+\hat{\mu})-2 \Psi(x)+U_{\mu}^{\dagger}(x-\hat{\mu}) \Psi(x-\hat{\mu})\right]
$$

to the discrete version of Eq. (2.1.1). The arbitrary parameter $r$ is set to one in the work reported in this dissertation.

The inverse quark propagator then becomes

$$
\mathrm{Q}^{-1}(x, y ; \mathcal{U})=\delta_{x, y}-\kappa \sum_{\mu=1}^{4}\left\{\left(r-\gamma^{\mu}\right) U_{\mu}(x) \delta_{x+\hat{\mu}, y}+\left(r+\gamma^{\mu}\right) U_{\mu}^{\dagger}(y) \delta_{x-\hat{\mu}, y}\right\}
$$

for Wilson fermions. The hopping constant $\kappa$ is related to the bare quark mass by

$$
\kappa \equiv \frac{1}{2 a m_{0}+8 r}
$$

The fermi fields in the expression above are made dimensionless by the rescaling

$$
\psi^{\text {cont }}=\left(\frac{2 \kappa}{a^{3}}\right)^{\frac{1}{2}} \psi^{\text {lattice }}
$$

in order to simplify the lattice expression.

For completeness, note that from the form of the gauge invariant bilinear

$$
\bar{\Psi}(x+\hat{\mu}) U_{\mu}^{\dagger}(x) \Psi(x)
$$

the matter fields transform as follows under the gauge transformation in Eq. (2.2.4)

$$
\mathcal{G}: \Psi(x) \rightarrow \Psi^{\prime}(x)=V(x) \Psi(x)
$$


and

$$
\mathcal{G}: \bar{\Psi}(x) \rightarrow \bar{\Psi}^{\prime}(x)=\bar{\Psi}(x) V^{\dagger}(x)
$$

\subsection{Discrete Symmetries of Quark Propagators}

Quark propagators on the lattice can be shown to satisfy several identities based on their transformation properties under the discrete symmetries $\mathrm{C}$ (charge conjugation), P (parity), T (euclidean time reversal), and Hermiticity ${ }^{23}$.

These properties of the quark propagators are used to relate correlation functions in a fixed background gauge field to the correlation function in a fixed tackground field related to the original gauge field by one of the symmetries above.

\subsubsection{Hermiticity}

The identity

$$
\mathrm{Q}^{-1}(x, y ; \mathcal{U})=\gamma^{5} \mathrm{Q}^{-1^{\dagger}}(y, x ; \mathcal{U}) \gamma^{5}
$$

is shown to hold for the inverse quark propagator on the lattice by comparison of the explicit expressions following from Eq. (2.5.4) for the left and right hand sides of the identity. The quark propagator satisfies

$$
\sum_{z} \mathrm{Q}(\ddot{u}, z ; \mathcal{U}) \mathrm{Q}^{-1}(z, y ; \mathcal{U})=\delta_{x, y}
$$

where in this notation the sum over coordinates $z$ includes summation over repeated (suppressed) spin and color indices. Take the Hermitian conjugate of Eq. (2.6.2). Multiply on the left and right by $\gamma^{5}$, insert a factor of $\gamma^{5} \gamma^{5}=1$ then use Eq. (2.6.1) to find

$$
\sum_{z} \mathrm{Q}^{-1}(y, z ; \mathcal{U}) \gamma^{5} \mathrm{Q}^{\dagger}(x, z ; \mathcal{U}) \gamma^{5}=\delta_{y, x}
$$


Then, it follows that

$$
\mathrm{Q}(z, x ; \mathcal{U})=\gamma^{5} \mathrm{Q}^{\dagger}(x, z ; \mathcal{U}) \gamma^{5}
$$

which is the identity obeyed by the quark propagator. Hermiticity relates the quark propagator from $x$ to $z$ to the quark propagator from $z$ to $x$.

\subsubsection{Charge Conjugation}

Define the charge conjugation matrix $C=\gamma^{4} \gamma^{2}$. Matter and gauge fields have the transformation properties

$$
\begin{aligned}
\mathcal{C}: \quad \psi(x) & \rightarrow C \bar{\psi}^{T}(x) \\
\bar{\psi}(x) & \rightarrow-\psi^{T}(x) C^{-1} \\
U_{\mu}(x) & \rightarrow U_{\mu}^{*}(x)
\end{aligned}
$$

under charge conjugation. Take the charge conjugated grüge variables to be $U_{\mu}^{C}=U_{\mu}^{*}$. Then, the inverse quark propagator satisfies the identity

$$
\mathrm{Q}^{-1}(x, y ; \mathcal{U})=C \mathrm{Q}^{-1}\left(y, x ; \mathcal{U}^{C}\right) C^{-1}
$$

which can be verified given the explicit expression Eq. (2.5.4) for the inverse quark propagator $Q^{-1}$. It then follows from Eq. (2.6.2) that

$$
\mathrm{Q}(x, y ; \mathcal{U})=C \mathrm{Q}^{T}\left(y, x ; \mathcal{U}^{C}\right) C^{-1}
$$

is an identity for quark propagators. 


\subsubsection{Parity}

The transformation rules of the fields under parity are:

$$
\begin{aligned}
\mathcal{P}: \quad \psi(x) & \rightarrow \gamma^{4} \psi\left(x^{P}\right) \\
\bar{\psi}(x) & \rightarrow \bar{\psi}\left(x^{P}\right) \gamma^{4} \\
U_{j}(x) & \rightarrow U_{j}^{\dagger}\left(x^{P}-\hat{\jmath}\right) \\
U_{4}(x) & \rightarrow U_{4}\left(x^{P}\right)
\end{aligned}
$$

Where $x^{P}=(t,-\vec{x})$ for $x=(t, \vec{x})$. Take $\mathcal{U}^{P}$ to be the configuration found by parity transforming the individual gauge variables as described above. Then,

$$
\mathrm{Q}^{-1}(x, y ; \mathcal{U})=\gamma^{4} \mathrm{Q}^{-1}\left(x^{P}, y^{P} ; \mathcal{U}^{P}\right) \gamma^{4}
$$

and again Eq. (2.6.2) is used to show

$$
\mathrm{Q}(x, y ; \mathcal{U})=\gamma^{4} \mathrm{Q}\left(x^{P}, y^{P} ; \mathcal{U}^{P}\right) \gamma^{4}
$$

for quark propagators.

\subsubsection{Time Inversion}

Define the matrix $T=\gamma^{4} \gamma^{5}$. Then, for Euclidean time inversion

$$
\begin{aligned}
\mathcal{T}: \quad \psi(x) & \rightarrow T \psi\left(x^{T}\right) \\
\bar{\psi}(x) & \rightarrow \psi\left(x^{T}\right) T^{-1} \\
U_{j}(x) & \rightarrow U_{j}\left(x^{T}\right) \\
U_{4}(x) & \rightarrow U_{4}^{\dagger}\left(x^{T}-\hat{\mu}\right)
\end{aligned}
$$


where $x^{T}=(-t, \vec{x})$. Define $\mathcal{U}^{T}$ to be the configuration resulting from the application of the transformation rules above for link gauge variables. Then, by similar arguments to those used in the previous sections, the identity

$$
\mathrm{Q}(x, y ; \mathcal{U})=T \mathrm{Q}\left(x^{T}, y^{T} ; \mathcal{U}^{T}\right) T^{-1}
$$

is found to hold for the quark propagators.

\subsection{Expectation Values}

The Euclidean generating functional of quantum field theory is

$$
Z[\bar{\eta}, \eta]=\frac{1}{\mathcal{N}} \int \mathcal{D} \bar{\psi} \mathcal{D} \psi \mathcal{D} U e^{-S_{g}-S_{f}+\bar{\eta} \Psi+\Psi \eta}
$$

where $\mathcal{N}$ is a normalization factor and $\bar{\eta}$ and $\eta$ are anticommuting c-number sources. The quantity $Z[0,0]$ has a clear analogy to the partition function of statistical mechanics. In the quantum theory the exponential Boltzmann weight factor arises from the quantum mechanical action. This analogy to a thermal system allows Monte Carlo methods to be exploited in numerical lattice QCD calculations.

The form of the fermion action Eq. (2.5.1) allows the integrations over the fermion degrees of freedom to be performed with the result

$$
Z[\bar{\eta}, \eta]=\frac{1}{\mathcal{N}} \int \mathcal{D} U \operatorname{det}\left(\mathrm{Q}^{-1}(\mathcal{U})\right) e^{\bar{\eta} Q \eta} e^{-S_{g}}
$$

Fermionic operator $\mathcal{O}\left(\bar{\psi}\left(x_{1}\right), \ldots, \bar{\psi}\left(x_{n}\right), \psi\left(y_{1}\right), \ldots, \psi\left(y_{n}\right)\right)$ has the expectation value $\langle 0|\mathcal{O}| 0\rangle$ formally defined by

$$
\begin{aligned}
\langle 0|\mathcal{O}| 0\rangle & =\left.\mathcal{O}\left(\frac{\delta}{\delta \eta\left(x_{1}\right)}, \ldots, \frac{\delta}{\delta \eta\left(x_{n}\right)}, \frac{\delta}{\delta \bar{\eta}\left(y_{1}\right)}, \ldots, \frac{\delta}{\delta \bar{\eta}\left(y_{n}\right)}\right) e^{W[\bar{\eta}, \eta]}\right|_{\substack{\bar{\eta}=0 \\
\eta=0}} \\
& =\frac{\int \mathcal{D} U \operatorname{wick}(\mathcal{O}) \operatorname{det}\left(\mathrm{Q}^{-1}(\mathcal{U})\right) e^{-S_{g}}}{\int \mathcal{D} U \operatorname{det}\left(\mathrm{Q}^{-1}(\mathcal{U})\right) e^{-S_{g}}}
\end{aligned}
$$


where the variational derivative has been suitably defined over Grassmann variables. Note the analogy of the quantum expectation value above to a weighted statistical average.

The non-local nature of the fermion determinant $\operatorname{det}\left(\mathrm{Q}^{-1}(\mathcal{U})\right)$ significantly increases the numerical effort in any numerical simulation. In the quenched approximation this determinant is neglected by setting it identically equal to one. This has the effect of neglecting fermion loops.

In many areas of hadronic physics neglecting fermion loops appears to have a minimal impact on the physics described and to first approximation it can be compensated for by a finite renormalization of the effective coupling constant. The quenched approximation was used in all numerical simulations reported in this dissertation. 


\section{Chapter 3. \\ Weak Interaction Fundamentals}

\section{s.1. Motivation}

Our goal is to compute $S$ matrix elements of the form $\langle f|\mathcal{S}-1| i\rangle$ where the initial state $|i\rangle$ and the final state $\langle f|$ consist of hadrons and the interaction term involves the interplay of the weak and strong interactions as described by the Standard Model of particle physics. A first principles calculation of such a matrix element is extremely difficult since the experimentally accessible initial and final states are QCD bound states involving QCD effects on energy scales of the order of $\Lambda_{Q C D}$ and these effects are by nature non-perturbative.

While low energy QCD effects demand a non-perturbative calculation, effects at and above the energy scale of the $\mathrm{W}$ boson can be treated perturbatively due to asymptotic freedom in QCD. The Operator Product Expansion is used to unravel effects on the two very different energy scales $\mu \approx \Lambda_{Q C D}$ and $\mu \approx m_{w}$ and write weak matrix elements in terms of an effective weak Hamiltonian with the $\mathrm{W}$ boson eliminated as a dynamical field. The effective Hamiltonian is formulated in terms of composite operators built from quark field operators. This effective weak Hamiltonian takes the form

$$
\mathcal{H}_{w}^{e f f}=\sum_{i} c_{i}(\mu) \mathcal{O}_{i}(\mu)
$$

The $c_{\boldsymbol{i}}(\mu)$ 's, contain the details of the short distance physics, calculated perturbatively in QCD and then "improved" using the Renormalization Group Equation. 
Matrix elements of the effective operators carry information about the long distance physics. This separation of short distance and long distance effects, while of great utility, is not the whole story. One still needs a method to calculate matrix elements of the effective operators. It is in these matrix elements where nonperturbative effects are most strongly felt. In both continuum theories and lattice theory the task is then to evaluate matrix elements of these effective operators. Lattice QCD is the ideal framework with which to calculate these matrix elements since it manifestly includes effects to all orders in the QCD coupling constant.

The following sections outline the general formalism of effective weak operators in both the continuum and on the lattice. This overview follows the development given in previous works $24-27,23$.

\subsection{The Continuum Effective weak Hamiltonian}

In the Standard Model the W boson couples to the three families of quarks via a charged current of the $V-A$ form given by

$$
J^{\mu \dagger}=\frac{g_{w}}{2 \sqrt{2}}\left(\begin{array}{lll}
\overline{\mathrm{u}} & \overline{\mathrm{c}} & \overline{\mathrm{t}}
\end{array}\right) \gamma^{\mu}\left(1-\gamma^{5}\right) V_{K M}\left(\begin{array}{l}
\mathrm{d} \\
\mathrm{s} \\
\mathrm{b}
\end{array}\right)
$$

where $g_{w}$ is the weak coupling constant and where $V_{K M}$ is the Kobayashi-Maskawa (KM) matrix. The elements of the unitary matrix $V_{K M}$ are denoted by

$$
V_{K M}=\left(\begin{array}{lll}
V_{u d} & V_{u s} & V_{u b} \\
V_{c d} & V_{c s} & V_{c b} \\
V_{t d} & V_{t s} & V_{t b}
\end{array}\right)
$$

These elements are not determined from within the Standard Model. They can be parameterized by three real angles and one complex phase which are presumably determined by currently unknown physics external to the Standard Model. 
Coupling the $\mathrm{W}$ boson to the current in Eq. (3.2.1) leads to the interaction Lagrangian term

$$
L_{w e a k}^{\text {int }}=\int d^{4} x J^{\mu \dagger}(x) W_{\mu}^{+}(x)+\text { h.c. }
$$

This is then the starting point for a perturbative expansion in powers of $g_{w}$ since for an energy scale characteristic of the momentum transfer in a hadronic matrix element, the $\mathrm{W}$ boson is highly virtual and $g_{w}^{2} / M_{w}^{2}$ forms a small expansion parameter.

To lowest order, a hadronic matrix element involves the exchange of one virtual $\mathrm{W}$ boson. The $\mathrm{S}$ Matrix element for such a process is

$$
\mathcal{A}_{f_{i}}(2 \pi)^{4} \delta^{4}\left(p_{f}-p_{i}\right)=\int d^{4} x d^{4} y D\left(x ; m_{w}\right)\left\langle f\left|T J^{\mu^{\dagger}}(y+\mathrm{x} / 2) J_{\mu}(y-\mathrm{x} / 2)\right| i\right\rangle
$$

where the $\mathrm{W}$ propagator in position space

$$
i g^{\mu \nu} D\left(x_{2}-x_{1} ; m_{w}\right)=\int \frac{d^{4} p}{(2 \pi)^{4}} \frac{-i g^{\mu \nu}}{p^{2}-m_{w}^{2}+i \epsilon} e^{i p\left(x_{2}-x_{1}\right)}
$$

is written in Feynman-'t Hooft gauge.

Since the $\mathrm{W}$ boson mass is so much greater than the momentum transfer typical of the arnplitude, the distance $\mathrm{x}$ in Eq. (3.2.4) over which the $\mathrm{W}$ boson propagates is small relative to a length scale typical of the hadrons. The point to point interaction mediated by the $\mathrm{W}$ boson will appear, to a first approximation, to be a local interaction.

The Operator Product Expansion

$$
T\left(J^{\mu^{\dagger}}(y+\mathrm{x} / 2) J_{\mu}(y-\mathrm{x} / 2)\right) \underset{x \rightarrow 0}{\longrightarrow} \sum_{i} f_{i}(x, \mu, g(\mu)) \mathcal{O}_{i}(y ; \mu)
$$

can then be used to remove the dynamics of the $\mathrm{W}$ boson. 
The Operator Product Expansion replaces time-ordered product of the two charged currents by a sum over a series of local operators. The singular nature of the time-ordered product as $x \rightarrow 0$ is now contained in the c-number coefficients $f_{i}$. The operators $\mathcal{O}_{i}$ are composite local operators renormalized to scale $\mu$.

Substituting Eq. (3.2.6) into Eq. (3.2.4) and doing the integrals yields

$$
\mathcal{A}_{f i}=\sum_{i} \frac{c_{i}\left(m_{w} / \mu, g(\mu)\right)}{m_{w}^{\left(d_{i}-4\right)}}\left\langle f\left|\mathcal{O}_{i}(0 ; \mu)\right| i\right\rangle
$$

The constants $c_{i}$ represent the result of doing the integral over $x$ in Eq. (3.2.4). Dimensional analysis has been used to make the coefficients $c_{i}$ dimensionless by factoring out the proper number of powers of $m_{w}$. Here $d_{i}$ represents the engineering dimension of operator $\mathcal{O}_{i}$.

In Eq. (3.2.7) one sees the separation of the short distance plyysics contained in the $c_{i}$ 's from the long range physics contained in the matrix elements of the effective operators $\mathcal{O}_{i}$.

Since Eq. (3.2.6) is an operator relation, the coefficients $c_{i}$ can be evaluated between any convenient initial and final states. The short distance behavior of these coefficients are of concern and it is on short distance scales where perturbation theory can be applied to QCD. One then takes unbound quarks as initial and final states and treats QCD perturbatively. In charm decays one is interested in amplitudes that on the quark level contain the process $c \rightarrow s u \bar{d}$. In figure 3.2.1 is the tree level diagram along with one gluon loop diagrams contributing the coefficients $c_{i}$ entering in equation 3.2.7.

Although only order $g^{2}$ contributions to the Wilson coefficients are included so far, there is no theoretical barrier to extending the calculation to higher orders in $g^{2}$. 


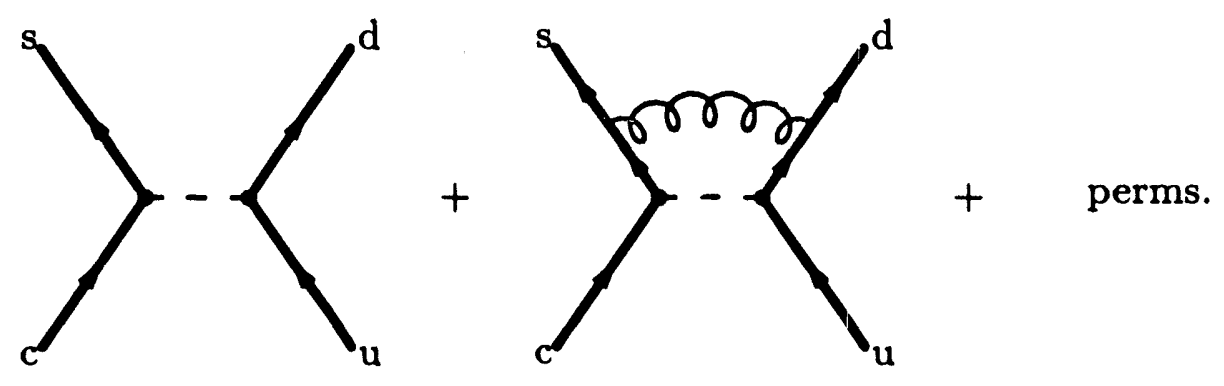

Figure 3.2.1: $1+\operatorname{order}\left(g^{2}\right)$ contributions to $c \rightarrow s u \bar{d}$.

Schematically, contributions of the diagrams in Fig. 3.2.1 yield

$$
\mathcal{A}(\mathrm{c} \rightarrow \mathrm{s} \mathrm{u} \ddot{\mathrm{d}}) \underset{\mu \approx m_{w}}{\longrightarrow} \frac{g_{w}^{2}}{8 m_{w}^{2}} V_{c s}^{*} V_{u d}\left\{\left[1+O\left(g^{2}(\mu)\right)\right] \mathcal{O}_{1}^{\text {bare }}+O\left(g^{2}(\mu)\right) \mathcal{O}_{2}^{\text {bare }}\right\}
$$

where

$$
\begin{aligned}
& \mathcal{O}_{1}^{\text {bare }}=\overline{\mathrm{s}}^{a} \gamma^{\mu}\left(1-\gamma^{5}\right) \mathrm{c}^{a} \overline{\mathrm{u}}^{b} \gamma^{\mu}\left(1-\gamma^{5}\right) \mathrm{d}^{b} \\
& \mathcal{O}_{2}^{\text {bare }}=\overline{\mathrm{s}}^{a} \gamma^{\mu}\left(1-\gamma^{5}\right) \mathrm{d}^{a} \overline{\mathrm{u}}^{b} \gamma^{\mu}\left(1-\gamma^{5}\right) \mathrm{c}^{b} .
\end{aligned}
$$

Operator $\mathcal{O}_{1}$ is the four-fermion (dimension 6) operator of the $\left(V^{\mu}-A^{\mu}\right) \otimes$ $\left(V^{\mu}-A^{\mu}\right)$ form one would expect by naively shrinking the $\mathrm{W}$ boson propagator down to a point. QCD corrections have caused $\mathcal{O}_{1}$ to mix with a new operator $\mathcal{O}$.. Examining the explicit color indices $(a, b)$ in Eq. (3.2.9) one sees that operator $\mathcal{O}_{2}$ is not simply the Fierz rearrangement of $\mathcal{O}_{1}$ since both $\mathcal{O}_{1}$ and $\mathcal{O}_{2}$ are diagonal in color and the Fierz rearrangement would mix color indices. Operators $\mathcal{O}_{1}$ and $\mathcal{O}_{2}$ individually are not multiplicatively renormalizable. However, the linear combinations

$$
\mathcal{O}_{ \pm}^{\text {bare }}=\frac{1}{2}\left[\mathcal{O}_{1}^{\text {bare }} \pm \mathcal{O}_{2}^{\text {bare }}\right]
$$

are multiplicatively renormalizable. Thus,

$$
\mathcal{O}_{ \pm}(0 ; \mu)=Z_{ \pm}\left(\Lambda / \mu, g_{0}\right) \mathcal{O}_{ \pm}^{\text {bare }}(0)
$$


where the renormalization constants $Z_{ \pm}$depend on the cutoff introduced in the calculation of the diagrams of Fig. 3.2.1.

Examining Eq. (3.2.8) it is possible to extract the leading order expressions for $c_{ \pm}$

$$
c_{ \pm}\left(m_{w / \mu} \approx 1, g\left(m_{w}\right)\right)=\frac{g_{w}^{2}}{8} V_{c s}^{*} V_{u d}\left[1+O\left(g^{2}(\mu)\right)\right]
$$

These coefficients are found to obey the Renormalization Group Equation. Note that the invariant amplitude Eq. (3.2.7) must be independent of the scale $\mu$. Thus,

$$
\mu \frac{d}{d \mu} \mathcal{A}_{f i}=0
$$

and then it can be shown that

$$
\left[\mu \frac{\partial}{\partial \mu}+\beta(g) \frac{\partial}{\partial g}+\gamma_{ \pm}(g)\right] c_{ \pm}\left(m_{w / \mu}, g(\mu)\right)=0
$$

where $\beta(g)$ is the QCD beta function and $\gamma_{ \pm}$are the anomalous dimensions of operators $\mathcal{O}_{ \pm}$.

The coefficients in Eq. (3.2.7) have potentially troublesome factors of the form $\log \left(m_{w} / \mu\right)$ arising in order $g^{2}$ and higher terms. As one goes from $\mu \approx m_{w}$ towards smaller $\mu$, the higher order terms can become important. Using the Renormalization Group Equation (RGE) it is possible to evolve the coefficients from an energy scale $\mu \approx m_{w}$ down to a lower energy scale. This is possible because the solution of Eq. (3.2.14) involves a summation of these logarithmic terms to all orders.

The anomalous dimensions of operators $\mathcal{O}_{ \pm}$are defined by

$$
\gamma_{ \pm} \equiv \mu \frac{d}{d \mu} \log Z_{ \pm}
$$


where $Z_{ \pm}$are the operator renormalization coefficients in Eq. (3.2.11).

The function $\beta(g)$ to two loop order is given by

$$
\beta(g) \equiv \mu \frac{\partial}{\partial \mu} g(\mu)=-b_{0} \frac{g^{3}}{16 \pi^{2}}-b_{1} \frac{g^{5}}{\left(16 \pi^{2}\right)^{2}}+\ldots
$$

where the running coupling constant squared $g^{2}$ is given by

$$
g^{2}(\mu)=\frac{16 \pi^{2}}{b_{0} \log \left(\mu^{2} / \Lambda^{2}\right)}\left[1-\frac{b_{1} \log \log \left(\mu^{2} / \Lambda^{2}\right)}{b_{0}^{2} \log \left(\mu^{2} / \Lambda^{2}\right)}+\ldots\right]
$$

The coefficients $b_{0}$ and $b_{1}$ are

$$
\begin{aligned}
& b_{0}=\frac{11}{3} N_{c}-\frac{2}{3} N_{f} \\
& b_{1}=\frac{34}{3} N_{c}^{2}-\frac{10}{3} N_{c} N_{f}-\frac{\left(N_{c}^{2}-1\right)}{N_{c}} N_{f}
\end{aligned}
$$

where $N_{c}$ is the number of colors for a $S U\left(N_{c}\right)$ type gauge symmetry and $N_{f}$ is the number of effective flavors of quarks. For continuum QCD, $N_{c}=3$ and below the b quark mass $N_{f}=4$. Although the beta function is given here to two loop order, since we are working to one loop order in Fig. 3.2.1, only the one loop term of the beta function will be used in Eq. (3.2.14).

The RGE has the solution

$$
c_{ \pm}\left(m_{w / \mu}, g(\mu)\right)=c_{ \pm}\left(1, g\left(m_{w}\right)\right) \exp \int_{g(\mu)}^{g\left(m_{w}\right)} d g^{\prime} \frac{\gamma_{ \pm}\left(g^{\prime}\right)}{\beta\left(g^{\prime}\right)}
$$

which can be verified by inserting this equation into Eq. (3.2.14). The lower limit of the integral is selected to be $\mu \approx m_{c} \approx 1.5 \mathrm{GeV}$ thereby setting the energy scale to be appropriate to charm decays. 


\begin{tabular}{|c|c|c|}
\hline$\mu(\mathrm{GeV})$ & $\overline{c+(\mu)}$ & $\overline{c-(\mu)}$ \\
\hline 80.6 & 1 & 1 \\
\hline 40.0 & $.974\left(\begin{array}{l}-3 \\
+2\end{array}\right)$ & $1.054\left(\begin{array}{ll}+ & 6 \\
-\end{array}\right)$ \\
\hline 10.0 & $.913\left(\begin{array}{l}-10 \\
+8\end{array}\right)$ & $1.199\left(\begin{array}{l}+28 \\
-20\end{array}\right)$ \\
\hline 5.0 & $.877\left(\begin{array}{l}-17 \\
+12\end{array}\right)$ & $1.301\left(\begin{array}{c}51 \\
-34\end{array}\right)$ \\
\hline 1.6 & $.799\left(\begin{array}{c}-38 \\
+24\end{array}\right)$ & $1.565\left(\begin{array}{l}+161 \\
-89\end{array}\right)$ \\
\hline 1.5 & $.794\left(\begin{array}{l}-40 \\
+25\end{array}\right)$ & $1.587\left(\begin{array}{c}+174 \\
-95\end{array}\right)$ \\
\hline 1.4 & $.788\left(\begin{array}{l}-43 \\
+26\end{array}\right)$ & $1.611\left(\left(_{-101}^{+198}\right)\right.$ \\
\hline
\end{tabular}

Table 3.2.1: Values of the Wilson Coefficients as a function of the energy scale $\mu$. These values are for $N_{c}=3$ and $N_{f}=4$. The range of each value reflects the pessible range of $\Lambda \frac{(4)}{M S}=.200_{-80}^{+150} \mathrm{GeV}$.

The anomalous dimensions to one loop order of operators $\mathcal{O}_{ \pm}$are found to be

$$
\begin{aligned}
& \gamma_{+}=-4 \frac{g^{2}}{16 \pi^{2}} \\
& \gamma_{-}=8 \frac{g^{2}}{16 \pi^{2}}
\end{aligned}
$$

Redefining $c_{ \pm}$so that $c_{ \pm}\left(\mu \approx m_{w}\right)=1$ by removing an overall common factor of $g_{w}^{2} V_{c s}^{*} V_{u d} / 8$ and using Eq. (3.2.19),

$$
\begin{aligned}
& c_{+}\left(m_{w / \mu}, g(\mu)\right)=\left(\frac{g(\mu)}{g\left(m_{w}\right)}\right)^{-4 / b_{0}} \\
& c_{-}\left(m_{w / \mu}, g(\mu)\right)=\left(\frac{g(\mu)}{g\left(m_{w}\right)}\right)^{8 / b_{0}}
\end{aligned}
$$

Table 3.2.1 shows numerical values for $c_{ \pm}$as they are evolved from $\mu=m_{w}$ down to $\mu \approx m_{c}$. The values shown correspond to $N_{c}=3$ and an effective number of flavors of four quarks $\left(N_{f}=4\right)$. The best value and range given for the coefficients are calculated based on the best value and range $\Lambda \frac{(4)}{M S}=.200_{-80}^{+150} \mathrm{GeV}$ for $\Lambda_{Q C D}$ as given in Ref. 3 . 
Combining the common factor removed in the redefinition of $c_{ \pm}$with the factor of $m_{w}^{-2}$ from Eq. (3.2.7) and defining the Fermi Constant

$$
G_{f} \equiv \frac{g_{w}^{2}}{2 \sqrt{2} m_{w}^{2}}
$$

the effective weak interaction Hamiltonian becomes

$$
\mathcal{H}_{\text {weak }}^{e f f}=\frac{G_{f} V_{c s}^{*} V_{u d}}{2 \sqrt{2}}\left\{c_{+}(\mu) \mathcal{O}_{+}(\mu)+c_{-}(\mu) \mathcal{O}_{-}(\mu)\right\}
$$

This effective Hamiltonian is the starting point for the continuum weak matrix element calculations such as the vacuum saturation approximation, large $N_{c}$ methods and QCD sum-rule methods.

\subsection{Lattice Effective Weak Hamiltonian}

Field theory defined on the lattice has an ultraviolet cutoff due to the discreteness of space-time points. For current lattices used in weak matrix element calculations, this cutoff lies well below the $\mathrm{W}$ boson mass. It is then natural to use a theory with effective weak interactions in which the $\mathrm{W}$ boson is no longer a dynamical field just as in the previous section. Using the continuum results of the previous section, define an effective weak Hamiltonian at the scale of the lattice cutoff of around $\mu \approx \pi / a$. The effective operators of the continuum theory are the starting point for lattice calculations.

The continuum calculation of the previous section used dimensional regularization and the $\overline{M S}$ renormalization scheme (i.e. absorb the divergent pole piece and a factor of $-\gamma_{E}+\log \sqrt{4 \pi}$ in the counter-terms). On the lattice the regulator is the lattice spacing $a$ and the renormalization scheme is not the same as the 
continuum scheme. The lattice regularized operators must then be matched to their continuum counterparts. This matching is done by comparing a continuum perturbative expansion for the effective operators with a perturbative expansion for the operators done using lattice perturbation theory.

The relation of continuum operators to their lattice counterparts is

$$
\mathcal{O}_{i}^{\text {cont }}(\mu)=\mathcal{Z}_{i j}^{\text {match }}(a \mu, g(\mu)) \mathcal{O}_{j}^{\text {lat }}(a, r)
$$

where $a$ is the lattice spacing and $r$ is the Wilson parameter appearing in the fermion lattice action Eq. (2.5.1).

Working to order $g^{2}$ Eq. (3.3.1) becomes

$$
\mathcal{O}_{i}^{\text {cont }}(\mu)=\left(\delta_{i j}+\frac{g^{2}}{16 \pi^{2}} Z_{i j}(a \mu, r)\right) \mathcal{O}_{j}^{l a t}(a, r)
$$

The relation between matrix elernents of renormalized and bare quantities in either continuum or lattice regularized schemes to order $g^{2}$ is

$$
\left\langle f\left|\mathcal{O}_{i}\right| i\right\rangle^{r e g}=\left(\delta_{i j}+\frac{g^{2}}{16 \pi^{2}} \rho_{i j}^{r e g}(p)\right)\left\langle i\left|\mathcal{O}_{j}\right| f\right\rangle^{0} \quad \text { reg = cont., lat. }
$$

where the external states carry momentum $p$. Substituting these relations into Eq. (3.3.2),

$$
Z_{i j}(a \mu, r)=\rho_{i j}^{\text {cont }}(p)-\rho_{i j}^{l a t}(p, a \mu, r)
$$

The matching condition is found by comparing function $\rho_{i j}^{\text {cont }}$ computed in continuum perturbation theory with the same quantity computed in lattice perturbation theory.

The Feynman rules of lattice perturbation theory differ from the usual Feynman rules of continuum QCD because on the lattice the discrete nature of space-time 
introduces an explicit regulator and thereby a new parameter the finite lattice spacing $a$. These lattice Feynman rules lead to the same four-fermion $(V-A)^{\mu} \otimes$ $(V-A)^{\mu}$ operators of the form

$$
\mathcal{O}_{ \pm}=\frac{1}{2}\left[\bar{\psi}_{1} \gamma^{\mu}\left(1-\gamma^{5}\right) \psi_{2} \bar{\psi}_{3} \gamma^{\mu}\left(1-\gamma^{5}\right) \psi_{4} \pm(2 \leftrightarrow 4)\right]
$$

as is the case in the continuum. However, these four-fermion operators can now mix with additional four-fermion operators not of the $(V-A)^{\mu} \otimes(V-A)^{\mu}$ form and, where possible, with lower dimensional operators. These lower dimensional operators naively must depend on inverse powers of the lattice spacing by dimensional analysis.

Equation 3.3.2 becomes

$$
\begin{aligned}
\mathcal{O}_{ \pm}^{\text {cont }}(\mu)=[1+ & \left.\frac{g^{2}}{16 \pi^{2}} Z_{ \pm}(r, a \mu)\right] \mathcal{O}_{ \pm}^{\text {lat }} \\
& +\frac{g^{2}}{16 \pi^{2}}\left[r^{2} Z^{*}(r) \mathcal{O}_{ \pm}^{\text {lat }}+Z_{i}^{(d<6)}(r, a, \mu) \mathcal{O}_{i}^{(d<6)}\right]
\end{aligned}
$$

where $\mathcal{O}_{ \pm}^{\text {cont }, \text { lat }}$ have the form shown in Eq. (3.3.5).

The coefficients $Z_{ \pm}$have the form

$$
Z_{ \pm}(r, a \mu)=\left(\gamma_{ \pm}^{(1)} \log (a \mu)+Z_{ \pm}(r, 1)\right)
$$

where $\gamma_{ \pm}=\frac{g^{2}}{16 \pi^{2}} \gamma_{ \pm}^{(1)}$. Values for $Z_{ \pm}(r, 1)$ and $r^{2} Z^{*}(r)$ are tabulated in Reference 27. For $r=1$, the values in the so called $\overline{E Z}$ dimensional reduction renormalization scheme are

$$
\begin{aligned}
& Z_{+}(1,1)=-50.174 \\
& Z_{-}(1,1)=-4.5 .308 \\
& r^{2} Z^{*}(1)=9.6431
\end{aligned}
$$


The symbols $\mathcal{O}_{ \pm}^{\prime}$ represents a linear combination of so called "off-diagonal" four-fermion operators

$$
\mathcal{O}_{ \pm}^{\prime}=\sum_{i} a_{i}\left(\bar{\psi}_{1} \Gamma^{i} \psi_{2} \bar{\psi}_{3} \Gamma^{i} \psi_{4} \pm(2 \leftrightarrow 4)\right)
$$

where $\Gamma^{i}$ is an element of the Dirac algebra

$$
\Gamma^{i} \in\left\{1, \gamma^{\mu}, \sigma^{\mu \nu}, \gamma^{\mu} \gamma^{5}, \gamma^{5}\right\}
$$

Note that the $\mathcal{O}_{ \pm}^{\prime}$ terms are all even under parity even though the naive fourfermion operator contains both even and odd parity parts. These off-diagonal parity even terms can then be safely neglected when calculating amplitudes such as $D \rightarrow K \pi$ where a pseudoscalar $\left(J^{P}=0^{-}\right)$decays into two pseudoscalars since only parity changing operators will contribute.

The lower dimensional operators $\mathcal{O}_{i}^{(d<6)}$ occur when quark fields in an operator can contract with one another. For example, suppose

$$
\bar{\psi}_{1}=\overline{\mathrm{d}} \quad \psi_{2}=\mathrm{c} \quad \bar{\psi}_{3}=\overline{\mathrm{u}} \quad \psi_{4}=\mathrm{d}
$$

then the lower-dimensional operators are

$$
\sum_{i} Z_{i}^{(d<6)}(r, a, \mu) \mathcal{O}_{i}^{(d<6)}=z_{3} \overline{\mathrm{u}} \mathrm{c}+z_{3}^{\prime} \overline{\mathrm{u}} \gamma^{5} \mathrm{c}+z_{5} \overline{\mathrm{u}} \sigma^{\mu \nu} G^{\mu \nu} \mathrm{c}
$$

These operators arise from considering diagrams like those shown in figure 3.3.1. On dimensional grounds the coefficients of these operators are proportional to powers of $a^{-1}$. As $a \rightarrow 0$ the individual coefficients of these operators grow yet the sum Eq. (3.3.12) must approach zero. In addition, non-perturbative effects may 

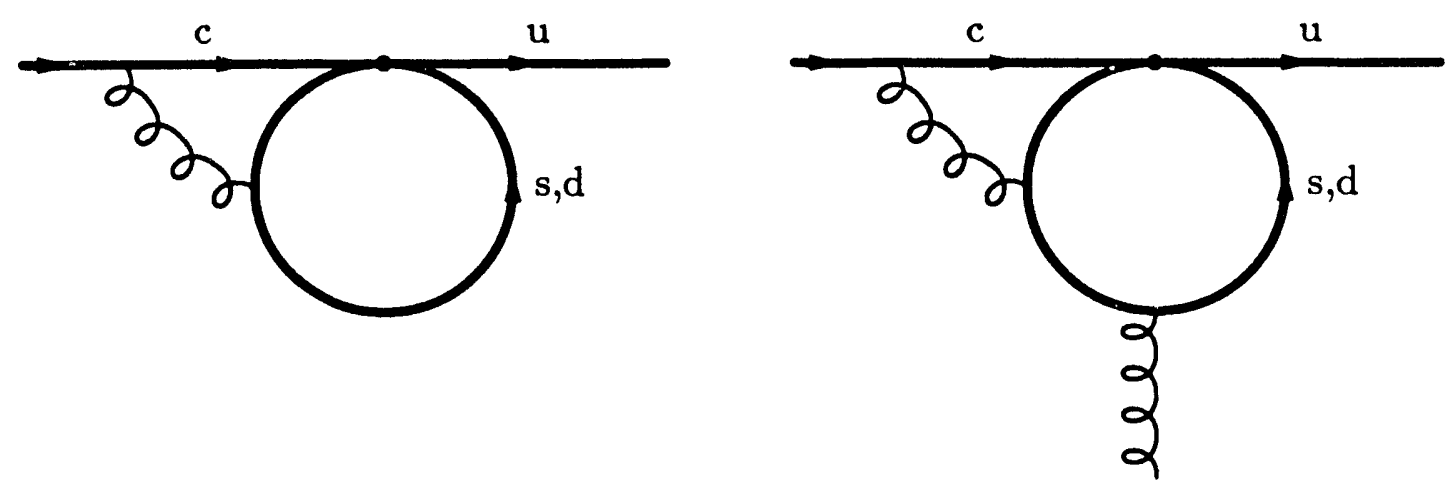

Figure 3.3.1: Diagrams that lead to mixing of dim. 6 operators with lower dimension operators

make a perturbative evaluation of the coefficients $z_{3}, z_{3}^{\prime}$, and $z_{5}$ totally unreliable. A non-perturbative treatment of these operators is then required.

In the $D \rightarrow K \pi$ decays that are of main interest in this work the mixing from lower dimensional operators does not occur in either Cabbibo allowed or doubly Cabbibo suppressed decay modes since for in these cases none of the quark operators within the effective weak operator can contract with another.

The final form of the lattice effective weak Hamiltonian is

$$
\mathcal{H}_{\text {weak }}^{e f f}=\frac{G_{f} V_{21}^{*} V_{34}}{2 \sqrt{2}}\left\{c_{+}(\mu) \mathcal{O}_{+}(\mu)+c_{-}(\mu) \mathcal{O}_{-}(\mu)\right\}
$$

where $\mathcal{O}_{ \pm}$are the continuum operators ofEq. (3.3.5) and $V_{21}$ and $V_{34}$ are the elements of the KM matrix connecting quarks of flavor 1 to 2 and of flavor 3 to 4 respectively.

The continuum coefficients $c_{ \pm}(\mu)$ are computed as in Eq. (3.2.21) except that now $N_{f}=0$ since the lattice calculation is done in the quencled approximation and there are no fermion loop contributions to $g(\mu)$.

The coupling constant is computed using $\Lambda \frac{(0)}{M S}$ determined from the relation

$$
\Lambda_{\overline{M S}}=28.8 \Lambda^{\text {lat }}
$$




\begin{tabular}{|c|c|c|c|c|}
\hline$a^{-1}(\mathrm{GeV})$ & $\mu(\mathrm{GeV})$ & $\Lambda_{\frac{(0)}{M S}}^{(\mathrm{GeV})}$ & $c_{+}^{\text {lat }}(\mu, \Lambda)$ & $c_{-}^{\text {lat }}(\mu, \Lambda)$ \\
\hline 2.0 & $\frac{1.7 \pi}{a}$ & .135 & .4962 & .7174 \\
\hline 2.0 & $\frac{\pi}{a}$ & .135 & .4648 & .6466 \\
\hline 2.0 & $\frac{2 \pi}{a}$ & .135 & .5048 & .7364 \\
\hline
\end{tabular}

Table 3.3.1: Variation of the Lattice Wilson Coefficients with the inverse lattice spacing and the energy scale $\mu$ for $\beta=6.0$. The first row shows the nominal values.

for $S U(3)$.

Using the beta value for the lattice, the lattice spacing $a$ and

$$
a=\frac{1}{\Lambda^{l a t}}\left(\frac{N_{c} b_{0}}{8 \pi^{2} \beta}\right)^{-\frac{b_{1}}{2 b_{0}^{2}}} \exp \left(-\frac{4 \pi^{2}}{N_{c} b_{0}^{2}} \beta\right)\left[1+o\left(\frac{1}{\beta}\right)\right]
$$

$\Lambda^{\text {lat }}$ is determined.

Based on a suggestion by Mackenzie ${ }^{28}$ the value selected as the best estimate for the energy scale where the perturbative matching to the continuum has taken place is ${ }^{29}$,

$$
\mu=\frac{1.7 \pi}{a} .
$$

Table 3.3.1 shows the variation of the quantities

$$
c_{ \pm}^{\text {lat }}(a \mu, \Lambda \overline{M S})=c_{ \pm}^{\text {cont }}(\mu, \Lambda)\left[1+\frac{g^{2}}{16 \pi^{2}} Z_{ \pm}(1, a \mu)\right]
$$

with choice of $\mu$ at $\beta=6.0$. To order $g^{2}$, quantities $c_{ \pm}^{\text {lat }}$ should be independent of the exact choice of $\mu$. The variation is about $13 \%$ for the range of $\mu$ shown. 


\section{Chapter 4.}

\section{Lattice Correlation Functions}

\subsection{Interpolating Fields}

There is no unique correspondence between particles and fields. Physical quantities such as decay amplitudes can be extracted from Green functions expressed in terms of field operators other than the operators that obey the normal canonical (anti)commutation relations. Given some particle we construct correlation functions involving this particle from time-ordered products of field operators $\chi$ selected to represent the particle. A necessary requirement for such an interpolating field is that it have a finite overlap with the single particle state of the particle in question. That is if

$$
\left\langle 0\left|\chi_{\pi}(0)\right| \pi(k)\right\rangle \neq 0
$$

then $\chi_{\pi}$ is a perfectly good interpolating field for particle $\pi$ where $|\pi(k)\rangle$ is a single particle state for particle $\pi$. Thus decay amplitudes involving composite particles can be extracted from correlation functions built from interpolating fields even though these composite particles are QCD bound states of quarks for which the canonical field operators are not known.

All interpolating fields are not created equally in that some choices for $\chi(x)$ are better than others. A practical criterion for picking these interpolating fields is that the overlap as in Eq. (4.1.1) with the physical particle state of interest be maximized while the coupling to radial excitations of the particle be small. For a meson this means that among the properties an interpolating field should have 
are that it be dominantly a color singlet with the same spin and parity behavior as the meson and that it have the same valence quark content as the meson.

For mesons such interpolating fields can be written with the general form

$$
\chi(x)=\frac{1}{M^{2}} \int d^{4} x_{1} d^{4} x_{2} \overline{\mathrm{q}}_{1}^{a}\left(x_{1}\right) \phi^{a b}\left(x ; x_{1}, x_{2}\right) \Gamma \mathrm{q}_{2}^{b}\left(x_{2}\right)
$$

where the particle consists of valence quarks flavors $\bar{q}_{1}$ and $q_{2}$ with color indices $a$ and $b$. The spin and parity properties are specified by the Dirac matrix $\Gamma$ and the explicit factor of $1 / M^{2}$ makes function $\phi$ dimensionless.

As an example, the pseudoscalar pion $\pi^{+}\left(J^{P}=0^{-}\right)$has $\Gamma=-i \gamma^{5}$ with $\bar{q}_{1}=\overline{\mathrm{d}}$ and $\mathrm{q}_{2}=\mathrm{u}$. The simplest choice for the function $\phi^{a b}\left(x ; x_{1}, x_{2}\right)$ is

$$
\phi^{a b}\left(x ; x_{1}, x_{2}\right)=\delta^{a b} \delta^{4}\left(x-x_{1}\right) \delta^{4}\left(x-x_{2}\right) .
$$

for which, the $\pi^{+}$interpolating field becomes

$$
\chi_{\pi^{+}}(x)=-i \overline{\mathrm{d}}(x) \gamma^{5} \mathrm{u}(x)
$$

where we have dropped the $1 / M^{2}$ factor.

Since the two quark field operators are at the same space-time point there is no need to connect the two operators by a "gluon string" consisting of some path-ordered product of $U$ matrices in order to maintain gauge invariance. This is the form of the interpolating fields used in this work. For pseudoscalar particles such as the pion, kaon, and the $\mathrm{D}$, or for vector particles such as $K^{*}$, this is a reasonable choice since for such states the quark and anti-quark have relative orbital angular momentum $L=0$ and there should be significant overlap of the two quarks. For scalar $\left(0^{+}\right)$particles such as $K_{0}^{*}$ the point-like interpolating operator 
$\chi_{K_{0}^{*}}(x)=\overline{\mathrm{s}}(x) \mathrm{d}(x)$ does not couple as well to the physical state since in such a state the two quarks have relative angular momentum $L=1$.

Intuitively, coupling to the lowest particle state can be enhanced and coupling to higher energy radial excitations can be diminished by choosing the function $\phi^{a b}\left(x ; x_{1}, x_{2}\right)$ so that the interpolating fields have a spread in $x$ that is of the same order of magnitude as the size of the particle of interest.

Perhaps a better choice is $\phi^{a b}\left(x ; x_{1}, x_{2}\right)=\delta^{a b} \delta\left(t-t_{1}\right) \delta\left(t-t_{2}\right)$ where for simplicity the operator is smeared over the whole volume at a given time. The increase in number of source points beyond a single delta function source should then lead to an increase in measured signal. However, since the two quarks are not connected by a gluon string, the gauge fields should be put in Coulomb gauge to reduce noise due the gauge variant part of this operator. Using "Wall Operators" of this type would be especially useful for scalar particles.

\subsection{Pseudoscalar Two-Point Functions}

An example of a two-point correlation function is the $\pi^{+}$propagator. The propagator with lattice three-momentum $\vec{p}$ from the origin to the space-time point $x=(\vec{x}, t)$ is

$$
G_{\pi^{+}}^{(2)}(t, 0 ; \vec{p})=\sum_{\vec{x}} e^{\frac{2 \pi i}{L} \vec{p} \cdot \vec{x}}\left\langle 0\left|\mathrm{~T}\left(\chi_{\pi^{+}}(x) \chi_{\pi^{+}}^{\dagger}(0)\right)\right| 0\right\rangle
$$

Using the interpolating field of Eq. (4.1.4) and taking $t>0$, this expression becomes

$$
G_{\pi^{+}}^{(2)}(t, 0 ; \vec{p})=-\sum_{\vec{x}} e^{\frac{2 \pi i}{L} \vec{p} \cdot \vec{x}}\left\langle 0\left|\overline{\mathrm{d}} \gamma^{5} \mathrm{u}(x) \overline{\mathrm{u}} \gamma^{5} \mathrm{~d}(0)\right| 0\right\rangle
$$


The quark fields are then Wick contracted and the Hermiticity property of quark propagators Eq. (2.6.4) is used to get

$$
G_{\pi^{+}}^{(2)}(t, 0 ; \vec{p})=\sum_{\vec{x}} e^{\frac{2 \pi i}{L} \vec{p} \cdot \vec{x}}\left\langle\operatorname{Tr}_{s c}\left(\mathrm{D}^{\dagger}(x, 0 ; \mathcal{U}) \mathrm{U}(x, 0 ; \mathcal{U})\right)\right\rangle
$$

where $\mathrm{U}(x, 0 ; \mathcal{U})$ and $\mathrm{D}(x, 0 ; \mathcal{U})$ are respectively the $\mathrm{u}$ and $\mathrm{d}$ quark propagators from the origin to space-time point $x$ calculated in a fixed background gauge configuration $\mathcal{U}$ and the trace is over both spin and color indices. In a Monte Carlo simulation the brackets $\langle\ldots\rangle$ signify an average over a representative collection of gauge field configurations $\mathcal{U}$.

It is important to note that it is only necessary to compute the quark propagators via Eq. (4.2.3) starting from the origin to all space-time points rather than from all points to all points. This is fortunate since the latter calculation requires a factor that scales with the volume of the lattice more computer time to calculate numerically.

Products of quark propagators summed over spatial degrees of freedom such as

$$
\sum_{\vec{x}} e^{\frac{2 \pi i}{L} \vec{p} \cdot \vec{x}} D^{\dagger}(x, 0 ; \mathcal{U}) \mathrm{U}(x, 0 ; \mathcal{U})
$$

occur repeatedly in the calculation of lattice correlation functions. Since such quantities are more compact than the quark propagators themselves, and the same ones are frequently needed, considerable numerical effort is saved by creating libraries of frequently used combinations. In the process called "condensing" quantities of the form

$$
\sum_{\vec{x}} e^{\frac{2 \pi i}{L} \vec{p} \cdot \vec{x}} \mathrm{Q}_{2}^{\dagger}(x, 0 ; \mathcal{U}) \Gamma Q_{1}(x, 0 ; \mathcal{U})
$$


are computed and stored in "condensed files" as a function of quark flavors, the Dirac matrix $\Gamma$, the lattice three-momentum $\vec{p}$, the crinfiguration $\mathcal{U}$, and the time $t$. Numerically, lattice correlation functions are then constructed in tinker-toy like fashion from pieces read into the computer from the condensed files.

Returning to Eq. (4.2.1) we examine the behavior of $G_{\pi^{+}}^{(2)}(t, 0)$ at large times. The continuum completeness relationship projecting onio single particle states

$$
1=\sum_{A} \int \frac{d^{3} \vec{k}}{(2 \pi)^{3} 2 E_{A}(\vec{k})}|A(\vec{k})\rangle\langle A(\vec{k})|
$$

becomes on the lattice

$$
1=\frac{1}{L^{3}} \sum_{A \vec{k}} \frac{1}{2 a E_{A}(\vec{k})}|A(\vec{k})\rangle\langle A(\vec{k})|
$$

where the lattice quantities are rescaled to make them dimensionless. Taking $t>0$ and using the completeness relation to insert a complete set of states Eq. (4.2.1) becomes

$$
\begin{aligned}
G_{\pi^{+}}^{(2)}(t, 0 ; \vec{p})= & \frac{1}{L^{3}} \sum_{\vec{x}} \sum_{A \vec{k}} \frac{1}{2 a E_{A}(\vec{k})} e^{\frac{2 \pi i}{L} \vec{p} \cdot \vec{x}} \\
& \left\langle 0\left|\chi_{\pi^{+}}(x)\right| A(\vec{k})\right\rangle\left\langle A(\vec{k})\left|\chi_{\pi^{+}}^{\dagger}(0)\right| 0\right\rangle
\end{aligned}
$$

Translational invariance applied to operator $\chi_{\pi^{+}}(x)$ yields

$$
\chi_{\pi^{+}}(x)=e^{\left(a H t+\frac{2 \pi i}{L} \hat{p} \cdot \vec{x}\right)} \chi_{\pi^{+}}(0) e^{-\left(a H t+\frac{2 \pi i}{L} \hat{p} \cdot \vec{x}\right)} .
$$

Making this substitution and acting on the intermediate and final states with the Hamiltonian $H$ and momentum operator $\hat{p}$ and then using

$$
\sum_{\vec{x}} e^{\frac{2 \pi i}{L} \vec{x} \cdot(\vec{p}-\vec{k})}=L^{3} \delta_{\vec{p}, \vec{k}}
$$


to do the sums over $\vec{x}$ and $\vec{k}_{A}$ yields

$$
G_{x^{+}}^{(2)}(t, 0 ; \vec{p})=\sum_{A} \frac{\left|\left\langle A(\vec{p})\left|\chi_{x^{+}}^{\dagger}(0)\right| 0\right\rangle\right|^{2}}{2 a E_{A}(\vec{p})} e^{-a E_{A}(\vec{p}) t} .
$$

The states that have a non-zero contribution to this sum are the $\pi^{+}$and radial excitations of the pion. The higher energy radial excitations fall off exponentially compared to the pion which will then give the only contribution as $t \rightarrow+\infty$. Thus,

$$
G_{\pi^{+}}^{(2)}\left(t, 0 ; \vec{p}_{\pi}\right) \underset{t \rightarrow \infty}{\longrightarrow} \frac{\left|Z_{\pi}\left(\vec{p}_{\pi}\right)\right|^{2}}{2 a E_{\pi}\left(\vec{p}_{\pi}\right)} e^{-a E_{\pi}\left(\vec{p}_{\pi}\right) t} .
$$

where $E_{\pi}\left(\vec{p}_{\pi}\right)$ is the energy of a pion of three-momentum $\vec{p}_{\pi}$ and $\left|Z_{\pi}\left(\vec{p}_{\pi}\right)\right|^{2}=$ $\left|\left\langle\pi^{+}\left(\vec{p}_{\pi}\right)\left|\chi_{\pi^{+}}^{\dagger}(0)\right| 0\right\rangle\right|^{2}$ measures the overlap of a single particle state created by $\chi^{\dagger}$ with the single particle state normalized according to

$$
\left\langle A(\vec{p}) \mid A\left(\vec{p}^{\prime}\right)\right\rangle=L^{3} 2 a E_{A}(\vec{p}) \delta_{\vec{p}, \vec{p}}
$$

Numerical simulations of pseudoscalar propagators such as for particles pion, kaon and $D$ employ expressions of the type Eq. (4.2.3) to compute the value of $G_{P}^{(2)}\left(t, 0 ; \vec{p}_{\pi}\right)$ as a function of time $t$. The resulting points can then be fitted to Eq. (4.2.12) to extract values for $Z_{\pi}\left(\vec{p}_{\pi}\right)$ and $a E_{\pi}\left(\vec{p}_{\pi}\right)$.

On the lattice operators and states have been rescaled to make them dimensionless and to remove factors of the quark hopping constants. These factors must be put back in before the corresponding continuum quantities can be computed. For fermion fields,

$$
\psi \rightarrow \sqrt{\frac{2 \kappa}{a^{3}}} \psi
$$

which is the rescaling that was done for the lattice fermion action. For interpolating fields of the form $\bar{\psi}_{1} \Gamma \psi_{2}$

$$
\chi \rightarrow \sqrt{\frac{4 \kappa_{1} \kappa_{2}}{a^{2}}} \chi
$$


where Eq. (4.2.14) has been used for the two fermionic fields and there is an extra factor of $a^{2}$ in order that $\chi$ have the correct engineering dimension. Then

$$
G^{(2)}(t, 0) \rightarrow 4 a \kappa_{1} \kappa_{2} G^{(2)}(t, 0)
$$

where one must remember the factor of $a^{3}$ arising from the sum over $\vec{x}$ as in Eq. (4.2.1). Then from the asymptotic form of the propagator Eq. (4.2.12),

$$
|Z|^{2} \rightarrow 4 \kappa_{1} \kappa_{2}|Z|^{2}
$$

\subsection{Scalar Two-Point Functions}

Consider the propagator for a scalar particle with valence quarks $\bar{d} s$. Using the local operator $\chi(x)=\overline{\mathrm{d}} \mathrm{s}(x)$ as an interpolating field and taking $(t>0)$ the scalar propagator becomes

$$
G_{s c l r}^{(2)}(t, 0 ; \vec{p})=\sum_{\vec{x}} e^{\frac{2 \pi i}{L} \vec{p} \cdot \vec{x}}\langle 0|\bar{d} s(x) \bar{s} d(0)| 0\rangle
$$

Doing the Wick contractions and using the Hermiticity property of quark propagators as in the pseudoscalar case yields

$$
\begin{aligned}
& G_{s c l r}^{(2)}(t, 0 ; \vec{p})= \\
& \quad-\sum_{\vec{x}} e^{\frac{2 \pi i}{L} \vec{p} \cdot \vec{x}}\left\langle 0\left|\operatorname{Tr}_{s c}\left(\gamma^{5} \mathrm{D}^{\dagger}(x, 0 ; \mathcal{U}) \gamma^{5} \mathrm{~S}(x, 0 ; \mathcal{U})\right)\right| 0\right\rangle
\end{aligned}
$$

As in the pseudoscalar case, the asymptotic form of the propagator is

$$
G_{s c l r}^{(2)}\left(t, 0 ; \vec{p}_{s c l r}\right) \underset{t \rightarrow \infty}{\longrightarrow} \frac{\left|Z_{s c l r}\left(\vec{p}_{s c l r}\right)\right|^{2}}{2 a E_{s c l r}\left(\vec{p}_{s c l r}\right)} e^{-a E_{s c l r}\left(\vec{p}_{s c l r}\right) t}
$$




\subsection{Extracting Matrix Elements From Four-Point Functions}

This section considers the question of how to extract a transition matrix element such as $\left\langle K\left(\vec{p}_{K}\right) \pi\left(\vec{p}_{\pi}\right)|\mathcal{O}| D\left(\vec{p}_{D}\right)\right\rangle$ for the decay $D \rightarrow K \pi$ from four-point correlation functions. This computation applies to $D \rightarrow K \pi$ the arguments previously made for a Euclidean correlation function with a two pion final state ${ }^{30}$. As in that paper, consider the problem first in continuous Euclidean space-time. The finite volume discrete Euclidean space-time lattice result should then be a good approximation to the continuum result provided that $L m_{\pi} \gg 1$ so that the lattice size is much greater than the Compton wavelength of the lightest meson and that $t \delta E \ll 1$ so that integrals are well approximated by sums.

The four-point functions of interest in the decay $D \rightarrow K \pi$ are of the form

$$
\begin{aligned}
& G^{(4)}\left(t_{K}, t_{\pi}, 0, t_{D} ; \vec{p}_{K}, \vec{p}_{\pi}, \vec{p}_{D}\right)= \\
& \quad \int d^{3} \vec{x}_{K} d^{3} \vec{x}_{\pi} d^{3} \vec{x}_{D} e^{\left(\vec{p}_{K} \cdot \vec{x}_{K}+\vec{p}_{\pi} \cdot \vec{x}_{\pi}-\vec{p}_{D} \cdot \vec{x}_{D}\right)} \\
& \quad\left\langle 0\left|\mathrm{~T}\left(\chi_{K}\left(x_{K}\right) \chi_{\pi}\left(x_{\pi}\right) \mathcal{O}(0) \chi_{D}^{\dagger}\left(x_{D}\right)\right)\right| 0\right\rangle
\end{aligned}
$$

where $\chi_{K}, \chi_{\pi}$, and $\chi_{D}$ are interpolating fields for the kaon, pion and $D$ respectively and $\mathcal{O}$ is an effective weak operator. The integrals over the spatial coordinates of the interpolating fields project out specific three-momenta for the initial and final state particles.

Ccnsider the case $t_{K} \gg t_{\pi} \gg 0 \gg t_{D}$. Use the continuum version of con:pleteness to insert sets of single particle states between the pion and kaon fields and between the weak operator $\mathcal{O}$ and the $D$ operator. Then use translational 
invariance to do the integrals over $\vec{x}_{K}$ and $\vec{x}_{D}$. One finds

$$
\begin{aligned}
& G^{(4)}\left(t_{K}, t_{\pi}, 0, t_{D} ; \vec{p}_{K}, \vec{p}_{\pi}, \vec{p}_{D}\right)= \\
& \quad \sum_{A A^{\prime}} \frac{e^{-E_{A}\left(\vec{p}_{K}\right) t_{K}}}{2 E_{A}\left(\vec{p}_{K}\right)} \frac{e^{E_{A^{\prime}}\left(\vec{p}_{D}\right) t_{D}}}{2 E_{A^{\prime}}\left(\vec{p}_{D}\right)} \\
& \quad\left\langle 0\left|\chi_{K}(0)\right| A\left(\vec{p}_{K}\right)\right\rangle\left\langle A^{\prime}\left(\vec{p}_{D}\right)\left|\chi_{D}^{\dagger}(0)\right| 0\right\rangle \\
& \quad \int d^{3} \vec{x}_{\pi} e^{\left(\vec{p}_{\pi} \cdot \vec{x}_{\pi}\right)}\left\langle A\left(\vec{p}_{K}\right)\left|\chi_{\pi}\left(x_{\pi}\right) \mathcal{O}(0)\right| A^{\prime}\left(\vec{p}_{D}\right)\right\rangle .
\end{aligned}
$$

As in the two-point function calculation, states $A$ for which $\left\langle 0\left|\chi_{K}(0)\right| A\left(\vec{p}_{K^{\prime}}\right)\right\rangle \neq 0$ are the kaon and its radial excitations while $\left\langle A^{\prime}\left(\vec{p}_{D}\right)\left|\chi_{D}^{\dagger}(0)\right| 0\right\rangle \neq 0$ for $A^{\prime}$ states that are the $D$ and its radial excitations. Therefore as $t_{K} \rightarrow+\infty$ and $t_{D} \rightarrow-\infty$ the two sums are dominated by the kaon and the $D$ meson respectively. Then, writing $Z_{\vec{K}^{\prime}}\left(\vec{p}_{K}\right)=\left\langle 0\left|\chi_{K}(0)\right| K\left(\vec{p}_{K}\right)\right\rangle$ and $Z_{D}\left(\vec{p}_{D}\right)=\left\langle D\left(\vec{p}_{D}\right)\left|\chi_{D}^{\dagger}(0)\right| 0\right\rangle$ the fourpoint function becomes

$$
\begin{aligned}
& G^{(4)}\left(t_{K}, t_{\pi}, 0, t_{D} ; \vec{p}_{K}, \vec{p}_{\pi}, \vec{p}_{D}\right)= \\
& \quad \frac{e^{-E_{K}\left(\vec{p}_{K}\right) t_{K}}}{2 E_{K}\left(\vec{p}_{K}\right)} \frac{e^{E_{D}\left(\vec{p}_{D}\right) t_{D}}}{2 E_{D}\left(\vec{p}_{D}\right)} Z_{K}\left(\vec{p}_{K}\right) Z_{D}\left(\vec{p}_{D}\right) \\
& \quad \int d^{3} \vec{x}_{\pi} e^{\left(\vec{p}_{\pi} \cdot \vec{x}_{\pi}\right)}\left\langle K\left(\vec{p}_{K}\right)\left|\chi_{\pi}\left(x_{\pi}\right) \mathcal{O}(0)\right| D\left(\vec{p}_{D}\right)\right\rangle .
\end{aligned}
$$

The continuum equivalents of Eq. (4.2.12) the asymptotic form of the pseudoscalar propagator, for the kaon and $D$ are

$$
G_{K}^{(2)}\left(t_{K}, 0 ; \vec{p}_{K}\right) \underset{t_{K} \rightarrow+\infty}{\longrightarrow} \frac{\left|Z_{K}\left(\vec{p}_{K}\right)\right|^{2}}{2 E_{K}\left(\vec{p}_{K}\right)} e^{-E_{K}\left(\vec{p}_{K}\right) t_{K}}
$$

and

$$
G_{D}^{(2)}\left(t_{D}, 0 ; \vec{p}_{D}\right) \underset{t_{D} \rightarrow-\infty}{\longrightarrow} \frac{\left|Z_{D}\left(\vec{p}_{D}\right)\right|^{2}}{2 E_{D}\left(\vec{p}_{D}\right)} e^{E_{D}\left(\vec{p}_{D}\right) t_{D}}
$$

They have exactly the same $t_{K}$ and $t_{D}$ dependence as Eq. (4.4.2). The ratio

$$
F\left(t_{\pi} ; \vec{p}_{K}, \psi_{\pi}, \vec{p}_{D}\right)=Z_{K}\left(\vec{p}_{K}\right) Z_{D}\left(\vec{p}_{D}\right) \frac{G^{(4)}\left(t_{K}, t_{\pi}, 0, t_{D} ; \vec{p}_{K}, \vec{p}_{\pi}, \vec{p}_{D}\right)}{G_{K}^{(2)}\left(t_{K}, 0 ; \vec{p}_{K}\right) G_{D}^{(2)}\left(t_{D}, 0 ; \vec{p}_{D}\right)}
$$


where

$$
\begin{aligned}
F\left(t_{\pi} ; \vec{p}_{K}, \vec{p}_{\pi}, \vec{p}_{D}\right)= & \int d^{3} \vec{x}_{\pi} e^{\left(\vec{p}_{\pi} \cdot \vec{x}_{\pi}\right)} \\
& \left\langle K\left(\vec{p}_{K}\right)\left|\chi_{\pi}\left(x_{\pi}\right) \mathcal{O}(0)\right| D\left(\vec{p}_{D}\right)\right\rangle
\end{aligned}
$$

is independent of both $t_{K}$ and $t_{D}$ as $t_{K} \rightarrow+\infty$ and $t_{D} \rightarrow-\infty$.

Insert a complete set of intermediate states between $\chi_{\pi}$ and $\mathcal{O}$ and use translational invariance to do the integral over $\vec{x}_{\pi}$

$$
\begin{gathered}
F\left(t_{\pi} ; \vec{p}_{K}, \vec{p}_{\pi}, \vec{p}_{D}\right)=\sum_{I}(2 \pi)^{3} \delta^{3}\left(\vec{p}_{K}+\vec{p}_{\pi}-\vec{p}_{I}\right) e^{t_{\pi}\left(E_{K}\left(\vec{p}_{K}\right)-E_{I}\right)} \\
\left\langle K\left(\vec{p}_{K}\right)\left|\chi_{\pi}(0)\right| I, \vec{p}_{I}\right\rangle\left\langle I, \vec{p}_{I}|\mathcal{O}(0)| D\left(\vec{p}_{D}\right)\right\rangle
\end{gathered}
$$

Here the sum over I represents both sums over discrete parameters of the intermediate states and integrals over continuous parameters such as the momenta of the intermediate state collectively represented by $\vec{p}_{I}$. The energy of the intermediate state is $E_{I}$.

In addition to intermediate two particle states containing strongly interacting pions and kaons we must also consider possible contributions from states with other than two particles that couple to the $D$ through the weak operator and that couple to the final $K \pi$ state through strong interactions.

The function $\left\langle K\left(\vec{p}_{K}\right)\left|\chi_{\pi}(0)\right| I, \vec{p}_{I}\right\rangle$ contains both a disconnected piece and a connected pieces. The disconnected piece describes a pion and kaon that do not interact through strong interactions. This disconnected piece leads to a contribution to $F$ of the form

$$
\begin{aligned}
F_{d i s}\left(t_{\pi} ; \vec{p}_{K}, \vec{p}_{\pi}, \vec{p}_{D}\right) & =\frac{\left|Z_{\pi}\left(\vec{p}_{\pi}\right)\right|^{2}}{2 a E_{\pi}\left(\vec{p}_{\pi}\right)} e^{-E_{\pi}\left(\vec{p}_{\pi}\right) t_{\pi}} \\
& \left\langle\boldsymbol{K}\left(\vec{p}_{K}\right) \pi\left(\vec{p}_{\pi}\right) \text { in }|\mathcal{O}(0)| D\left(\vec{p}_{D}\right) i n\right\rangle .
\end{aligned}
$$

Where states are classified as being "in" or "out" states in the sense of the strong interaction scattering matrix. 
The connected pieces of $\left\langle K\left(\vec{p}_{K}\right)\left|\chi_{\pi}(0)\right| I, \vec{p}_{I}\right\rangle$ are related to the strong interaction coupling of intermediate states $\left|I, \vec{p}_{I}\right\rangle$ to a final state consisting of a pion and a kaon. One now defines

$$
\left\langle K\left(\vec{p}_{K}\right)\left|\chi_{\pi}(0)\right| I, \vec{p}_{I}\right\rangle_{c o n} \equiv \frac{\mathcal{M}\left(\vec{p}_{K}, \vec{p}_{\pi} ; I, \vec{p}_{I}\right)}{p_{\pi}^{2}-m_{\pi}^{2}+i \epsilon} Z_{\pi}\left(\vec{p}_{\pi}\right)
$$

where $p_{\pi}=\left(E_{I}-E_{K}\left(\vec{p}_{K}\right), \vec{p}_{\pi}\right)$ with $\vec{p}_{\pi}=\vec{p}_{I}-\vec{p}_{K}$. This definition tells us nothing of the value of the correlation function $\left\langle K\left(\vec{p}_{K}\right)\left|\chi_{\pi}(0)\right| I, \vec{p}_{I}\right\rangle_{\text {con }}$ for values of $E_{I}$ other than when $E_{I}=E_{K}\left(\vec{p}_{K}\right)+E_{\pi}\left(\vec{p}_{\pi}\right)$. For that value of $E_{I}$, LSZ reduction theorem says that the connected correlation function is related to an on-shell transition matrix amplitude

$$
\begin{aligned}
& (2 \pi)^{4} \delta^{4}\left(p_{K}+p_{\pi}-p_{I}\right) i \mathcal{M}\left(\vec{p}_{K}, \vec{p}_{\pi} ; I, \vec{p}_{I}\right)= \\
& \left.\left.\left\langle K\left(\vec{p}_{K}\right) \pi\left(\vec{p}_{\pi}\right) \text { out }\right| I, \vec{p}_{I} \text { in }\right\rangle-\left\langle K\left(\vec{p}_{K}\right) \pi\left(\vec{p}_{\pi}\right) \text { in }\right| I, \vec{p}_{I} \text { in }\right\rangle
\end{aligned}
$$

describing the strong interaction process $I \rightarrow K \pi$. Inserting the definition Eq. (4.4.10) into Eq. (4.4.8) and using the relation

$$
\frac{1}{p_{\pi}^{2}-m_{\pi}^{2}+i \epsilon}=\pi i \frac{\delta\left(p^{0}-E_{\pi}\left(\vec{p}_{\pi}\right)\right)}{2 E_{\pi}\left(\vec{p}_{\pi}\right)}+\mathcal{P} \frac{1}{p_{\pi}^{2}-m_{\pi}^{2}}
$$

to separate out the pole part of the resulting expression yjelds for $t_{\pi} \gg 0$

$$
\begin{aligned}
F\left(t_{\pi} ; \vec{p}_{K}, \vec{p}_{\pi}, \vec{p}_{D}\right)=\frac{Z_{\pi}\left(\vec{p}_{\pi}\right)}{2 E_{\pi}\left(\vec{p}_{\pi}\right)} e^{-E_{\pi}\left(\vec{p}_{\pi}\right) t_{\pi}} \\
\\
{\left[\overline{\mathcal{A}}\left(\vec{p}_{K}, \vec{p}_{\pi}, \vec{p}_{D}\right)+P\left(t_{\pi} ; \vec{p}_{K}, \vec{p}_{\pi}, \vec{p}_{D}\right)\right] . }
\end{aligned}
$$

The common factor multiplying the two terms is the asymptotic pion propagator as in Eq. (4.2.12). The term $\overline{\mathcal{A}}$ is given by

$$
\begin{aligned}
\overrightarrow{\mathcal{A}}=\frac{1}{2}\left\{\left\langleK\left(\vec{p}_{K}\right)\right.\right. & \left.\pi\left(\vec{p}_{\pi}\right) \text { out }|\mathcal{O}(0)| D\left(\vec{p}_{D}\right) \text { in }\right\rangle+ \\
& \left.\left\langle K\left(\vec{p}_{K}\right) \pi\left(\vec{p}_{\pi}\right) \text { in }|\mathcal{O}(0)| D\left(\vec{p}_{D}\right) \text { in }\right\rangle\right\}
\end{aligned}
$$


and is the $D \rightarrow K \pi$ amplitude averaged over "in" and "out" final states. Thus the on-shell amplitude $\overline{\mathcal{A}}$ is real and $\overline{\mathcal{A}}=\cos \delta \mathcal{A}$ where $\delta$ is the strong interaction phase shift and $\mathcal{A}$ is the magnitude of the amplitude.

The second term is given by

$$
\begin{aligned}
& P\left(t_{\pi} ; \vec{p}_{K}, \vec{p}_{\pi}, \vec{p}_{D}\right)= \\
& \mathcal{P} \sum_{I}(2 \pi)^{3} \delta^{3}\left(\vec{p}_{K}+\vec{p}_{\pi}-\vec{p}_{I}\right) e^{-t_{\pi}\left(E_{I}-E_{T}\right)} \\
& \quad \frac{2 E_{\pi}\left(\vec{p}_{\pi}\right) \mathcal{M}\left(\vec{p}_{K}, \vec{p}_{\pi} ; I, \vec{p}_{I}\right)}{\left(E_{I}-E_{T}\right)\left(E_{I}+2 E_{\pi}\left(\vec{p}_{\pi}\right)-E_{T}\right)}\left\langle I, \vec{p}_{I}|\mathcal{O}(0)| D\left(\vec{p}_{D}\right)\right\rangle
\end{aligned}
$$

where $E_{T}=E_{K}\left(\vec{p}_{K}\right)+E_{\pi}\left(\vec{p}_{\pi}\right)$ and the principal value prescription in the integral is indicated by the symbol $\mathcal{P}$.

What about Minkowski space? Then the exponential becomes $e^{i t_{\pi}\left(E_{I}-E_{T}\right)}$. For $t \gg 0$ the exponential, considered a function of $E_{I}$, oscillates wildly canceling the integrand except near the value $E_{T}$. As $t_{\pi}$ tends towards infinity the exponential and the energy denominator can be replaced by $\pi i \delta\left(E_{I}-E_{T}\right) /\left(2 E_{\pi}\left(\vec{p}_{\pi}\right)\right)$. The principal value contribution then combines with the $\overline{\mathcal{A}}$ term of Eq. (4.4.13) to give $\left\langle K\left(\vec{p}_{K}\right) \pi\left(\vec{p}_{\pi}\right)\right.$ out $|\mathcal{O}| D\left(\vec{p}_{D}\right)$ in $\rangle$ as expected.

Return to Euclidean space and Eq. (4.4.15). Suppose that $\vec{p}_{\pi}=-\vec{p}_{K}>\overrightarrow{0}$. Consider the exponential and energy denominators as a function of $E_{I}$. The range of $E_{I}$ is $E_{\min }=m_{K}+m_{\pi} \leq E_{I}<\infty$. The pole giving rise to $\overline{\mathcal{A}}$ is at $E_{I}=E_{T}$. For the region $E_{I}>E_{T}$ with $t_{\pi} \gg 0$ the exponential factor is strongly damped and the principal value contribution will be small. However, for the range $E_{\min } \leq E_{I}<E_{T}$ the exponential is now positive. There will be a large contribution to the principal value integral over this whole range with the largest contribution near $E_{\min }$. The physical meaning of the combination $\overline{\mathcal{A}}+P$ in. Eq. (4.4.13) is unclear since function $P$ depends on $\left\langle K\left(\vec{p}_{K}\right)\left|\chi_{*}(0)\right| I, \vec{p}_{I}\right\rangle_{\text {con }}$ at 
values of $E_{I}$ other than at $E_{T}$ where Eq. (4.4.11) can be used. In particular function $P$ will depend on the choice of interpolating fields used.

Notice that if one were to take $\vec{p}_{\pi}=\vec{p}_{K}=0$ then the biggest contribution to $P$ is for states with $E_{I}=E_{\min }$. But that particular value of $E_{I}$ now corresponds to the position of the pole contribution. Then if $\mathcal{M}$ in Eq. (4.4.15) varies smoothly with $E_{I}$ the off-shell value of $\mathcal{M}$ is approximately the on-shell value and the principal value integral contribution will have physical meaning. The "at rest" condition for correlation functions or amplitudes extracted from the correlation functions is defined to be when we take the three-momenta of external particles to be zero.

Taking the "at rest" condition it is now possible to estimate the principal value contributions in Eq. (4.4.15). Consider an intermediate state consisting of a pion and kaon. This intermediate state should dominate $P$. From Eq. (4.4.15) we now have

$$
\begin{aligned}
& P_{2}\left(t_{\pi} ; \overrightarrow{0}, \overrightarrow{0}, \overrightarrow{0}\right) \approx \\
& \mathcal{P} \int \frac{d^{3} \vec{p}_{K}}{(2 \pi)^{3} 2 E_{K}\left(\vec{p}_{K}\right)} \frac{d^{3} \vec{p}_{\pi}}{(2 \pi)^{3} 2 E_{\pi}\left(\vec{p}_{\pi}\right)}(2 \pi)^{3} \delta^{3}\left(\vec{p}_{K}+\vec{p}_{\pi}\right) \\
& \quad \frac{2 m_{\pi} \mathcal{M}\left(\overrightarrow{0}, \overrightarrow{0} ; \vec{p}_{K}, \vec{p}_{\pi}\right)}{\left(E_{I}-E_{0}\right)\left(E_{I}-E_{0}+2 m_{\pi}\right)} e^{-t_{\pi}\left(E_{I}-E_{\min }\right)} \\
& \quad\left\langle K\left(\vec{p}_{K}\right) \pi\left(\vec{p}_{\pi}\right) \text { in }|\mathcal{O}(0)| D(\overrightarrow{0})\right\rangle .
\end{aligned}
$$

Where $P_{2}$ is the two particle intermediate state piece of $P$. Doing the integral over $\vec{p}_{K}$ and using

$$
d E_{I}=\frac{E_{I}|\vec{p}| d|\vec{p}|}{E_{K}(-\vec{p}) E_{\pi}(\vec{p})}
$$


where $\vec{p}_{\pi}=\vec{p}=-\vec{p}_{K}$ then gives

$$
\begin{aligned}
& P_{2}\left(t_{\pi} ; \overrightarrow{0}, \overrightarrow{0}, \overrightarrow{0}\right) \approx \\
& \frac{1}{2(2 \pi)^{2}} \mathcal{P} \int_{E_{0}} \frac{d \Omega d E_{I}}{4 \pi} e^{-t_{\pi}\left(E_{I}-E_{0}\right)} \\
& \frac{2 m_{\pi}|\vec{p}| \mathcal{M}(\overrightarrow{0}, \overrightarrow{0} ;-\vec{p}, \vec{p})}{E_{I}\left(E_{I}-E_{0}\right)\left(E_{I}-E_{0}+2 m_{\pi}\right)} \\
& \left\langle K\left(\vec{p}_{K}\right) \pi\left(\vec{p}_{\pi}\right) \text { in }|\mathcal{O}(0)| D(\overrightarrow{0})\right\rangle .
\end{aligned}
$$

Making the approximation that the matrix element containing the weak operator and the function $\mathcal{M}$ are smooth functions of $E_{I}$ replace both functions by their values at $E_{I}=E_{0}$. After doing the solid angle integral and expanding in powers of $u=t_{\pi}\left(E_{I}-E_{0}\right)$ one finds

$$
\begin{aligned}
& P_{2}\left(t_{\pi} ; \overrightarrow{0}, \overrightarrow{0}, \overrightarrow{0}\right) \approx\langle K(\overrightarrow{0}) \pi(\overrightarrow{0}) \text { in }|\mathcal{O}(0)| D(\overrightarrow{0})\rangle \\
& \mathcal{M}_{0} \sqrt{\frac{m_{K} m_{\pi}}{2(2 \pi)^{4} E_{0}^{3} t_{\pi}}} \int_{0}^{\infty} \frac{d u}{\sqrt{u}} e^{-u}\left[1+o\left(\frac{u}{t_{\pi}}\right)\right] .
\end{aligned}
$$

The threshold matrix element $\mathcal{M}_{0}$ is related to the s-wave scattering length $\alpha_{K \pi}$ for $K \pi \rightarrow K \pi$ scattering through the relation

$$
-\mathcal{M}_{0}=8 \pi\left(m_{K}+m_{\pi}\right) \alpha_{K \pi}
$$

Doing the integral in Eq. (4.4.18) one then finds

$$
\begin{aligned}
P_{2}\left(t_{\pi} ; \overrightarrow{0}, \overrightarrow{0}, \overrightarrow{0}\right) \approx-\alpha{ }_{K \pi} \sqrt{\frac{4 m_{K} m_{\pi}}{(2 \pi)\left(m_{K}+m_{\pi}\right) t_{\pi}}} \\
\langle K(\overrightarrow{0}) \pi(\overrightarrow{0}) \text { in }|\mathcal{O}(0)| D(\overrightarrow{0})\rangle
\end{aligned}
$$

Next consider potential single particle contributions to Eq. (4.4.15). In this case the integral can be done due to the delta function. The result, non-zero when $E_{\kappa}\left(\vec{p}_{\kappa}\right) \neq E_{T}=E_{K}\left(\vec{p}_{K}\right)+E_{\pi}\left(\vec{p}_{\pi}\right)$, is

$$
\begin{aligned}
& P_{1}\left(t_{\pi} ; \vec{p}_{K}, \vec{p}_{\pi}, \vec{p}_{D}\right)=e^{-t_{\pi}\left(E_{\kappa}\left(\vec{p}_{\kappa}\right)-E_{T}\right)} \\
& \frac{2 E_{\pi}\left(\vec{p}_{\pi}\right) \mathcal{M}\left(\vec{p}_{K}, \vec{p}_{\pi} ; \vec{p}_{\kappa}\right)\left\langle\kappa\left(\vec{p}_{\kappa}\right)|\mathcal{O}(0)| D\left(\vec{p}_{D}\right)\right\rangle}{\left(E_{\kappa}\left(\vec{p}_{\kappa}\right)-E_{T}\right)\left(E_{\kappa}\left(\vec{p}_{\kappa}\right)-E_{T}+2 E_{\pi}\left(\vec{p}_{\pi}\right)\right)}
\end{aligned}
$$


where $\vec{p}_{\kappa}=\vec{p}_{K}+\vec{p}_{\pi}$. The function $\mathcal{M}$ describing the process $\kappa \rightarrow K \pi$ is now evaluated at an off-shell point.

The ratio of the four-point correlation function to the pseudoscalar propagators

$$
\begin{aligned}
R= & Z_{K}\left(\vec{p}_{K}\right) Z_{\pi}\left(\vec{p}_{\pi}\right) Z_{D}\left(\vec{p}_{D}\right) \\
& \frac{G^{(4)}\left(t_{K}, t_{\pi}, 0, t_{D} ; \vec{p}_{K}, \vec{p}_{\pi}, \vec{p}_{D}\right)}{G_{K}^{(2)}\left(t_{K}, 0 ; \vec{p}_{K}\right) G_{\pi}^{(2)}\left(t_{\pi}, 0 ; \vec{p}_{\pi}\right) G_{D}^{(2)}\left(t_{D}, 0 ; \vec{p}_{D}\right)}
\end{aligned}
$$

taken "at rest" where $\vec{p}_{K}=\vec{p}_{\pi}=\vec{p}_{D}=0$ is then

$$
\begin{aligned}
& R= \\
& \langle K(\overrightarrow{0}) \pi(\overrightarrow{0})|\mathcal{O}(0)| D(\overrightarrow{0})\rangle\left[1-\alpha_{K \pi}\left(\frac{2 \mu}{\pi t_{\pi}}\right)^{1 / 2}+\ldots\right]+ \\
& \frac{2 m_{\pi} \mathcal{M}(\kappa \rightarrow K \pi)}{\left(m_{\kappa}-m_{K}\right)^{2}-m_{\pi}^{2}}\langle\kappa(\overrightarrow{0})|\mathcal{O}(0)| D(\overrightarrow{0})\rangle e^{-t_{\pi}\left(m_{\kappa}-m_{K}-m_{\pi}\right)}
\end{aligned}
$$

where $\mu$ is the reduced mass of the pion and the kaon. The $D \rightarrow K \pi$ matrix element is real since it is at threshold.

The scattering length $\alpha_{K \pi}$ can be calculated in chiral perturbation theory ${ }^{31}$. The s-wave scattering length for $\pi K$ states of isospin $1 / 2$ and $3 / 2$ are found to be:

$$
\begin{aligned}
& \alpha_{1 / 2}=2 \frac{\mu}{8 \pi f_{\pi}^{2}}+\operatorname{order}\left(m_{\pi}^{2} \log \left(m_{\pi / \Lambda^{2}}^{2}\right)\right) \\
& \alpha_{3 / 2}=-\frac{\mu}{8 \pi f_{\pi}^{2}}+\operatorname{order}\left(m_{\pi}^{2} \log \left(m_{\pi / \Lambda^{2}}^{2}\right)\right)
\end{aligned}
$$

Where $\mu$ is again the reduced mass and $f_{\pi}$ is the pseudoscalar decay constant. Including one loop effects and coupling to possible resonances, reference 31 finds $\alpha_{1 / 2}=0.19 m_{\pi}^{-1}$ and $\alpha_{3 / 2}=-0.06 m_{\pi}^{-1}$. The chiral $\log$ terms in Eq. (4.4.24) are seen to substantially modify the tree-level chiral perturbation theory prediction that $\alpha_{1 / 2} / \alpha_{3 / 2}=-2$. On the lattice, given sufficiently small statistical errors and a wide enough range in $t$ one can attempt to extract information on the scattering lengths. 
On the lattice it may also be possible to extract the scattering length by studying the volume dependence of the total energy of two-particle $\pi K$ states $^{32}$. Such calculations have been used to calculate the $I=2$ scattering length for two pions ${ }^{33}$.

Lattice weak matrix elements are extracted by forming ratios of four-point correlation functions to two-point functions as in Eq. (4.4.22). The ratio above is commonly referred to as the "time-independent" (TI) ratio. This terminology is fitting for the case of matrix elements with a single hadron in both the initial and final states. In that particular case the $\mathrm{TI}$ ratio is just the matrix element of interest. For the four-point functions considered in $D \rightarrow K \pi$, the term timeindependent ratio is a misnomer since this ratio can still have a residual timedependence from terms due to the manifestations of final state interactions in Euclidean space.

\subsection{Lattice Four-point Functions For $D \rightarrow K \pi$.}

Lattice correlation functions are computed for $D \rightarrow K \pi$ decay modes. "At rest" amplitudes are extracted from four-point correlation functions of the form

$$
\begin{aligned}
& G^{(4)}\left(t_{K}, t_{\pi}, 0, t_{D}\right)= \\
& \quad \sum_{\vec{x}_{K} \vec{x}_{\pi} \vec{x}_{D}}\left\langle 0\left|T\left(\chi_{K}\left(x_{K}\right) \chi_{\pi}\left(x_{\pi}\right) \mathcal{O}(0) \chi_{D}^{\dagger}\left(x_{D}\right)\right)\right| 0\right\rangle
\end{aligned}
$$

These functions are classified according to the topology of the resulting quark lines after we have performed the necessary Wick contractions over quark operators and whether the weak operator is taken to be $\mathcal{O}_{1}$ of Eq. (3.2.9a) or $\mathcal{O}_{2}$ of Eq. (3.2.9b). 
For the mode $D^{0} \rightarrow K^{-} \pi^{+}$we make the following choices for the interpolating fields

$$
\begin{aligned}
& \chi_{D^{0}}\left(x_{D}\right)=-i \overline{\mathrm{u}} \gamma^{5} \mathrm{c}\left(x_{D}\right) \\
& \chi_{K^{-}}\left(x_{K}\right)=-i \overline{\mathrm{u}} \gamma^{5} \mathrm{~s}\left(x_{K}\right) \\
& \chi_{\pi^{+}}\left(x_{\pi}\right)=-i \overline{\mathrm{d}} \gamma^{5} \mathrm{u}\left(x_{\pi}\right)
\end{aligned}
$$

Substituting these operators into Eq. (4.5.1) and writing the operator as $\mathcal{O}_{1}=$ $\overline{\mathrm{s}} \Gamma^{A} \mathrm{c} \overline{\mathrm{u}} \Gamma^{B} \mathrm{~d}$ one has

$$
\begin{array}{r}
G^{(4)}\left(t_{K}, t_{\pi}, 0, t_{D}\right)=i \sum_{\vec{x}_{K} \vec{x}_{\pi} \vec{x}_{D}}\langle 0| T\left(\overline{\mathrm{u}} \gamma^{5} \mathrm{~s}\left(x_{K}\right) \overline{\mathrm{d}} \gamma^{5} \mathrm{u}\left(x_{\pi}\right)\right. \\
\left.\overline{\mathrm{s}} \Gamma^{A} \mathrm{c} \overline{\mathrm{u}} \Gamma^{B} \mathrm{~d}(0) \overline{\mathrm{c}} \gamma^{5} \mathrm{u}\left(x_{D}\right)\right)|0\rangle
\end{array}
$$

The Dirac matrix structure of the effective operator $\mathcal{O}_{1}$ can be symbolized by the notation $\Gamma^{A} \otimes \Gamma^{B}$. The weak operator is written in the form $\Gamma^{A} \otimes \Gamma^{B}$ rather than explicitly in the form $(V-A)^{\mu} \otimes(V-A)^{\mu}$ as it appears in Eq. (3.2.9) since enforcing overall parity conservation will further restrict these operators.

In the $D \rightarrow K \pi$ decay modes a single pseudoscalar $\left(J^{P}=0^{-}\right) \mathrm{D}$ meson decays into two pseudoscalar mesons. The initial state is then parity minus while the final state having zero total angular momentum and two pseudoscalar particles is parity plus. Thus, to conserve parity only the parity changing part of the effective operators is to be considered. This is done by making the replacement

$$
(V-A)^{\mu} \otimes(V-A)^{\mu} \rightarrow \Gamma^{A} \otimes \Gamma^{B}=-V^{\mu} \otimes A^{\mu}-A^{\mu} \otimes V^{\mu}
$$

in the weak operators.

Notice that there are two possible $u$ quark Wick contractions while the Wick contractions are unique for the $c, s$ and $d$ quark fields. Performing the Wick 
contractions one finds

$$
\begin{aligned}
& \text { fig } 8 \pi_{1}\left(t_{K}, t_{\pi}, 0, t_{D}\right)= \\
& i\left\langle\operatorname{Tr}_{s c}\left(\Gamma^{A} \gamma^{5} \sum_{\tilde{x}_{D}} \mathrm{C}^{\dagger}\left(x_{D}, 0 ; \mathcal{U}\right) \sum_{\vec{x}_{K}} \mathrm{U}\left(x_{D}, x_{K} ; \mathcal{U}\right) \gamma^{5} \mathrm{~S}\left(x_{K}, 0 ; \mathcal{U}\right)\right)\right. \\
& \left.\operatorname{Tr}_{s c}\left(\Gamma^{B} \gamma^{5} \sum_{\vec{x}_{\pi}} \mathrm{D}^{\dagger}\left(x_{\pi}, 0 ; \mathcal{U}\right) \mathrm{U}\left(x_{\pi}, 0 ; \mathcal{U}\right)\right)\right\rangle
\end{aligned}
$$

as one Wick contraction and

$$
\begin{aligned}
& \text { fig8an }_{1}\left(t_{K}, t_{\pi}, 0, t_{D}\right)= \\
& i\left\langle-\operatorname{Tr}_{s c}\left(\Gamma^{A} \gamma^{5} \sum_{\vec{x}_{D}} \mathrm{C}^{\dagger}\left(x_{D}, 0 ; \mathcal{U}\right) \mathrm{U}\left(x_{D}, 0 ; \mathcal{U}\right)\right.\right. \\
& \left.\left.\Gamma^{B} \gamma^{5} \sum_{\vec{x}_{\pi}} \mathrm{D}^{\dagger}\left(x_{\pi}, 0 ; \mathcal{U}\right) \sum_{\vec{x}_{K}} \mathrm{U}\left(x_{\pi}, x_{K} ; \mathcal{U}\right) \gamma^{5} \mathrm{~S}\left(x_{K}, 0 ; \mathcal{U}\right)\right)\right\rangle
\end{aligned}
$$

as the other Wick contraction. The traces are over both the spin and color indices of the matrices.

As in the calculation of the propagator correlation functions we recognize the following two products of quark propagators summed over spatial coordinates

$$
\begin{aligned}
& \sum_{\vec{x}_{\pi}} \mathrm{D}^{\dagger}\left(x_{\pi}, 0 ; \mathcal{U}\right) \mathrm{U}\left(x_{\pi}, 0 ; \mathcal{U}\right) \\
& \sum_{\vec{x}_{D}} \mathrm{C}^{\dagger}\left(x_{D}, 0 ; \mathcal{U}\right) \mathrm{U}\left(x_{D}, 0 ; \mathcal{U}\right)
\end{aligned}
$$

The Hermiticity property of quark propagators Eq. (2.6.4) was once again used to write the expression in terms of quark propagators that start from the origin of coordinates at the center of the lattice and end at an arbitrary space-time point. In the intermediate step step called "condensing" these products are precomputed and stored for later use in the weak matrix element calculations. 
Define the quantity

$$
\mathrm{U}_{\mathbf{s}}\left(x, 0 ; t^{\prime}, \mathcal{U}\right) \equiv \sum_{\vec{x}^{\prime}} \mathrm{U}\left(x, x^{\prime} ; \mathcal{U}\right) \gamma^{5} \mathrm{~S}\left(x^{\prime}, 0 ; \mathcal{U}\right)
$$

that appears in Eq. (4.5.5) and Eq. (4.5.6). The $u$ quark propagator that appears here does not begin nor end at the origin. On an $L^{3} \times N$ lattice the calculation of the quark propagator from arbitrary space-time point to arbitrary space-time point would be $L^{3} N$ more expensive than a calculation with the source point restricted to the origin! Fortunately, such a general calculation is not necessary.

Quark propagators are found by solving the linear algebra problem

$$
\sum_{x_{2}} \mathrm{Q}^{-1}\left(x_{1}, x_{2} ; \mathcal{U}\right) \phi\left(x_{2} ; \mathcal{U}\right)=\omega\left(x_{1} ; \mathcal{U}\right)
$$

where $Q^{-1}$ is the inverse propagator matrix for quarks Eq. (2.5.4) and the sum runs over coordinates as well as spin and color indices.

The choice $\omega\left(x_{1} ; \mathcal{U}\right)=\delta_{\vec{x}_{1}, 0} \delta_{t_{1}, 0} \delta_{s_{1}, s} \delta_{c_{1}, c}$ selects the origin of coordinates and spin $s$ and color $c$. Solving Eq. (4.5.9) individually with this $\omega$ for all possible choices of $s$ and $c$ then gives

$$
\phi(x ; \mathcal{U})=\mathrm{Q}(x, 0 ; \mathcal{U})
$$

i.e. the quark propagator matrix from the origin to an arbitrary space-time point. Now select $\omega\left(x_{1} ; \mathcal{U}\right)=\gamma^{5} \mathrm{Q}_{1}\left(x_{1}, 0 ; \mathcal{U}\right) \delta_{t, t^{\prime}}$ which is known as a result of a previous sequence of solutions to Eq. (4.5.9) with a delta function source. The solution to Eq. (4.5.9) is now

$$
\phi\left(x ; t^{\prime}, \mathcal{U}\right)=\sum_{\vec{x}_{1}} \mathrm{Q}_{2}\left(x, x^{\prime} ; \mathcal{U}\right) \gamma^{5} \mathrm{Q}_{1}\left(x^{\prime}, 0 ; \mathcal{U}\right)
$$


This is exactly the same form as the quantity in Eq. (4.5.8). These quantities are called "exponentiated ${ }^{34}$ " or sequential source ${ }^{35}$ quark propagators. In field theoretical language the above process can be described by exponentiating an effective operator to introduce it into the action. In the case corresponding to Eq. (4.5.11) the effective interaction so introduced changes a quark of flayor 1 into a quark of flavor 2 at the location of the $\gamma^{5}$ vertex. The numerical realization of exponentiation is of great practical benefit since quantities such as Eq. (4.5.8) can be computed in just two matrix inversion steps.

Once exponentiated quark propagators are computed they are used in the quark propagator condensing just like the normal quark propagators. Thus the quantities

$$
\begin{gathered}
\sum_{\vec{x}_{D}} \mathrm{C}^{\dagger}\left(x_{D}, 0 ; \mathcal{U}\right) \mathrm{U}_{\mathrm{s}}\left(x_{D}, 0 ; t_{K}, \mathcal{U}\right) \\
\sum_{\vec{x}_{\pi}} \mathrm{D}^{\dagger}\left(x_{\pi}, 0 ; \mathcal{U}\right) \mathrm{U}_{\mathbf{s}}\left(x_{\pi}, 0 ; t_{K}, \mathcal{U}\right)
\end{gathered}
$$

are computed and stored in files as an intermediate step in the numerical analysis.

\subsection{1. $\quad D^{0} \rightarrow K^{-} \pi^{+}$Four-point Functions}

Rewriting Eq. (4.5.5) and Eq. (4.5.6) in terms of exponentiated quark propagators then gives for the mode $D^{0} \rightarrow K^{-} \pi^{+}$

$$
\begin{aligned}
\operatorname{fig} 8 \pi_{1}\left(t_{K}, t_{\pi}, 0, t_{D}\right)_{D^{0} \rightarrow K^{-} \pi^{+}}= & \\
& i\left\langle\operatorname{Tr}_{s c}\left(\Gamma^{A} \gamma^{5} \sum_{\vec{x}_{D}} \mathrm{C}^{\dagger}\left(x_{D}, 0 ; \mathcal{U}\right) \mathrm{U}_{\mathbf{s}}\left(x_{D}, 0 ; t_{K}, \mathcal{U}\right)\right)\right. \\
& \left.\operatorname{Tr}_{s c}\left(\Gamma^{B} \gamma^{5} \sum_{\vec{x}_{\pi}} \mathrm{D}^{\dagger}\left(x_{\pi}, 0 ; \mathcal{U}\right) \mathrm{U}\left(x_{\pi}, 0 ; \mathcal{U}\right)\right)\right\rangle
\end{aligned}
$$


and

$$
\begin{aligned}
& \operatorname{fig}_{1} \operatorname{an}_{1}\left(t_{K}, t_{\pi}, 0, t_{D}\right)_{D^{0} \rightarrow K^{-} \pi^{+}}= \\
& i\left\langle-\operatorname{Tr}_{s c}\left(\Gamma^{A} \gamma^{5} \sum_{\vec{x}_{D}} \mathrm{C}^{\dagger}\left(x_{D}, 0 ; \mathcal{U}\right) \mathrm{U}\left(x_{D}, 0 ; \mathcal{U}\right)\right.\right. \\
&\left.\left.\Gamma^{B} \gamma^{5} \sum_{\vec{x}_{\pi}} \Gamma^{\dagger}\left(x_{\pi}, 0 ; \mathcal{U}\right) \mathrm{U}_{\mathrm{s}}\left(x_{\pi}, 0 ; t_{K}, \mathcal{U}\right)\right)\right\rangle
\end{aligned}
$$

Considering the operator $\mathcal{O}_{2}=\overline{\mathrm{s}} \Gamma^{B} \mathrm{~d} \mathrm{u} \Gamma^{A} c$ Wick contractions of Eq. (4.5.3) yield correlation functions

$$
\begin{aligned}
\operatorname{fig}_{8} \pi_{2}\left(t_{K}, t_{\pi}, 0, t_{D}\right)_{D^{0} \rightarrow K^{-} \pi^{+}}= \\
i\left\langle-\operatorname{Tr}_{s c}\left(\Gamma^{A} \gamma^{5} \sum_{\vec{x}_{D}} \mathrm{C}^{\dagger}\left(x_{D}, 0 ; \mathcal{U}\right) \mathrm{U}_{\mathbf{s}}\left(x_{D}, 0 ; t_{K}, \mathcal{U}\right)\right.\right. \\
\left.\left.\quad \Gamma^{B} \gamma^{5} \sum_{\vec{x}_{\pi}} \mathrm{D}^{\dagger}\left(x_{\pi}, 0 ; \mathcal{U}\right) \mathrm{U}\left(x_{\pi}, 0 ; \mathcal{U}\right)\right)\right\rangle
\end{aligned}
$$

and

$$
\begin{aligned}
&{\operatorname{fig} 8 \operatorname{an}_{2}}_{2}\left(t_{K}, t_{\pi}, 0, t_{D}\right)_{D^{0} \rightarrow K^{-} \pi^{+}}= \\
& i\left\langle\operatorname{Tr}_{s c}\left(\Gamma^{1} \gamma^{5} \sum_{\vec{x}_{D}} \mathrm{C}^{\dagger}\left(x_{D}, 0 ; \mathcal{U}\right) \mathrm{U}\left(x_{D}, 0 ; \mathcal{U}\right)\right)\right. \\
&\left.\operatorname{Tr}_{s c}\left(\Gamma^{B} \gamma^{5} \sum_{\vec{x}_{\pi}} \mathrm{D}^{\dagger}\left(x_{\pi}, 0 ; \mathcal{U}\right) \mathrm{U}_{\mathbf{s}}\left(x_{\pi}, 0 ; t_{K}, \mathcal{U}\right)\right)\right\rangle
\end{aligned}
$$

The four-point correlation functions need to calculate the amplitude $\mathcal{A}\left(D^{0} \rightarrow\right.$ $K^{-} \pi^{+}$) are then fig8 $8 \pi_{1}$, fig8 $8 \pi_{2}$, fig8an 1 , and fig8an ${ }_{2}$.

\subsection{2. $D^{+} \rightarrow \bar{K}^{0} \pi^{+}$Four-point Functions}

The four-point correlation function Eq. (4.5.3) for the mode $D^{+} \rightarrow \bar{K}^{0} \pi^{+}$with interpolating fields

$$
\begin{aligned}
& \chi_{D^{+}}\left(x_{D}\right)=-i \overline{\mathrm{d}} \gamma^{5} \mathrm{c}\left(x_{D}\right) \\
& \chi_{K^{0}}\left(x_{K}\right)=-i \overline{\mathrm{d}} \gamma^{5} \mathrm{~s}\left(x_{K}\right) \\
& \chi_{\pi^{+}}\left(x_{\pi}\right)=-i \overline{\mathrm{d}} \gamma^{5} \mathrm{u}\left(x_{\pi}\right)
\end{aligned}
$$


and operator $\mathcal{O}_{1}$ leads to the expressions

$$
\begin{aligned}
\text { fig8 } 8 \pi_{1}\left(t_{K}, t_{\pi}, 0, t_{D}\right)_{D^{+} \rightarrow K^{0} \pi^{+}}= \\
\qquad i\left\langle\operatorname{Tr}_{s c}\left(\Gamma^{A} \gamma^{5} \sum_{\vec{x}_{D}} \mathrm{C}^{\dagger}\left(x_{D}, 0 ; \mathcal{U}\right) \mathrm{D}_{\mathrm{s}}\left(x_{D}, 0 ; t_{K}, \mathcal{U}\right)\right)\right. \\
\left.\quad \operatorname{Tr}_{s c}\left(\Gamma^{B} \gamma^{5} \sum_{\vec{x}_{\pi}} \mathrm{D}^{\dagger}\left(x_{\pi}, 0 ; \mathcal{U}\right) \mathrm{U}\left(x_{\pi}, 0 ; \mathcal{U}\right)\right)\right\rangle
\end{aligned}
$$

and

$$
\begin{aligned}
{\text { fig } 8 \mathrm{~K}_{1}}_{1}\left(t_{K}, t_{\pi}, 0, t_{D}\right)_{D^{+} \rightarrow K^{0} \pi^{+}}= & \\
& i\left\langle-\operatorname{Tr}_{s c}\left(\Gamma^{A} \gamma^{5} \sum_{\vec{x}_{D}} \mathrm{C}^{\dagger}\left(x_{D}, 0 ; \mathcal{U}\right) \mathrm{D}_{\mathrm{u}}\left(x_{D}, 0 ; t_{\pi}, \mathcal{U}\right)\right.\right. \\
& \left.\left.\Gamma^{B} \gamma^{5} \sum_{\vec{x}_{K}} \mathrm{D}^{\dagger}\left(x_{K}, 0 ; \mathcal{U}\right) \mathrm{S}\left(x_{K}, 0 ; \mathcal{U}\right)\right)\right\rangle
\end{aligned}
$$

While taking the operator to be $\mathcal{O}_{2}$ gives

$$
\begin{aligned}
& \text { fig8 } 8 \pi_{2}\left(t_{K}, t_{\pi}, 0, t_{D}\right)_{D^{+} \rightarrow K^{0} \pi^{+}}= \\
& i\left\langle-\operatorname{Tr}_{s c}\left(\Gamma^{A} \gamma^{5} \sum_{\vec{x}_{D}} \mathrm{C}^{\dagger}\left(x_{D}, 0 ; \mathcal{U}\right) \mathrm{D}_{\mathrm{s}}\left(x_{D}, 0 ; t_{K}, \mathcal{U}\right)\right.\right. \\
& \left.\left.\quad \Gamma^{B} \gamma^{5} \sum_{\vec{x}_{\pi}} \mathrm{D}^{\dagger}\left(x_{\pi}, 0 ; \mathcal{U}\right) \mathrm{U}\left(x_{\pi}, 0 ; \mathcal{U}\right)\right)\right\rangle
\end{aligned}
$$

and

$$
\begin{aligned}
\operatorname{fig}_{2} 8 \mathrm{~K}_{2}\left(t_{K}, t_{\pi}, 0, t_{D}\right)_{D^{+} \rightarrow K^{0} \pi^{+}}= & \\
& i\left\langle\operatorname{Tr}_{s c}\left(\Gamma^{A} \gamma^{5} \sum_{\vec{x}_{D}} \mathrm{C}^{\dagger}\left(x_{D}, 0 ; \mathcal{U}\right) \mathrm{D}_{\mathrm{u}}\left(x_{D}, 0 ; t_{\pi}, \mathcal{U}\right)\right)\right. \\
& \left.\operatorname{Tr}_{s c}\left(\Gamma^{B} \gamma^{5} \sum_{\vec{x}_{K}} \mathrm{D}^{\dagger}\left(x_{K}, 0 ; \mathcal{U}\right) \mathrm{S}\left(x_{K}, 0 ; \mathcal{U}\right)\right)\right\rangle
\end{aligned}
$$


Notice that both of the modes $D^{0} \rightarrow K^{-} \pi^{+}$and $D^{+} \rightarrow \bar{K}^{0} \pi^{+}$contain correlation functions classified as fig8 8 . Comparing Eq. (4.5.13) and Eq. (4.5.18) fig8 $\pi_{1}$ correlation functions for the two modes, the expressions differ by the replacement of the $\mathrm{u}$ quark by a $\mathrm{d}$ quark. Since all numerical simulations are performed with degenerate $\mathrm{u}$ and $\mathrm{d}$ masses, the two functions are equal. Using $m_{\mathrm{u}}=m_{\mathrm{d}}$ is expected to be a good approximation since although $m_{\mathrm{d}} \approx 2 m_{\mathrm{u}}$ for the physical current quark masses, they are both small relative to $\Lambda_{Q C D}$. The result is that isospin symmetry breaking effects are small. On the lattice isospin symmetry is made exact.

\subsection{3. $\quad D^{0} \rightarrow \bar{K}^{0} \pi^{0}$ Four-point Functions}

For the mode $D^{0} \rightarrow \bar{K}^{0} \pi^{0}$ the $D^{0}$ interpolating field is the same as in Eq. (4.5.2) and the $\bar{K}^{0}$ interpolating field is given in Eq. (4.5.17). The $\pi^{0}$ interpolating field is then taken to be

$$
\chi_{\pi^{0}}\left(x_{\pi}\right)=\frac{-i}{\sqrt{2}}\left(\overline{\mathrm{u}} \gamma^{5} \mathrm{u}-\overline{\mathrm{d}} \gamma^{5} \mathrm{~d}\right)\left(x_{\pi}\right)
$$

Starting with Eq. (4.5.3) and performing the Wick contractions for $\mathcal{O}_{1}$ gives

$$
\begin{aligned}
& {\text { fig } 8 K_{1}}\left(t_{K}, t_{\pi}, 0, t_{D}\right)_{D^{0} \rightarrow K^{0} \pi^{0}}= \\
& \frac{i}{\sqrt{2}}\left\langle-\operatorname{Tr}_{s c}\left(\Gamma^{A} \gamma^{5} \sum_{\vec{x}_{D}} \mathrm{C}^{\dagger}\left(x_{D}, 0 ; \mathcal{U}\right) \mathrm{U}_{\mathrm{u}}\left(x_{D}, 0 ; t_{\pi}, \mathcal{U}\right)\right.\right. \\
& \left.\left.\Gamma^{B} \gamma^{5} \sum_{\vec{x}_{K}} \mathrm{D}^{\dagger}\left(x_{K}, 0 ; \mathcal{U}\right) \mathrm{S}\left(x_{K}, 0 ; \mathcal{U}\right)\right)\right\rangle \\
& {\operatorname{fig} 8 \operatorname{an}_{1}}\left(t_{K}, t_{\pi}, 0, t_{D}\right)_{D^{0} \rightarrow \bar{K}^{0} \pi^{0}}= \\
& -\frac{i}{\sqrt{2}}\left\langle-\operatorname{Tr}_{s c}\left(\Gamma^{A} \gamma^{5} \sum_{\vec{x}_{D}} \mathrm{C}^{\dagger}\left(x_{D}, 0 ; \mathcal{U}\right) \mathrm{U}\left(x_{D}, 0 ; \mathcal{U}\right)\right.\right. \\
& \left.\left.\Gamma^{B} \gamma^{5} \sum_{\vec{x}_{\pi}} \mathrm{D}^{\dagger}\left(x_{\pi}, 0 ; \mathcal{U}\right) \mathrm{D}_{\mathrm{s}}\left(x_{\pi}, 0 ; t_{K}, \mathcal{U}\right)\right)\right\rangle
\end{aligned}
$$




$$
\begin{gathered}
{\operatorname{fig} 8 \operatorname{dis}_{1}\left(t_{K}, t_{\pi}, 0, t_{D}\right)_{D^{0} \rightarrow K^{0} \pi^{0}}=}_{\frac{i}{\sqrt{2}}}\left\langle\operatorname{Tr}_{s c}\left(\gamma^{5} \sum_{\vec{x}_{\pi}}\left(\mathrm{U}\left(x_{\pi}, x_{\pi} ; \mathcal{U}\right)-\mathrm{D}\left(x_{\pi}, x_{\pi} ; \mathcal{U}\right)\right)\right)\right. \\
\operatorname{Tr}_{s c}\left(\Gamma^{A} \gamma^{5} \sum_{\vec{x}_{D}} \mathrm{C}^{\dagger}\left(x_{D}, 0 ; \mathcal{U}\right) \mathrm{U}\left(x_{D}, 0 ; \mathcal{U}\right)\right. \\
\left.\left.\Gamma^{B} \gamma^{5} \sum_{\vec{x}_{K}} \mathrm{D}^{\dagger}\left(x_{K}, 0 ; \mathcal{U}\right) \mathrm{S}\left(x_{K}, 0 ; \mathcal{U}\right)\right)\right\rangle .
\end{gathered}
$$

The correlation functions for $\mathcal{O}_{2}$ are

$$
\begin{aligned}
& \operatorname{fig} 8 \mathrm{~K}_{2}\left({ }^{+}{ }_{K}, t_{\pi}, 0, t_{D}\right)_{D^{0} \rightarrow K^{0} \pi^{0}}= \\
& \frac{i}{\sqrt{2}}\left\langle\operatorname{Tr}_{s c}\left(\Gamma^{A} \gamma^{5} \sum_{\vec{x}_{D}} \mathrm{C}^{\dagger}\left(x_{D}, 0 ; \mathcal{U}\right) \mathrm{U}_{\mathbf{u}}\left(x_{D}, 0 ; t_{n}, \mathcal{U}\right)\right)\right. \\
& \left.\operatorname{Tr}_{s c}\left(\Gamma^{B} \gamma^{5} \sum_{\vec{x}_{K}} D^{\dagger}\left(x_{K}, 0 ; \mathcal{U}\right) \mathrm{S}\left(x_{K}, 0 ; \mathcal{U}\right)\right)\right\rangle
\end{aligned}
$$

$$
\begin{aligned}
& \text { fig8an }_{2}\left(t_{K}, t_{\pi}, 0, t_{D}\right)_{D^{0} \rightarrow K^{0} \pi^{0}}= \\
& -\frac{i}{\sqrt{2}}\left\langle\operatorname{Tr}_{s c}\left(\Gamma^{A} \gamma^{5} \sum_{\vec{x}_{D}} \mathrm{C}^{\dagger}\left(x_{D}, 0 ; \mathcal{U}\right) \mathrm{U}\left(x_{D}, 0 ; \mathcal{U}\right)\right)\right. \\
& \left.\quad \operatorname{Tr}_{s c}\left(\Gamma^{B} \gamma^{5} \sum_{\vec{x}_{\pi}} D^{\dagger}\left(x_{\pi}, 0 ; \mathcal{U}\right) D_{\mathrm{s}}\left(x_{\pi}, 0 ; t_{K}, \mathcal{U}\right)\right)\right\rangle
\end{aligned}
$$

$\operatorname{fig} \operatorname{dis}_{2}\left(t_{K}, t_{\pi}, 0, t_{D}\right)_{D^{0} \rightarrow K^{0} x^{0}}=$

$$
\begin{gathered}
\frac{i}{\sqrt{2}}\left\langle-\operatorname{Tr}_{s c}\left(\gamma^{5} \sum_{\vec{x}_{\pi}}\left(\mathrm{U}\left(x_{\pi}, x_{\pi} ; \mathcal{U}\right)-\mathrm{D}\left(r_{-}, x_{\pi} ; \mathcal{U}\right)\right)\right)\right. \\
\operatorname{Tr}_{s c}\left(\Gamma^{A} \gamma^{5} \sum_{\vec{x}_{D}} \mathrm{C}^{\dagger}\left(x_{D}, 0 ; \mathcal{U}\right) \mathrm{U}\left(x_{D}, 0 ; \mathcal{U}\right)\right) \\
\left.\operatorname{Tr}_{s c}\left(\Gamma^{B} \gamma^{5} \sum_{\vec{x}_{K}} D^{\dagger}\left(x_{K}, 0 ; \mathcal{U}\right) \mathrm{S}\left(x_{K}, 0 ; \mathcal{U}\right)\right)\right\rangle .
\end{gathered}
$$


These functions are computed in the limit of exact isospin invariance where $m_{\mathbf{u}}=m_{\mathrm{d}}$. In this limit the fig8dis functions of Eq. (4.5.25) and Eq. (4.5.28) are zero due to the cancellation of the $u$ and $d$ quark loops. Note that in the degenerate $u$ and $d$ limit

$$
\begin{aligned}
\text { fig8K }_{D^{0} \rightarrow K^{0} \pi^{0}} & =\frac{1}{\sqrt{2}} \text { fig8 }_{D^{0} \rightarrow K^{-} \pi^{+}} \\
\text {fig8an }_{D^{0} \rightarrow K^{0} \pi^{0}} & =-\frac{1}{\sqrt{2}} \text { fig8an }_{D^{0} \rightarrow K^{-} \pi^{+}}
\end{aligned}
$$

The factor of $\frac{1}{\sqrt{2}}$ arises from the Clebsch-Gordon coefficient in the definition of the $\pi^{0}$ interpolating field Eq. (4.5.22). The relative minus sign between the ūu and $\overline{\mathrm{d} d}$ terms in $\chi_{\pi^{0}}$ is also responsible for the relative minus sign between fig8an functions since for $D^{0} \rightarrow \bar{K}^{0} \pi^{0}$ only the $\bar{d} d$ term contributes.

\subsection{4. $D \rightarrow K \pi$ Lattice Diagrams and Large $N$}

With the $u$ and $d$ quarks degenerate there are only six four-point correlation functions that need to be calculated. These four-point correlation functions and the corresponding defining equations are:

$$
\begin{array}{ll}
\text { fig8 } 8 \pi_{1} & \text { Eq. (4.5.13) } \\
\text { fig8 } 8 \pi_{2} & \text { Eq. (4.5.15) } \\
\text { fig8an }_{1} & \text { Eq. (4.5.14) } \\
\text { fig8an }_{2} & \text { Eq. (4.5.16) } \\
\text { fig8K }_{1} & \text { Eq. (4.5.19) } \\
\text { fig8K }_{2} & \text { Eq. (4.5.21). }
\end{array}
$$

For each of these correlation functions there is an associated diagram as shown in Fig. 4.5.1. In each of these diagrams the effective weak operator is represented by the open circles joined by a dashed line. The dashed lines in $\mathcal{O}_{1}$ type diagrams 

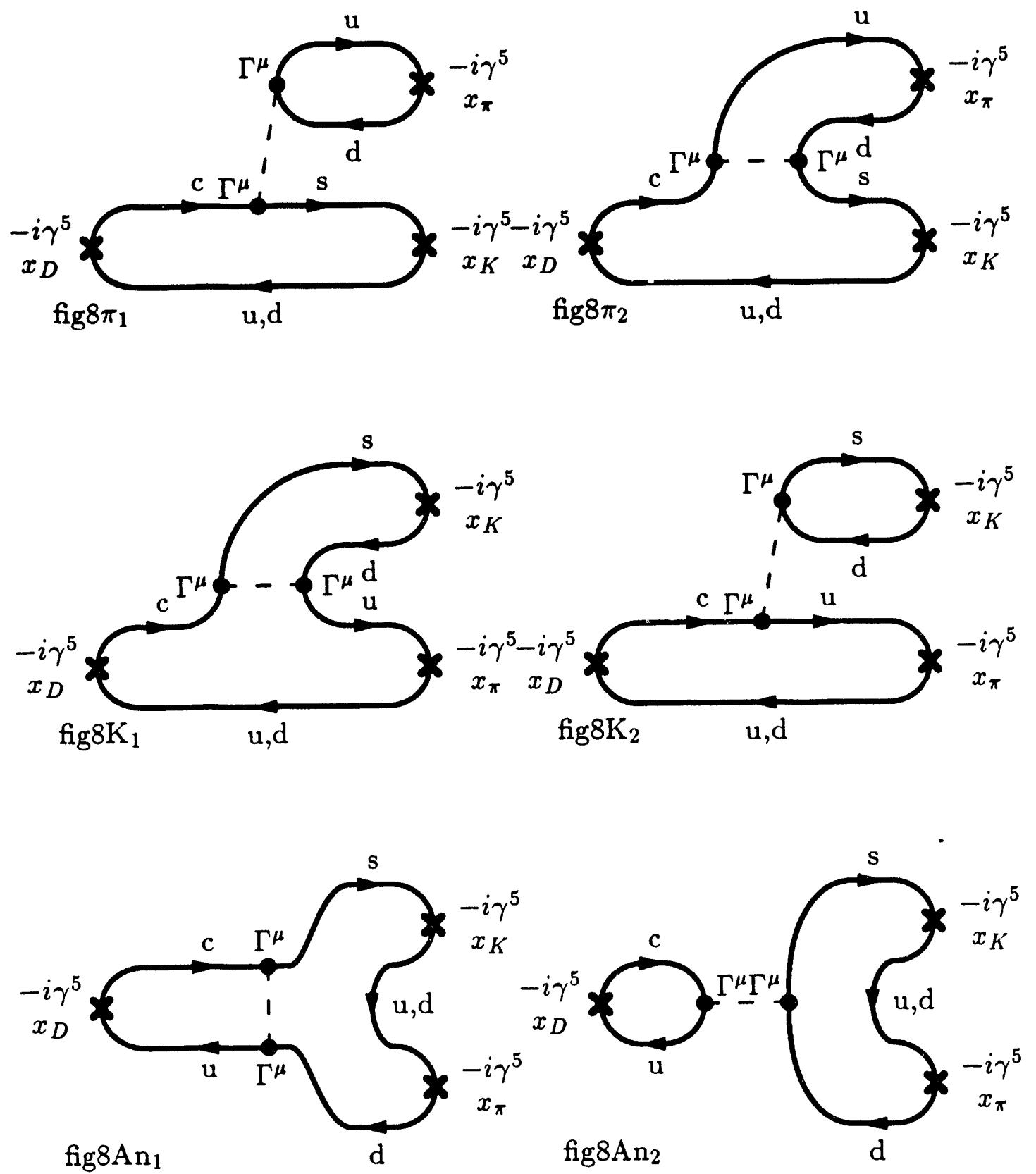

Figure 4.5.1: Graphical representation of the fig8 correlation functions for $D \rightarrow K \pi$ decay modes. In each diagram the dots connected by dashed line represent the effective weak operator located at the space-time origin. A cross represents the location of an $-i \gamma^{5}$ insertion for a pseudoscalar meson.

correspond to an effective charged current exchange while these lines in $\mathcal{O}_{2}$ type 
diagrams are an effective neutral current exchange that results from the effective weak operator formalism.

The solid lines represent quark propagators. Each quark loop in these diagrams corresponds to a trace over a product of quark propagators and Dirac matrices as in the corresponding definition of the given correlation function. Each of these traces contributes a factor of -1 to the overall phase of the function. The crosses in these diagrams represent the locations of the field operators for the mesons. For pseudoscalar particles the crosses represent the insertion of $a-i \gamma^{5}$ matrix at that point in the product of propagators around the loop.

One can see an immediate correspondence between these lattice diagrams and the quark level Feynman diagrams one would draw for the continuum. Hence, fig $8 \pi$ is related to the continuum "spectator" diagram, while fig $8 \mathrm{~K}$ is related to the "color suppressed spectator" diagram, and fig8an is related to the "weak annihilation" diagram. It is extremely important to note that the correspondence between lattice diagrams and continuum Feynman diagrams is not as direct as it may appear.

These lattice diagrams, evaluated in quenched lattice $\mathrm{QCD}$, are the first term in an expansion in the number of quark loops which include effects to all orders in the strong interactions. Including such non-perturbative effects in such a natural way within the language of continuum diagrams can create a great deal of confusion especially when one considers final state interactions. The problem is that if one starts with a continuum Feynman diagram and then includes final state interactions by dressing the diagrams with soft gluons or by considering the Zweig suppressed annihilation of final state $\overline{\mathrm{q}} \mathrm{q}$ pairs, the resulting diagrams can describe contributions within the lattice diagram picture to lattice diagrams, 
other than the one which most directly seems to correspond to the continuum Feynman diagram. The lattice diagrams correspond to the continuum diagrams in the absence of QCD. When QCD is turned on and one attempts to draw Feynman diagrams including the exchange of gluons the definition of diagrams must be considered carefully.

A useful approach to the classification of the continuum diagrams is the $1 / \mathrm{N}$ expansion $^{5}$ where $\mathrm{N}$ is the number of colors. The $1 / \mathrm{N}$ approach is also useful for lattice diagrams. With the counting rules for the large $\mathrm{N}$ expansion the latiice diagrams can be classified according to their leading order $\mathrm{N}$ behavior. These rules are:

1. Include a factor $\mathrm{N}$ for each closed quark loop.

2. Include a factor $\frac{1}{\sqrt{N}}$ for each quark-meson vertex (crosses in Fig. 4.5.1).

3. Include a factor of $\frac{1}{\sqrt{N}}$ for each quark-gluon vertex (only for soft gluons).

The four-point functions are written as an expansion in $1 / \mathrm{N}$

$$
G^{(4)}=\sqrt{N}\left[f_{0}+\frac{1}{N} f_{1}+\ldots\right]
$$

Where by convention the prefactor of $\sqrt{N}$ has been factored out of each term. The counting rules then give the values shown in Table 4.5.1 for the leading order contributions of the four-point functions.

\subsubsection{Lattice Vacuum Saturation Functions}

Factorization and the vacuum saturation approximation are frequently used in continuum models. The validity of this approximation can be tested on the lattice. This test of vacuum saturation requires the calculation of new two-point and 


\begin{tabular}{|c|c|c|}
\hline $\begin{array}{c}\text { diagram } \\
\text { description }\end{array}$ & $\begin{array}{c}\text { correlation } \\
\text { function }\end{array}$ & $\begin{array}{c}\text { leading } \mathrm{N} \\
\text { order }\end{array}$ \\
\hline spectator & $\begin{array}{c}\text { fig8 } 8 \pi_{1} \\
\text { fig8 } 8 \pi_{2}\end{array}$ & $\frac{1}{N}$ \\
\hline $\begin{array}{c}\text { color suppressed } \\
\text { spectator }\end{array}$ & $\begin{array}{c}\text { fig8 } \\
\text { fig8 }\end{array}$ & $\frac{1}{N}$ \\
\hline weak & fig8an $_{1}$ & $\frac{1}{N}$ \\
annihilation & fig8an $_{2}$ & $\frac{1}{N}$ \\
\hline
\end{tabular}

Table 4.5.1: Leading order large $\mathrm{N}$ behavior of fig8 diagrams

three-point correlation functions in addition to the four-point functions and propagator functions already discussed. In this section these extra diagrams will be enumerated. Questions of renormalization and how to relate these computations to the continuum are postponed until section 4.8 .

Consider the correlation function

$$
\sum_{\vec{x}_{K} \vec{x}_{\pi} \vec{x}_{D}}\left\langle 0\left|\mathrm{~T}\left(\chi_{K}\left(x_{K}\right) \chi_{\pi}\left(x_{\pi}\right) \mathcal{O}(0) \chi_{D}^{\dagger}\left(x_{D}\right)\right)\right| 0\right\rangle
$$

where the operator $\mathcal{O}$ is $\mathcal{O}_{1}$ or $\mathcal{O}_{2}$ of Eq. (3.2.9a). Inserting the vacuum state every possible way yields non-zero contributions for:

$$
\begin{aligned}
\text { fig8 } 8 \pi_{1}^{V S}\left(t_{K}, t_{\pi}, 0, t_{D}\right)= & \sum_{\vec{x}_{\pi}}\left\langle 0\left|\chi_{\pi}\left(x_{\pi}\right) \overline{\mathrm{u}}^{a} \Gamma^{A} \mathrm{~d}^{a}(0)\right| 0\right\rangle \\
& \sum_{\vec{x}_{K} \vec{x}_{D}}\left\langle 0\left|\chi_{K}\left(x_{K}\right) \overline{\mathrm{s}}^{b} \Gamma^{B} \mathrm{c}^{b}(0) \chi_{D}^{\dagger}\left(x_{D}\right)\right| 0\right\rangle \\
\text { fig8 } 8 \pi_{2}^{V S}\left(t_{K}, t_{\pi}, 0, t_{D}\right)= & \sum_{\vec{x}_{\pi}}\left\langle 0\left|\chi_{\pi}\left(x_{\pi}\right) \overline{\mathrm{u}}^{a} \Gamma^{A} \mathrm{~d}^{b}(0)\right| 0\right\rangle \\
& \sum_{\vec{x}_{K} \vec{x}_{D}}\left\langle 0\left|\chi_{K}\left(x_{K}\right) \overline{\mathrm{s}}^{b} \Gamma^{B} \mathrm{c}^{a}(0) \chi_{D}^{\dagger}\left(x_{D}\right)\right| 0\right\rangle
\end{aligned}
$$




$$
\begin{aligned}
& \operatorname{fig}_{\mathrm{f}} \mathrm{K}_{1}^{V S}\left(t_{K}, t_{\pi}, 0, t_{D}\right)=\sum_{\overrightarrow{\boldsymbol{x}}_{K}}\left\langle 0\left|\chi_{K}\left(x_{K}\right) \overline{\mathrm{s}}^{a} \Gamma^{A} \mathrm{~d}^{b}(0)\right| 0\right\rangle \\
& \sum_{\vec{x}_{\pi} \vec{x}_{D}}\left\langle 0\left|\chi_{\pi}\left(x_{\pi}\right) \overline{\mathrm{u}}^{b} \Gamma^{B} \mathrm{c}^{a}(0) \chi_{D}^{\dagger}\left(x_{D}\right)\right| 0\right\rangle
\end{aligned}
$$

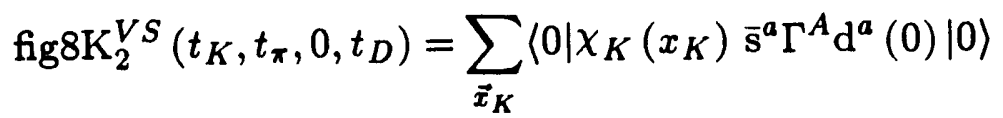

$$
\begin{aligned}
& \sum_{\vec{x}_{\pi} \vec{x}_{D}}\left\langle 0\left|\chi_{\pi}\left(x_{\pi}\right) \overline{\mathrm{u}}^{b} \Gamma^{B} \mathrm{c}^{b}(0) \chi_{D}^{\dagger}\left(x_{D}\right)\right| 0\right\rangle \\
& \operatorname{fig}_{1}^{V S}\left(t_{K}, t_{\pi}, 0, t_{D}\right)=\sum_{\vec{x}_{D}}\left\langle 0\left|\overline{\mathrm{u}}^{a} \Gamma^{A} \mathrm{c}^{b}(0) \chi_{D}^{\dagger}\left(x_{D}\right)\right| 0\right\rangle \\
& \sum_{\overrightarrow{\boldsymbol{x}}_{K} \overrightarrow{\boldsymbol{x}}_{\boldsymbol{\pi}}}\left\langle 0\left|\chi_{K}\left(x_{K}\right) \chi_{\pi}\left(x_{\pi}\right) \overline{\mathrm{s}}^{b} \Gamma^{B} \mathrm{~d}^{a}(0)\right| 0\right\rangle
\end{aligned}
$$

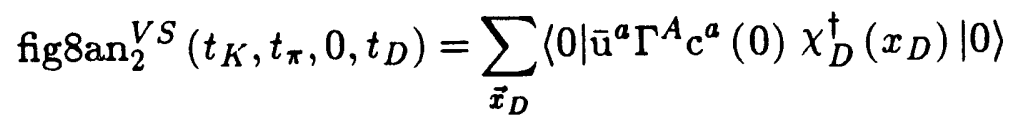

$$
\begin{aligned}
& \sum_{\vec{x}_{K} \vec{x}_{\pi}}\left\langle 0\left|\chi_{K}\left(x_{K}\right) \chi_{\pi}\left(x_{\pi}\right) \bar{s}^{b} \Gamma^{B} \mathrm{~d}^{b}(0)\right| 0\right\rangle
\end{aligned}
$$

where $\Gamma^{A}=\Gamma^{B}=(V-A)^{\mu}$.

In Eq. (4.5.32), Eq. (4.5.33) and Eq. (4.5.35; the Fierz identity

$$
(V-A)_{\alpha \beta}^{\mu} \otimes(V-A)_{\omega \tau}^{\mu}=-(V-A)_{\alpha \tau}^{\mu} \otimes(V-A)_{\omega \beta}^{\mu}
$$

has been used to interchange the quark fields within operators $\mathcal{O}_{1}$ or $\mathcal{O}_{2}$ when necessary before vacuum saturating the correlation function. Hence, in Eq. (4.5.32) for example,

$$
\mathcal{O}_{2}=\overline{\mathrm{s}}^{a}(V-A)^{\mu} \mathrm{d}^{a} \overline{\mathrm{u}}^{b}(V-A)^{\mu} \mathrm{c}^{b}
$$

is Fierz transformed into

$$
\mathcal{O}_{2}=\overline{\mathrm{s}}^{a}(V-A)^{\mu} \mathrm{c}^{b} \overline{\mathrm{u}}^{b}(V-A)^{\mu} \mathrm{d}^{a}
$$

where repeated color indices $a$ and $b$ are are always summed over. 
The vacuum saturated four-point functions have the general form of a product of a configuration averaged two-point function and a configuration averaged threepoint function. In the case of Eq. (4.5.31), Eq. (4.5.34), and Eq. (4.5.36) the product is multiplication of two terms that are traces in color space

$$
G^{(4)}\left(t_{1}, t_{2}, t_{3}, 0\right)=\left\langle G^{(2)}\left(t_{1}, 0 ; \mathcal{U}\right)^{a a}\right\rangle_{\{U\}}\left\langle G^{(3)}\left(t_{2}, t_{3}, 0 ; \mathcal{U}\right)^{b b}\right\rangle_{\{U\}}
$$

while for Eq. (4.5.32), Eq. (4.5.33), and Eq. (4.5.35) the product is matrix multiplication in color space and the vacuum saturated four-point function is found by taking the trace in color space of this matrix product

$$
G^{(4)}\left(t_{1}, t_{2}, t_{3}, 0\right)=\left\langle G^{(2)}\left(t_{1}, 0 ; \mathcal{U}\right)^{a b}\right\rangle_{\{U\}}\left\langle G^{(3)}\left(t_{2}, t_{3}, 0 ; \mathcal{U}\right)^{b a}\right\rangle_{\{\mathcal{U}\}}
$$

The notation $G^{(n)}\left(t_{1}, 0 ; \mathcal{U}\right)$ denotes the n-point function calculated in the background gauge field of configuration $\mathcal{U}$.

The additional lattice two-point correlation functions that must be calculated to implement vacuum saturation are:

$$
\begin{aligned}
G_{\pi \Gamma}^{(2)}\left(t_{\pi}, 0\right)_{\mu}^{a b} & =\sum_{\vec{x}_{\pi}}\left\langle 0\left|\chi_{\pi}\left(x_{\pi}\right) \overline{\mathrm{u}}^{a} \Gamma_{\mu}^{A} \mathrm{~d}^{b}(0)\right| 0\right\rangle \\
& =i\left\langle\operatorname{Tr}_{s}\left(\Gamma_{\mu}^{A} \gamma^{5} \sum_{\vec{x}_{\pi}} \mathrm{D}^{\dagger}\left(x_{\pi}, 0 ; \mathcal{U}\right) \mathrm{U}\left(x_{\pi}, 0 ; \mathcal{U}\right)\right)^{b a}\right\rangle \\
G_{K \Gamma}^{(2)}\left(t_{K}, 0\right)_{\mu}^{a b} & =\sum_{\vec{x}_{K}}\left\langle 0\left|\chi_{K}\left(x_{K}\right) \overline{\mathrm{s}}^{a} \Gamma_{\mu}^{A} \mathrm{~d}^{b}(0)\right| 0\right\rangle \\
& =i\left\langle\operatorname{Tr}_{s}\left(\Gamma_{\mu}^{A} \gamma^{5} \sum_{\vec{x}_{K}} \mathrm{D}^{\dagger}\left(x_{K}, 0 ; \mathcal{U}\right) \mathrm{S}\left(x_{K}, 0 ; \mathcal{U}\right)\right)^{b a}\right\rangle
\end{aligned}
$$


and

$$
\begin{aligned}
G_{\Gamma D}^{(2)}\left(0, t_{D}\right)_{\mu}^{a b} & =\sum_{\bar{x}_{D}}\left\langle 0\left|\overline{\mathrm{u}}^{a} \Gamma^{A} \mathrm{c}^{b}(0) \chi_{n}^{\dagger}\left(x_{D}\right)\right| 0\right\rangle \\
& =i\left\langle\operatorname{Tr}_{s}\left(\Gamma_{\mu}^{A} \gamma^{5} \sum_{\bar{x}_{D}} \mathrm{C}^{\dagger}\left(x_{D}, 0 ; \mathcal{U}\right) \mathrm{U}\left(x_{D}, 0 ; \mathcal{U}\right)\right)^{b a}\right\rangle .
\end{aligned}
$$

From these correlation functions we wish to extract a matrix element of the operator $\Gamma_{\mu}^{A}$ sandwiched between the vacuum state $\left(J^{P}=0^{+}\right)$and a single particle $\left(0^{-}\right)$state. To enforce parity conservation only the parity changing part of $\Gamma_{\mu}^{A}=(V-A)^{\mu}$ should be computed. Therefore, the operator $\Gamma_{\mu}^{A}$ is restricted to $\Gamma_{\mu}^{A}=-A^{\mu}$.

The lattice three-point functions that must be calculated are:

$$
\begin{array}{r}
G_{K \Gamma D}^{(3)}\left(t_{K}, 0, t_{D}\right)_{\mu}^{b a}=\sum_{\vec{x}_{K} \vec{x}_{D}}\left\langle 0\left|\chi_{K}\left(x_{K}\right) \overline{\mathrm{s}}^{b} \Gamma^{B} \mathrm{c}^{a}(0) \chi_{D}^{\dagger}\left(x_{D}\right)\right| 0\right\rangle \\
=\left\langle\operatorname{Tr}_{s}\left(\Gamma_{\mu}^{B} \gamma^{5} \sum_{\vec{x}_{D}} \mathrm{C}^{\dagger}\left(x_{D}, 0 ; \mathcal{U}\right) \mathrm{U}_{\mathbf{s}}\left(x_{D}, 0 ; t_{K}, \mathcal{U}\right)\right)^{a b}\right\rangle \\
G_{\pi \Gamma D}^{(3)}\left(t_{\pi}, 0, t_{D}\right)_{\mu}^{b a}=\sum_{\vec{x}_{\pi} \vec{x}_{D}}\left\langle 0\left|\chi_{\pi}\left(x_{\pi}\right) \overline{\mathrm{u}}^{b} \Gamma^{B} \mathrm{c}^{a}(0) \chi_{D}^{\dagger}\left(x_{D}\right)\right| 0\right\rangle \\
=\left\langle\operatorname{Tr}_{s}\left(\Gamma_{\mu}^{B} \gamma^{5} \sum_{\vec{x}_{D}} \mathrm{C}^{\dagger}\left(x_{D}, 0 ; \mathcal{U}\right) \mathrm{D}_{\mathbf{u}}\left(x_{D}, 0 ; t_{\pi}, \mathcal{U}\right)\right)^{a b}\right\rangle
\end{array}
$$

and

$$
\begin{array}{r}
G_{K \pi \Gamma}^{(3)}\left(t_{K}, t_{\pi}, 0\right)_{\mu}^{b a}=\sum_{\vec{x}_{K} \vec{x}_{\pi}}\left\langle 0\left|\chi_{K}\left(x_{K}\right) \chi_{\pi}\left(x_{\pi}\right) \overline{\mathrm{s}}^{b} \Gamma^{B} \mathrm{~d}^{a}(0)\right| 0\right\rangle \\
=\left\langle\operatorname{Tr}_{s}\left(\Gamma_{\mu}^{B} \gamma^{5} \sum_{\vec{x}_{\pi}} \mathrm{D}^{\dagger}\left(x_{\pi}, 0 ; \mathcal{U}\right) \mathrm{U}_{\mathbf{s}}\left(x_{\pi}, 0 ; t_{K}, \mathcal{U}\right)\right)^{a b}\right\rangle .
\end{array}
$$

Here $\Gamma_{\mu}^{B}=(V-A)^{\mu}$ and once again enforcing parity conservation will restrict this operator. The desired matrix element is for $0^{-} \rightarrow 0^{-}$for the first two equations 
or $0^{+} \rightarrow 0^{-} 0^{-}$for the last equation. In either case the parity conserving piece of the operator is required. Thus for these three-point functions $\Gamma_{\mu}^{B}=V^{\mu}$.

The vacuum saturation functions do not require any new combinations of "condensed" quark propagator files beyond those required to calculate the four-point functions. Unlike the four-point functions which are scalars these two-point and three-point functions are $3 \times 3$ matrices in color space and they transform like a four-vector in spin space. In the section on discrete symmetries to follow the number of non-zero elements that must be calculated will be found to be reduced.

\subsection{6. $\quad D^{0} \rightarrow \kappa$ (ds scalar)}

According to Eq. (4.4.23) the Euclidean $D^{0} \rightarrow K \pi$ amplitudes will have a contribution from an intermediate scalar state $(\kappa)$ with valence quark content $\overline{\mathrm{d}} \mathrm{s}$.

We would like to calculate the amplitude

$$
\langle\kappa(\overrightarrow{0})|\mathcal{O}| D(\overrightarrow{0})\rangle
$$

on the lattice where $\mathcal{O}$ is the weak operator. The lattice diagrams for the additional three-point functions required are shown in 4.5.2.

Taking $\chi_{\kappa}=\overline{\mathrm{d} s}$ as the interpolating field, the needed correlation functions are:

$$
\begin{aligned}
& \text { fig8scalar }_{1}\left(t_{s c}, 0, t_{D}\right)= \\
& \qquad i\left\langle\operatorname { T r } _ { s c } \left(\Gamma^{\mu} \gamma^{5} \sum_{\vec{x}_{D}} C^{\dagger}\left(x_{D}, 0 ; \mathcal{U}\right) \mathrm{U}\left(x_{D}, 0 ; \mathcal{U}\right)\right.\right. \\
&\left.\left.\Gamma^{\mu} \gamma^{5} \sum_{\vec{x}_{s c}} \mathrm{D}^{\dagger}\left(x_{s c}, 0 ; \mathcal{U}\right) \gamma^{5} \mathrm{~S}\left(x_{s c}, 0 ; \mathcal{U}\right)\right)\right\rangle
\end{aligned}
$$




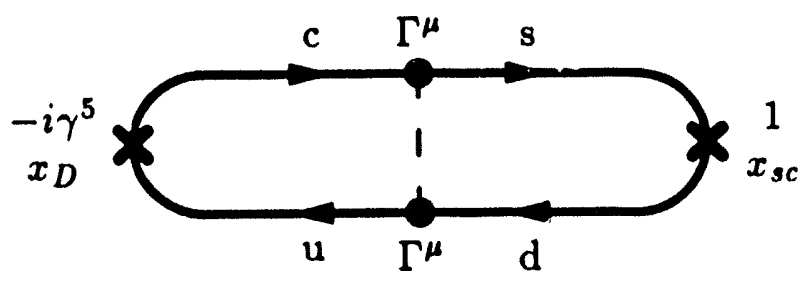

\section{fig8scalar $1_{1}$}

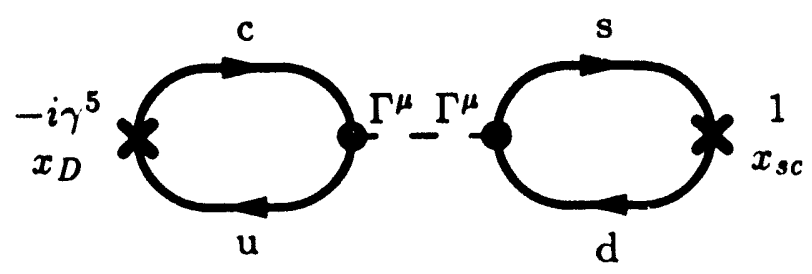

fig8scalar 2

Figure 4.5.2: Lattice diagrams corresponding to the three-point functions needed for $D^{0} \rightarrow \kappa$.

and

$$
\begin{aligned}
& \text { fig8scalar }_{2}\left(t_{s c}, 0, t_{D}\right)= \\
&-i\left\langle\operatorname{Tr}_{s c}\left(\Gamma^{\mu} \gamma^{5} \sum_{\vec{x}_{D}} \mathrm{C}^{\dagger}\left(x_{D}, 0 ; \mathcal{U}\right) \mathrm{U}\left(x_{D}, 0 ; \mathcal{U}\right)\right)\right. \\
&\left.\operatorname{Tr}_{s c}\left(\Gamma^{\mu} \gamma^{5} \sum_{\vec{x}_{s c}} \mathcal{D} \dagger\left(x_{s c}, 0 ; \mathcal{U}\right) \gamma^{5} \mathrm{~S}\left(x_{s c}, 0 ; \mathcal{U}\right)\right)\right\rangle
\end{aligned}
$$

for the $\mathcal{O}_{1}$ and $\mathcal{C}_{2}$ operators respectively.

The fig8scalar functions require an additional condensing of the scalar type. The combination of the $d$ and s quark propagators

$$
\sum_{\vec{x}_{s c}} \mathrm{D}^{\dagger}\left(x_{s c}, 0 ; \mathcal{U}\right) \gamma^{5} \mathrm{~S}\left(x_{s c}, 0 ; \mathcal{U}\right)
$$


is precomputed and stored for later use in the condensing step. Note that the $\gamma^{5}$ matrix between the two propagators corresponds to the insertion of a unit matrix at $x_{s c}$ in 4.5 .2 .

So far the form

$$
\Gamma^{\mu} \otimes \Gamma^{\mu}=(V-A)^{\mu} \otimes(V-A)^{\mu}
$$

has been assumed for the weak operators. The initial state $D$ has quantum numbers $0^{-}$while the final state $\kappa$ has the assignment $0^{+}$. Thus we can restrict the operator to its parity changing part. Therefore, take the operator structure to be

$$
\Gamma^{\mu} \otimes \Gamma^{\mu}=-\left(V^{\mu} \otimes A^{\mu}+A^{\mu} \otimes V^{\mu}\right)
$$

for the fig8scalar functions.

\subsection{Dimensionful Four-point Functions}

The four-point functions of the preceding section are calculated with field operators scaled to make them dimensionless and to absorb some overall factors of the hopping constants. These factors must be restored in order that the $\mathrm{n}$-point functions will have the proper engineering dimensions.

Section 4.2 demonstrates how to restore the proper dimensional units to propagators. The same procedure is now applied to the four-point functions calculated on the lattice.

The four-point functions are of the form

$$
\begin{aligned}
& G^{(4)}\left(t_{K}, t_{\pi}, 0, t_{D}\right)= \\
& \quad \sum_{\vec{x}_{K} \vec{x}_{\pi} \vec{x}_{D}}\left\langle 0\left|T\left(\chi_{K}\left(x_{K}\right) \chi_{\pi}\left(x_{\pi}\right) \mathcal{O}(0) \chi_{D}^{\dagger}\left(x_{D}\right)\right)\right| 0\right\rangle
\end{aligned}
$$


The relation Eq. (4.2.14) restores the dimensional units to the quark fields and Eq. (4.2.15) is the corresponding relation for the interpolating fields. Remembering that $\mathcal{O}$ is a four-Fermion operator and that there is a factor of $a^{3}$ from the sums over $\vec{x}$ one finds that

$$
G^{(4)}\left(t_{K}, t_{\pi}, 0, t_{D}\right) \rightarrow 2^{5} \kappa_{u, d}^{3} \kappa_{s} \kappa_{c} G^{(4)}\left(t_{K}, t_{\pi}, 0, t_{D}\right)
$$

where $\kappa_{u, d}$ is the hopping constant for the degenerate $\mathrm{u}$ and $\mathrm{d}$ quarks and $\kappa_{s}$ and $\kappa_{c}$ are respectively the strange quark and the charm quark hopping constants. Notice that the powers of the lattice spacing $a$ have canceled for the four-point correlation function. This rescaling also applies to the vacuum saturated four-point functions $G^{(4)}{ }^{V S}\left(t_{K}, t_{\pi}, 0, t_{D}\right)$ in equations Eq. (4.5.31) through Eq. (4.5.36).

A lattice matrix element is extracted from a four-point function by making use of the results of section 4.4. According to Eq. (4.4.22) the large time behavior of the "time-independent" (TI) ratio, formed by dividing the four-point function by the propagator functions corresponding to the legs of the four-point function and then multiplying the normalization constants $Z$ obtained from the propagator, contains a term equal to the desired matrix element. The dimensional factor for a lattice matrix element is the same as the dimensional factor for the corresponding TI ratio. From Eq. (4.6.2) along with the dimensional factor for propagators Eq. (4.2.16) and the dimensional factor for the normalization constant $Z$ Eq. (4.2.17) one finds by substitution into Eq. ( $\therefore$. 2 ?) that the proper dimensiunal factor is restored to the "time-independent" ratio $R$ for four-point functions by the replicement

$$
R^{(4)} \rightarrow \frac{\sqrt{16 \kappa_{u, d}^{2} \kappa_{g} \kappa_{c}}}{a^{3}} R^{(4)}
$$


Here we see that the lattice $D \rightarrow K \pi$ matrix elements depend on the cube of the inverse lattice spacing. It is commonly believed that the lattice spacing is determined to a precision of roughly twenty percent from string tension measurements. The dependence of matrix elements upon powers of $a^{-1}$ then leads to a large systematic uncertainty in the determination of lattice matrix elements.

\subsection{Discrete Symmetries}

In this section the discrete symmetries of the gauge configurations and quark propagators: parity Eq. (2.6.10), time reflection Eq. (2.6.12), charge conjugation Eq. (2.6.7) and Hermiticity Eq. (2.6.4) are applied to lattice correlation functions. The generation of new gauge corfigurations and the corresponding multitude of quark propagators and exponentiated propagators for the various hopping constants that must also be generated is costly in both machine and human time. It is then important to use as fully as possible the information in a given configuration. Discrete symmetries are useful for this purpose.

Suppose a single configuration $\mathcal{U}$ is generated by Monte Carlo methods. Since charge conjugation, parity, and time reflection are symmetries of the gauge action Eq. (2.2.6), the set of all possible configurations $\{\mathcal{U}\}_{\text {all }}$ will also contain $\mathcal{U}^{S}$ where $S=C, P, T, C P, \ldots$ the symmetry transformed partners of the original configuration. These additional configurations can be added to the set of configurations $\{\mathcal{U}\}$ generated by Monte Carlo methods. Instead of averaging a physical obseivable $\theta(\mathcal{U})$ over just the original sample of gauge configurations $\langle\theta(\mathcal{U})\rangle_{\{\mathcal{U}\}}$ the average is now performed over the original set of configurations and the set of symmetry transformed contigurations $\langle\theta(\mathcal{U})\rangle_{\{\mathcal{U}\} \cup\left\{\mathcal{U}^{s}\right\}}$. Increasing the number of configurations should then lead to a reduction in the statistical error due to 
the finite configuration sample size. The original configuration and the symmetry transformed configurations are correlated so the reduction in statistical error is less than the reduction that comes from increasing the number of uncorrelated configurations.

The method described above would still be too expensive since to compute the observables of interest in weak matrix element calculations would require the calculation of $\mathrm{Q}\left(x, y ; \mathcal{U}^{S}\right)$ the quark propagators in the fixed background of the symmetry transformed configurations. Instead of explicitly transforming the gauge configurations to generate $\mathcal{U}^{S}$ and then calculating the quark propagators in the background field of the transformed configurations, consider using the discrete symmetries to transform the quark propagators. We list Eq. (2.6.7), Eq. (2.6.10) and Eq. (2.6.12) the symmetry identities for the quark propagators:

$$
\begin{aligned}
& \mathrm{Q}\left(x, y, \mathcal{U}^{C}\right)=C \mathrm{Q}^{T}(y, x ; \mathcal{U}) C^{-1} \\
& \mathrm{Q}\left(x, y, \mathcal{U}^{P}\right)=\gamma^{4} \mathrm{Q}\left(x^{P}, y^{P} ; \mathcal{U}\right) \gamma^{4} \\
& \mathrm{Q}\left(x, y, \mathcal{U}^{T}\right)=T \mathrm{Q}\left(x^{T}, y^{T} ; \mathcal{U}\right) T^{-1}
\end{aligned}
$$

Thus, using these relations the configuration average of an observable $\theta(\mathcal{U})$ over the original configurations and over the symmetry transformed configurations is equivalent to configuration averaging over just the original sample of gauge configurations the average of the observable and its symmetry transformed counterpart: $\left\langle\left(\theta(\mathcal{U})+\theta^{S}(\mathcal{U})\right) / 2\right\rangle_{\{\mathcal{U}\}}$. 


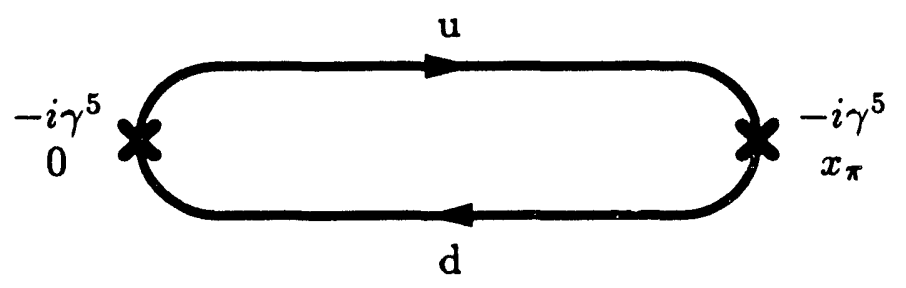

Figure 4.7.1: Lattice diagram corresponding to the $\pi^{+}$propagator $G_{\pi^{+}}^{(2)}(t, 0 ; \vec{p})$.

\subsubsection{Application to Meson Propagators}

The discrete symmetries are applied to the propagators. As an example, consider the $\pi^{+}$propagator Eq. (4.2.3)

$$
G_{\pi^{+}}^{(2)}(t, 0 ; \vec{p})=\left\langle G_{\pi^{+}}^{(2)}(t, 0 ; \vec{p}, \mathcal{U})\right\rangle_{\{\mathcal{U}\}}
$$

shown in Fig. 4.7.1 where brackets indicate an average over the Monte Carlo sample of gauge configurations $\{\mathcal{U}\}$ and the quantity

$$
\begin{aligned}
& G_{\pi^{+}}^{(2)}(t, 0 ; \vec{p}, \mathcal{U})= \\
& \quad \sum_{\vec{x}} e^{\frac{2 \pi i}{L} \vec{p} \cdot \vec{x}} \operatorname{Tr}_{s c}\left(\gamma^{5} \mathrm{D}(0, x ; \mathcal{U}) \gamma^{5} \mathrm{U}(x, 0 ; \mathcal{U})\right)
\end{aligned}
$$

is the $\pi^{+}$propagator in the fixed background gauge configuration $\mathcal{U}$.

Now calculate $G_{\pi^{+}}^{(2)}\left(t, 0 ; \vec{p}, \mathcal{U}^{P}\right)$ the function that results from using the parity reversed gauge configuration $\mathcal{U}^{P}$. Substituting $\mathcal{U}^{P}$ into Eq. (4.7.3) and using Eq. (4.7.1b) along with the relation $\gamma^{4} \gamma^{5} \gamma^{4}=-\gamma^{5}$ yields

$$
\begin{aligned}
& G_{\pi^{+}}^{(2)}\left(t, 0 ; \vec{p}, \mathcal{U}^{P}\right)= \\
& \quad \sum_{\vec{x}} e^{\frac{2 \pi i}{L} \vec{p} \cdot \vec{x}} \operatorname{Tr}_{s c}\left(\gamma^{5} \mathrm{D}\left(0, x^{P} ; \mathcal{U}\right) \gamma^{5} \mathrm{U}\left(x^{P}, 0 ; \mathcal{U}\right)\right)
\end{aligned}
$$


Writing $x=(t, \vec{x})$ then $x^{P}=(t,-\vec{x})$ represents the parity transformed coordinates. Note that $\vec{p} \cdot \vec{x}=\vec{p}^{P} \cdot \vec{x}^{P}$ and that the sum over all $\vec{x}$ in Eq. (4.7.4) can be replaced by a sum over $\vec{x}^{P}$. Then,

$$
G_{\pi^{+}}^{(2)}\left(t, 0 ; \vec{p}, \mathcal{U}^{P}\right)=G_{\pi^{+}}^{(2)}\left(t, 0 ;-\vec{p}, \mathcal{U}^{\prime}\right.
$$

and the effect of averaging over the parity reversed configurations can be included by making the replacement

$$
G_{\pi^{+}}^{(2)}(t, 0 ; \vec{p}, \mathcal{U}) \rightarrow \frac{1}{2}\left[G_{\pi^{+}}^{(2)}(t, 0 ; \vec{p}, \mathcal{U})+G_{\pi^{+}}^{(2)}(t, 0 ;-\vec{p}, \mathcal{U})\right]
$$

in Eq. (4.7.2).

Now return to Eq. (4.7.3) and replace the configuration with the charge conjugate configuration $\mathcal{U}^{C}$. The identity for quark propagators Eq. (4.7.1a) is then used to rewrite the propagator again in terms of the original configuration $\mathcal{U}$

$$
\begin{aligned}
& G_{\pi^{+}}^{(2)}\left(t, 0 ; \vec{p}, \mathcal{U}^{C}\right)= \\
& \quad \sum_{\vec{x}} e^{\frac{2 \pi i}{L} \vec{p} \cdot \vec{x}} \operatorname{Tr}_{s c}\left(C^{-1} \gamma^{5} C \mathrm{D}^{T}(x, 0 ; \mathcal{U}) C^{-1} \gamma^{5} C \mathrm{U}^{T}(0, x ; \mathcal{U})\right)
\end{aligned}
$$

where the notation $Q^{T}$ represents the transpose of matrix $Q$. Recall that $C=\gamma^{4} \gamma^{2}$ so that $C^{-1} \gamma^{5} C=\gamma^{5}$. Then using the Hermiticity property Eq. (2.6.4) for quark propagators and that $\gamma^{5}=\gamma^{5^{*}}$, the propagator in the fixed background of the charge conjugate gauge field becomes

$$
\begin{aligned}
& G_{\pi^{+}}^{(2)}\left(t, 0 ; \vec{p}, \mathcal{U}^{C}\right)= \\
& \quad \sum_{\vec{x}} e^{\frac{2 \pi i}{L} \vec{p} \cdot \vec{x}} \operatorname{Tr}_{s c}\left(\gamma^{5} \mathrm{D}(0, x ; \mathcal{U}) \gamma^{5} U(x, 0 ; \mathcal{U})\right)^{*}
\end{aligned}
$$


where the star operation is the complex conjugation of the trace. The previous equation then simplifies to

$$
G_{\pi^{+}}^{(2)}\left(t, 0 ; \vec{p}, \mathcal{U}^{C}\right)=G_{\pi^{+}}^{(2)}(t, 0 ;-\vec{p}, \mathcal{U})^{*}
$$

The propagator can effectively be averaged over the set of configurations $\{\mathcal{U}\} \cup$ $\left\{\mathcal{U}^{P}\right\} \cup\left\{\mathcal{U}^{C}\right\} \cup\left\{\mathcal{U}^{C P}\right\}$ by combining result Eq. (4.7.6) for parity with the result above for charge conjugation. The effect of these combined symmetries can be included by the replacement

$$
\begin{aligned}
& G_{\pi^{+}}^{(2)}(t, 0 ; \vec{p}, \mathcal{U}) \rightarrow \\
& \quad \frac{1}{2} \operatorname{Re}\left[G_{\pi^{+}}^{(2)}(t, 0 ; \vec{p}, \mathcal{U})+G_{\pi^{+}}^{(2)}(t, 0 ;-\vec{p}, \mathcal{U})\right]
\end{aligned}
$$

in the configuration average Eq. (4.7.2). Nutice that the propagator is real (by making the configurations $C P$ symmetric).

The effective set of configurations can be further extended by including the time reflected configurations $\left\{\mathcal{U}^{T}\right\}$ in the configuration average. Replacing $\mathcal{U}$ by $\mathcal{U}^{T}$ in function Eq. (4.7.3) and using identity Eq. (4.7.1c) yields

$$
\begin{aligned}
& G_{\pi^{+}}^{(2)}\left(t, 0 ; \vec{p}, \mathcal{U}^{T}\right)= \\
& \quad \sum_{\vec{x}} e^{\frac{2 \pi i}{L} \vec{p} \cdot \vec{x}} \operatorname{Tr}_{s c}\left(T^{-1} \gamma^{5} T \mathrm{D}\left(0, x^{T} ; \mathcal{U}\right) T^{-1} \gamma^{5} T \mathrm{U}\left(x^{T}, 0 ; \mathcal{U}\right)\right)
\end{aligned}
$$

where the symbol $T$ in the notation $x^{T}$ is defined by $x^{T}=(-t, \vec{x})$ with $x=(t, \vec{x})$. The matrix $T$ is defined by $T=\gamma^{4} \gamma^{5}$. Then $T^{-1} \gamma^{5} T=-\gamma^{5}$ so that

$$
G_{\pi^{+}}^{(2)}\left(t, 0 ; \vec{p}, \mathcal{U}^{T}\right)=G_{\pi^{+}}^{(2)}(-t, 0 ; \vec{p}, \mathcal{U}) .
$$

Including the time reflected configurations $\mathcal{U}^{T}$ is then equivalent to configuration averaging the time symmetrized quantity

$$
\frac{1}{2}\left[G_{\pi^{+}}^{(2)}(t, 0 ; \vec{p}, \mathcal{U})+G_{\pi^{+}}^{(2)}(-t, 0 ; \vec{p}, \mathcal{U})\right]
$$


In practice, the results of Eq. (4.7.10) and Eq. (4.7.13) can be combined. Propagators for fixed $t$ are calculated according to Eq. (4.7.10) the sign of $t$ is then reversed and the calculation is repeated. The results for positive and negative times are then averaged. In the lattices used in this calculation the origin of coordinates is taken at the center of the lattice allowing the time averaging to be done.

The results Eq. (4.7.10) and Eq. (4.7.13) were demonstrated for the pseudoscalar case. These results are also true for scalar propagators.

\subsubsection{Application to Fig8 Functions}

In the previous discussion of the fig8 correlation functions (see Sect. 4.5) the effective weak operators were written in the general form

$$
\begin{aligned}
& \mathcal{O}_{1}=\overline{\mathrm{s}} \Gamma^{A} \mathrm{c} \overline{\mathrm{u}} \Gamma^{B} \mathrm{~d} \\
& \mathcal{O}_{2}=\overline{\mathrm{s}} \Gamma^{B} \mathrm{~d} \overline{\mathrm{u}} \Gamma^{A} \mathrm{c}
\end{aligned}
$$

where according to the derivation of the effective weak operator on the lattice, the big gamma matrices are $\Gamma^{A}=\Gamma^{B}=\gamma^{\mu}\left(1-\gamma^{5}\right)=(V-A)^{\mu}$, the last form making explicit the vector minus axial vector form of the current.

To simplify the discussion use the notation $\Gamma^{A} \otimes \Gamma^{B}$ to represent the Dirac structure of the operators Eq. (4.7.14). Hence, the effective weak operators are of the form $(V-A)^{\mu} \otimes(V-A)^{\mu}$. For decays of the type $0^{-} \rightarrow 0^{-} 0^{-}$, such as the $D \rightarrow K \pi$ modes, enforcing parity conservation will restrict the operators to the parity changing part so that $\Gamma^{A} \otimes \Gamma^{B}=-V^{\mu} \otimes A^{\mu}-A^{\mu} \otimes V^{\mu}$.

The structure of the operator used in a correlation function will be indicated by a subscript: $G^{(4)}\left(t_{K}, t_{\pi}, 0, t_{D}\right) \Gamma \otimes \Gamma$ when it is important to emphasize the form of the operator. 
As an example of a four-point function consider fig8 $\pi_{1}$ in Eq. (4.5.13):

$$
\text { fig8 } 8 \pi_{1}\left(t_{K}, t_{\pi}, 0, t_{D}\right)=i\left\langle\operatorname{fig} 8 \pi_{1}\left(t_{K}, t_{\pi}, 0, t_{D} ; \mathcal{U}\right)_{\Gamma^{A} \otimes \Gamma^{B}}\right\rangle_{\{U\}}
$$

where the correlation function in the fixed background gauge configuration $\mathcal{U}$ is

$$
\begin{aligned}
& \text { fig8 } 8 \pi_{1}\left(t_{K}, t_{\pi}, 0, t_{D} ; \mathcal{U}\right)_{\Gamma^{A} \otimes \Gamma^{B}}= \\
& \qquad \begin{array}{l}
\sum_{\vec{x}_{D} \vec{x}_{K} \vec{x}_{\pi}} \operatorname{Tr}_{s c}\left(\Gamma^{B} \mathrm{D}\left(0, x_{\pi} ; \mathcal{U}\right) \gamma^{5} \mathrm{U}\left(x_{\pi}, 0 ; \mathcal{U}\right)\right) \\
\operatorname{Tr}_{s c}\left(\Gamma^{A} \mathrm{C}\left(0, x_{D} ; \mathcal{U}\right) \gamma^{5} \mathrm{U}\left(x_{D}, x_{K} ; \mathcal{U}\right) \gamma^{5} \mathrm{~S}\left(x_{K}, 0 ; \mathcal{U}\right)\right)
\end{array}
\end{aligned}
$$

and the operator structure is $\Gamma^{A} \otimes \Gamma^{B}=-V^{\mu} \otimes A^{\mu}-A^{\mu} \otimes V^{\mu}$.

Consider substituting the parity reversed configuration $\mathcal{U}^{P}$ into Eq. (4.7.16). Making use of the identity Eq. (4.7.1b) the function becomes

$$
\begin{aligned}
\text { fig8 } \pi_{1}\left(t_{K}, t_{\pi}, 0, t_{D} ; \mathcal{U}^{P}\right)= \\
\quad \sum_{\vec{x}_{D}^{P} \vec{x}_{K}^{P} \vec{x}_{\pi}^{P}} \operatorname{Tr}_{s c}\left(-\gamma^{4} \Gamma^{B} \gamma^{4} \mathrm{D}\left(0, x^{P} ; \mathcal{U}\right) \gamma^{5} \mathrm{U}\left(x^{P}, 0 ; \mathcal{U}\right)\right) \\
\operatorname{Tr}_{s c}\left(\gamma^{4} \Gamma^{A} \gamma^{4} \mathrm{C}\left(0, x^{P}{ }_{D} ; \mathcal{U}\right) \gamma^{5} \mathrm{U}\left(x^{P}{ }_{D}, x^{P}{ }_{K} ; \mathcal{U}\right) \gamma^{5} \mathrm{~S}\left(x^{P}{ }_{K}, 0 ; \mathcal{U}\right)\right)
\end{aligned}
$$

where the relation $\gamma^{4} \gamma^{5} \gamma^{4}=-\gamma^{5}$ has also been used. Now

$$
\gamma^{4} \Gamma^{A} \gamma^{4} \otimes \gamma^{4} \Gamma^{B} \gamma^{4}=-\Gamma^{A} \otimes \Gamma^{B}
$$

so that

$$
\text { fig8 } 8 \pi_{1}\left(t_{K}, t_{\pi}, 0, t_{D} ; \mathcal{U}^{P}\right)=\text { fig } 8 \pi_{1}\left(t_{K}, t_{\pi}, 0, t_{D} ; \iota !\right.
$$

Therefore averaging over the parity reversed configurations has no effect for the at rest four-point functions. Next consider including the charge conjugate configurations $\left\{\mathcal{U}^{C}\right\}$ in the configuration average. Begin with Eq. (4.7.16) and substitute 
the configuration $\mathcal{U}^{C}$ for $\mathcal{U}$. Use Eq. (4.7.1a) and Hermiticity of the quark propagators to get

$$
\begin{aligned}
& \text { fig8 } \pi_{1}\left(t_{K}, t_{\pi}, 0, t_{D} ; \mathcal{U}^{C}\right)= \\
& \sum_{\vec{x}_{D}^{P} \vec{x}_{K}^{P} \vec{x}_{\pi}^{P}} \operatorname{Tr}_{s c}\left(\gamma^{5} C^{-1} \Gamma^{B} C \gamma^{5} D^{*}\left(0, x_{\pi} ; \mathcal{U}\right) \gamma^{5} U^{*}\left(x_{\pi}, 0 ; \mathcal{U}\right)\right) \\
& \operatorname{Tr}_{s c}\left(\gamma^{5} C^{-1} \Gamma^{A} C \gamma^{5} C^{*}\left(0, x_{D} ; \mathcal{U}\right) \gamma^{5} U^{*}\left(x_{D}, x_{K} ; \mathcal{U}\right) \gamma^{5} S^{*}\left(x_{K}, 0 ; \mathcal{U}\right)\right)
\end{aligned}
$$

where $C=\gamma^{4} \gamma^{2}$ so that

$$
\gamma^{5} C^{-1} \Gamma^{A} C \gamma^{5} \otimes \gamma^{5} C^{-1} \Gamma^{B} C \gamma^{5}=\left(\Gamma^{A} \otimes \Gamma^{B}\right)^{*}
$$

Therefore,

$$
\text { fig } 8 \pi_{1}\left(t_{K}, t_{\pi}, 0, t_{D} ; \mathcal{U}^{C}\right)=\text { fig } 8 \pi_{1}\left(t_{K}, t_{\pi}, 0, t_{D} ; \mathcal{U}\right)^{*}
$$

and the effect of including the charge conjugate configuration in the configuration average is equivalent to taking the real part of the four-point function

$$
\text { fig } 8 \pi_{1}\left(t_{K}, t_{\pi}, 0, t_{D} ; \mathcal{U}\right) \rightarrow \operatorname{Refig} \delta \pi_{1}\left(t_{K}, t_{\pi}, 0, t_{D} ; \mathcal{U}\right)
$$

Finally consider including the time reflected configurations $\left\{\mathcal{U}^{T}\right\}$ in the configuration average. From Eq. (4.7.16) and Eq. (4.7.1c) and recalling that $T=\gamma^{4} \gamma^{5}$ gives

$$
\begin{aligned}
& \text { fig8 } 8 \pi_{1}\left(t_{K}, t_{\pi}, 0, t_{D} ; \mathcal{U}^{T}\right)= \\
& \sum_{\vec{x}_{D} \vec{x}_{K} \vec{x}_{\pi}} \operatorname{Tr}_{s c}\left(-T^{-1} \Gamma^{B} \operatorname{TD}\left(0, x^{T} ; \mathcal{U}\right) \gamma^{5} \mathrm{U}\left(x^{T}, 0 ; \mathcal{U}\right)\right) \\
& \operatorname{Tr}_{s c}\left(T^{-1} \Gamma^{A} T \mathrm{C}\left(0, x^{T}{ }_{D} ; \mathcal{U}\right) \gamma^{5} \mathrm{U}\left(x^{T}{ }_{D}, x^{T}{ }_{K} ; \mathcal{U}\right) \gamma^{5} \mathrm{~S}\left(x^{T}{ }_{K}, 0 ; \mathcal{U}\right)\right)
\end{aligned}
$$

where $T^{-1} \gamma^{5} T=-\gamma^{5}$ has also been used. It follows that

$$
T^{-1} \Gamma^{A} T \otimes T^{-1} \Gamma^{B} T=-\Gamma^{A} \otimes \Gamma^{B}
$$


and

$$
\text { fig } 8 \pi_{1}\left(t_{K}, t_{\pi}, 0, t_{D} ; \mathcal{U}^{T}\right)=\operatorname{fig} 8 \pi_{1}\left(-t_{K},-t_{\pi}, 0,-t_{D} ; \mathcal{U}\right)
$$

Therefore, the four-point functions should be time symmetrized in configuration averages.

The final form for fig $8 \pi_{1}$ is then

$$
\begin{aligned}
& \text { fig8 } 8 \pi_{1}\left(t_{K}, t_{\pi}, 0, t_{D}\right)= \\
& \qquad \frac{1}{2} \operatorname{Re}\left[\text { fig8 } 8 \pi_{1}\left(t_{K}, t_{\pi}, 0, t_{D} ; \mathcal{U}\right)+\right. \\
& \quad{\left.\left.\operatorname{fig} 8 \pi_{1}\left(-t_{K},-t_{\pi}, 0,-t_{D} ; \mathcal{U}\right)\right]\right\rangle_{\{U\}}} .
\end{aligned}
$$

The function fig $8 \pi_{1}\left(t_{K}, t_{\pi}, 0, t_{D} ; \mathcal{U}\right)$ defined in Eq. (4.7.16) and is computed with the operator $\Gamma^{A} \otimes \Gamma^{B}=-V^{\mu} \otimes A^{\mu}-A^{\mu} \otimes V^{\mu}$.

These results derived for the correlation function fig8 $8 \pi_{1}$ also hold for the other fig8 functions.

\subsubsection{Application to VS Functions}

Consider the two-point functions that are computed in the vacuum saturation approximation. As an example, take

$$
G_{\pi \Gamma}^{(2)}\left(t_{\pi}, 0\right)_{\mu}^{a b}=i\left\langle G_{\pi \Gamma}^{(2)}\left(t_{\pi}, 0\right)_{\mu}^{a b}\right\rangle_{\{U\}}
$$

of Ey. (4.5.42) where

$$
G_{\pi \Gamma}^{(2)}\left(t_{\pi}, 0 ; \mathcal{U}\right)_{\mu}^{a b}=\operatorname{Tr}_{s}\left(-A^{\mu} \sum_{\vec{x}_{\pi}} \mathrm{D}\left(0, x_{\pi} ; \mathcal{U}\right) \gamma^{5} \mathrm{U}\left(x_{\pi}, 0 ; \mathcal{U}\right)\right)^{b a}
$$


Substituting $\mathcal{U}^{P}$ into Eq. (4.7.29) and using Eq. (4.7.1b) results in

$$
G_{\pi \Gamma}^{(2)}\left(t_{\pi}, 0 ; \mathcal{U}^{P}\right)_{\mu}^{a b}=\operatorname{Tr}_{s}\left(\Gamma^{\mu} \sum_{\vec{x}_{\pi}} \mathrm{D}\left(0, x_{\pi} ; \mathcal{U}\right) \gamma^{5} \mathrm{U}\left(x_{\pi}, 0 ; \mathcal{U}\right)\right)^{b a}
$$

where $\Gamma^{\mu}=\left(-A^{4}, \vec{A}\right)$.

Then averaging Eq. (4.7.29) and Eq. (4.7.30) is equivalent to the replacement

$$
G_{\pi \Gamma}^{(2)}\left(t_{\pi}, 0 ; \mathcal{U}\right)_{\mu}^{a b} \rightarrow\left\{\begin{array}{cc}
G_{\pi \Gamma}^{(2)}\left(t_{\pi}, 0 ; \mathcal{U}\right)_{\mu}^{a b} & \mu=4 \\
0 & \mu \neq 4
\end{array}\right.
$$

with $G^{(2)}$ defined as in Eq. (4.7.29). For the "at rest" two-point vacuum insertion functions only the time component is retained.

Substituting $\mathcal{U}^{C}$ for $\mathcal{U}$ in Eq. (4.7.31) and using Eq. (4.7.1a) along with the identity $\gamma^{5} C^{-1} A^{4} C \gamma^{5}=\left(A^{4}\right)^{*}$ then yields

$$
G_{\pi \Gamma}^{(2)}\left(t_{\pi}, 0 ; \mathcal{U}^{C}\right)_{4}^{a b}=G_{\pi \Gamma^{*}}^{(2)}\left(t_{\pi}, 0 ; \mathcal{U}\right)_{4}^{a b^{*}}
$$

The effect of including the configurations $\mathcal{U}^{C}$ is then the same as the replacement

$$
G_{\pi \Gamma}^{(2)}\left(t_{\pi}, 0 ; \mathcal{U}^{C}\right)_{4}^{a b} \rightarrow \operatorname{Re} G_{\pi \Gamma}^{(2)}\left(t_{\pi}, 0 ; \mathcal{U}\right)_{4}^{a b}
$$

in the configuration average.

Next consider the effect of including the configuration $\mathcal{U}^{T}$. Making this replacement in Eq. (4.7.29) and using $T^{-1} A^{4} T=A^{4}$ then gives

$$
G_{\pi \Gamma}^{(2)}\left(t_{\pi}, 0 ; \mathcal{U}^{T}\right)_{4}^{a b}=-G_{\pi \Gamma}^{(2)}\left(-t_{\pi}, 0 ; \mathcal{U}\right)_{4}^{a b}
$$

In summary, the function $G_{\pi \Gamma}^{(2)}\left(t_{\pi}, 0\right)_{\mu}^{a b}$ is computed according to

$$
\begin{aligned}
& G_{\pi \Gamma}^{(2)}\left(t_{\pi}, 0\right)_{4}^{a b}= \\
& \quad i\left\langle\frac{1}{2} \operatorname{Re}\left[G_{\pi \Gamma}^{(2)}\left(t_{x}, 0 ; \mathcal{U}\right)_{4}^{a b}-G_{\pi \Gamma}^{(2)}\left(-t_{\pi}, 0 ; \mathcal{U}\right)_{4}^{a b}\right]\right\rangle_{\{U\}}
\end{aligned}
$$


for $\mu=4$ and $G_{\pi \Gamma}^{(2)}\left(t_{\pi}, 0\right)_{\mu}^{a b}=0$ for $\mu \neq 4$.

Now consider the three-point vacuum saturation function

$$
G_{K \Gamma D}^{(3)}\left(t_{K}, 0, t_{D}\right)_{\mu}^{b a}=\left\langle G_{K \Gamma D}^{(3)}\left(t_{K}, 0, t_{D} ; \mathcal{U}\right)_{\mu}^{b a}\right\rangle_{\{U\}}
$$

where the three-point function in the fixed background gauge field $\mathcal{U}$ is

$$
\begin{aligned}
& G_{K \Gamma D}^{(3)}\left(t_{K}, 0, t_{D} ; \mathcal{U}\right)_{\mu}^{b a}= \\
& \quad \operatorname{Tr}_{s}\left(V^{\mu} \sum_{\vec{x}_{K} \vec{x}_{D}} \mathrm{C}\left(0, x_{D} ; \mathcal{U}\right) \gamma^{5} \mathrm{U}\left(x_{D}, x_{K} ; \mathcal{U}\right) \gamma^{5} \mathrm{~S}\left(x_{K}, 0 ; \mathcal{U}\right)\right)^{a b}
\end{aligned}
$$

Averaging Eq. (4.7.37) over $\mathcal{U}$ and $\mathcal{U}^{P}$ is equivalent to the replacement

$$
G_{K \Gamma D}^{(3)}\left(t_{K}, 0, t_{D} ; \mathcal{U}\right)_{\mu}^{b a} \rightarrow\left\{\begin{array}{cc}
G_{K \Gamma D}^{(3)}\left(t_{K}, 0, t_{D} ; \mathcal{U}\right)_{\mu}^{b a} & \mu=4 \\
0 & \mu \neq 4
\end{array}\right.
$$

in Eq. (4.7.37) as one can easily verify. As a check, note that for the two-point vacuum saturation function results only the $\mu=4$ component survives when considering "at rest" amplitudes. Since the vacuum saturated four-point function is a product of two-point and three-point functions summed over $\mu$, only the $\mu=4$ componerit of the three-point function is important.

Likewi:e, including the set $\left\{\mathcal{U}^{C}\right\}$ in the configuration average can be shown to be the same as taking only the real part of the three-point function Eq. (4.7.38).

Now consider replacing $\mathcal{U}$ by $\mathcal{U}^{T}$ in Eq. (4.7.37). From Eq. (4.7.1c) and the identities $T^{-1} V^{4} T=-V^{4}$ and $T^{-1} \gamma^{5} T=-\gamma^{5}$ it fcilows that

$$
G_{K \Gamma D}^{(3)}\left(t_{K}, 0, t_{D} ; \mathcal{U}^{T}\right)_{4}^{b a}=-G_{K \Gamma D}^{(3)}\left(-t_{K}, 0,-t_{D} ; \mathcal{U}\right)_{4}^{b a} .
$$

Combining these results together the final form of the three-point function is

$$
\begin{aligned}
& G_{K \Gamma D}^{(3)}\left(t_{K}, 0, t_{D}\right)_{4}^{b a} \rightarrow \\
& \left\langle\frac { 1 } { 2 } \operatorname { R e } \left[ G_{K^{\prime} \Gamma D}^{(3)}\left(t_{K}, 0, t_{D} ; \mathcal{U}\right)_{4}^{b a}-\right.\right. \\
& \left.\left.G_{K^{\prime} \Gamma D}^{(3)}\left(-t_{K}, 0,-t_{D} ; \mathcal{U}\right)_{4}^{b a}\right]\right\rangle
\end{aligned}
$$


while for $\mu \neq 4$ the components are zero.

\subsubsection{Application to fig8scalar functions}

As usual, define

$$
\text { fig8scalar }_{2}\left(t_{s c}, 0, t_{D}\right)=-i\left\langle\operatorname{fig} 8 s c a l a r_{2}\left(t_{s c}, 0, t_{D} ; \mathcal{U}\right)\right\rangle_{\{U\}}
$$

with the correlation function calculated in the configuration $\mathcal{U}$ given by

$$
\begin{aligned}
\text { fig8scalar }_{2}\left(t_{s c}, 0, t_{D} ; \mathcal{U}\right)= \\
\qquad \operatorname{Tr}_{s c}\left(\Gamma^{\mu} \sum_{\vec{x}_{D}} \mathrm{C}\left(0, x_{D} ; \mathcal{U}\right) \gamma^{5} \mathrm{U}\left(x_{D}, 0 ; \mathcal{U}\right)\right) \\
\operatorname{Tr}_{s c}\left(\Gamma^{\mu} \sum_{\left.\vec{x}_{s c} \mathrm{D}\left(0, x_{s c} ; \mathcal{U}\right) \mathrm{S}\left(x_{s c}, 0 ; \mathcal{U}\right)\right)}\right.
\end{aligned}
$$

where $\Gamma^{\mu} \otimes \Gamma^{\mu}=-\left(V^{\mu} \otimes A^{\mu}+A^{\mu} \otimes V^{\mu}\right)$.

From Eq. (4.7.18) it then follows that "at rest"

$$
\operatorname{fig}_{8 s c a l a r}\left(t_{s c}, 0, t_{D} ; \mathcal{U}^{P}\right)=\text { fig8scalar }_{2}\left(t_{s c}, 0, t_{D} ; \mathcal{U}\right)
$$

so that no new information is forthcoming by including the set $\left\{\mathcal{U}^{P}\right\}$ in the configuration average.

As in the previous cases, for "at rest" amplitudes

$$
\text { fig8scalar }_{2}\left(t_{s c}, 0, t_{D} ; \mathcal{U}^{C}\right)=\text { fig8scalar }_{2}\left(t_{s c}, 0, t_{D} ; \mathcal{U}\right)^{*}
$$

as a consequence of the identity Eq. (4.7.21). Therefore, only the real part of the function is retained. 
For the configuration $\mathcal{U}^{T}$ the correlation function becomes

$$
\text { fig8 }_{\text {calar }}\left(t_{s c}, 0, t_{D} ; \mathcal{U}^{T}\right)=\text { fig8scalar }_{2}\left(-t_{s c}, 0,-t_{D} ; \mathcal{U}\right)
$$

using identity Eq. (4.7.25).

Combining the results above the fig8scalar ${ }_{2}$ function is computed according to Eq. (4.7.41) but with the replacement

$$
\begin{aligned}
& \text { fig8scalar }_{2}\left(t_{s c}, 0, t_{D} ; \mathcal{U}\right) \rightarrow \\
& \qquad \begin{array}{r}
\frac{1}{2} \operatorname{Re}\left[\operatorname{fig} 8 s c a l a r_{2}\left(t_{s c}, 0, t_{D} ; \mathcal{U}\right)+\right. \\
\left.\quad \text { fig8scalar }_{2}\left(-t_{s c}, 0,-t_{D} ; \mathcal{U}\right)\right]
\end{array}
\end{aligned}
$$

\subsection{Continuum Functions}

The correlation functions that have been discussed so far have been lattice correlation functions that are computed using lattice operators within the lattice regularization scheme. The results of chapter 3 must now be applied to express the lattice results within the continuum renormalization scheme.

\subsubsection{Continuum Matrix Elements}

According to chapter 3 the matching condition between lattice operators and continuum operators is given by Eq. (3.3.6). For the $D \rightarrow K \pi$ decay modes this general relation was shown to reduce to

$$
\mathcal{O}_{ \pm}^{\text {cont }}(\mu)=\left[1+\frac{g^{2}}{16 \pi^{2}} Z_{ \pm}(r, a \mu)\right] \mathcal{O}_{ \pm}^{\text {lat }}
$$

where the perturbative matching coefficients $Z_{ \pm}(r, a \mu)$ are detined in Eq. (3.3.7). The "off-diagonal" operators $\mathcal{O}_{ \pm}^{\prime}$ do not contribute to the $D \rightarrow K \pi$ modes (at least 
to order $g^{2}$ ) since only parity changing operators will contribute to these modes. The lower-dimensional operators $\mathcal{O}_{i}^{(d<6)}$ were also shown not to contribute to these modes.

The linear combinations of operators

$$
\mathcal{O}_{ \pm}^{\text {lat }}=\frac{1}{2}\left[\mathcal{O}_{1}^{\text {lat }} \pm \mathcal{O}_{2}^{\text {lat }}\right]
$$

are the combinations that are multiplicatively renormalized. A lattice matrix element $\left\langle K \pi\left|\mathcal{O}_{ \pm}\right| D\right\rangle^{\text {lat }}$ is extracted by starting with the plus or minus linear combination of the four-point functions that will form the equivalent matrix element of the plus or minus operator combination. The procedure described in section 4.4 is then used to extract the matrix element from the TI ratio. These matrix elements can then be matched back to the continuum according to

$$
\left\langle K \pi\left|\mathcal{O}_{ \pm}\right| D\right\rangle^{\text {cont }}=\mathcal{Z}_{ \pm}\left\langle K \pi\left|\mathcal{O}_{ \pm}\right| D\right\rangle^{\text {lat }}
$$

where according to Eq. (4.8.1)

$$
\mathcal{Z}_{ \pm}=\left[1+\frac{g^{2}}{16 \pi^{2}} Z_{ \pm}(r, a \mu)\right]
$$

Putting in the dimensionful factors according to Eq. (4.6.3), and remembering Eq. (3.3.13) the continuum matrix elements are then related to the lattice matrix elements by

$$
\begin{aligned}
& \left\langle K \pi\left|\mathcal{H}^{e f f}\right| D\right\rangle^{c o n t}=\frac{G_{f} V_{c s}^{*} V_{u d} \sqrt{16 \kappa_{u, d}^{2} \kappa_{s} \kappa_{c}}}{2 \sqrt{2 a^{3}}} \\
& \left\{c_{+} \mathcal{Z}_{+}\left\langle K \pi\left|\mathcal{O}_{+}\right| D\right\rangle^{l a t}+c_{-} \mathcal{Z}_{-}\left\langle K \pi\left|\mathcal{O}_{-}\right| D\right\rangle^{l a t}\right\}
\end{aligned}
$$

where the continuum coefficients are computed with the effective number of flavors $N_{f}$ equal to zero to be consistent with the lattice quenched approximation. 
To discuss the $1 / \mathrm{N}$ behavior of the continuum diagrams the continuum matrix elements can be written in terms of the continuum $\mathcal{O}_{1}$ and $\mathcal{O}_{2}$ operators

$$
\begin{aligned}
& \left\langle K \pi\left|\mathcal{H}^{e f f}\right| D\right\rangle^{\text {cont }}=\frac{G_{f} V_{c s}^{*} V_{u d}}{2 \sqrt{2}} \\
& \left\{c_{1}\left\langle K \pi\left|\mathcal{O}_{1}\right| D\right\rangle^{\text {cont }}+c_{2}\left\langle K \pi\left|\mathcal{O}_{2}\right| D\right\rangle^{\text {cont }}\right\}
\end{aligned}
$$

with the quenched continuum coefficients

$$
\begin{aligned}
& c_{1}=\frac{1}{2}\left(c_{+}+c_{-}\right) \\
& c_{2}=\frac{1}{2}\left(c_{+}-c_{-}\right) .
\end{aligned}
$$

The lattice $\mathcal{O}_{1}$ and $\mathcal{O}_{2}$ operators are not diagonally renormalizable. The relationship between lattice and continuum matrix elements is then

$$
\begin{aligned}
& \left(\begin{array}{c}
\left\langle K \pi\left|\mathcal{O}_{1}\right| D\right\rangle \\
\left\langle K \pi\left|\mathcal{O}_{2}\right| D\right\rangle
\end{array}\right)^{\text {cont }}=\frac{\sqrt{16 \kappa_{u, d}^{2} \kappa_{s} \kappa_{c}}}{a^{3}} \\
& \left(\begin{array}{cc}
1+\frac{g^{2}}{16 \pi^{2}} Z_{11} & \frac{g^{2}}{16 \pi^{2}} Z_{12} \\
\frac{g^{2}}{16 \pi^{2}} Z_{21} & 1+\frac{g^{2}}{16 \pi^{2}} Z_{22}
\end{array}\right)\left(\begin{array}{c}
\left\langle K \pi\left|\mathcal{O}_{1}\right| D\right\rangle \\
\left\langle K \pi\left|\mathcal{O}_{2}\right| D\right\rangle
\end{array}\right)^{\text {lat }}
\end{aligned}
$$

where from Eq. (4.8.4)

$$
\begin{aligned}
& Z_{11}=Z_{22}=\frac{1}{2}\left(Z_{+}+Z_{-}\right) \\
& Z_{12}=Z_{21}=\frac{1}{2}\left(Z_{+}-Z_{-}\right)
\end{aligned}
$$

Note that the leading order large $\mathrm{N}$ behavior of the lattice and the continuum matrix elements are the same since the order $g^{2}$ corrections corresponding to the exchange of a single gluon which costs a factor of $1 / \mathrm{N}$. 


\subsubsection{Vacuum Saturation}

Vacuum saturation is implemented at the level of the continuum matrix elements rather than at the level of the lattice matrix elements. This is important since the goal is to test vacuum saturation on the continuum renormalized matrix elements. The matching condition is then identical to Eq. (4.8.3)

$$
\left\langle K \pi\left|\mathcal{O}_{ \pm}\right| D\right\rangle^{\text {cont }}=\mathcal{Z}_{ \pm}\left\langle K \pi\left|\mathcal{O}_{ \pm}\right| D\right\rangle^{\text {lat }}
$$

with $\mathcal{Z}_{ \pm}$defined in Eq. (4.8.4). The continuum vacuum saturation amplitudes can also be decomposed into matrix elements of the continuum $\mathcal{O}_{1}$ and $\mathcal{O}_{2}$ operators in exactly the same manner as the steps leading to Eq. (4.8.8). 


\section{Chapter 5. \\ Results}

\subsection{Lattices}

Numerical studies reported here were carried out on two independent $\beta=6.0$ lattices of dimensions $16^{3} \times 39$ and $24^{3} \times 39$ and on a $\beta=5.7$ lattice of dimension $16^{3} \times 33$. Earlier results were also reported for $\beta=5.7$ on a $16^{3} \times 25$ lattice and for $\beta=6.1$ on a $12^{3} \times 33$ lattice ${ }^{36-37}$. The parameters of these lattices are shown in table 5.1.1.

\begin{tabular}{|c|c|c|c|c|}
\hline$\beta$ & $a^{-1}(\mathrm{GeV})$ & lattice & length $(\mathrm{fm})$ & conf \\
\hline 6.0 & 2.0 & $\begin{array}{c}24^{3} \times 39 \\
16^{3} \times 39\end{array}$ & $\begin{array}{l}2.4 \\
1.6\end{array}$ & $\begin{array}{c}8 \\
19\end{array}$ \\
\hline 5.7 & 1.4 & $\begin{array}{l}16^{3} \times 33 \\
16^{3} \times 25\end{array}$ & 2.2 & $\begin{array}{c}16 \\
16\end{array}$ \\
\hline 6.1 & 2.2 & $12^{3} \times 33$ & 1.1 & 30 \\
\hline
\end{tabular}

Table 5.1.1: Properties of the various available lattices.

Results on the $\beta=5.716^{3} \times 25$ lattice are not included here because of concerns that this lattice is has too few time-slices sufficiently far from the location of the weak operator as to qualify as the asymptotic region (empirically $|t|>7$ for this lattice) and yet also be sufficiently far ( 3 to 4 time-slices) from the lattice time boundary. A large number of time-slices meeting the criteria above are necessary to perform multi-parameter fits as a function of time for quantities of interest. 
The $\beta=6.1$ results are not included since the physical size of this lattice is small and again the number of "asymptotic" time-slices is limited. Previously" it was shown that while the $\beta=6.1$ results are not inconsistent with results from other lattices, they tend to have much larger finite size effects, which reduces their significance.

The inverse lattice spacing shown in table 5.1.1 for $\beta=6.0$ lies in the middle of the range of values found from string tension measurements ${ }^{38,36}$. Values for the lattice spacing at other values of $\beta$ are determined using the $\beta=6.0$ value and assuming scaling relation Eq. (3.3.15) holds over the region of $\beta$ values considered. Previous studies have shown some evidence for a lack of scaling between $\beta=6.0$ results and $\beta=5.7$ results. Thus, the lattice spacing quoted here for $\beta=5.7$ is at best an estimate.

These inverse lattice spacings are different than the lattice spacings previously used $^{36}$. Those previously used values were considered to have a precision of about $\pm 20 \%$. Since string tension measurements are still rather difficult to perform, the new value at $\beta=6.0$ may still have an uncertainty of about $\pm 20 \%$.

Table 5.1.2 shows the combinations of hopping constants used in this study for the $\beta=6.0$ and $\beta=5.7$ lattices. Computations were performed with quark propagators with the tabulated hopping constant values at each beta for both the exact flavor $S U(2)$ case (exact isospin symmetry case) and for the exact flavor $S U(3)$ case. The flavor $S U(3)$ case affords a comparison with continuum models in which $S^{\prime} U(3)$ flavor symmetry allows a considerable simplification in formulae.

The hopping constants $\kappa_{u, d}=.155$ and .166 correspond to quark mass ratios $m_{\mathrm{u}, \mathrm{d}} / m_{\mathrm{s}}$ of about 0.42 and 0.34 for $\beta=6.0$ and 5.7 respectively. For quarks much lighter than these the cost in terms of numerical effort increases greatly. 


\begin{tabular}{|c|c|l|l|l|}
\hline$\beta$ & description & $\kappa_{u, d}$ & $\kappa_{s}$ & $\kappa_{c}$ \\
\hline 6.0 & $S U(2)_{f}$ & $\begin{array}{l}.155 \\
.154\end{array}$ & .152 & .118 \\
\hline 6.0 & $S U(3)_{f}$ & \multicolumn{2}{|c|}{$\begin{array}{l}.155 \\
.154\end{array}$} & .118 \\
& & \multicolumn{2}{|c|}{.152} & \\
\hline 0.0 & lighter c quark & .155 & .152 & .135 \\
\hline 5.7 & $S U(2)_{f}$ & $\begin{array}{l}.166 \\
.164\end{array}$ & .160 & .094 \\
\hline 5.7 & $S U(3)_{f}$ & \multicolumn{2}{|c|}{$\begin{array}{l}.166 \\
.164\end{array}$} & .094 \\
\hline
\end{tabular}

Table 5.1.2: Hopping constants used for each $\beta$ value. The $\kappa$ value at which the $\overline{\mathrm{q}} \mathrm{q}$ pseudoscalar bound state becomes massless is $\kappa_{c r i t}=$ $.1573(2)$ at $\beta=6.0$ and $\kappa_{\text {crit }}=.1693(1)$ at $\beta=5.7$.

The choice $\kappa_{c}=.135$ results in a lighter $\mathrm{c}$ quark and is the $\kappa_{c}$ value used by the ELC group $^{39}$. The value $\kappa_{c}=.118$ used here seems to give a charm meson that is heavier than the experimental charm meson mass. Because this is still a preliminary study of $D$ decays we are not concerned with tuning the s and c quark masses to more precise values.

\subsection{Propagators and $\chi^{2}$ Fits}

Pseudoscalar and scalar propagators were calculated as described in sections 4.2 and 4.3. Figures I.1.1 through I.4.1 show examples of the time dependence of the $\vec{p}=\overrightarrow{0}$ pseudoscalar and scalar propagators for the combined $\beta=6.0$ lattices.

To combine two independent lattice 3 as is done in this study for the two $\beta=6.0$ lattices, the configuration average of a quantity is taken over the joint set of 
configurations for both lattices. Each lattice can also be analyzed separately to check for systematic effects such as finite size effects.

Chi-square fits were performed taking into account correlations between the fitted points. The error bars shown for each time-slice $t_{i}$ are given by $\pm \sqrt{\sigma\left(t_{i}, t_{i}\right)}$ where the data covariance matrix $\sigma\left(t_{i}, t_{j}\right)$ is computed according to

$$
\sigma\left(t_{i}, t_{j}\right)=\frac{1}{N(N-1)} \sum_{k=1}^{N}\left[v_{k}\left(t_{i}\right)-\bar{v}\left(t_{i}\right)\right]\left[v_{k}\left(t_{j}\right)-\bar{v}\left(t_{j}\right)\right]
$$

The index $k$ runs over configurations and the quantity $v_{k}\left(t_{i}\right)$ is the value of the (pseudo)scalar propagator calculated at time-slice $t_{i}$ in the fixed gauge cc $r$ figuration labeled $k$. The quantity $\bar{v}\left(t_{i}\right)$ is the configuration average of the $v_{k}\left(t_{i}\right)$

$$
\bar{v}\left(t_{i}\right)=\frac{1}{N} \sum_{k=1}^{N} v_{k}\left(t_{i}\right)
$$

The fits to the calculated propagator values shown in these graphs are all of the functional form

$$
F(t ; \vec{a})=\sum_{r=1}^{n} \frac{Z_{r}^{2}}{2 m_{r}} e^{-m_{r} t}
$$

where $\vec{a}=\left(Z_{1}, m_{1}, \ldots, Z_{n}, m_{n}\right)$ is the parameter vector. A sum over exponentials is used here to allow for possible contamination of a propagator by states that are radial excitations of the ground state.

The fits are obtained by varying $\vec{a}$ so as to minimize the formal chi-square function

$$
\chi^{2}(\vec{a})=\sum_{t_{i}, t_{j}}\left[\bar{v}\left(t_{i}\right)-F\left(t_{i} ; \vec{a}\right)\right] \sigma^{-1}\left(t_{i}, t_{j}\right)\left[\bar{v}\left(t_{j}\right)-F\left(t_{j} ; \vec{a}\right)\right]
$$

where $t_{a} \leq t_{i}, t_{j} \leq t_{b}$ and $t_{a}$ is the lower bound of the fitted interval and the upper bound is $t_{b}$. 


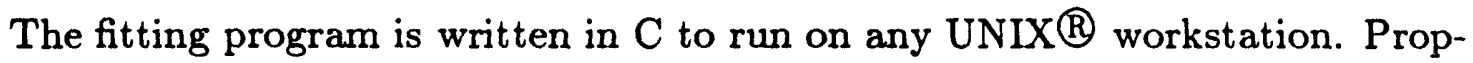
agators as a function of time-slice and background gauge configuration are computed from information stored in the "condensed" files by an analysis program that runs on Cray supercomputers. This intermediate analysis program then converts floating-point numbers in Cray format to the IEEE Double representation which is the internal data representation used on a large number of workstations and writes this information to a file. These binary data files are then transferred via network connections to a local workstation for storage and final analysis. Currently the format of these binary files is specialized to this analysis. However, there is a standard package of routines called Hierarchical Data Format ${ }^{40}$ (HDF) that allows scientific data sets and raster images to be read and written by a wide range of supercomputers and workstations. Using HDF the fitting program could be modified to accept general data sets.

The program uses the Levenberg-Marquardt algorithm as described by Press ${ }^{41}$ et al. with some modifications. The algorithm was modified to use the full covariance matrix $\sigma\left(t_{i}, t_{j}\right)$ rather than assuming statistically independent errors as was treated in the reference. Additionally, the Gauss-Jordan matrix inverter used internally in the original program was replaced by the more robust Singular Value Decomposition method. This method of matrix inversion is capable of detecting ill-conditioned matrices.

The fits generated by this program were checked against fits generated by minimizing the function Eq. (5.2.4) in Mathematica ${ }^{\circledR}$.

Fits to the propagators were performed using only one exponential term. The results of these fits for each of the $\beta=6.0$ lattices and the two lattices in combination are shown in Tables I.1.1 through I.4.1. 
The estimated coefficient of covariance for the fitted parameters is given along with the formal $\chi_{\min }^{2}$ per degree of freedom for the fit. The coefficient of covariance $c_{a b}$ is given by the relation

$$
c_{a b}=\frac{C_{o v} v_{a b}}{\sqrt{C_{o v a b} C o v_{b b}}}
$$

where $C_{o v_{a b}}$ is an element of the parameter covariance matrix. The quoted error bound $\delta a_{b}$ on a fitted parameter $a_{b}$ is given by

$$
\delta a_{b}= \pm \sqrt{\operatorname{Cov}_{b b}}
$$

This corresponds to the $\Delta \chi^{2}=1$ contour of constant $\chi^{2}$. The contours of constant $\chi^{2}$ may be estimated from

$$
\Delta \chi^{2}=\chi^{2}-\chi_{\min }^{2}=\delta \vec{a} \cdot \operatorname{Cov}^{-1} \cdot \delta \vec{a}
$$

or they may be found directly by making a contour plot of $\chi^{2}(\vec{a})$ in Eq. $(5.2 .4)$ using a program such as Mathematica.

Since the data values $v_{k}\left(t_{i}\right)$ for the propagators and also for nearly every other measured quantity in lattice gauge theory calculations are not normally distributed it is important to note that the quoted error bounds as given by Eq. (5.2.6) are not the standard $68 \%$ confidence interval as would be the case for $\Delta \chi^{2}=1$ and normally distributed errors. To determine which $\Delta \chi^{2}$ to use when quoting standard confidence levels would require additional calculation.

For the pion, kaon, and the $D$ the fitting interval on the $\beta=6.0$ lattices was taken to be the range $[12,16]$. In physical units this corresponds to $1.2 \lessgtr t \lesssim 1.6 \mathrm{fm}$ while staying three time-slices away from the boundary. 
The formal $\chi^{2}$ per degree of freedom for these fits range from about $.2 / 3$ to about $6 / 3$. Taking this as a relative measure of the goodness of fit, the best fits are obtained for the pion and kaon while the fits to the $D$ propagators are markedly worse.

One possible reason for the higher $\chi^{2} / n_{d}$ in the $D$ case may be that a radial excitation of the $D$ may have a relatively small difference in energy compared to the $D$. Contamination of the propagator by this excited state may still be present in the time range fitted.

To test this argument one can select a model function with more than one exponential term. Take $\kappa_{u, d}=.152, \kappa_{c}=.118$ on the combined 6.0 lattices. A two exponential fit on the time interval $[6,16]$ gives the results in the first row of the table below. For comparison, the third row shows the one exponential fit.

\begin{tabular}{|c|c|c|c|c|c|}
\hline te.ms & $Z_{1}$ & $m_{1}$ & $Z_{2}$ & $m_{2}$ & $\frac{\chi^{2}}{n_{d}}$ \\
\hline 2 & $.913(34)$ & $1.232(5)$ & $2.79(52)$ & $1.78(8)$ & $\frac{8.6}{7}$ \\
\hline 2 & $.894(32)$ & $1.231(5)$ & $2.79^{*}$ & $1.78^{*}$ & $\frac{6.0}{3}$ \\
\hline 1 & $.915(32)$ & $1.233(5)$ & - & - & $\frac{6.0}{3}$ \\
\hline
\end{tabular}

The second row is a two exponential fit in the time range $[12,16]$ with the parameters of the second exponential fixed (indicated by * next to the values held fixed). Comparing to the third row one sees that stripping out the excited state makes no difference in the $\chi^{2}$. The same conclusion is reached by taking the ratio of the two exponential terms. This ratio is at most .04 at $t=12$ so the excited state above is a small contamination. As noted in Chapter 4 the contamination due to excited states is reduced by choosing interpolating fields that extend over more than a single lattice site. 


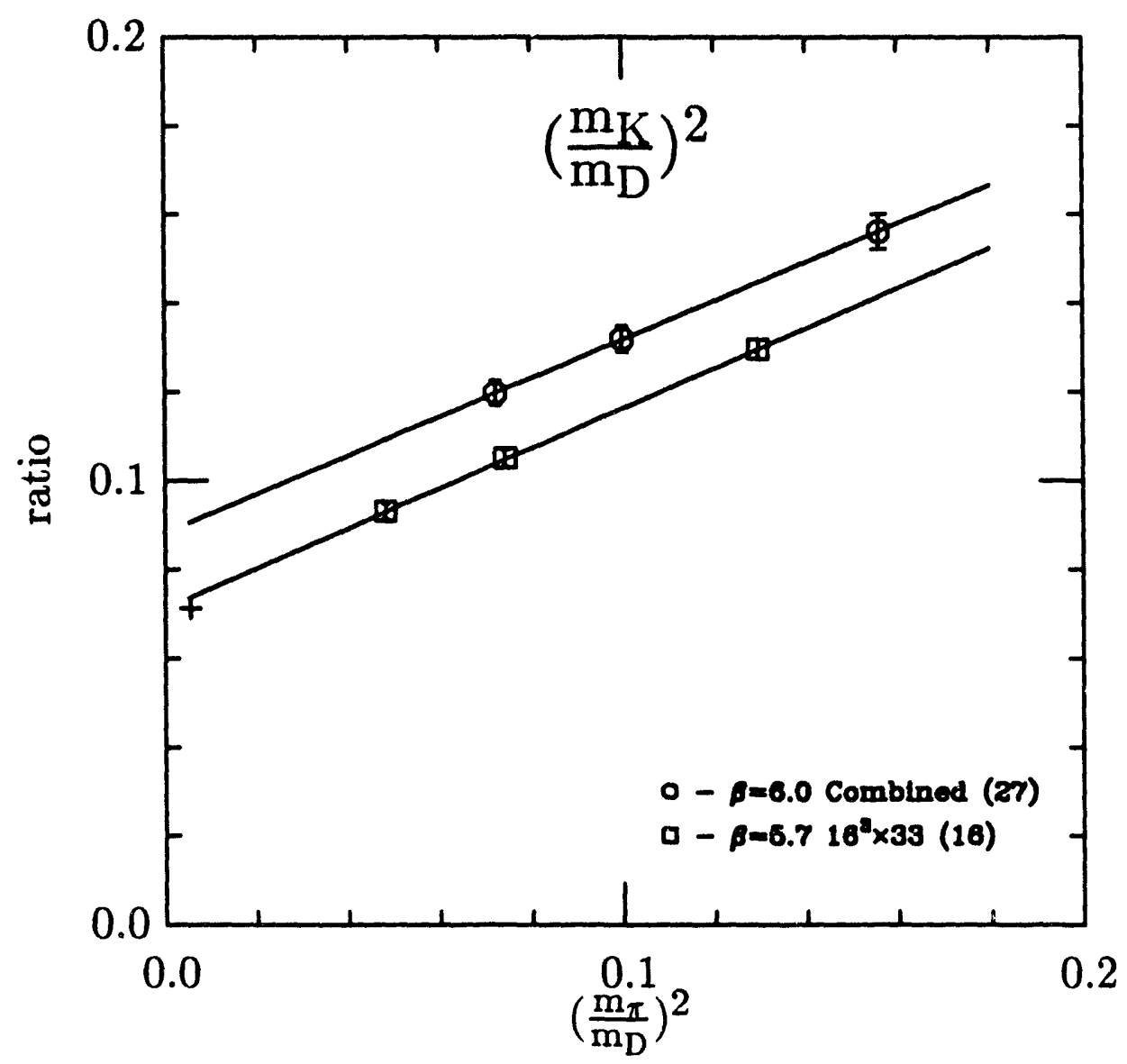

Figure 5.2.1: Graph of $\left(m_{K} / m_{D}\right)^{2}$ versus the ratio $\left(m_{\pi} / m_{D}\right)^{2}$. The lines do not take into account correlations between plotted points. The cross on the left side of the graph is the physical point.

In figure 5.2.1 the ratio $m_{K}^{2} / m_{D}^{2}$ versus the ratio $m_{\pi / m_{D}^{2}}^{2}$ is plotted. The ratios of pseudoscalar masses are taken since according to chiral perturbation theory the mass squared of the pion and kaon depend linearly on the valence quark masses. Then assuming that the $D$ meson mass varies little with the light quark mass one concludes the relation between the plotted quantities is linear.

The point marked with a cross is calculated from the physical masses. The charm and strange hopping constants can be adjusted so that the extrapolated 


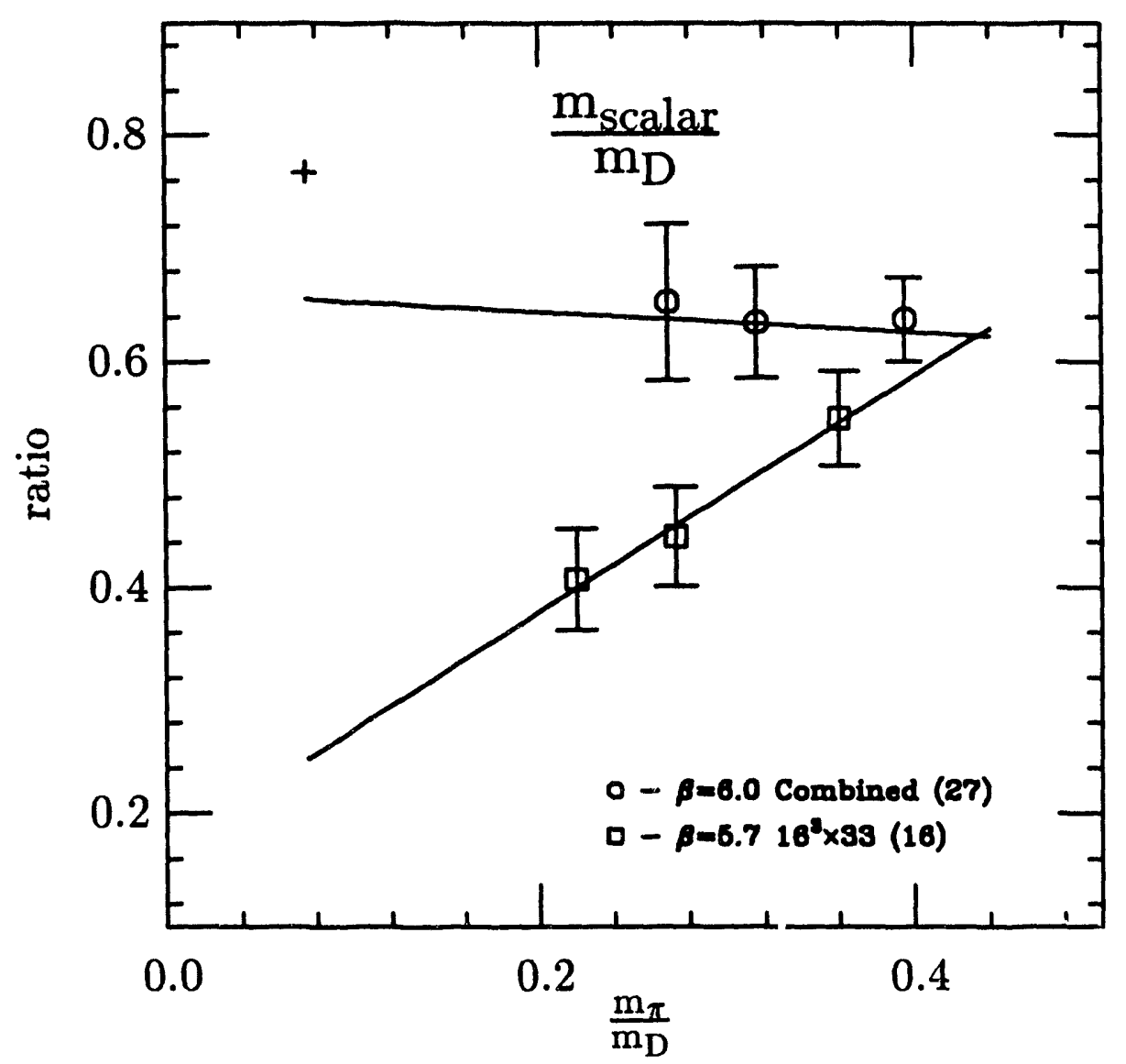

Figure 5.2.2: Graph of $m_{\text {scalar }} / m_{D}$ vs $m_{x} / m_{D}$. The lines do not take into account correlations between plotted points.

curve passes through the physical point when the pion mass is set equal to the physical value.

Next examine the fits to the scalar propagator in table I.4.1 and the sample fit shown in figure I.4.1. The error bars shown in the figure become very large for time-slices greater than about ten. Neglecting relativistic effects, the wave function for the scalar $(L=1)$ state is expected to vanish at the origin. The scalar state then couples through relativistic effects to the point-like interpolating fields used here. Using an interpolating field for the scalar with a spatial size larger than a point should improve the coupling to the scalar state. 
Single exponential fits to the scalar propagator propagator were performed in the time range $[9,14]$ for $\beta=6.0$ and for the range $[6,12]$ on the $\beta=5.7$ lattice. Figure 5.2.2 shows the ratio of the fitted scalar mass to the $D$ mass versus the pion mass scaled by the $D$ mass. If the scalar state is identified with the physical state $\left(0^{+}\right)$state labeled $K_{0}^{*}(1430)$ in the Particle Data Table ${ }^{3}$, then the physical point is the cross shown in the upper left of the figure. Note the apparent discrepancy between the 6.0 points and the 5.7 points. Computing the mass spectrum of scalar states has proved to be more difficult than computing the spectrum of pseudoscalar states. Perhaps the spectroscopy of scalar states must await bigger lattices and a choice for interpolating fields that couple better to the scalar state.

In this project the main concern with the scalar is potential off-shell contributions to $D \rightarrow K \pi$ matrix elements it may make as in Eq. (4.4.23). As that equation shows, off-shell contribution depends on the quantity

$$
\delta m=-\left(m_{\text {scalar }}-m_{K}-m_{\pi}\right) .
$$

The quantity $\delta m$ can be computed by fitting the time dependence of the ratio

$$
\frac{G_{s c}^{(2)}(t, 0)}{G_{K}^{(2)}(t, 0) G_{\pi}^{(2)}(t, 0)}
$$

to the functional form $c_{0} e^{\delta m t}$ which is expected from the asymptotic behavior of the propagators.

The $\delta m$ values from fits for $\beta=6.0$ are shown in table I.5.1 while figure I.5.1 is an example of such a fit. From the fits it is hard to extract even a rough value for the scalar mass. However, these fits do show that the scalar mass is close enough to $m_{\pi}+m_{K}$ that the scalar may make an important off-shell contribution to $D \rightarrow K \pi$ matrix elements. 


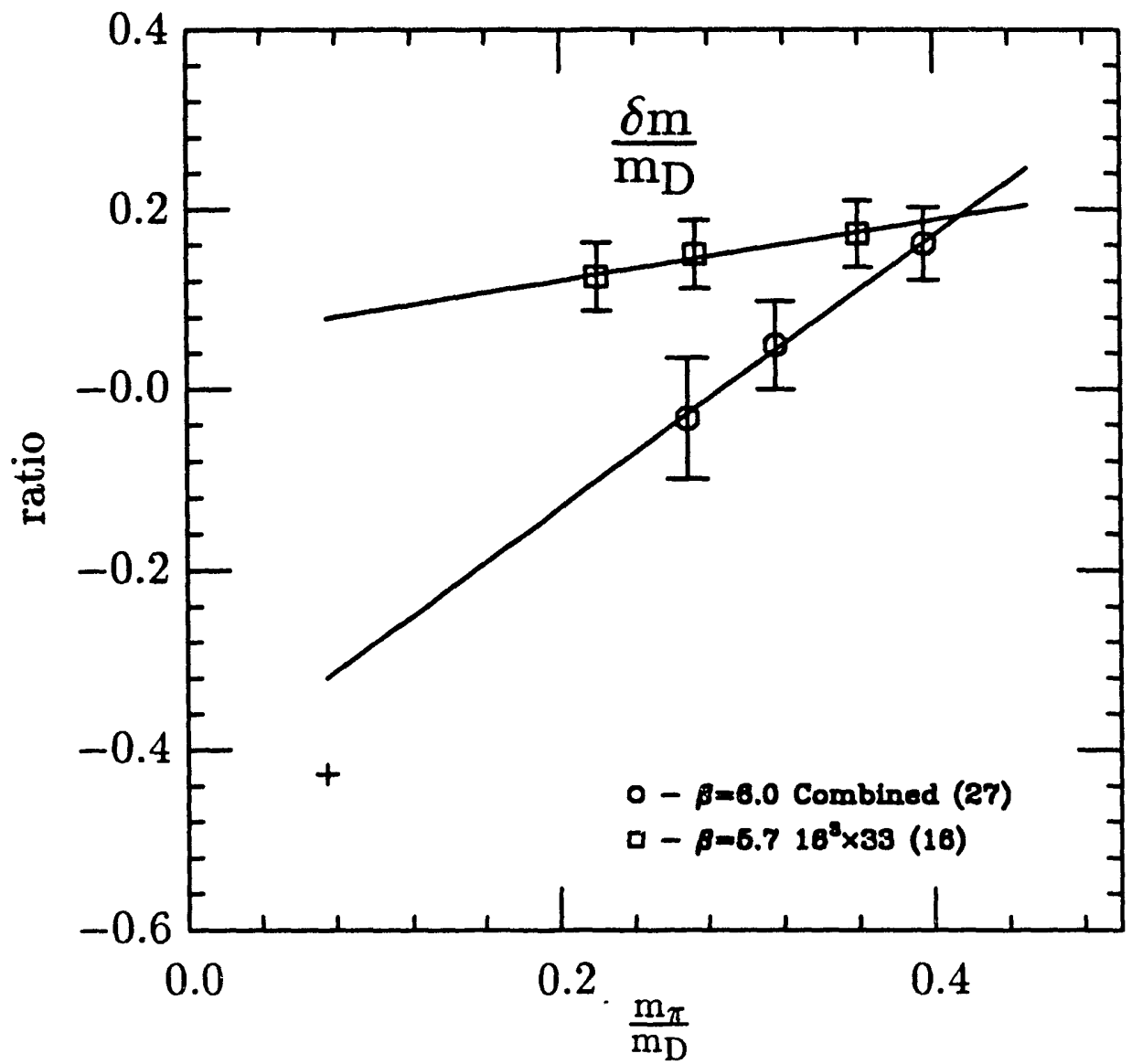

Figure 5.2.3: The quantity $\delta m / m_{D}$ versus $m_{\pi} / m_{D}$. All quantities are scaled by the $D$ mass. The two lines do not take into account correlations between plotted points.

The error bars in figure I.5.1 and the data covariance matrix used in the $\chi^{2}$ fitting procedure used to fit the ratio of propagators were calculated using the jackknife procedure ${ }^{42}$.

A synthetic or jackknife data value is generated for a quantity by omitting one data sample i.e. one configuration when calculating the quantity. A set of jackknife data values is then created by repeating the process for each configuration. 
Hence, jackknife data values for a propagat,or are calculated according to

$$
v_{k}^{\prime}\left(t_{i}\right)=\frac{1}{N-1} \sum_{\substack{j=1 \\ j \neq k}}^{N} v_{j}\left(t_{i}\right)
$$

where $v_{k}^{\prime}\left(t_{i}\right)$ is jackknife data value for the propagator that results by omitting the $k^{\text {th }}$ configuration from the average and $v_{j}\left(t_{i}\right)$ is the propagator with background gauge field configuration $j$.

For the ratio of propagators in Eq. (5.2.9), calculate jackknife data values $r_{k}^{\prime}(t)$ according to

$$
r_{k}^{\prime}\left(t_{i}\right)=\frac{G_{s c}^{(2) \prime}\left(t_{i}, 0\right)_{k}}{G_{K}^{(2) \prime}\left(t_{i}, 0\right)_{k} G_{\pi}^{(2) \prime}\left(t_{i}, 0\right)_{k}}
$$

where the propagators are calculated as in Eq. (5.2.10). The subscript $k$ once again indicates that the quantity is calculated omitting the $k^{\text {th }}$ configuration.

The covariance of these jackknife data values is then estimated from

$$
\sigma\left(t_{i}, t_{j}\right)=\frac{N-1}{N} \sum_{k=1}^{N}\left[r_{k}^{\prime}\left(t_{i}\right)-\bar{r}^{\prime}\left(t_{i}\right)\right]\left[r_{k}^{\prime}\left(t_{j}\right)-\bar{r}^{\prime}\left(t_{j}\right)\right]
$$

where

$$
\bar{r}^{\prime}\left(t_{i}\right)=\frac{1}{N} \sum_{k=1}^{N} r_{k}^{\prime}\left(t_{i}\right)
$$

is used as an unbiased estimator of the average. Note that this $\bar{r}^{\prime}\left(t_{i}\right)$ is used only to compute the statistic above. The value reported for the ratio is calculated from Eq. (5.2.9).

The jackknife data covariance matrix calculated above is used along with the averages for a given quantity as inputs to the $\chi^{2}$ minimization algorithms previously described to determine the "best" fits. 
Jackknife uncertainty estimates and the fitting procedure followed for the ratio of propagators is also used to perform fits to ratios of other quantities such as the time-independent (TI) ratio Eq. (4.4.22) from which matrix elements are extracted.

\subsection{Time Dependence of Amplitudes}

This discussion focuses upon the time-independent (TI) ratios (Sect. 4.4) calculated on the combined $\beta=6.0$ lattices. The $\beta=5.7$ results are not shown since they are qualitatively the same.

Figures 5.3.1 through 5.3.3 show the TI ratios for the $D \rightarrow K \pi$ figure eight correlation functions. The quantities shown in these figures must be multiplied by the propagator $\mathrm{Z}$ constants $Z_{\pi}, Z_{K}$, and $Z_{D}$ to match the definition of the TI ratio given by Eq. (4.4.22).

For the two $\beta=6.0$ lattices the weak operator is located at the center of the lattice $\left(t_{K}=0\right)$ and the lattice boundaries are located at $\left|t_{K}\right|=19$. Only the positive half of the lattice is shown in the figures since Euclidean $T$ symmetry (Sect. 4.7) is used to combine calculations from both sides of the lattice.

The correlation functions shown have the pion held fixed at $t_{\pi}=11$ and the $D$ held fixed at $t_{D}=-11$. The $t_{K}$ coordinate is then varied. To allow $t_{K}$ to be varied for all three types of fig 8 diagrams, the lattice four-point functions use exponentiated quark propagators with $t_{\exp }=t_{D}$ for fig8 8 and fig8K (see sect. II.4) and exponentiation at $t_{e x p}=t_{\pi}$ for fig8an. With this combination of exponentiations, the correlation functions obtained correspond to the diagrams of figure 4.5.1 with all of the quark lines reversed. 


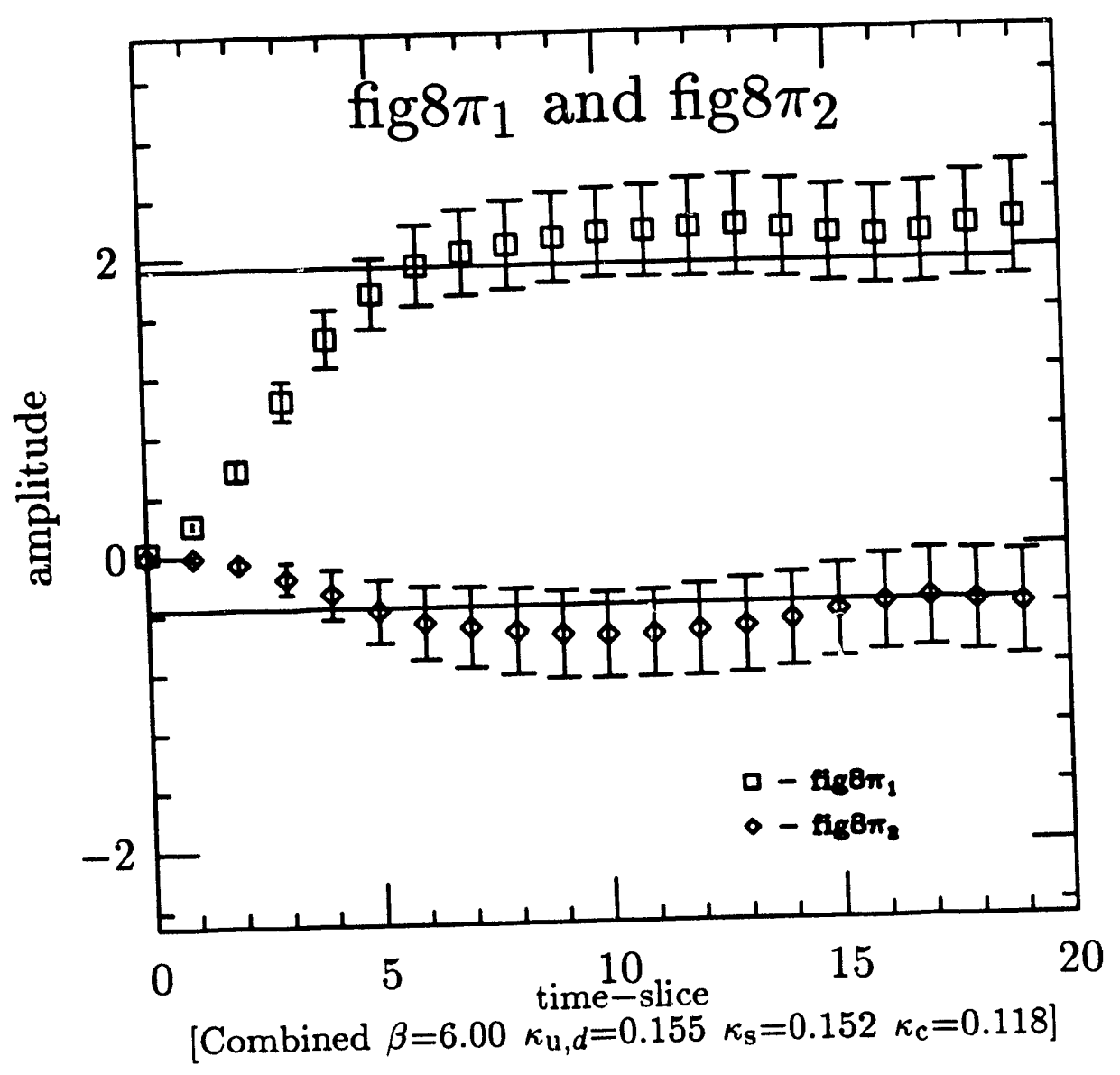

Figure 5.3.1: TI ratio for fig $8 \pi_{1}$ and fig $8 \pi_{2}$ versus $t_{K}$. The correlation functions are matched to the continuum.

The expressions for the correlation functions corresponding to the quark line directions as shown in figure 4.5.1 require exponentiations at $t_{K}$ for fig8 8 and fig8an and at $t_{\pi}$ for fig8K.

Regardless of which exponentiation is used to calculate a correlation function, for a given combination of $t_{D}, t_{K}$, and $t_{D}$, the same numerical value must be obtained since reversing quark lines serves only to replace each particle by its anti-particle. Each of the fig8 functions was calculated using the two different exponentiations described above. A check for numerical equality then served as a test of the exponentiated propagators. 


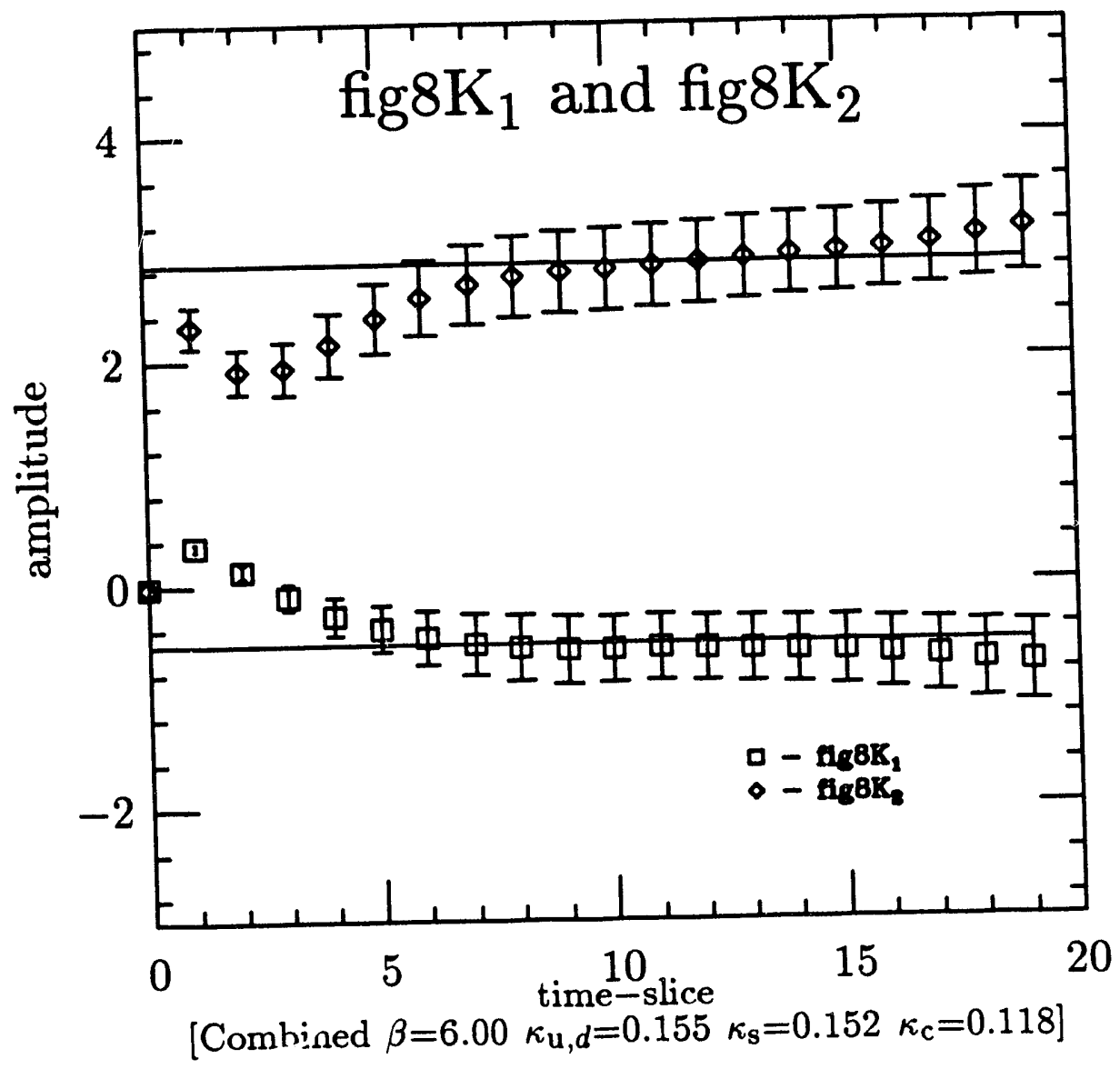

Figure 5.3.2: $\mathrm{TI}$ ratio for fig $8 \mathrm{~K}_{1}$ and fig $8 \mathrm{~K}_{2}$ versus $t_{K}$. The correlation functions are matched to the continuum.

Continuum operators $\mathcal{O}_{1}$ and $\mathcal{O}_{2}$ determined by perturbative matching to lattice operators as described in Sect. 3.3 are used in the calculation of these TI ratios. To obtain continuum matrix elements matching the definition in Eq. (4.8.8), requires multiplication by a factor of $a^{-3} \sqrt{16 \kappa_{u, d}^{2} \kappa_{s} \kappa_{c}}$ in addition to the $Z$ factors previously mentioned.

The fits shown in figures 5.3.1 through 5.3.3 are best fits of the correlation functions to the constant function over the time range $[12,16]$. A fit to a constant function neglects the effects of the time-dependent terms in Eq. (4.4.23). For the spectators one determines a naive plateau value that is within the jackknife error 


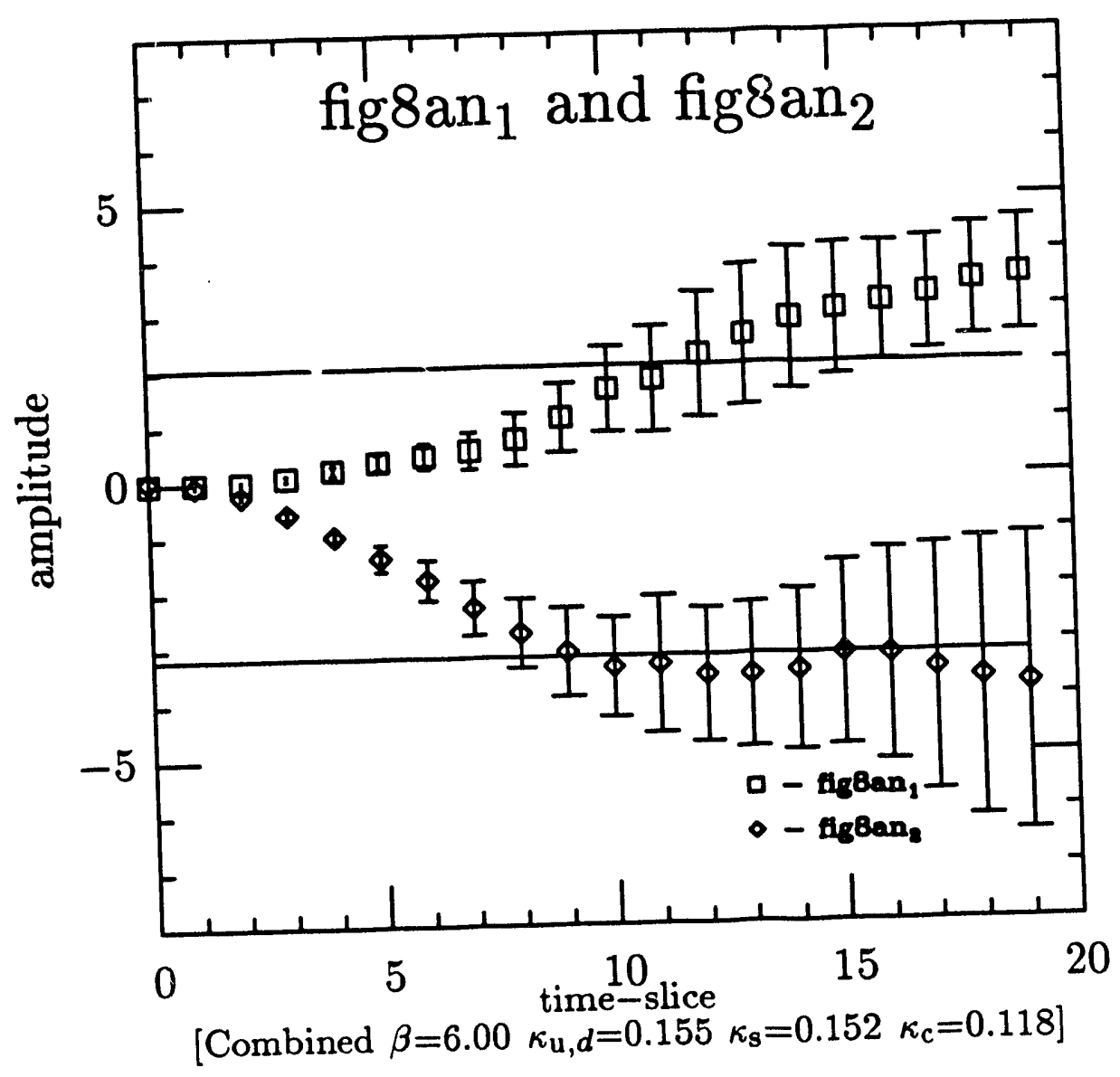

Figure 5.3.3: TI ratio for fig8an ${ }_{1}$ and fig8an 2 versus $t_{K}$. The correlation functions are matched to the continuum.

bars over the time range fitted. The formal $\chi^{2}$ per degree of freedom for the fits to the spectator functions are all less than $3 / 4$. Note that the best fit lines for the spectators lie consistently above or below the fitted points.

That a functional fit lies consistently above or below all of the central values should not be surprising. The central values on successive time-slices show almost no statistical fluctuation compared to what one might expect based on the size of the error bars shown and statistically independent measurements. This is a certain indication that the central values on successive time-slices are highly correlated. The error bars shown in the graphs give only the diagonal elements of a general 
correlation matrix (see Sect. 5.2). Taking into account the correlations among time-slices can result in fits which show an apparent bias to one side or the other of all of the fitted points.

Consider the annihilation functions in Fig. 5.3.3. The $\chi^{2}$ per degree of freedom is $7 / 4$ for fig8an 1 and $10 / 4$ for fig8an ${ }_{2}$. Here we see some indication for a time dependence in the TI ratio. Previous lattice studies ${ }^{23,43}$ of $K \rightarrow \pi \pi$ employing the direct calculation of four-point functions have seen similar evidence for time dependence in $\mathrm{TI}$ ratios. In $K$ decays this time dependence has been attributed to contributions due to the scalar $\sigma$ particle that can couple to two pions. Continuum studies $^{44}$ also suggest scalar resonances may play a role in $K \rightarrow \pi \pi$ decays.

The eye can discern a clear linear rise in the central values of the fig8an 1 TI ratio as a function of $t$. Returning to the spectators one may also discern such a trend in their graphs (see fig8 $\mathrm{K}_{2}$ in Fig. 5.3.2). Note that even for fig8an ${ }_{1}$, which seems to show the most obvious time dependence, the rise in the central values over the interval $[12,16]$ is less than one error bar.

Even though the total variation with time of the TI ratios is less than the error bars shown in the graphs, the time dependent terms responsible for this variation can be appreciable. We attempt to estimate the effects of time-dependent terms in the behavior of $\mathrm{TI}$ ratios by considering combinations of correlation functions with definite final state isospin. Multiply the $\mathcal{O}_{1}$ and $\mathcal{O}_{2}$ correlation functions by the Wilson coefficients Eq. (4.8.7) and form the linear combinations

$$
\begin{aligned}
G_{1 / 2}^{(4)} & =\sqrt{\frac{2}{3}}\left(\text { fig8 } 8 \pi-\frac{1}{2} \text { fig8K }+\frac{3}{2} \text { fig8an }\right) \\
G_{3 / 2}^{(4)} & =\sqrt{\frac{1}{3}}(\text { fig } 8 \pi+\text { fig8K })
\end{aligned}
$$

of final state isospin $1 / 2$ and $3 / 2$ respectively. 
Typical plots of the TI ratio for these quantities are shown in Fig. I.13.1 and I.16.1. From these figures we see that the TI ratio for $G_{3 / 2}$ shows a slight drop after time-slice eleven. The TI ratio for $G_{1 / 2}$ shows a rather dramatic linear rise with time. The fits shown in the graphs are again fits using the constant function as the model function. The details of these fits are shown in the accompanying tables found in the appendix.

Specializing Eq. (4.4.23) for the case $0 \ll t_{\pi} \ll t_{K}$ yields for the TI ratios of the quantities in Eq. (5.3.1)

$$
\begin{aligned}
\operatorname{TIR}_{1 / 2}=\mathcal{A}_{1 / 2}\left[1-\alpha_{1 / 2}\left(\frac{2 \mu}{\pi t_{K}}\right)^{1 / 2}+\ldots\right]+ \\
\mathcal{A}(D \rightarrow \kappa) \frac{2 m_{K} \mathcal{M}(\kappa \rightarrow K \pi)}{\delta m\left(\delta m-2 m_{K}\right)} e^{\delta m t_{K}} \\
\operatorname{TIR}_{3 / 2}=\alpha_{3 / 2}\left[1-\alpha_{3 / 2}\left(\frac{2 \mu}{\pi t_{K}}\right)^{1 / 2}+\ldots\right]
\end{aligned}
$$

where $\mathcal{A}_{I}$ is the "at rest" amplitude for isospin component $I$ and $\alpha_{I}$ is the corresponding scattering length for $K \pi$ scattering.

The term proportional to $\mathcal{A}(D \rightarrow \kappa)$ describes a possible contribution to the TI ratio by a $0^{+} \bar{d}$ s state which here is called $\kappa$. Such a scalar state might be expected to show the strongest signature in the weak annihilation function. The annihilation function has a pure isospin $1 / 2$ final state and the final state quark and antiquark emitted from the effective weak vertex are an $\mathrm{s}$ and $\mathrm{a} \overline{\mathrm{d}}$ respectively. In the figure below is shown the weak annihilation diagram for $D^{0} \rightarrow K^{-} \pi^{+}$. The dashed line represents some intermediate time before the creation of the final state u $\bar{u}$ pair. At such times we see a two quark intermediate state which can form a scalar bound state. 


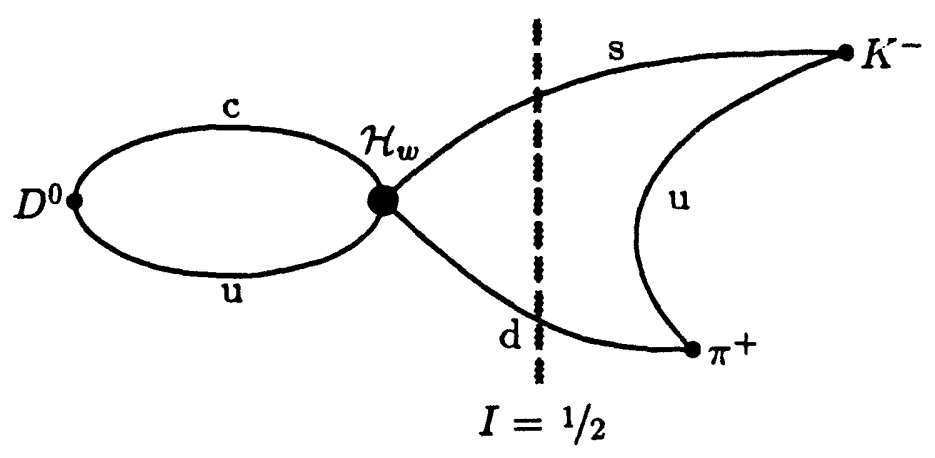

An indication of a $0^{+}$particle that couples to $K \pi$ has been found in an analysis of $K \pi$ scattering ${ }^{45}$. The particle is listed as $K_{0}^{*}(1430)$ in the particle data table ${ }^{3}$. The particle is a scalar with isospin $1 / 2$ and a decay width of $\Gamma=287 \pm 23 \mathrm{MeV}$. It decays predominantly to $K \pi$. With a mass of $1.43 \mathrm{GeV}$ and a relatively wide decay width, this resonance is potentially important to $D^{0}$ decay.

It must be emphasized that any physical contribution of this resonance to $D^{0}$ decay is already accounted for in the $\mathcal{A}_{1 / 2}$ amplitude of Eq. (5.3.2a). The term proportional to $\mathcal{A}(D \rightarrow \kappa)$ is an unphysical contribution that is not suppressed in Euclidean space (see Sect. 4.4).

Numerical results were obtained for the quantity $\delta m$ as discussed in Sect. 5.2. The results there are still rather crude. From table I.5.1 one finds $\delta m=-.038 \pm$ .079 for the combined $\beta=6.0$ lattices and $\kappa_{u, d}=.155$. For $\beta=6.0$ the general trend found for $\delta m$ is promising. Note that according to Fig. 5.2.3 $\delta m$ increases and becomes negative as the pion mass is decreased. From Eq. (\$.3.2) we see that the off-shell term will be exponentially suppressed. With a larger physical lattice time range and smaller light quark masses the relative importance of this term may diminish.

We would like to make an estimate of the size of the scalar contamination. First, we must estimate the magnitude of the off-shell quantity $\mathcal{M}(\kappa \rightarrow K \pi)$. We approximate it by using the on-shell invariant amplitude calculated from the 
experimental decay width. From the width and mass quoted above for $K_{0}^{*}$ and assuming the branching fraction is $100 \%$, one finds $\mathcal{M}(\kappa \rightarrow K \pi)_{\text {on-shell }} \approx 4.9 \mathrm{GeV}$. Taking a nominal value of $a^{-1}=2.0 \mathrm{GeV}$ and assuming scaling holds between the continuum and the lattice, one finds $\mathcal{M}(\kappa \rightarrow K \pi) \approx 2.4$ as a crude estimate of the quantity in Eq. (5.3.2a) measured in lattice units. Based on the success of measuring other hadronic coupling constants ${ }^{46}$, we note that it should be possible to estimate this quantity by direct lattice calculation.

The quantity $\mathcal{A}(D \rightarrow \kappa)$ in Eq. (5.3.2a) was calculated on the lattice in this study and an example of these results are found in Sect. I.21. For the combined lattices taking $\kappa_{u, d}=.155$ one finds $\mathcal{A}(D \rightarrow \kappa)=.07 \pm .04$.

The estimates above combine to give approximately 4 as an order of magnitude estimate of the prefactor of the exponential term in Eq. (5.3.2a). Thus at time slice $t_{K}=14$ the exponential term is estimated to be about 2.5 in lattice units. This is to be compared with the graph for the TI ratio for $G_{1 / 2}$ shown in Fig. I.16.1 which gives a value of about 4.2 at $t_{K}=14$. The exponential term in Eq. (5.3.2a) can potentially be a significant fraction of the TI ratio for $G_{1 / 2}$.

Next we attempt an order of magnitude estimate of the terms proportional to the scattering lengths $\alpha_{I}$ in Eq. (5.3.2). The tree level expressions for the scattering lengths calculated in chiral perturbation theory are given in Eq. (4.4.24). The pion decay constant has been measured on the lattice ${ }^{47}$. Based on these measurements, the pion decay constant for $\beta=6.0$ with $\kappa_{u, d}=.155$ is $f_{\pi} \approx .07$ in lattice units. Approximate values for the scattering lengths are

$$
\alpha_{1 / 2} \approx-2 \alpha_{3 / 2} \approx 3
$$

in lattice units. The terms proportional to $t^{-1 / 2}$ for $t_{K}=14$ become 0.3 and -0.1 for $I=1 / 2$ and $I=3 / 2$ respectively. These terms are to be compared to 
the unit terms within square brackets in equations 5.3.2. Even though the $t^{-1 / 2}$ terms may not be small compared to one, the estimated variation of the quantity within square brackets over the interval $[12,16]$ is at most $3 \%$ for the numerical estimates above. This weak time dependence combined with the limited range of $t_{K}$ will make it difficult to separate out the $t^{-1 / 2}$ dependence.

Fits to the general model fursction

$$
f(t ; \vec{a})=a_{1}\left[1+a_{2} t^{-1 / 2}+a_{3} t^{-3 / 2}\right]+a_{4} e^{a_{5} t}
$$

were attempted for the $\mathrm{TI}$ ratios for $G_{1 / 2}, G_{3 / 2}$ and to the ratio $G_{1 / 2} / G_{3 / 2}$. Parameters were allowed to vary freely or optionally they could be frozen at particular values. Thus for the TI ratio of $G_{3 / 2} c_{4}$ and $c_{5}$ were frozen at zero since the exponential term is not expected to contribute to the $3 / 2$ TI ratio. The time-slice $t=11$ was not used for fits to prevent the pion and kaon from sitting on the same time-slice. The upper range of time-slices for fits was limited by the lattice boundary. The fits were then restricted to the range $[12,16]$.

Since Eq. (5.3.4) is not known to be the exact functional form of the TI ratio and only a limited number of points with relatively large uncertainties were fit, we conservatively limited fits to those with only two or three free parameters. For example, one could neglect the $t^{-3 / 2}$ term entirely by keeping $a_{3}$ fixed at zero. For fits to the 'TI ratio of $G_{1 / 2}$ or to the ratio of $G_{1 / 2}$ to $G_{3 / 2}$ where there is a possible exponential term one can freeze $c_{5}$ at the value $\delta m$ found from the fits disussed in Sect. 5.2.

The result of all these attempted fits is that including various time dependent terms does decrease the $\chi^{2}$ per degree of freedom of the fits. However, the estimated uncertainties in the fitted parameters generally become so large that there is little information in the fits. 
Two examples of fits to the ratio $G_{1 / 2} / G_{3 / 2}$ using Eq. (5.3.4) as the model function are shown in the following table and the accompanying figures:

\begin{tabular}{|c|c|c|c|c|c|c|}
\hline \multirow{2}{*}{$\begin{array}{l}\text { Figure } \\
\text { number }\end{array}$} & \multicolumn{5}{|c|}{ fit parameters } & \multirow{2}{*}{$\frac{x^{2}}{n}$} \\
\hline & $a_{1}$ & $a_{2}$ & $a_{3}$ & $a_{4}$ & $a_{5}$ & \\
\hline 5.3 .4 & $22(7)$ & $1(15)$ & $0^{*}$ & $-35(156)$ & $-.038^{*}$ & $\frac{.61}{2}$ \\
\hline 5.3.5 & $6(24)$ & $-4(7)$ & $0^{*}$ & $5(7)$ & $+.038^{*}$ & $\frac{.63}{2}$ \\
\hline
\end{tabular}

The values in the table followed by an asterisk are held fixed during the fit. Both fits neglect the $a_{3}$ term. The first fit takes $a_{5}$ equal to the value of $\delta m$ found from propagator fits. The second fit fixes $a_{5}$ to $-\delta m$. This value is within the uncertainty range reported for $\delta m$.

These fits are to be compared with the fit of the same quantity to the constant function $f\left(t ; a_{1}\right)=a_{1}$ shown in Sect. 1.19. The $\chi^{2}$ fit to the constant function yields $a_{1}=5.1(18)$ with a $\chi^{2}$ per degree of freedom of $7.4 / 4$.

Fits containing statistically significant information will require using time-slices spanning a range in physical time units large enough that the time variation of the functions is clearly resolved relative to the estimated uncertainties. Care must be taken to reduce the estimated uncertainties by using a large set of configurations and by trying to reduce correlations among configurations.

Determining the time dependent terms in Eq. (5.3.2) and thus extracting quantities such as $\mathcal{A}_{1 / 2}$ and $\mathcal{A}_{3 / 2}$ has proven extremely difficult given the lattices studied so far. The best we can do is neglect time dependent terms. Thus, in fits to the TI ratios of the functions in Eq. (5.3.1) that determine $\mathcal{A}_{1 / 2}$ and $\mathcal{A}_{3 / 2}$, the constant function is used as the model function rather than a function such as Eq. (5.3.4). The single parameter obtained from each of these fits is taken as the best estimate of corresponding amplitude. 


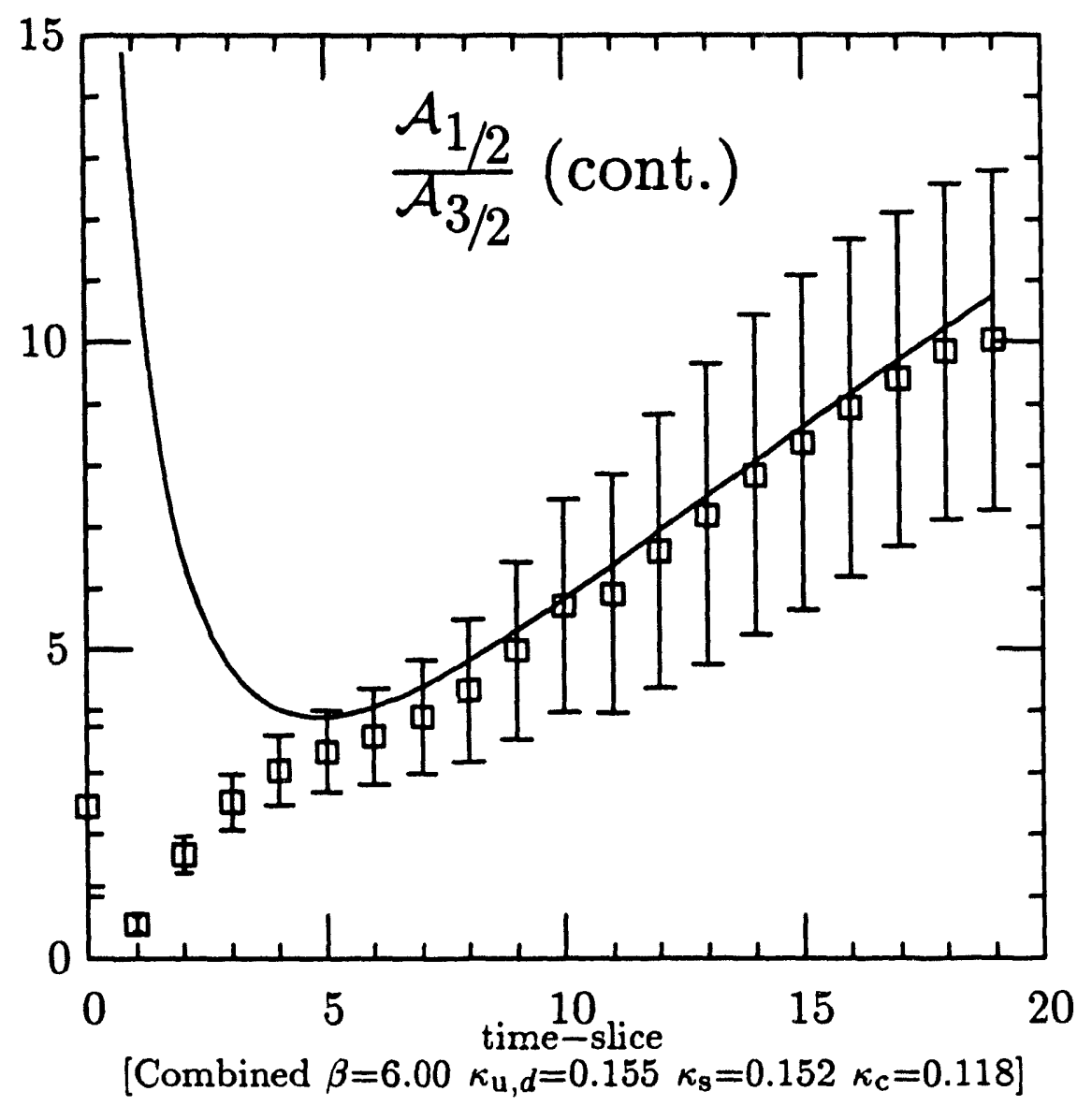

Figure 5.3.4: Fit to the model function Eq. (5.3.4) keeping the parameters $a_{3}=0$ and $a_{5}=-.038$ fixed. The value of $a_{5}$ is equal to $\delta m$ shown in Table I.5.1.

From the numerical estimates of the time dependent terms discussed previously in this section we conclude that amplitudes obtained by neglecting any time dependence can differ by $30 \%$ or more from the amplitudes that one would obtain if the time dependence could be determined. 


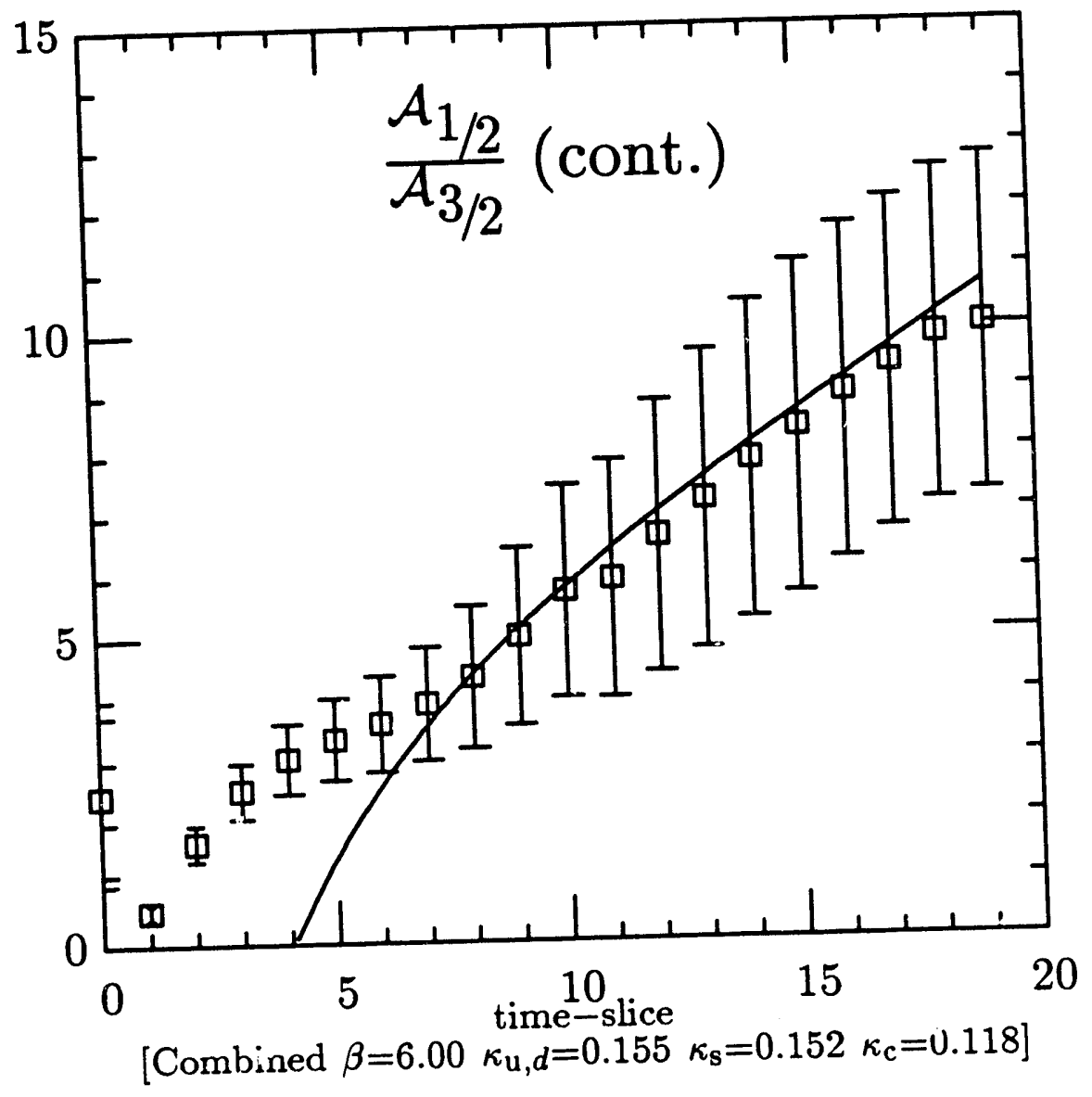

Figure 5.3.5: Fit to the model function Eq. (5.3.4) keeping the parameters $a_{3}=0$ and $a_{5}=+.038$ fixed. The value of $a_{5}$ is equal to $-\delta m$ a value within the uncertainty range of $\delta m$.

\section{4. $\mathcal{A}_{1 / 2}$ and $\mathcal{A}_{3 / 2}$ Amplitudes}

The amplitudes $\mathcal{A}_{1 / 2}$ and $\mathcal{A}_{3 / 2}$ for $D \rightarrow K \pi$ decays were determined on the lattice by taking the naire plateau value of the corresponding TI ratio as discussed in the previous section. The resulting fits and sample graphs of the time behavior of the $\mathrm{TI}$ ratios are shown for the combined $\beta=6.0$ lattices in the numerical results appendix. Similar fits were performed on the $\beta=5.7$ lattice.

Figures 5.4.1 and 5.4.2 show values of $\mathcal{A}_{3 / 2}$ and $\mathcal{A}_{1 / 2}$ as a function of the pion mass for the $\beta=6.0$ and the $\beta=5.7$ lattices. The values on the abscissa of each 


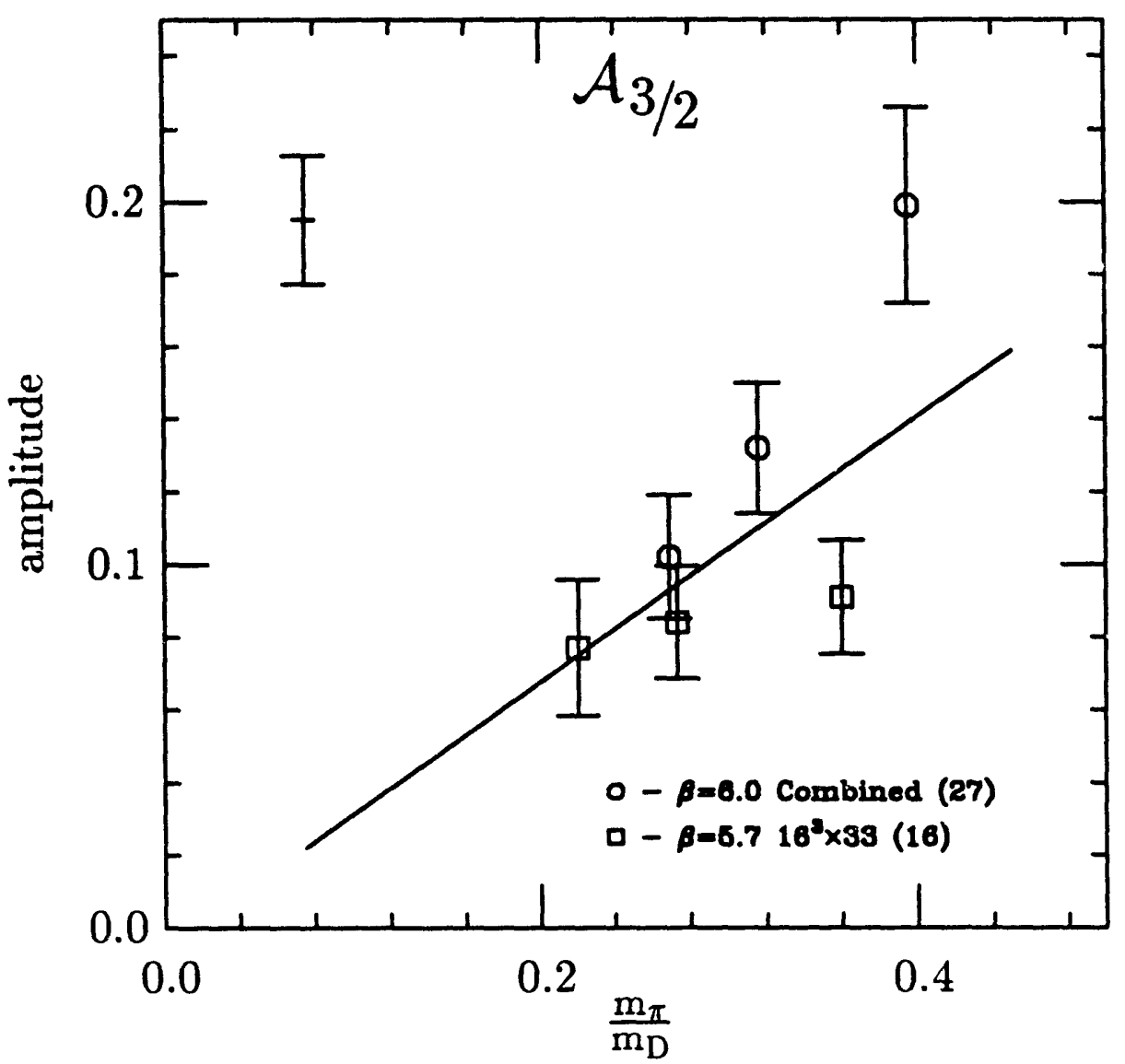

Figure 5.4.1: Graph of $\mathcal{A}_{3 / 2}$ versus $m_{\pi} / m_{D}$. The point in the upper left corner is the amplitude derived from experiment. The amplitude is scaled in units of $\frac{G_{f} V_{c s}^{*} V_{u d}}{2 \sqrt{2}}$. The line does not take into account correlations between plotted points.

figure are the ratio of the pion mass to the $D$ meson mass. Plotting the amplitudes as a function of the unitless ratio of masses reduces the systematic uncertainty in the horizontal placement of the $\beta=6.0$ points relative to the $\beta=5.7$ points due to the uncertainty in the relative values of $a^{-1}$ for the $\beta=5.7$ and $\beta=6.0$ lattices.

The amplitudes are all scaled in units of $G_{f} V_{c s}^{*} V_{u d} / \sqrt{8}$. As in Eq. (4.8.5) these amplitudes are proportional to $a^{-3}$. The absolute normalization of the amplitudes 


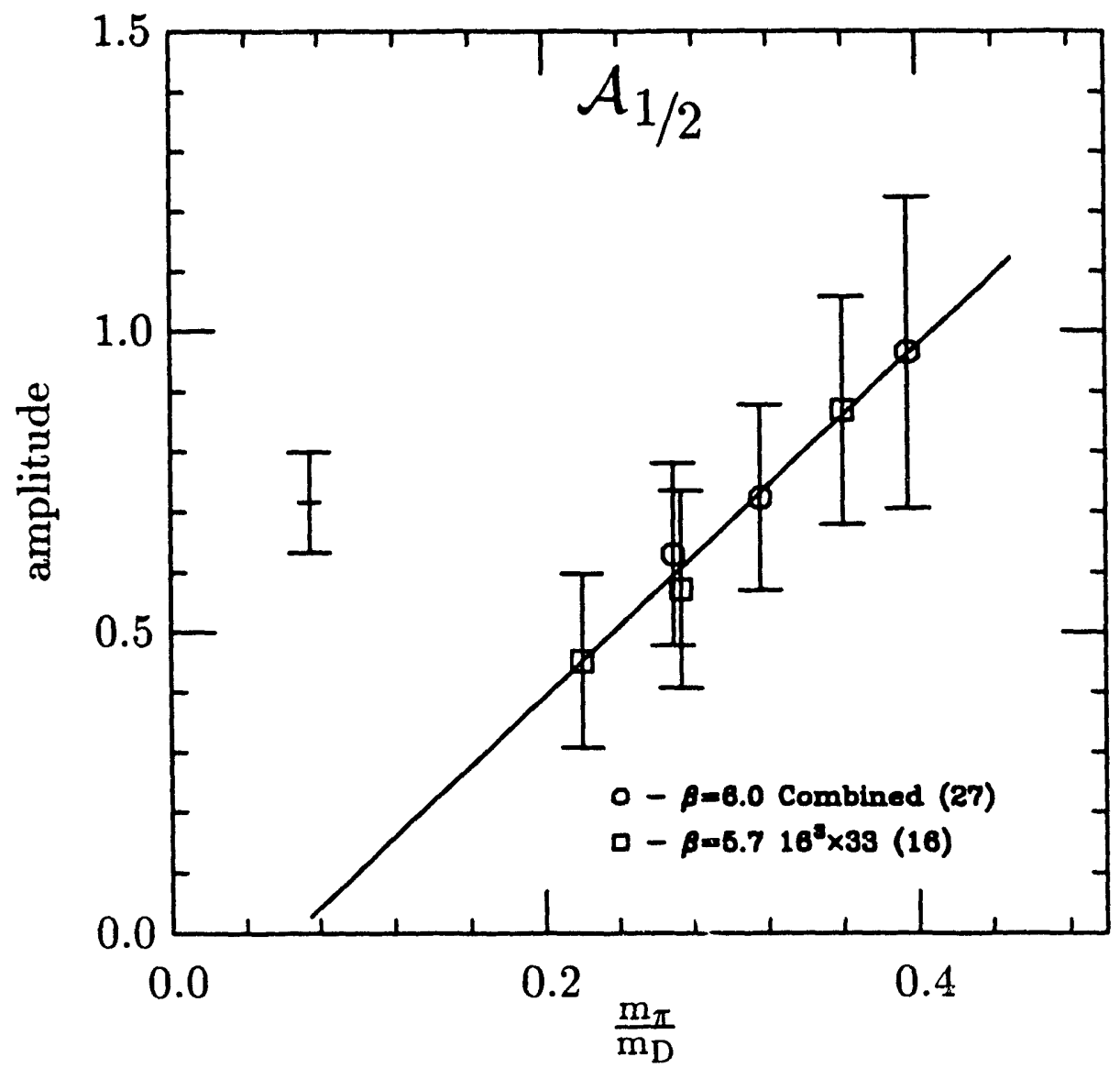

Figure 5.4.2: Graph of $\mathcal{A}_{1 / 2}$ versus $m_{\pi} / m_{D}$. The point at center left is the amplitude derived from experiment. The amplitude is scaled in units of $\frac{G_{f} V_{c s}^{*} V_{u d}}{2 \sqrt{2}}$. The line does not take into account correlations between plotted points.

are then extremely sensitive to the value of $a^{-1}$ used. The relative uncertainty between the nominal values of $a^{-1}$ for the two sets of points at different $\beta$ values can also lead to a systematic shift of one data set relative to the other set. The ratio of $a^{-1}$ at the two $\beta$ values seems satisfactory given that the two sets of data seem to overlap within the error bars in the two figures. For these graphs the nominal values of $a^{-1}$ shown in Table 5.1.1 were used. Based upon the range of $a^{-1}$ values determined from string tension measurements versus values obtained 
from hadron mass spectroscopy, we believe the systematic uncertainty in $a^{-1}$ to be about $20 \%$. Thus points with a given $\beta$ may vary by a factor of .5 to 1.7 times the values shown.

The two figures also show the isospin amplitudes derived from an analysis of the experimental $D$ decay data by the MARK III Collaboration ${ }^{14}$. The experimentally derived point is the left-most point in each figure.

The lattice vaiues are comparable in magnitude to the experimentally derived amplitudes. This gives us confidence that the matching of the lattice values to the continuum has been calculated correctly.

The fits shown in the figures are $\chi^{2}$ minimization fits to a straight line. The fits were obtained by combining the $\beta=6.0$ results and the the $\beta=5.7$ results. The plotted error bars were taken as the uncorrelated estimate of the un:ertainty in the fitted points. Points calculated on the same lattice are highly correlated. Therefore, these lines are offered as a "guide to the eye" and may only suggest a possible trend in the information shown. A serious extrapolation to the physical pion mass must await a more sophisticated analysis capable of properly accounting for correlations among the computed points.

\subsection{Mass Dependence of Amplitudes}

Note that the trend in the $\dot{\mathcal{A}}_{1 / 2}$ and $\mathcal{A}_{3 / 2}$ figures suggests a dependence on the pion mass. Both amplitudes tend to decrease as the simulation pion mass approaches the chiral limit. This mass dependence was first stressed by the ELC group $^{39}$. 


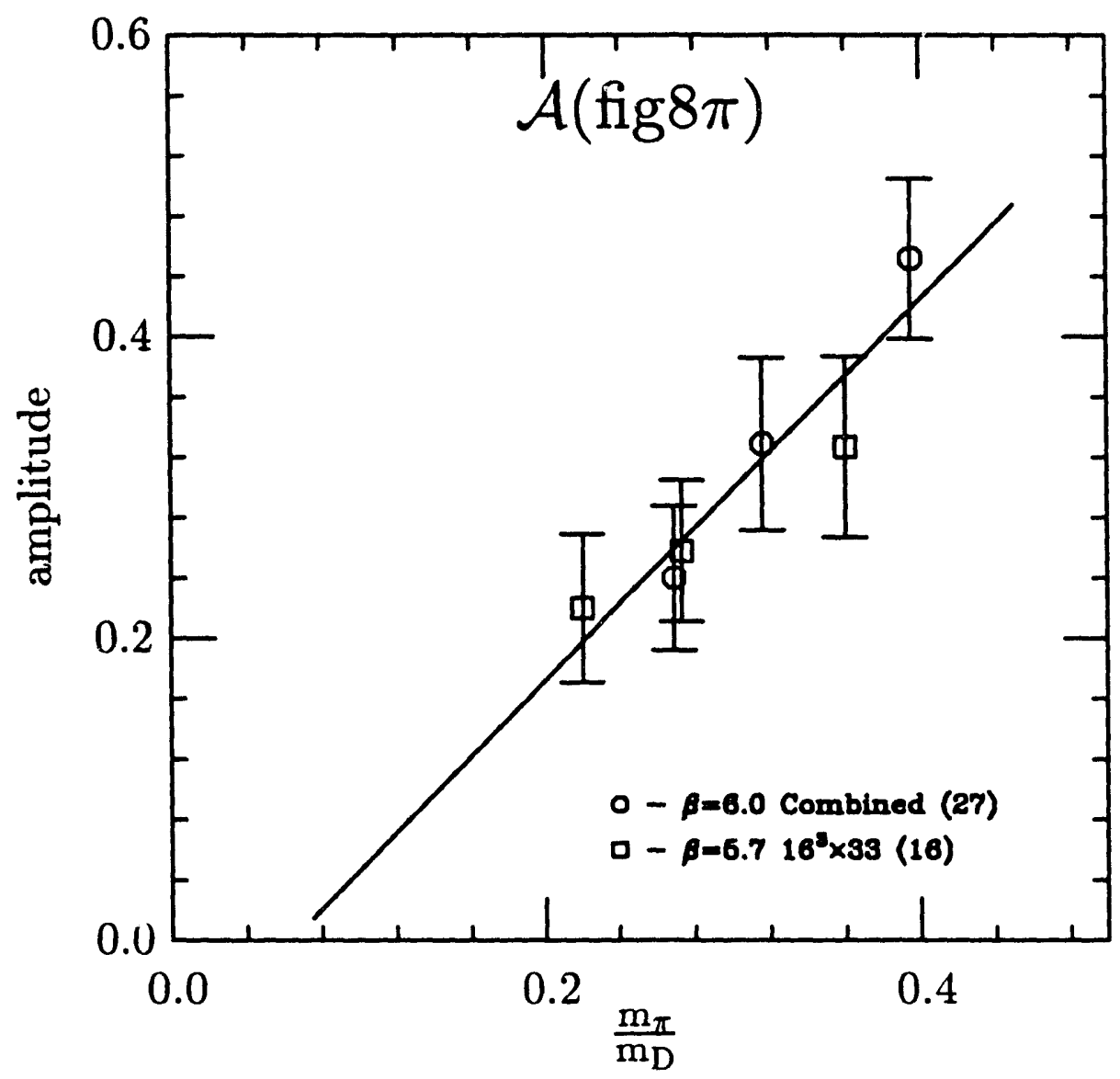

Figure 5.5.1: Graph of $\mathcal{A}($ fig $8 \pi)$ versus $m_{\pi / m_{D}}$. The line does not take into account correlations between plotted points.

We examine the pion mass dependence of the correlation functions fig $8 \pi$, fig $8 \mathrm{~K}$, and fig8an in Figures 5.5.1, 5.5.2, and 5.5.3 respectively. The abscissa and ordinate of each graph is scaled to the same units as the $\mathcal{A}_{1 / 2}$ and $\mathcal{A}_{3 / 2}$ amplitudes. The figure eight amplitudes have the same $a^{-3}$ dependence as do the isospin amplitudes.

Again, the lines drawn through the measured points are a $\chi^{2}$ minimization assuming that the displayed uncertainties are uncorrelated. These lines are offered as an illustration of gross trends in the points shown. 


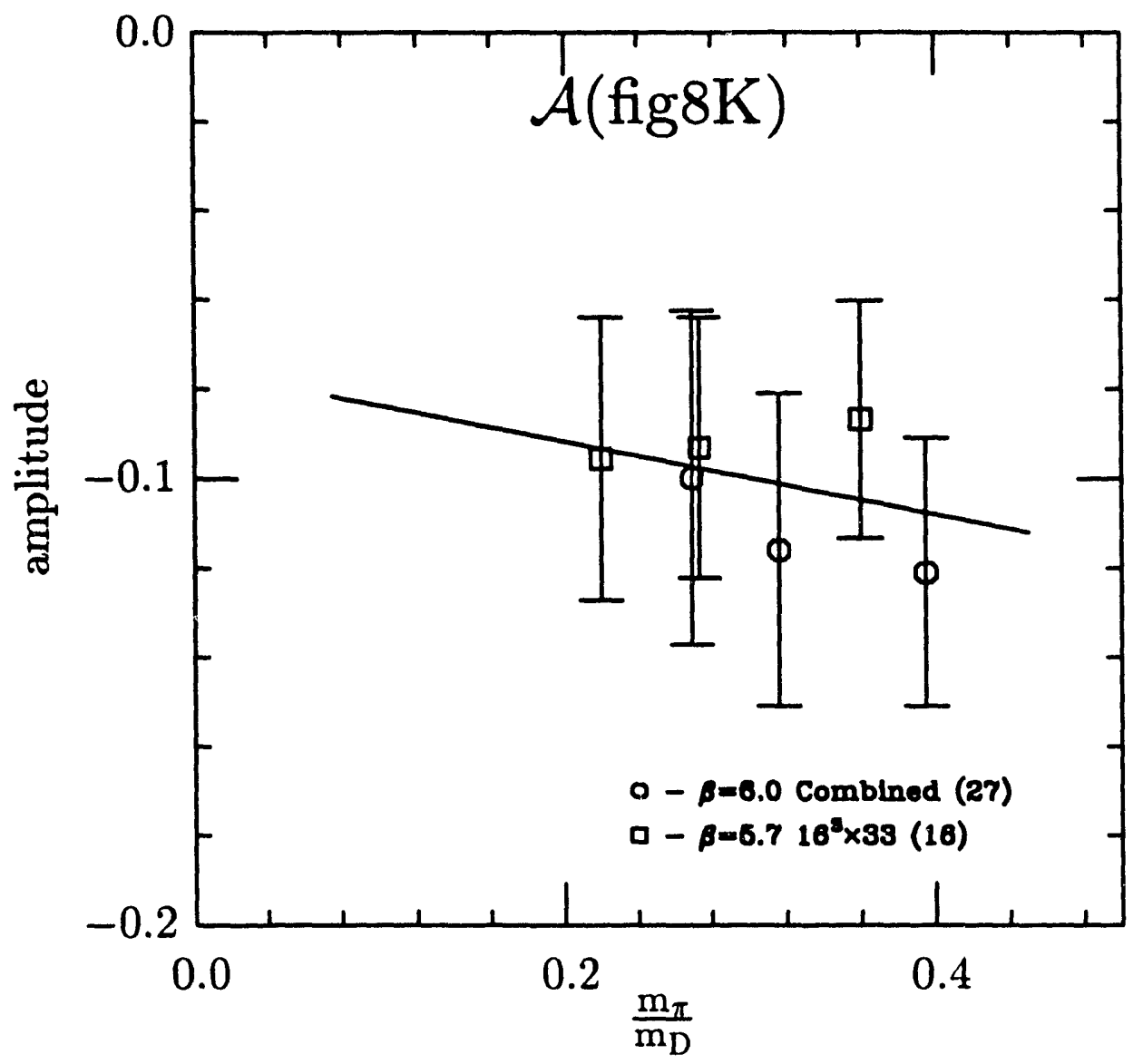

Figure 5.5.2: Graph of $\mathcal{A}($ fig $8 \mathrm{~K})$ versus $m_{\pi} / m_{D}$. The line does not take into account correlations between plotted points.

Note that the pion mass dependence in the fig $8 \pi$ and fig8an amplitudes is apparently much more drastic than for the fig $8 \mathrm{~K}$ amplitude. The lines through the measured points for fig $8 \pi$ and fig8an seem to suggest a direct proportionality of these amplitudes to the pion mass. The amplitude for fig8K seems to approach a non-zero value at the physical pion mass.

We attempt to understand the momentum dependence of these amplitudes by studying continuum models. The lattice amplitudes are calculated "at rest" (see Sect. 4.4). The $\pi, K$, and $D$ particles then have four-momenta

$$
p_{\pi}=\left(m_{\pi}, \overrightarrow{0}\right)
$$




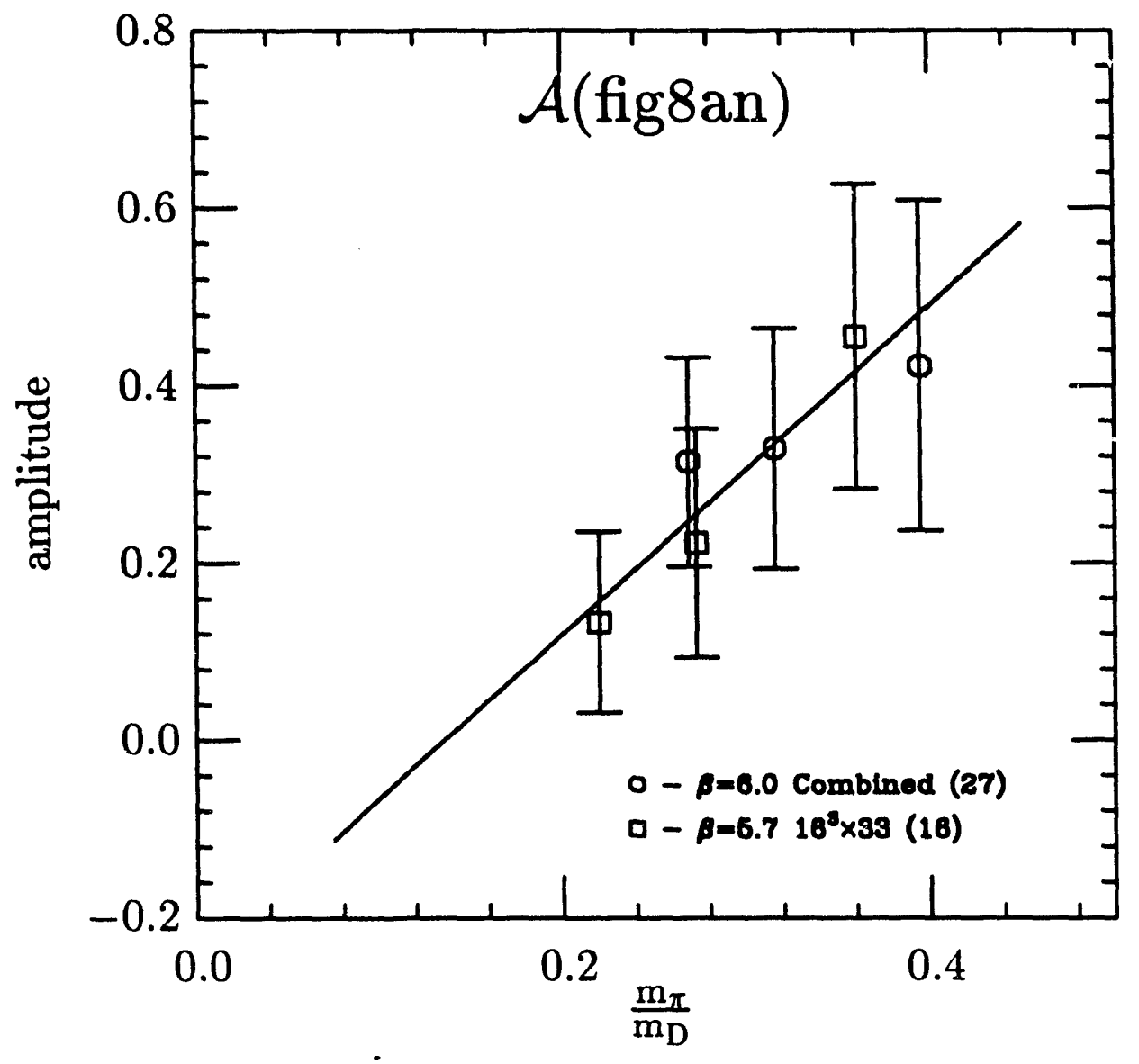

Figure 5.5.3: Graph of $\mathcal{A}\left(\right.$ fig8an) versus $m_{\pi} / m_{D}$. The line does not take into account correlations between plotted points.

$$
\begin{aligned}
& p_{K}=\left(m_{K}, \overrightarrow{0}\right) \\
& p_{D}=\left(m_{D}, \overrightarrow{0}\right)
\end{aligned}
$$

Therefore, the lattice amplitudes are calculated at a point in momenta space where total three-momentum is conserved but energy is not. Continuum models should then be studied subject to the same "at rest" kinematical constraint. We then compute the "at rest" $D \rightarrow K \pi$ amplitudes using the vacuum saturation approximation $^{48}$.

Start with $\left\langle K \pi\left|\mathcal{H}_{w}^{e f f}\right| D\right\rangle$ where $\mathcal{H}_{w}^{\text {eff }}$ is defined in Eq. (3.2.23). The vacuum state is inserțed into the matrix element in all possible ways. Among the resulting 
arıplitudes take, as an example, the fig $8 \pi$ amplitudes:

$$
\begin{aligned}
& \mathcal{A}^{V S}\left(\text { fig8 } 8 \pi_{1}\right)=\left\langle\pi\left|-\overline{\mathrm{u}}^{a} A^{\mu} \mathrm{d}^{a}\right| 0\right\rangle\left\langle K\left|\overline{\mathrm{s}}^{b} V_{\mu} \mathrm{c}^{b}\right| D\right\rangle \\
& \mathcal{A}^{V S}\left(\text { fig } 8 \pi_{2}\right)=\left\langle\pi\left|-\overline{\mathrm{u}}^{a} A^{\mu} \mathrm{d}^{b}\right| 0\right\rangle\left\langle K\left|\overline{\mathrm{s}}^{b} V_{\mu} \mathrm{c}^{a}\right| D\right\rangle
\end{aligned}
$$

where the other possible insertions of the vacuum state are recognized as the $\mathcal{A}$ (fig8K) and $\mathcal{A}($ fig8an $)$ amplitudes. The indices $a$ and $b$ are color indices. The Fierz relation Eq. (4.5.37) was used in the derivation of Eq. (5.5.2b) in order to rearrange the fermion fields in $\mathcal{O}_{2}$.

The matrix element of the axial current $\overline{\mathrm{u}}^{a} A^{\mu} \mathrm{d}^{b}$ is parameterized in terms of the pion decay constant $f_{\pi}$

$$
\left\langle\pi\left|\overline{\mathrm{u}}^{a} A^{\mu} \mathrm{d}^{b}\right| 0\right\rangle=\frac{\delta_{a b}}{N} f_{\pi} p_{\pi}^{\mu} .
$$

The three-point function involving the vector current $\overline{\mathrm{s}}^{b} V_{\mu} \mathrm{c}^{a}$ can be parameterized in the helicity basis ${ }^{7}$

$$
\begin{aligned}
\left\langle K\left|\overline{\mathrm{s}}^{b} V_{\mu} \mathrm{c}^{a}\right| D\right\rangle= & {\left[\left(p_{D}+p_{K}\right)_{\mu}-\frac{m_{D}^{2}-m_{K}^{2}}{q^{2}} q_{\mu}\right] f_{b a}^{(+)}\left(q^{2}\right)+} \\
& \frac{m_{D}^{2}-m_{K}^{2}}{q^{2}} q_{\mu} f_{b a}^{(0)}\left(q^{2}\right)
\end{aligned}
$$

where

$$
\begin{aligned}
f_{b a}^{(+)}\left(q^{2}\right) & =\frac{\delta_{b a}}{N} f^{(+)}\left(q^{2}\right) \\
f_{b a}^{(0)}\left(q^{2}\right) & =\frac{\delta_{b a}}{N} f^{(0)}\left(q^{2}\right)
\end{aligned}
$$

and the form factors satisfy $f^{(+)}(0)=f^{(0)}(0)$. The momentum transfer $q_{\mu}$ is $q_{\mu}=\left(p_{D}-p_{K}\right)_{\mu}$. 
Combining Eq. (5.5.3) and Eq. (5.5.4) with the "at rest" kinematic constraint yields for fig $8 \pi$

$$
\begin{aligned}
& \mathcal{A}\left(\text { fig8 } 8 \pi_{1}\right)=f_{\pi} m_{\pi}\left(m_{D}+m_{K}\right) f_{D \rightarrow K}^{(0)}\left(q^{2}\right) \\
& \mathcal{A}\left(\text { fig8 } 8 \pi_{2}\right)=\frac{1}{N} f_{\pi} m_{\pi}\left(m_{D}+m_{K}\right) f_{D \rightarrow K}^{(0)}\left(q^{2}\right)
\end{aligned}
$$

where $q^{2}=\left(m_{D}-m_{K}\right)^{2}$. Compare these expressions to the expressions derived from the same parameterizations but now with the on shell kinematical relation $q_{\mu}=\left(p_{D}-p_{K}\right)_{\mu}=p_{\pi_{\mu}}$

$$
\begin{aligned}
& \mathcal{A}\left(\text { fig8 } \pi_{1}\right)=f_{\pi}\left(m_{D}^{2}-m_{K}^{2}\right) f_{D \rightarrow K}^{(0)}\left(q^{2}\right) \\
& \mathcal{A}\left(\text { fig8 } 8 \pi_{2}\right)=\frac{1}{N} f_{\pi}\left(m_{D}^{2}-m_{K}^{2}\right) f_{D \rightarrow K}^{(0)}\left(q^{2}\right)
\end{aligned}
$$

where $q^{2}=m_{\pi}^{2}$.

The form factor $f^{(0)}\left(q^{2}\right)$ appearing in these equations is evaluated a $a^{\dagger}$ a momentum transfer that depends on which kinematical condition is selected. This form factor as a function of $q^{2}$ has previously been measured on the lattice ${ }^{49}$.

The vacuum saturation results predict a direct proportionality between the fig $8 \pi$ amplitudes and $m_{\pi}$ for the "at rest" condition. Figure 5.5.4 shows $\mathcal{A}($ fig $8 \pi)$ as determined on the lattice but now divided by the mass behavior expected from vacuum saturation and the "at rest" condition. The line drawn through the points now seems to suggest that an extrapolation to $m_{\pi}$ would give a nonzero result. The vacuum saturation calculation only accounts for the factorizable portion of the amplitude. Since the lattice results also include non-factorizable contributions, the $m_{\pi}$ mass dependence of the vacuum saturation results should only be considered an approximation to the behavior of the lattice amplitudes. 


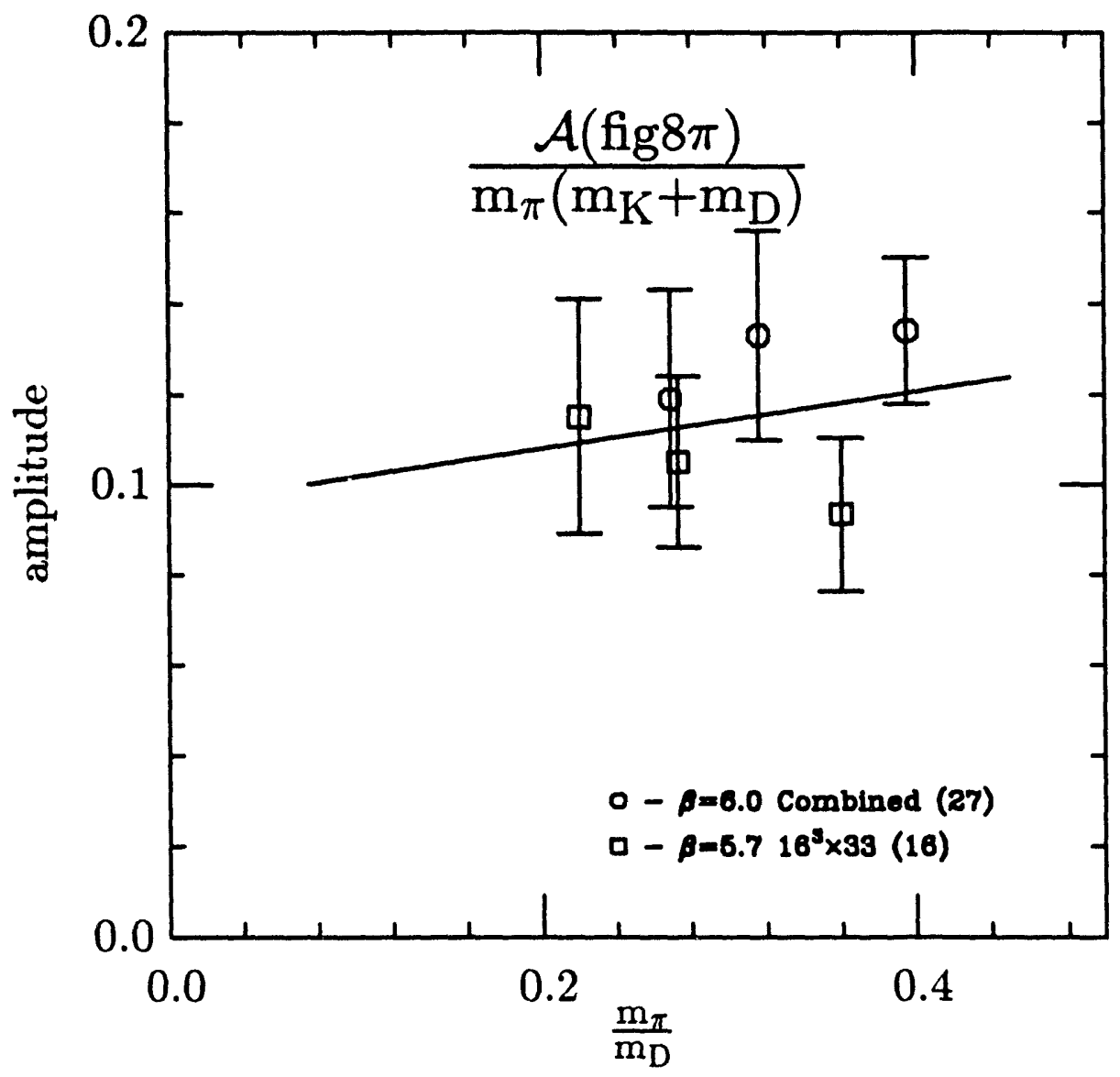

Figure 5.5.4: Graph of $\mathcal{A}($ fig $8 \pi)$ divided by the mass dependence expected for "at rest" kinematics in the continuum vacuum saturation approximation versus $m_{\pi} / m_{D}$. The line does not take into account correlations between points.

The "at rest" condition for fig $8 \mathrm{~K}$ yields in the vacuum saturation approximation

$$
\begin{aligned}
& \mathcal{A}\left(\text { fig8K }_{1}\right)=\frac{1}{N} f_{K} m_{K}\left(m_{D}+m_{\pi}\right) f_{D \rightarrow \pi}^{(0)}\left(q^{2}\right) \\
& \mathcal{A}\left(\text { fig8K }_{2}\right)=f_{K} m_{K}\left(m_{D}+m_{\pi}\right) f_{D \rightarrow \pi}^{(0)}\left(q^{2}\right)
\end{aligned}
$$

where $q^{2}=\left(m_{D}-m_{\pi}\right)^{2}$. The fig8K amplitude is then expected to be much less sensitive to a decrease in the pion mass. Figure 5.5.2 is apparently consistent with this observation. 
Finally, for fig8an the "at rest" amplitudes are

$$
\begin{aligned}
& \mathcal{A}\left(\text { fig8an }_{1}\right)=\frac{1}{N} f_{D} m_{D}\left(m_{K}-m_{\pi}\right) f_{K \rightarrow \pi}^{(0)}\left(q^{2}\right) \\
& \mathcal{A}\left(\text { fig8an }_{2}\right)=f_{D} m_{D}\left(m_{K}-m_{\pi}\right) f_{K \rightarrow \pi}^{(0)}\left(q^{2}\right)
\end{aligned}
$$

where $q^{2}=\left(m_{K}+m_{\pi}\right)^{2}$. Crossing symmetry has been used to write $\left\langle K \pi\left|\overline{\mathrm{s}} V_{\mu} \mathrm{d}\right| 0\right\rangle$ in terms of the matrix element $\left\langle\pi\left|\bar{s} V_{\mu} \mathrm{d}\right| \pi\right\rangle$.

The factorizable contributions to fig8an are proportional to $m_{K}-m_{\pi}$ and therefore vanish in the $S U(3)_{f}$ limit. The mass dependence shown in Fig. 5.5.3 seems to depart from this predicted behavior. The right-most two points of Fig. 5.5.3 are calculated in the $S U(3)$ limit. Instead of vanishing at these two points, the amplitude is apparently large. This may be an indication that the non-factorizable contributions neglected in Eq. (5.5.9) are significant for fig8an.

\subsection{Ratios}

Ratios are studied on the lattice for three reasons. First, remember that the lattice $D$ decay amplitudes regain their appropriate dimensional units by multiplication by $a^{-3}$. Since the inverse lattice spacing $a^{-1}$ is considered to have a large systematic uncertainty, the amplitudes have a large systematic uncertainty. On the other hard, ratios of amplitudes, are dimensionless and thus less sensitive to scale uncertainties. Second, lattice quantities are all highly correlated. One hopes that the correlated uncertainties in the numerator and the denominator of a ratio can be combined to yield a ratio with a significance perhaps greater than the significance to which either the numerator or denominator alone can be determined. Finally, it is easiest to make contact with continuum models through ratios. For example, in the vacuum saturation approximation taking the $S U(3)$ limit allows 


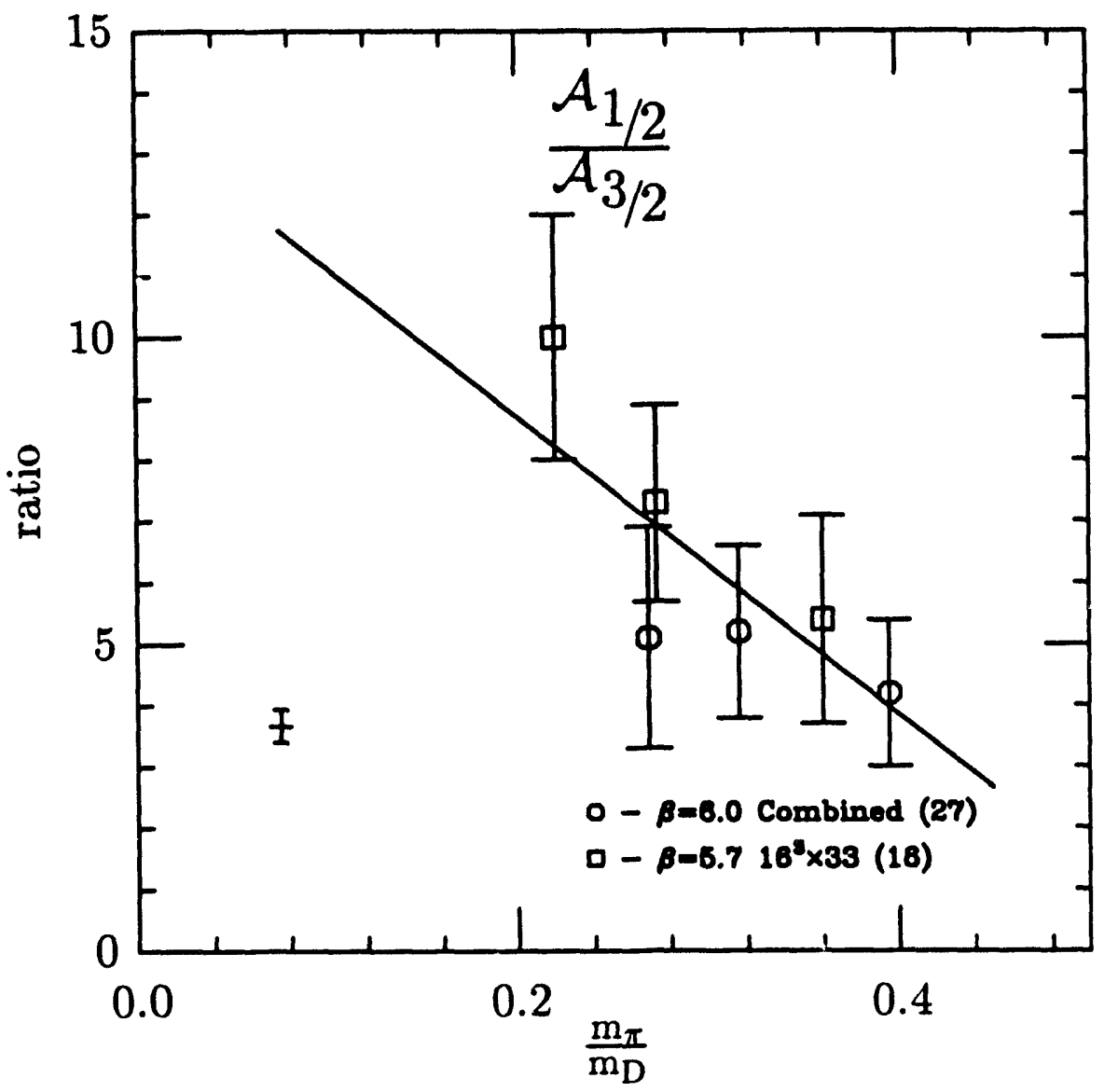

Figure 5.6.1: Graph of the ratio of isospin amplitudes $\mathcal{A}_{1 / 2} / \mathcal{A}_{3 / 2}$ versus $m_{\pi} / m_{D}$. The point at lower left is the ratio derived from experiment. The line does not take into account correlations between plotted points.

a cancellation between the numerator and denominator of the form factors and decay constants that appear as parameters in the formulas for the amplitudes.

Figure 5.6.1 shows values of the ratio of $\mathcal{A}_{1 / 2}$ to $\mathcal{A}_{3 / 2}$ amplitudes. The ratios are calculated by $\chi^{2}$ fits to a constant function. Possible time dependencies in the TI ratio are simply neglected since the time depeindent terms cannot currently be resolved. Some of the details for these fits are found in section I.19. Note from table I.20.1 that the $\chi^{2}$ values for fits to the constant model function are large. 


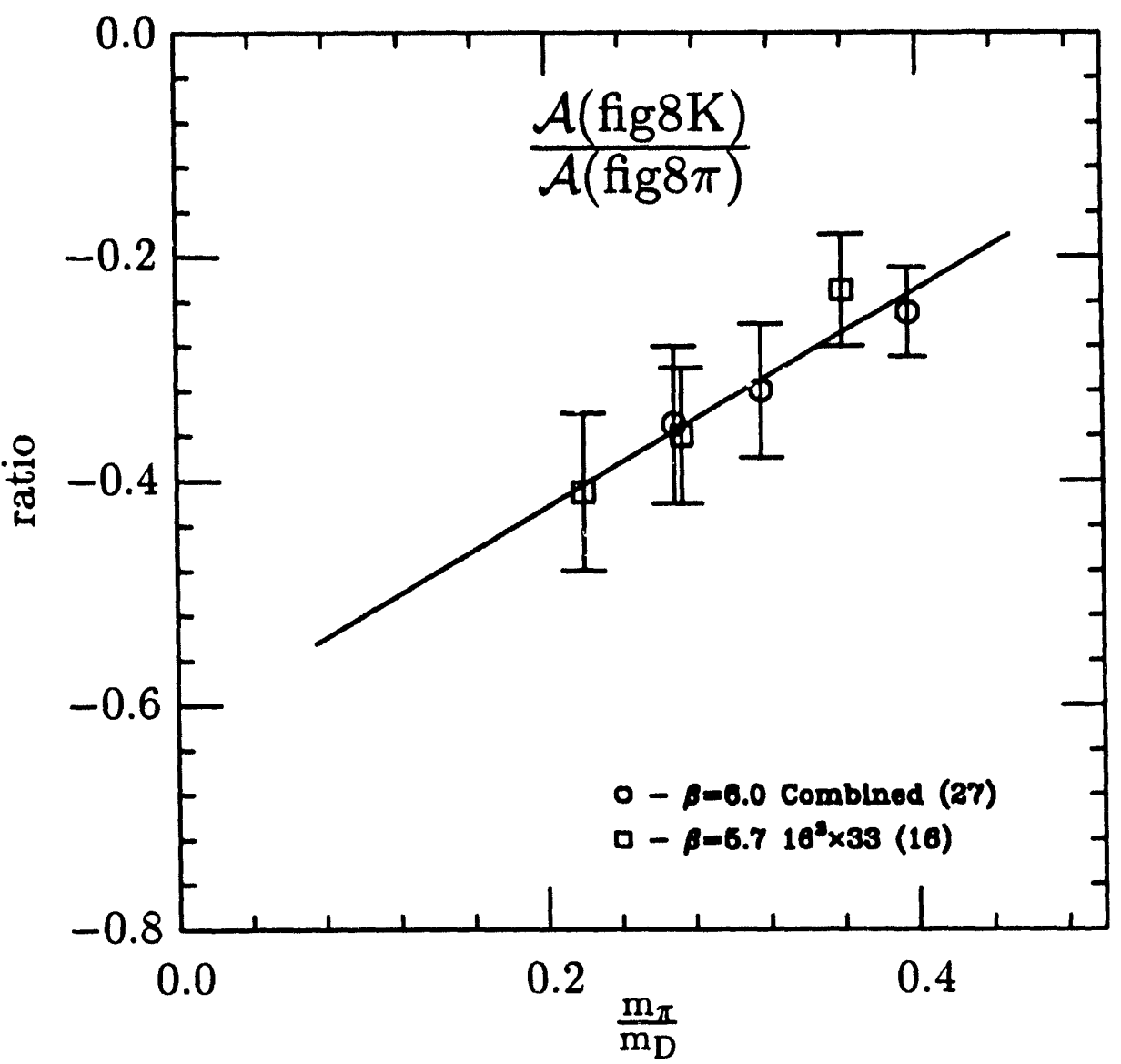

Figure 5.6.2: The ratio $\mathcal{A}(\mathrm{fig} 8 \mathrm{~K}) / \mathcal{A}($ fig $8 \pi)$ versus $m_{\pi} / m_{D}$. The negative value of this ratio shows destructive interference between the spectator and the color suppressed spectator process. The line does not take into account correlations between plotted points.

The sample plot of the TI ratio and the accompanying fit in figure I.19.1 shows that the fit to the lattice results is poor.

The point at bottom left of figure 5.6.1 shows the experimentally derived isospin amplitude ratio as found by a best fit to equations 1.5. This corresponds to the value shown in Eq. (1.8). Only a qualitative comparison can be made between the lattice results and the experimentally derived value since the lattice amplitudes are taken at rest. 


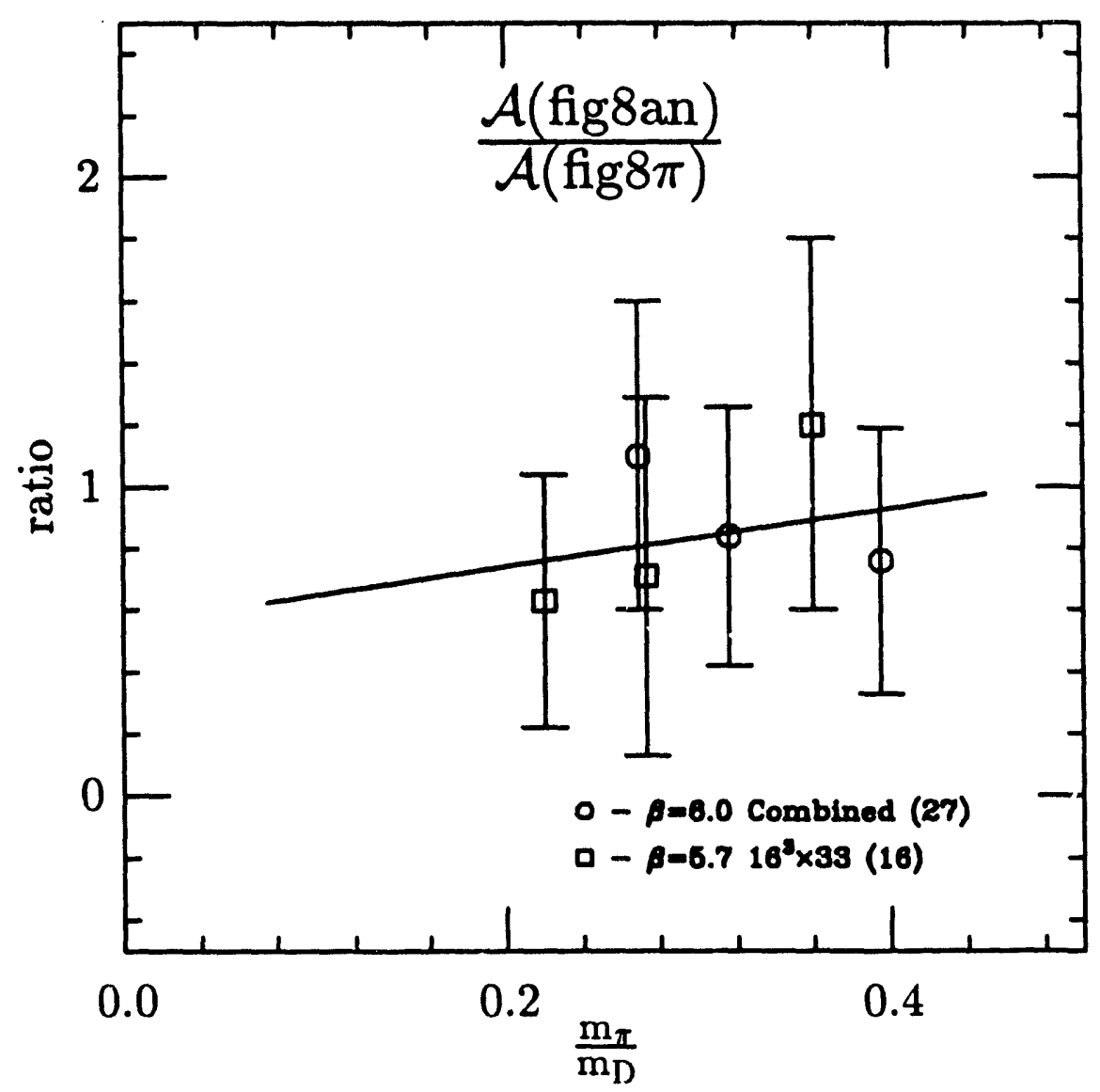

Figure 5.6.3: The ratio $\mathcal{A}($ fig8an $) / \mathcal{A}($ fig8 $8 \pi)$ versus $m_{\pi} / m_{D}$. This is the ratio of the weak annihilation process to the spectator process. The line does not take into account correlations between plotted points.

The line drawn through the points is not to be considered more than a "guide to the eye". This line does not take into account the highly correlated nature of the points being fitted. The trend indicated by this line for the combined $\beta=6.0$ and $\beta=5.7$ points is for the ratio to increase with decreasing $m_{\pi}$. Note that the trend line may be biased upwards because of the left-most $\beta=5.7$ point. Note in particular that there is a much flatter trend in the $\beta=6.0$ points. 
Figure 5.6.2 shows the lattice results for the ratio of $\mathcal{A}(\mathrm{fig} 8 \mathrm{~K})$ to $\mathcal{A}($ fig8 8 ) as a function of the scaled pion mass. Again, the line through the points is not to be considered a proper extrapolation to the experimental pion mass.

Recall that the analogous quantity to this ratio in continuum models is the ratio of the color suppressed spectator amplitude to the spectator amplitude. Note that the lattice result is negative in sign. The two lattice amplitudes then interfere destructively.

The trend line in the figure indicates that the ratio has a mass dependence. This mass dependence may be expected based on the VS amplitudes at rest (equations 5.5.6 and 5.5.8).

Figure 5.6.3 shows the ratio of the weak annihilation amplitude to the spectator amplitude calculated on the lattice. The central values of each point show fig8an to be a significant fraction of fig $8 \pi$. However, the uncertainties preclude any definite statement. Moreover, sect. I.11 indicates that the formal $\chi^{2}$ fits are poor. The large $\chi^{2}$ value for the sample fit demonstrated in Fig. I.11.1 is most likely due to the unknown contribution of time dependent terms.

Since the at rest kinematical constraint makes it difficult to make direct comparisons between the lattice and experiment, we propose studying continuum models subject to the at rest constraint. The lattice may then serve as a check of proposed models.

Figure 5.6.4 again shows lattice results for the ratio of $1 / 2$ to $3 / 2$ isospin amplitudes. However, flavor $S U(3)$ symmetry has been implemented by setting the up down and strange quark masses equal to facilitate comparisons to the vacuum saturation approximation. Again, the line through the data points is meant to show the overall trend of the points. 


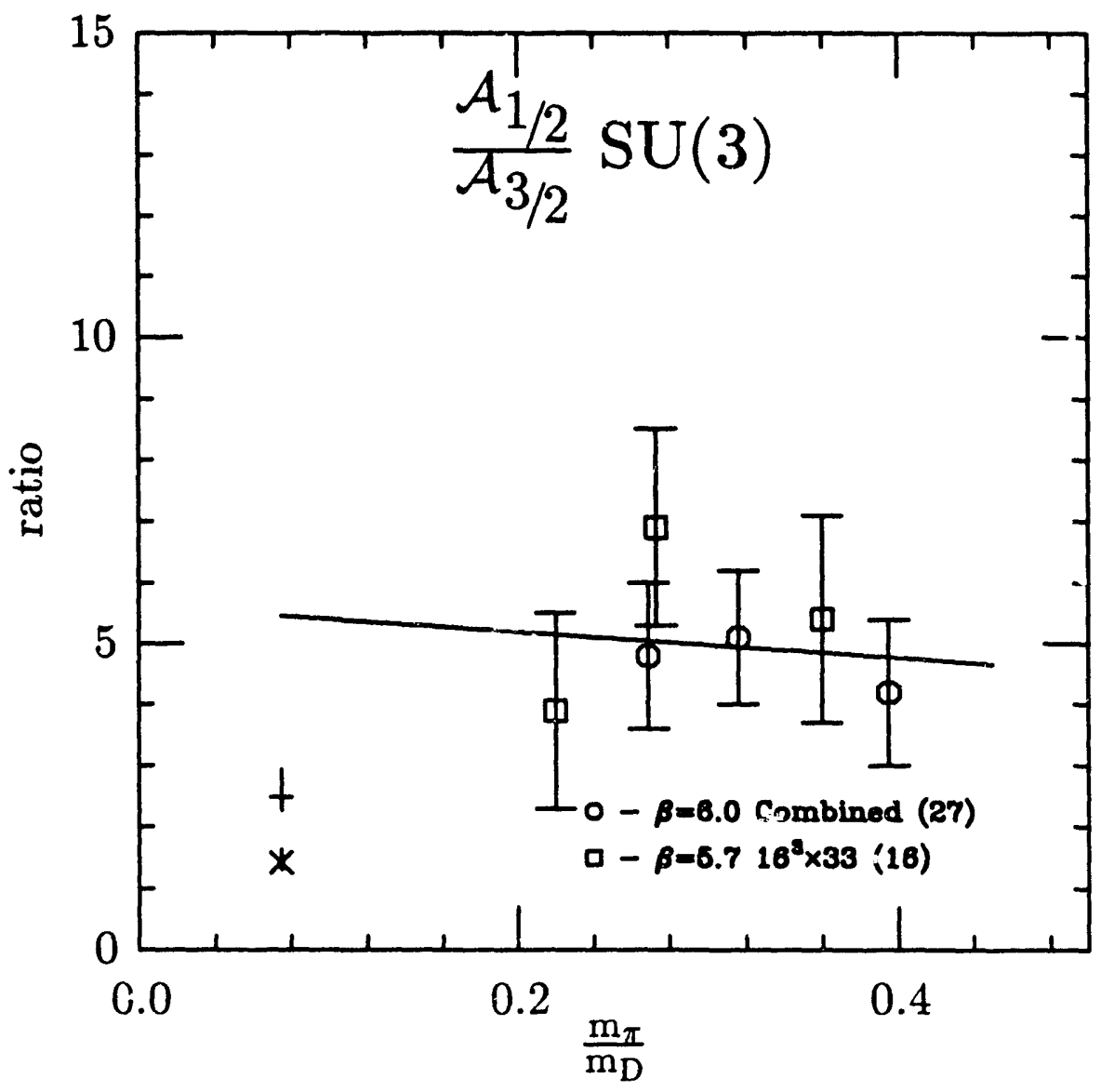

Figure 5.6.4: Graph of the ratio of isospin amplitudes $\mathcal{A}_{1 / 2} / \mathcal{A}_{3 / 2}$ versus $m_{\pi / m_{D}}$ in the flavor $S U(3)$ limit. The two points in the lower left are continuum model vacuum saturation calculations in the flavor $S U(3)$ limit. The point labeled + is the large $N$ estimate. The point labeled $\times$ retains the $1 / \mathrm{N}$ terms. The line does not take into account correlations between plotted points.

The two left-most points in the figure are vacuum saturation model predictions. The point marked ' $x$ ' is the naive vacuum saturation prediction in which the order $1 / \mathrm{N}$ terms that arise from the "Fierzed" terms are retained. The point marked ' + ' has the Fierzed terms dropped. The isospin amplitude ratio in the VS approximation is

$$
\frac{\mathcal{A}_{1 / 2}}{\mathcal{A}_{3 / 2}}=\sqrt{2} \frac{\left(1-\frac{1}{2 N}\right) c_{1}-\frac{1}{2}\left(1-\frac{2}{N}\right) c_{2}}{\left(1+\frac{1}{N}\right)\left(c_{1}+c_{2}\right)}
$$




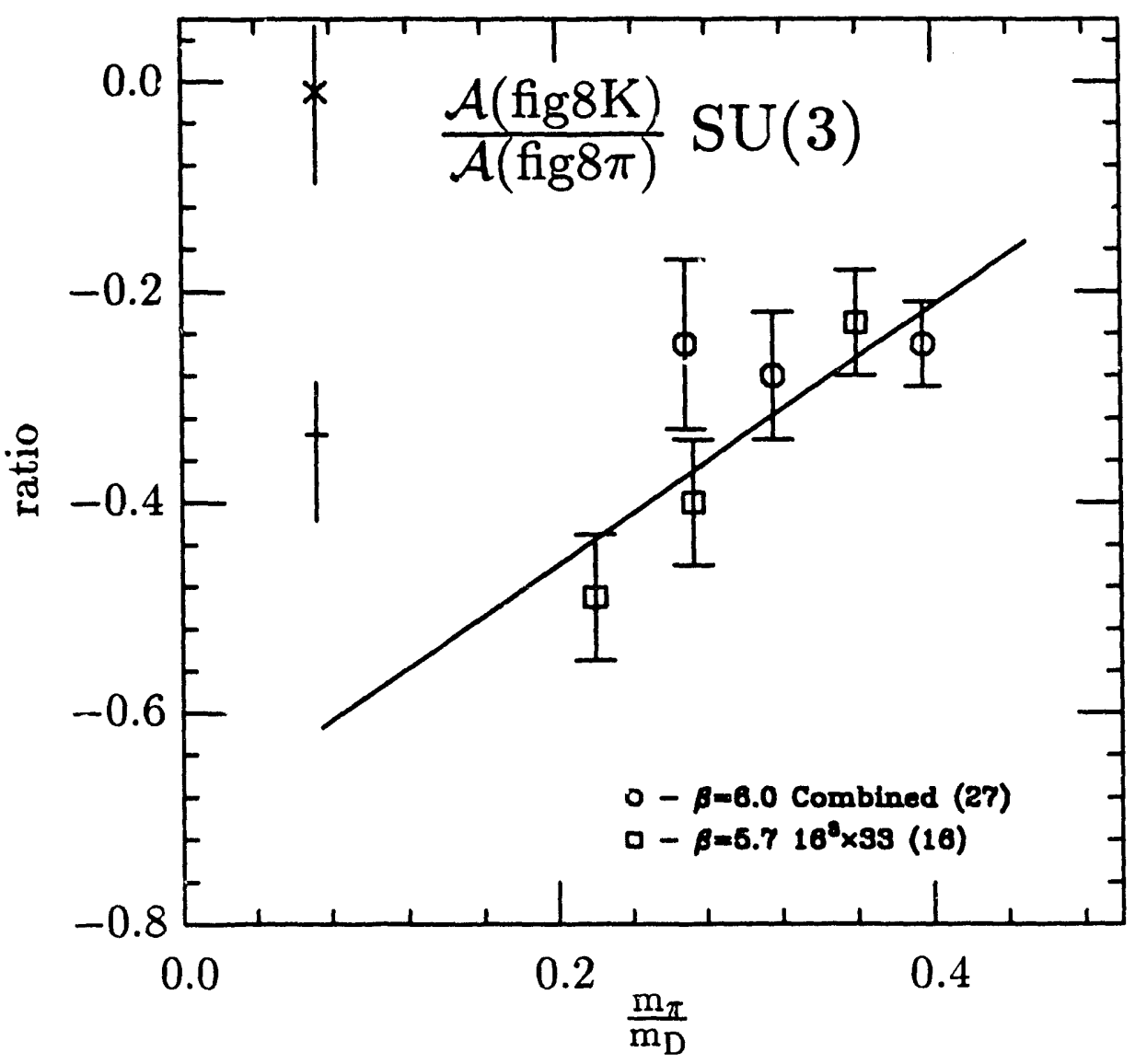

Figure 5.6.5: The ratio $\mathcal{A}($ fig $8 \mathrm{~K}) / \mathcal{A}($ fig $8 \pi)$ versus $m_{\pi} / m_{D}$ in the $S U(3)$ flavor limit. The points at middle and upper left are continuum model vacuum saturation calculations in the flavor $S U(3)$ limit. The point labeled + is the large $N$ estimate. The point labeled $\times$ retains the $1 / \mathrm{N}$ terms. The line does not take into account correlations between plotted points.

where taking $1 / \mathrm{N}=1 / 3$ yields the naive VS result and $1 / \mathrm{N}=0$ corresponds to dropping the Fierz terms. The Wilson coefficients are taken to be $c_{1}=1.19$ and $c_{2}=-0.400$ where $\mu=1.5(1) \mathrm{GeV}$ and $\Lambda \frac{(4)}{M S}=0.200\left({ }_{-80}^{+150}\right) \mathrm{GeV}$. The ranges about the VS points in the figure reflect the uncertainties in $\mu$ and $\Lambda_{\overline{M S}}$.

Recall that when $S U(3)$ flavor symmetry is imposed the VS annihilation amplitude vanishes (see Eq. (5.5.9)). However, the lattice calculation includes nonfactorizable contributions in addition to the factorizable VS contribution. 


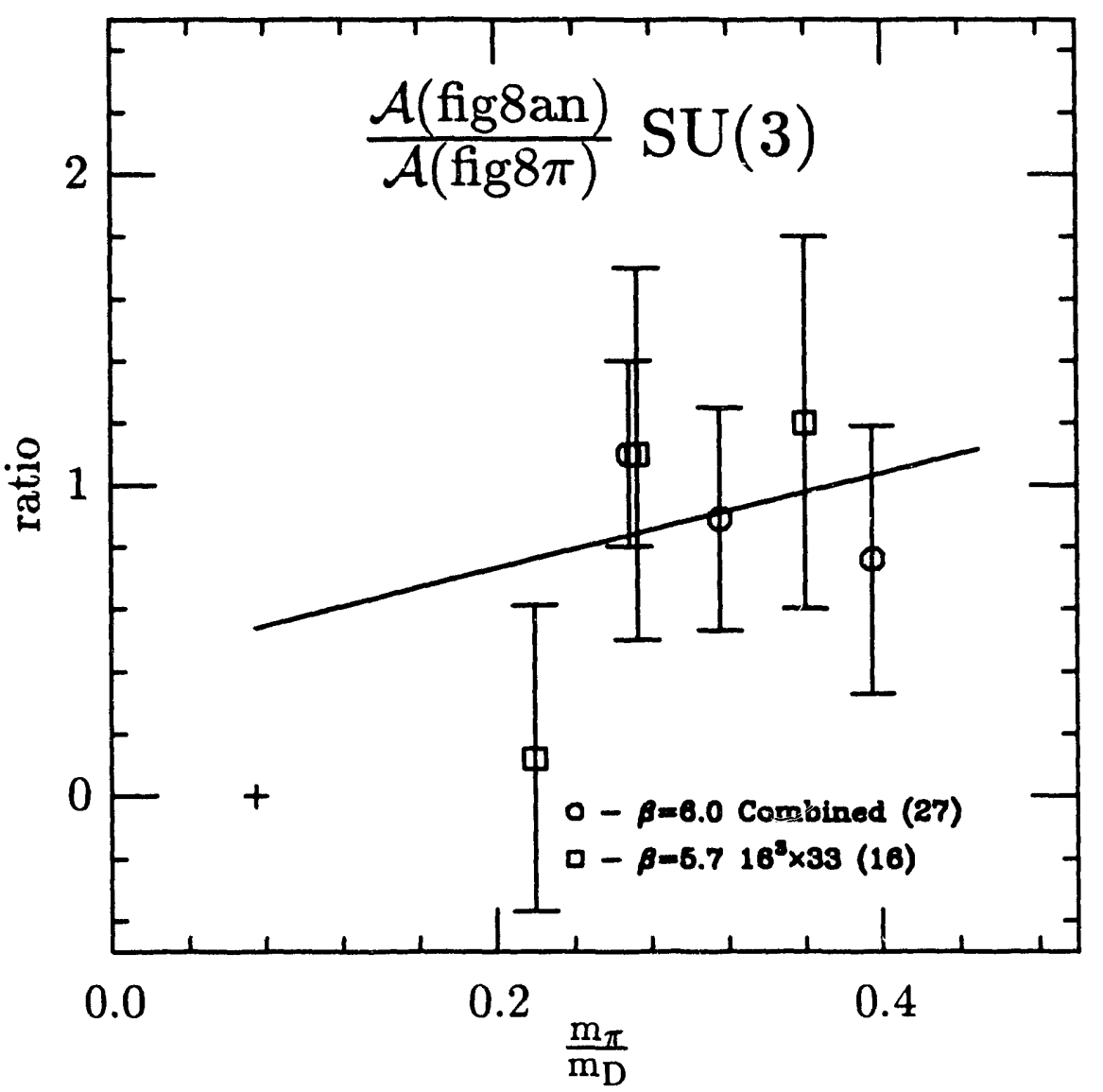

Figure 5.6.6: Graph of the ratio $\mathcal{A}$ (fig8an)/ $\mathcal{A}($ fig $8 \pi)$ versus $m_{\pi} / m_{D}$ in the $S U(3)_{f}$ limit. This is the ratio of the weak annihilation process to the spectator process. The point at zero in bottom left indicates that for a continuum vacuum saturation calculation, in the $S U(3)_{f}$ limit, the annihilation amplitude vanishes by current conservation. The line does not take into account correlations between plotted points.

Next, we examine the ratio of fig $8 \mathrm{~K}$ to fig $8 \pi$ amplitudes in the limit of $S U(3)$ flavor symmetry. The ratio is shown in Fig. 5.6.5. Again, the left-most two points are the predictions of the VS approximation. From Eq. (5.5.8) and Eq. (5.5.6) the ratio in VS approximation is given by

$$
\frac{\mathcal{A}(\text { fig } 8 \mathrm{~K})}{\mathcal{A}(\text { fig } 8 \pi)}=\frac{c_{2}+\frac{1}{N} c_{1}}{c_{1}+\frac{1}{N} c_{2}}
$$


The figure shows that keeping the Fierzed terms $(1 / \mathrm{N}=1 / 3)$ in the expression above results the ratio being quite small (point ' $x$ '). The effect of dropping the Fierzed terms is shown by point ' + '. The figure also shows that this particular ratio is sensitive to the values used for the Wilson coefficients $c_{1}$ and $c_{2}$.

Finally, in Fig. 5.6.5 we show the ratio of the lattice annihilation amplitude to the fig8 $\pi$ amplitude. Again, the uncertainties shown for the points are large possibly due to time dependent terms of unknown size. The point on the left indicates a ratio of zero as predicted by the VS approximation in the $S U(3)$ limit (see Eq. (5.5.9)).

\subsection{Lattice Vacuum Saturation}

Return to figures 5.3.1 through 5.3.3 and observe the overall magnitude of the correlations functions in the figures. Neglecting effects due to time dependencies, take the "plateau" at large time-slices as a measure of the corresponding amplitude. Note that The fig $8 \pi_{1}$ function $\left(\mathcal{O}_{1}\right.$ operator) is larger in magnitude than fig $8 \pi_{2}$. For the fig $8 \mathrm{~K}$ functions, fig $8 \mathrm{~K}_{2}$ has the larger magnitude. According to Sect. 4.5.4 these observations are qualitatively in agreement with the leading order behavior expected in the $1 / \mathrm{N}$ expansion (see Table 4.5.1). The annihilation functions do not seem to agree with the $1 / \mathrm{N}$ expectations since both fig8an $\mathrm{an}_{1}$ and fig8 $_{2}$ are approximately equal in magnitude.

The vacuum saturation expressions for the fig8 amplitudes discussed in Sect. 5.5 determine the relative contributions of the factorizable pieces of each amplitude. Consider the VS expressions for fig8 $\pi$ (Eqs. 5.5.6). In the VS approximation the magnitude of the fig $8 \pi_{2}$ amplitude is one third of the fig $8 \pi_{1}$ amplitude and the two amplitudes have the same sign. Note that in Fig. 5.3.1 although the 
fig $8 \pi_{2}$ amplitude has roughly expected order of magnitude it has the opposite sign relative to fig $8 \pi_{1}$. Taking the ratio of the lattice fig $8 \pi_{2}$ to fig $8 \pi_{1}$ functions yields the value $-.37(8)$.

The discrepancy between the vacuum saturation prediction and the four-point function calculation on the lattice may be due to non-factorizable contributions that are manifestly present in the lattice calculation but which are not included by the vacuum saturation calculation.

At leading order the $1 / \mathrm{N}$ expansion and vacuum saturation are the same since leading order planar diagrams are factorizable and the effect of planar gluons in the VS expression is absorbed into the definitions of form factors. Non-factorizable contributions first occur at order $1 / \mathrm{N}$ along with the factorizable contributions as represented by the Fierz term in VS.

The observation that the non-factorizable contributions cannot a priori be considered negligible compared to the factorizable contributions at order $1 / \mathrm{N}$ may then suggest that all order $1 / \mathrm{N}$ should be dropped on the basis of consistency. This is then the motivation in dropping the Fierz terms in the VS approximation.

Factorization an important tool for calculation. The lesson of the $1 / \mathrm{N}$ expansion is that it can be used to find the leading order behavior of amplitudes. At order $1 / \mathrm{N}$, the large $\mathrm{N}$ expansion warns of the approximation we make in using VS.

On the lattice we make a direct test of the vacuum saturation approximation by calculating amplitudes both from four-point functions and by implementing VS on the lattice (see Sects. 4.5.5 and 4.8.2).

Figure 5.7.1 shows the TI ratio for the fig $8 \pi_{1}$ function computed by the fourpoint function and in the VS approximation. The difference between the fourpoint function values and the VS function values appears to be consistent with 


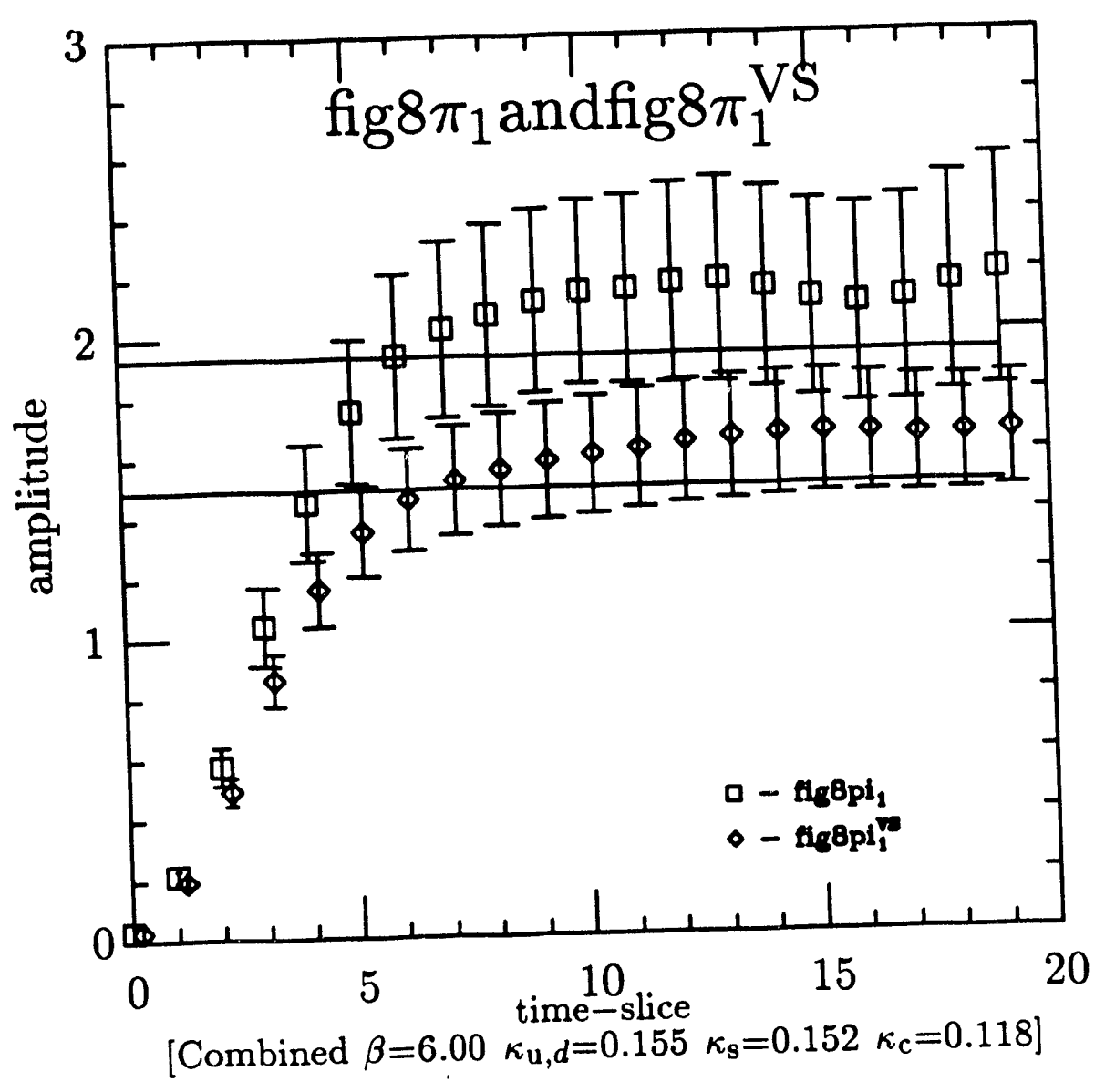

Figure 5.7.1: TI ratio for fig $8 \pi_{1}$ and the lattice vacuum saturation function fig $8 \pi_{1}^{V S}$ versus $t_{K}$. The vacuum saturation points are slightly displaced horizontally for added legibility. These correlation functions are renormalized to the continuum. The quantity fig $8 \pi_{1}$ is order 1 in the $1 / \mathrm{N}$ expansion.

deviations at the order $1 / \mathrm{N}$ level. A effect of this order of magnitude is exactly what one expects from nonfactorizable effects (which will include final state interactions).

Next consider fig $8 \pi_{2}$ which according to the $1 / \mathrm{N}$ expansion is to leading order $1 / \mathrm{N}$. Figure 5.7.2 indeed does show that the difference between the VS values and the four-point function values are of the same order of magnitude as the functions 


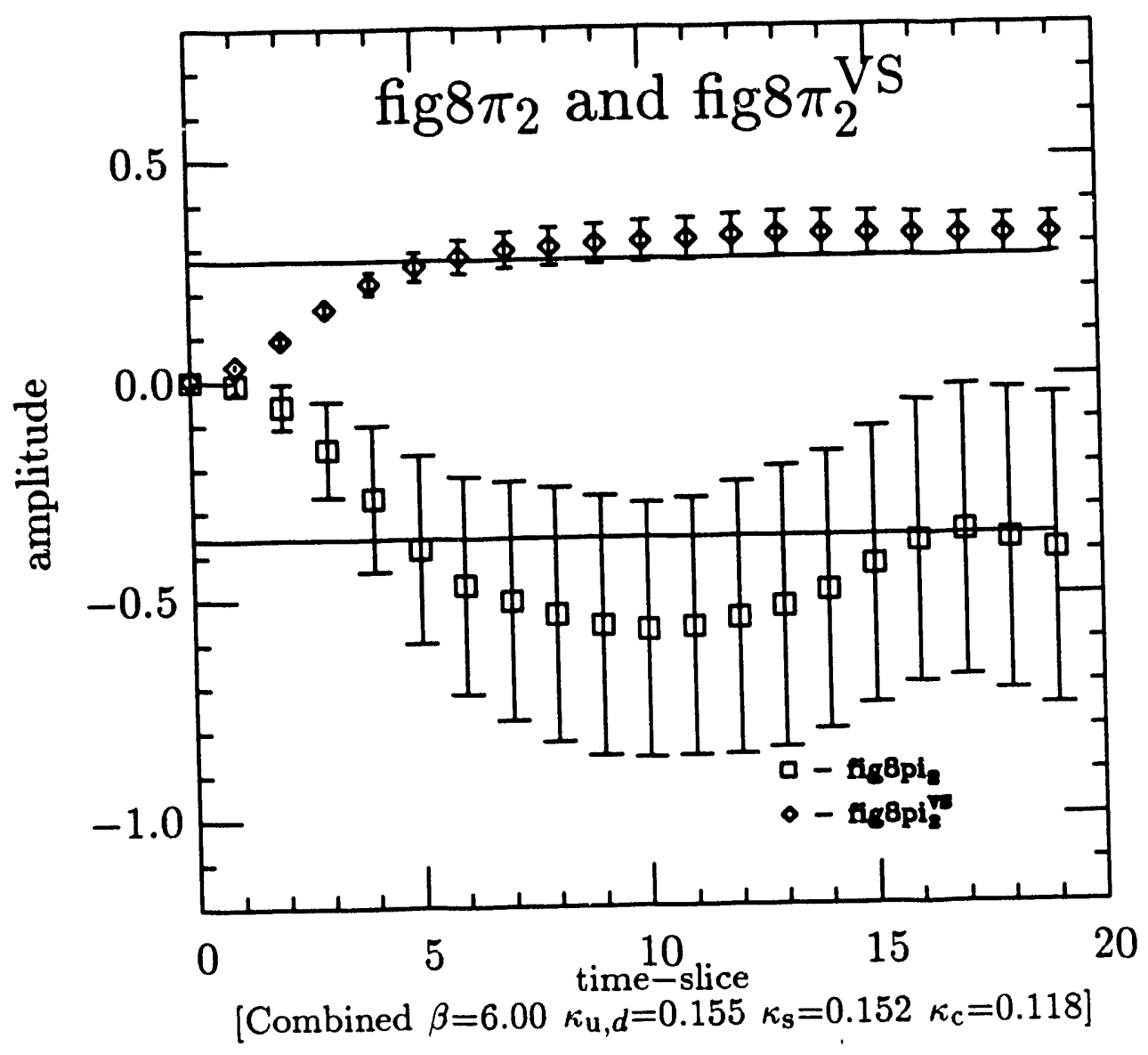

Figure 5.7.2: $\mathrm{TI}$ ratio for fig $8 \pi_{2}$ and the lattice vacuum saturation function fig $8 \pi_{2}^{V S}$ versus $t_{K}$. These correlation functions are renormalized to the continuum. The quantity fig $8 \pi_{2}$ is order $1 / \mathrm{N}$ in the $1 / \mathrm{N}$ expansion.

themselves. Note that the vacuum saturation value even has the opposite sign relative to the four-point function value.

As a check, note that the vacuum saturation results fig $8 \pi_{1}^{V S}$ and fig $8 \pi_{2}^{V S}$ have the same relative sign and approximately the correct relative magnitudes expected by Eq. (5.5.6).

This discussion focused upon fig $8 \pi$. The observations above were also found to hold for fig8K and fig8an. 
amplitudes. Note that even when subject to the at rest constraint, lattice results may still serve as a valuable check upon continuum models.

Quantities such as the ratio of the color suppressed spectator amplitude to the spectator amplitude, the ratio of the annihilation amplitude to the spectator amplitude and the ratio of $\mathcal{A}_{1 / 2}$ to $\mathcal{A}_{3 / 2}$ amplitudes calculated on the lattice were compared to the predictions of the VS model. Vacuum saturation predictions are shown for both the naive vacuum saturation approximation (keeping order $1 / \mathrm{N}$ terms) as well as with order $1 / \mathrm{N}$ terms dropped. Dropping order $1 / \mathrm{N}$ terms is motivated by the $1 / \mathrm{N}$ expansion ${ }^{5}$ and the model of $\mathrm{Stech}^{4}$ et al. At present, the lattice results allow only qualitative comparisons with continuum models.

Vacuum saturation was implemented on the lattice as a test of factorization and of the VS approximation. The lattice results show qualitative agreement of four-point function amplitudes and VS amplitudes for amplitudes estimated to be order 1 in the $1 / \mathrm{N}$ expansion. Lattice vacuum saturation amplitudes for amplitudes predicted to be order $1 / \mathrm{N}$ seemed to differ markedly from the four-point function result. According to $1 / \mathrm{N}$ expansion expectations, at order $1 / \mathrm{N}$ both factorizable and non-factorizable contributions are present. These non-factorizable contributions are represented in the four-point function result while absent from the lattice VS result. These lattice results may then indicate that non-factorizable contributions present at order $1 / \mathrm{N}$ are significant. 


\section{Bibliography}

1. J. Ellis, M. Gaillard, D. Nanopoulos, Nucl. Phys. $\underline{B} 100,313$ (1975).

2. N. Cabibbo, L. Maiani Phys. Lett. $\underline{73 B}, 418$ (1978).

3. Particle Data Group, Phys. Lett. B239, 3.54 (1990).

4. M. Bauer, B. Stech, M. Wirbel, Z. Phys. C34, 103 (1987).

5. A. J. Buras, J.-M. Gérard, R. Rückl, Nucl. Phys. B268, 16 (1986).

6. B. Blok, M. Shifman, Yad. Fiz. $\underline{45}, 211$ (1987).

7. I. I. Bigi in CCAST Symposium on Charm Physics (1987: Peking, China), ed. M. Ye, T. Huang. New York: Gordon-Breach (1988).

8. M. Bander, D. Silverman, A. Soni, Phys. Rev. Lett. $\underline{44}, 7$ (1980) errata: $\underline{44}$, $962(1980)$.

9. B. Guberina, S. Nussinov, R. Peccei, R. Rückl, Phys. Lett. $\underline{\text { 89B }} 111$ (1979).

10. H. Lipkin, Phys. Rev. Lett. $\underline{44}, 710$ (1980).

11. MARK III Collaboration (J. Adler et al.), Phys. Rev. Lett. $\underline{60}, 89$ (1988).

12. N. Deshpande in BNL Summer Study on CP Violation 1990, ed. S. Dawson and A. Soni: Singapore, World Scientific (1991).

13. D. Choudhury, D. Indumati, A. Soni, S. Sankar, Measuring $\left|V_{u b}\right|$ via Nonleptonic Decays of $B$ Mesons, Brookhaven National Lab. preprint BNL46461.

14. MARK III Collaboration (J. Adler et al.), Phys. Lett. 196B, 107 (1987).

15. M. Creutz, Quarks, Gluons and Lattices, Cambridge: Cambridge University Press (1983).

16. M. Creutz, L. Jacobs, C. Rebbi, Phys. Rept. 95, 201 (1983).

17. A. Hasenfratz, P. Hasenfratz, Ann. Rev. of Nucl. Part. Sci. $\underline{35}, 559$ (1985). 
18. C. Itzykson, J.-M. Drouffe, Statistical Field Theory, volumes 1 and 2, Cambridge: Cambridge University Press (1989).

19. K. Wilson, Phys. Rev. D10, 2445 (1974).

20. J. Bjorken, S. Drell, Relativistic Quantum Fields, New York: McGraw-Hill (1965).

21. L. Karsten, J. Smit, Nucl. Phys. B183, 103 (1981).

22. K. Wilson in New Phenomena in Subnuclear Physics, ed. A. Zichichi. New York: Plenum Press (1977).

23. C. Bernard in From Action to Answers: Proceedings of the 1989 Theoretical Advanced Study Institute in Elementary Particle Physics, Ed. T. DeGrand and D. Toussaint, Singapore: World Scientific (1990).

24. M. K. Gaillard, B. W. Lee, Phys. Rev. Lett. $\underline{33}, 108$ (1974).

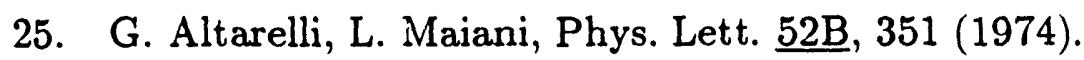

26. G. Martinelli, Phys. Lett. 141B, 395 (1984).

27. C. Bernard, A. Soni, T. Draper, Phys. Kev. D $\underline{36}, 3224$ (1987).

28. G. P. Lepage, P. Mackenzie in LATTICE 90: Proceedings of the 1990 Symposium on Lattice Field Theory, Tallahassee, Florida, ed. U. Heller et al. Amsterdam: North-Holland (1991).

29. C. Bernard, T. Draper, G. Hockney, A. Soni in LATTICE 87: Proceedings of the International Symposium on Field Theory on the Lattice, Seillac, France ed. A. Billoire et al. Amsterdam: North-Holland (1988).

30. L. Maiani, M. Testa, Phys. Lett. B245, 585 (1990).

31. V. Bernard, N. Kaiser, U. Meißner, Universität Bern preprint BUTP-91/01. This paper also appears as preprint CRN/PN-91-02. Submitted to Nucl. Phys. B.

32. M. Lüscher, Commun. Math. Phys. 105, 153 (1986). 
33. S. R. Sharpe in LATTICE 90: Proceedings of the 1990 Symposium on Lattice Field Theory, Tallahassee, Florida, ed. U. Heller et al. Amsterdam: NorthHolland (1991).

34. T. A. Draper Lattice Evaluation of Strong Corrections to Weak Matrix Elements - The $\Delta I=1 / 2$ Rule UCLA PhD dissertation (1984).

35. L. Maiani, G. Martinelli, Phys. Lett. 178B, 265 (1986).

36. C. Bernard, J. Simone, A. Soni in LATTICE 89: Proceedings of the 1989 Symposium on Lattice Field Theory, Capri Italy, ed. N. Cabibbo et al. Amsterdam: North-Holland (1990).

37. C. Bernard, J. Simone, A. Soni in LATTICE 90: Proceedings of the 1990 Symposium on Lattice Field Theory, Tallahassee, Florida, ed. U Heller et al. Amsterdam: North-Holland (1991).

38. H. Q. Ding, C. F. Baillie, G. C. Fox, Phys. Rev. D41, 2912 (1990).

39. A. Abada, Ph. Boucaud, M. Crisafulli, E. Franco, M. B. Gavela, V. J. Hill, G. Martinelli, O. Pene, C. T. Sachrajda in LATTICE 89: Proceedings of the 1989 Sympusium on Lattice. Field Theory, Capri Italy, ed. N. Cabibbo et al. Arnsterdam: North-Holland (1990).

40. NCSA HDF Calling Interfaces and Utilities National Center for Supercomputer Applications, University of Illinois at Urbana-Champaign. Sources and documentation are available via anonymous FTP to ftp.ncsa.uiuc.edu over Internet.

41. W. Press, B. Flannery, S. Teukolsky, W. Vetterling, Numerical Recipes in $C$, The Art of Scientific Programming. New York: Cambridge U'niversity Press, (1988).

42. B. Efron, SIAM Review 21,460 (1979).

43. C. Bernard, A. Soni in LATTICE 88: Proceedings of the 1988 Symposium on Lattice Field Theory, Batavia, IL, ed. A. Kronfeld, P. Mackenzie. Amsterdam: North-Holland (1989).

44. T. Morozumi in BNL Summer Study on CP Violation 1990, ed. by S. Dawson and A. Soni. Singapore: World Scientific (1991).

45. D. Aston et al., Nucl. Phys. B296, 493 (1988). 
46. S. Gottlieb, P. Mackenzie, H. Thacker, D. Weingarten, Nucl. Phys. B263, 704 (1986).

47. C. Bernard, T. Draper, G. Hockney, A. Soni, Phys. Rev. D38, 3540 (1988).

48. We thank Gustavo Burdman for suggesting the use of vacuum saturation to understand the pion mass dependence of the $D \rightarrow K \pi$ amplitudes..

49. A. El-Khadra, Lattice Calculation of Meson Form Factors for Semi-leptonic Decays, Ph. D. dissertation, UCLA (1989).

50. A. D. Kennedy, B. J. Pendleton, Phys. Lett. 156B, 393 (1985).

51. M. Creutz, Phys. Rev. D21, 2308 (1980).

52. N. Cabibbo, E. Marinari, Phys. Lett. 119B, 387 (1982).

53. G. M. Hockney. In LATTICE 89: Proceedings of the 1989 Symposium on Lattice Field Theory, Capri Italy, ed. N. Cabibbo et al. Amsterdam: NorthHolland (1990).

54. Y. Oyanagi, Comp. Phys. Comm. $\underline{42}, 333,(1986)$. 


\section{Appendix I.}

\section{Tabulated Results and Graphs: $\beta=\mathbf{6 . 0}$}

\section{I.1. Pion Masses and $Z$ Values}

\begin{tabular}{|c|c|c|c|c|c|c|}
\hline $\begin{array}{c}\text { Lattice } \\
\text { (configs) }\end{array}$ & t Range & $Z_{\pi}$ & $m_{\pi}$ & Covar. & Coeff. & $\frac{\chi^{2}}{n_{d}}$ \\
\hline \multicolumn{7}{|c|}{$\kappa_{u, d}=.155$} \\
\hline $\begin{array}{c}16^{3} \times 39 \\
(19)\end{array}$ & {$[12,16]$} & $0.465(29)$ & $0.318(9)$ & $\begin{array}{c}1 \\
0.857\end{array}$ & $\begin{array}{c}0.857 \\
1\end{array}$ & $\frac{0.34}{3}$ \\
\hline $\begin{array}{c}24^{3} \times 39 \\
(8)\end{array}$ & {$[12,16]$} & $0.478(19)$ & $0.316(5)$ & $\begin{array}{c}1 \\
0.707\end{array}$ & $\begin{array}{c}0.707 \\
1\end{array}$ & $\frac{2.8}{3}$ \\
\hline $\begin{array}{l}\text { Combined } \\
\text { (27) }\end{array}$ & {$[12,16]$} & $0.471(21)$ & $0.317(6)$ & $\begin{array}{c}1 \\
0.831\end{array}$ & $\begin{array}{c}0.831 \\
1\end{array}$ & $\frac{0.70}{3}$ \\
\hline \multicolumn{7}{|c|}{$\kappa_{u, d}=.154$} \\
\hline $\begin{array}{c}16^{3} \times 39 \\
(19)\end{array}$ & {$[12,16]$} & $0.502(26)$ & $0.381(7)$ & $\begin{array}{c}1 \\
0.867\end{array}$ & $\begin{array}{c}0.867 \\
1\end{array}$ & $\frac{0.18}{3}$ \\
\hline $\begin{array}{c}24^{3} \times 39 \\
(8)\end{array}$ & {$[12,16]$} & $0.497(17)$ & $0.373(6)$ & $\begin{array}{c}1 \\
0.818\end{array}$ & $\begin{array}{c}0.818 \\
1\end{array}$ & $\frac{1.1}{3}$ \\
\hline $\begin{array}{l}\text { Combined } \\
(27)\end{array}$ & {$[12,16]$} & $0.501(18)$ & $0.379(5)$ & $\begin{array}{c}1 \\
0.848\end{array}$ & $\begin{array}{c}0.848 \\
1\end{array}$ & $\frac{0.77}{3}$ \\
\hline \multicolumn{7}{|c|}{$\kappa_{\mathrm{u}, d}=.152$} \\
\hline $\begin{array}{c}16^{3} \times 39 \\
(19)\end{array}$ & {$[12,16]$} & $0.575(25)$ & $0.489(6)$ & $\begin{array}{c}1 \\
0.903\end{array}$ & $\begin{array}{c}0.903 \\
1\end{array}$ & $\frac{1.2}{3}$ \\
\hline $\begin{array}{c}24^{3} \times 39 \\
(8) \\
\end{array}$ & {$[12,16]$} & $0.569(19)$ & $0.483(6)$ & $\begin{array}{c}1 \\
0.912 \\
\end{array}$ & $\begin{array}{c}0.912 \\
1 \\
\end{array}$ & $\frac{1.2}{3}$ \\
\hline $\begin{array}{c}\text { Combined } \\
(27)\end{array}$ & {$[12,16]$} & $0.574(18)$ & $0.487(5)$ & $\begin{array}{c}1 \\
0.897\end{array}$ & $\begin{array}{c}0.897 \\
1\end{array}$ & $\frac{1.8}{3}$ \\
\hline
\end{tabular}

Table I.1.1: Pion two-point function fits for the $\beta=6.0$ lattices. The model function fitted is $f(t)=\frac{Z^{2}}{2 m} e^{-m t}$. 


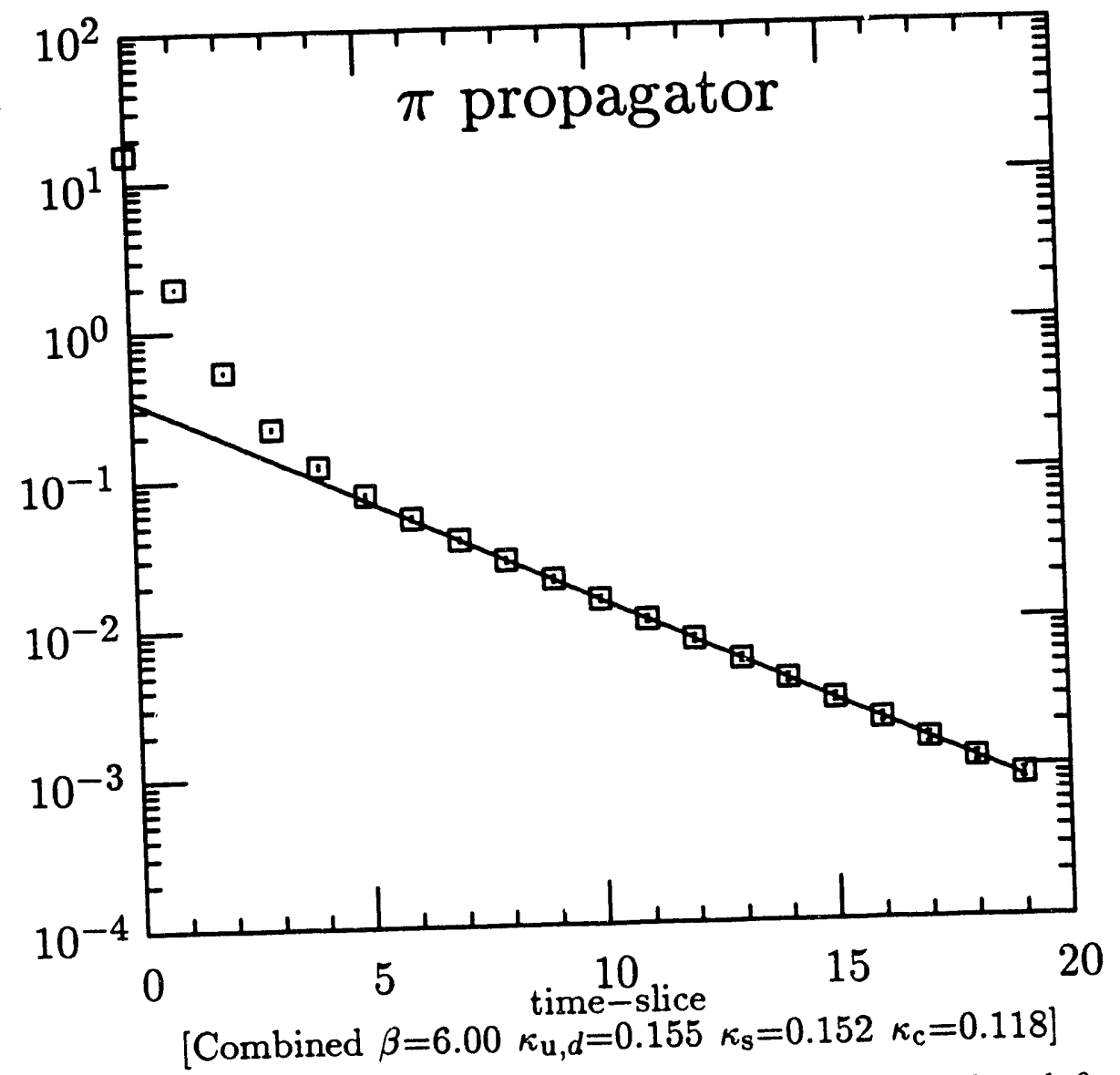

Figure I.1.1: Pion propagator with $\kappa_{u, d}=.155$ for the combined $\beta=6.0$ lattices. 


\section{I.2. Kaon Masses and $Z$ Values}

\begin{tabular}{|c|c|c|c|c|c|c|}
\hline $\begin{array}{l}\text { Lattice } \\
\text { (configs) }\end{array}$ & t Range & $Z_{K}$ & $m_{K}$ & Covar. & Coeff. & $\frac{\chi^{2}}{n_{d}}$ \\
\hline \multicolumn{7}{|c|}{$\kappa_{u, d}=.155, \kappa_{s}=.152$} \\
\hline $\begin{array}{c}16^{3} \times 39 \\
(19)\end{array}$ & {$[12,16]$} & $0.513(25)$ & $0.411(7)$ & $\begin{array}{c}1 \\
0.857 \\
\end{array}$ & $\begin{array}{c}0.857 \\
1 \\
\end{array}$ & $\frac{0.37}{3}$ \\
\hline $\begin{array}{c}24^{3} \times 39 \\
(8)\end{array}$ & {$[12,16]$} & $0.503(17)$ & $0.402(6)$ & $\begin{array}{c}1 \\
0.824 \\
\end{array}$ & $\begin{array}{c}0.824 \\
1 \\
\end{array}$ & $\frac{1.0}{3}$ \\
\hline $\begin{array}{c}\text { Combined } \\
(27)\end{array}$ & {$[12,16]$} & $0.510(18)$ & $0.408(5)$ & $\begin{array}{c}1 \\
0.844\end{array}$ & $\begin{array}{c}0.844 \\
1\end{array}$ & $\frac{1.1}{3}$ \\
\hline \multicolumn{7}{|c|}{$\kappa_{u, d}=154, \kappa_{3}=.152$} \\
\hline $\begin{array}{c}16^{3} \times 39 \\
(19)\end{array}$ & {$[12,16]$} & $0.535(25)$ & $0.437(7)$ & $\begin{array}{c}1 \\
0.881 \\
\end{array}$ & $\begin{array}{c}0.881 \\
1 \\
\end{array}$ & $\frac{0.64}{3}$ \\
\hline $\begin{array}{c}24^{3} \times 39 \\
(8) \\
\end{array}$ & {$[12,16]$} & $0.527(17)$ & $0.430(6)$ & $\begin{array}{c}1 \\
0.878 \\
\end{array}$ & $\begin{array}{c}0.878 \\
1 \\
\end{array}$ & $\frac{0.77}{3}$ \\
\hline $\begin{array}{l}\text { Combined } \\
(27) \\
\end{array}$ & {$[12,16]$} & $0.533(18)$ & $0.435(5)$ & $\begin{array}{c}1 \\
0.871 \\
\end{array}$ & $\begin{array}{c}0.871 \\
1\end{array}$ & $\frac{1.3}{3}$ \\
\hline \multicolumn{7}{|c|}{$\kappa_{u, d}=.152, \kappa_{s}=.152$} \\
\hline $\begin{array}{c}16^{3} \times 39 \\
(19)\end{array}$ & {$[12,16]$} & $0.575(25)$ & $0.489(6)$ & $\begin{array}{c}1 \\
0.903 \\
\end{array}$ & $\begin{array}{c}0.903 \\
1 \\
\end{array}$ & $\frac{1.2}{3}$ \\
\hline $\begin{array}{c}24^{3} \times 39 \\
(8) \\
\end{array}$ & {$[12,16]$} & $0.569(19)$ & $0.483(6)$ & $\begin{array}{c}1 \\
0.912 \\
\end{array}$ & $\begin{array}{c}0.912 \\
1 \\
\end{array}$ & $\frac{1.2}{3}$ \\
\hline $\begin{array}{c}\text { Combined } \\
(27)\end{array}$ & {$[12,16]$} & $0.574(18)$ & $0.487(5)$ & $\begin{array}{c}1 \\
0.897 \\
\end{array}$ & $\begin{array}{c}0.897 \\
1\end{array}$ & $\frac{1.8}{3}$ \\
\hline
\end{tabular}

Table 1.2.1: Kaon two-point function fits for the $\beta=6.0$ lattices. The model function fitted is $f(t)=\frac{Z^{2}}{2 m} e^{-m t}$. 


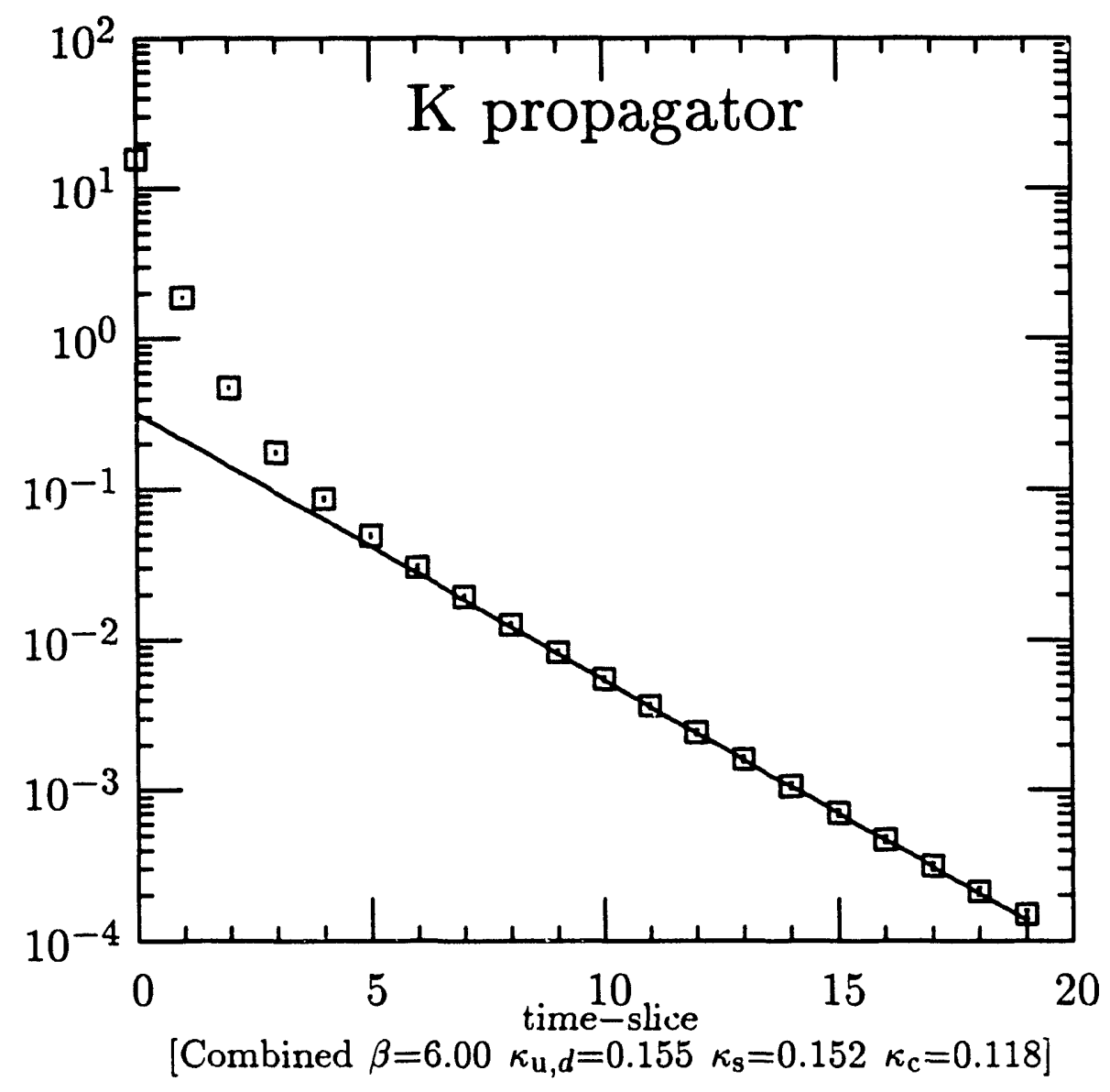

Figure I.2.1: Kaon propagator with $\kappa_{u, i}=.155, \kappa_{s}=.152$ for the combined $\beta=6.0$ lattices. 


\subsection{Masses and $Z$ Values}

\begin{tabular}{|c|c|c|c|c|c|c|}
\hline $\begin{array}{c}\text { Lattice } \\
\text { (configs) }\end{array}$ & t Range & $Z_{D}$ & $m_{D}$ & Covar. & Coeff. & $\frac{\chi^{2}}{n_{d}}$ \\
\hline \multicolumn{7}{|c|}{$\kappa_{u, d}=.155, \kappa_{c}=.118$} \\
\hline $\begin{array}{c}16^{3} \times 39 \\
(19) \\
\end{array}$ & {$[12,16]$} & $0.777(45)$ & $1.18(1)$ & $\begin{array}{c}1 \\
0.913 \\
\end{array}$ & $\begin{array}{c}0.913 \\
1 \\
\end{array}$ & $\frac{2.9}{3}$ \\
\hline $\begin{array}{c}24^{3} \times 39 \\
(8) \\
\end{array}$ & {$[12,16]$} & $.750(44)$ & $1.17(1)$ & $\begin{array}{c}1 \\
.909 \\
\end{array}$ & $\begin{array}{c}.909 \\
1 \\
\end{array}$ & $\frac{.96}{3}$ \\
\hline $\begin{array}{c}\text { Combined } \\
(27)\end{array}$ & {$[12,16]$} & $0.770(34)$ & $1.18(1)$ & $\begin{array}{c}1 \\
0.905 \\
\end{array}$ & $\begin{array}{c}0.905 \\
1 \\
\end{array}$ & $\frac{3.8}{3}$ \\
\hline \multicolumn{7}{|c|}{$\kappa_{u, d}=.154, \kappa_{c}=.118$} \\
\hline $\begin{array}{c}16^{3} \times 39 \\
(19) \\
\end{array}$ & {$[12,16]$} & $0.821(43)$ & $1.20(1)$ & $\begin{array}{c}1 \\
0.911 \\
\end{array}$ & $\begin{array}{c}0.911 \\
1 \\
\end{array}$ & $\frac{3.5}{3}$ \\
\hline $\begin{array}{c}24^{3} \times 39 \\
(8) \\
\end{array}$ & {$[12,16]$} & $0.803(36)$ & $1.19(1)$ & $\begin{array}{c}1 \\
0.887 \\
\end{array}$ & $\begin{array}{c}0.887 \\
1 \\
\end{array}$ & $\frac{1.4}{3}$ \\
\hline $\begin{array}{c}\text { Combined } \\
(27)\end{array}$ & {$[12,16]$} & $0.821(33)$ & $1.20(1)$ & $\begin{array}{c}1 \\
0.905 \\
\end{array}$ & $\begin{array}{c}0.905 \\
1 \\
\end{array}$ & $\frac{4.6}{3}$ \\
\hline \multicolumn{7}{|c|}{$\kappa_{u, d}=.152, \kappa_{c}=.118$} \\
\hline $\begin{array}{c}16^{3} \times 39 \\
(19) \\
\end{array}$ & {$[12,16]$} & $0.909(42)$ & $1.23(1)$ & $\begin{array}{c}1 \\
0.898 \\
\end{array}$ & $\begin{array}{c}0.898 \\
1 \\
\end{array}$ & $\frac{4.4}{3}$ \\
\hline $\begin{array}{c}24^{3} \times 39 \\
(8) \\
\end{array}$ & {$[12,16]$} & $0.904(25)$ & $1.23(1)$ & $\begin{array}{c}1 \\
0.832 \\
\end{array}$ & $\begin{array}{c}0.832 \\
1 \\
\end{array}$ & $\frac{2.0}{3}$ \\
\hline $\begin{array}{l}\text { Combined } \\
(27)\end{array}$ & {$[12,16]$} & $0.915(32)$ & $1.233(5)$ & $\begin{array}{c}1 \\
0.895\end{array}$ & $\begin{array}{c}0.895 \\
1 \\
\end{array}$ & $\frac{6.0}{3}$ \\
\hline \multicolumn{7}{|c|}{$\kappa_{u, d}=.155, \kappa_{c}=.135$} \\
\hline $\begin{array}{c}24^{3} \times 39 \\
(8)\end{array}$ & {$[12,16]$} & $0.665(33)$ & $0.818(9)$ & $\begin{array}{c}1 \\
0.904 \\
\end{array}$ & $\begin{array}{c}0.904 \\
1 \\
\end{array}$ & $\frac{0.93}{3}$ \\
\hline
\end{tabular}

Table 1.3.1: $\mathrm{D}$ two-point function fits for the $\beta=6.0$ lattices. The model function fitted is $f(t)=\frac{Z^{2}}{2 m} e^{-m t}$. 


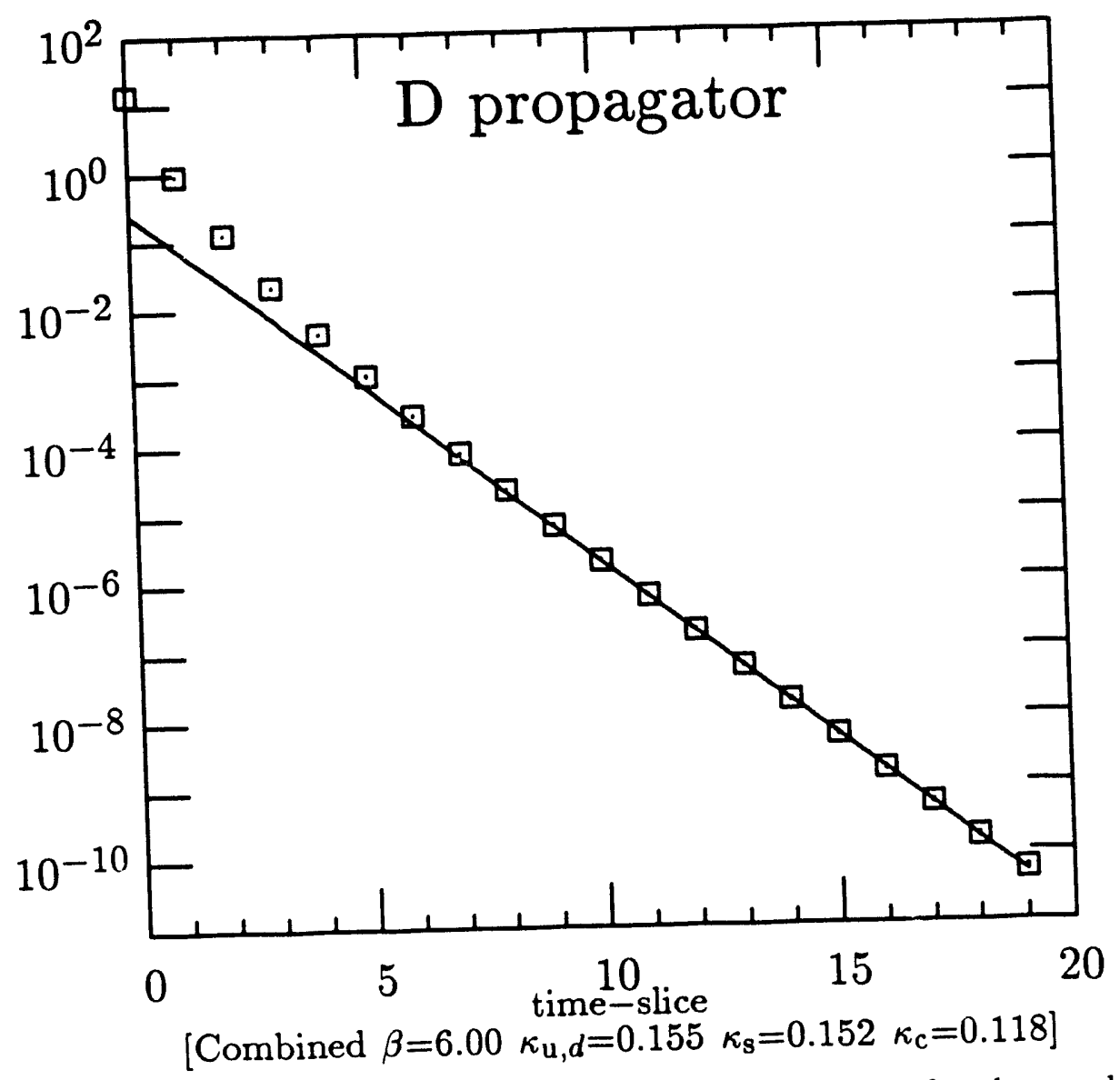

Figure I.3.1: D propagator with $\kappa_{u, d}=.155, \kappa_{c}=.118$ for the combined $\beta=6.0$ lattices. 


\section{I.4. Scalar Masses and $Z$ Values}

\begin{tabular}{|c|c|c|c|c|c|c|}
\hline $\begin{array}{c}\text { Lattice } \\
\text { (configs) }\end{array}$ & t Range & $Z_{\text {scalar }}$ & $m_{\text {scalar }}$ & Covar. Coeff. & $\frac{\chi^{2}}{n_{d}}$ \\
\hline \multicolumn{7}{|c|}{$\kappa_{u, d}=.155, \kappa_{s}=.152$} \\
\hline $\begin{array}{c}16^{3} \times 39 \\
(19)\end{array}$ & {$[9,14]$} & $0.546(309)$ & $0.731(115)$ & $\begin{array}{c}1 \\
0.985\end{array}$ & $\begin{array}{c}0.985 \\
1\end{array}$ & $\frac{2.9}{4}$ \\
\hline $\begin{array}{c}24^{3} \times 39 \\
(8)\end{array}$ & {$[9,14]$} & $0.851(143)$ & $0.789(42)$ & $\begin{array}{c}1 \\
0.945\end{array}$ & $\begin{array}{c}0.945 \\
1\end{array}$ & $\frac{3.1}{4}$ \\
\hline $\begin{array}{c}\text { Combined } \\
(27)\end{array}$ & {$[9,14]$} & $0.708(285)$ & $0.771(82)$ & $\begin{array}{c}1 \\
0.983\end{array}$ & $\begin{array}{c}0.983 \\
1\end{array}$ & $\frac{5.3}{4}$ \\
\hline $\begin{array}{c}16^{3} \times 39 \\
(19)\end{array}$ & {$[9,14]$} & $0.535(221)$ & $0.735(82)$ & $\begin{array}{c}1 \\
0.981\end{array}$ & $\begin{array}{c}0.981 \\
1\end{array}$ & $\frac{2.2}{4}$ \\
\hline $\begin{array}{c}24^{3} \times 39 \\
(8)\end{array}$ & {$[9,14]$} & $0.890(98)$ & $0.812(31)$ & $\begin{array}{c}1 \\
0.943\end{array}$ & $\begin{array}{c}0.943 \\
1\end{array}$ & $\frac{3.8}{4}$ \\
\hline $\begin{array}{c}\text { Combined } \\
(27)\end{array}$ & {$[9,14]$} & $0.643(191)$ & $0.762(59)$ & $\begin{array}{c}1 \\
0.980\end{array}$ & $\begin{array}{c}0.980 \\
1\end{array}$ & $\frac{4.3}{4}$ \\
\hline \multicolumn{1}{|c|}{$\kappa_{u, d}=.154, \kappa_{s}=.152$} \\
\hline $\begin{array}{c}16^{3} \times 39 \\
(19)\end{array}$ & {$[9,14]$} & $0.562(170)$ & $0.768(60)$ & $\begin{array}{c}1 \\
0.978\end{array}$ & $\begin{array}{c}0.978 \\
1\end{array}$ & $\frac{2.4}{4}$ \\
\hline $\begin{array}{c}24^{3} \times 39 \\
(8)\end{array}$ & {$[9,14]$} & $0.920(102)$ & $0.858(31)$ & $\begin{array}{c}1 \\
0.960\end{array}$ & $\begin{array}{c}0.960 \\
1\end{array}$ & $\frac{9.7}{4}$ \\
\hline $\begin{array}{c}\text { Combined } \\
(27)\end{array}$ & {$[9,14]$} & $0.624(144)$ & $0.787(46)$ & $\begin{array}{c}1 \\
0.978\end{array}$ & $\begin{array}{c}0.978 \\
1\end{array}$ & $\frac{3.8}{4}$ \\
\hline
\end{tabular}

Table 1.4.1: Scalar two-point function fits for the $\beta=6.0$ lattices. The model function fitted is $f(t)=\frac{Z^{2}}{2 m} e^{-m t}$. 


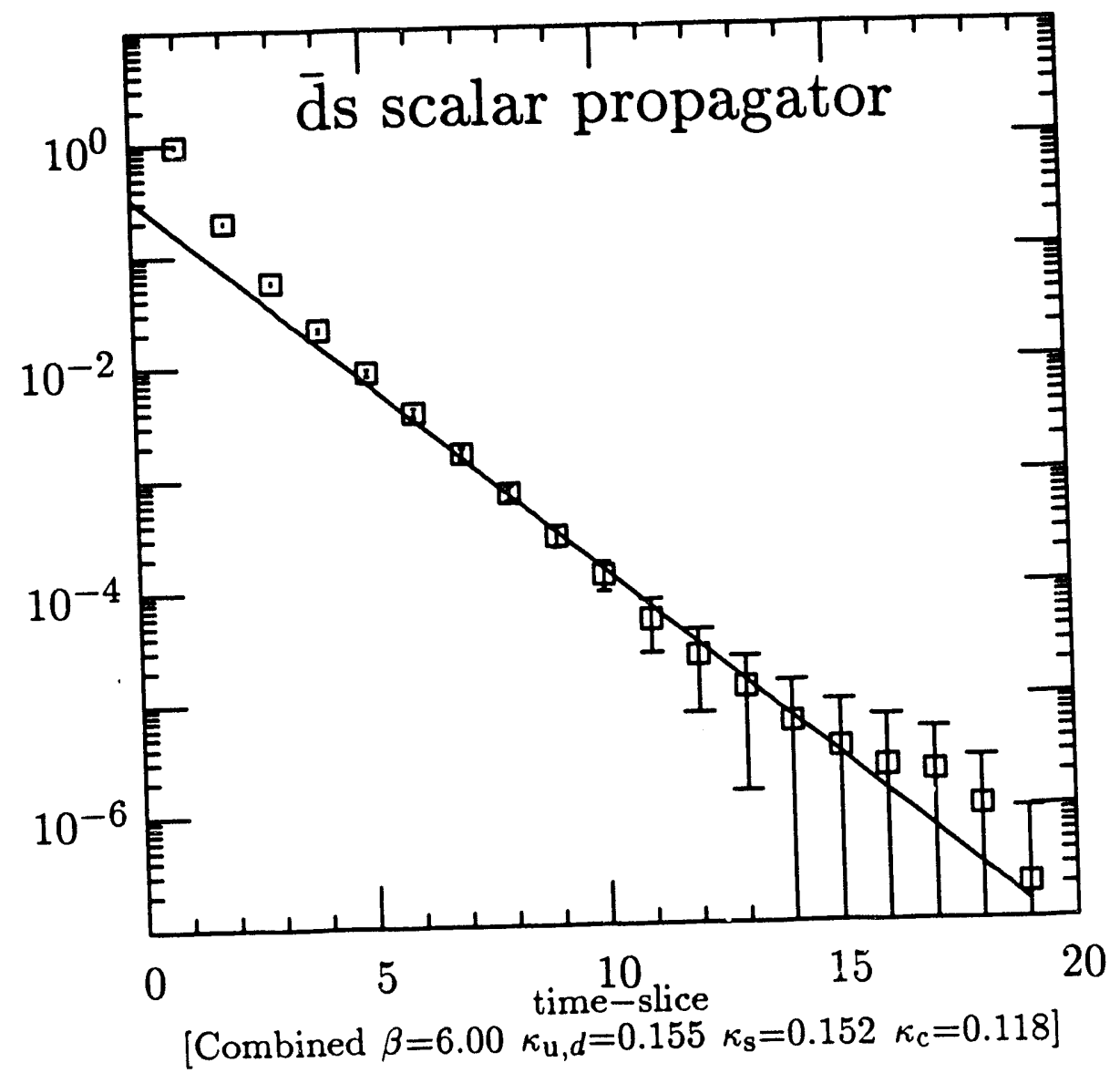

Figure I.4.1: Scalar propagator with $\kappa_{u, d}=.155, \kappa_{s}=.152$ for the combined $\beta=6.0$ lattices. 


\section{I.5. Srn Mass Differences}

\begin{tabular}{|c|c|c|c|c|c|c|}
\hline $\begin{array}{c}\text { Lattice } \\
\text { (configs) }\end{array}$ & t Range & $c_{0}$ & $\delta m$ & Covar. & Coeff. & $\frac{\chi^{2}}{n_{d}}$ \\
\hline \multicolumn{7}{|c|}{$\kappa_{\mathrm{u}, d}=.155, \kappa,=.152$} \\
\hline $\begin{array}{c}16^{3} \times 39 \\
(19) \\
\end{array}$ & {$[9,14]$} & $1.7(16)$ & $0.0095(1119)$ & $\begin{array}{c}1 \\
-0.980 \\
\end{array}$ & $\begin{array}{c}-0.980 \\
1 \\
\end{array}$ & $\frac{2.7}{4}$ \\
\hline $\begin{array}{c}24^{3} \times 39 \\
(8)\end{array}$ & {$[9,14]$} & $4.5(13)$ & $-0.087(39)$ & $\begin{array}{c}1 \\
-0.949\end{array}$ & $\begin{array}{c}-0.949 \\
1\end{array}$ & $\frac{2.9}{4}$ \\
\hline $\begin{array}{c}\text { Combined } \\
(27)\end{array}$ & {$[9,14]$} & $2.7(18)$ & $-0.038(79)$ & $\begin{array}{c}1 \\
-0.974 \\
\end{array}$ & $\begin{array}{c}-0.974 \\
1 \\
\end{array}$ & $\frac{5.1}{4}$ \\
\hline \multicolumn{7}{|c|}{$\kappa_{u, d}=.154, \kappa_{s}=.152$} \\
\hline $\begin{array}{c}16^{3} \times 39 \\
(19) \\
\end{array}$ & {$[9,14]$} & $1.6(12)$ & $0.091(84)$ & $\begin{array}{c}1 \\
-0.978 \\
\end{array}$ & $\begin{array}{c}-0.978 \\
1 \\
\end{array}$ & $\frac{2.0}{4}$ \\
\hline $\begin{array}{c}24^{3} \times 39 \\
(8) \\
\end{array}$ & {$[9,14]$} & $3.8(6)$ & $0.013(26)$ & $\begin{array}{c}1 \\
-0.960 \\
\end{array}$ & $\begin{array}{c}-0.960 \\
1 \\
\end{array}$ & $\frac{3.9}{4}$ \\
\hline $\begin{array}{c}\text { Combined } \\
(27)\end{array}$ & {$[9,14]$} & $2.3(12)$ & $0.059(59)$ & $\begin{array}{c}1 \\
-0.972 \\
\end{array}$ & $\begin{array}{c}-0.972 \\
1 \\
\end{array}$ & $\frac{3.9}{4}$ \\
\hline \multicolumn{7}{|c|}{$\kappa_{u, d}=.152, \kappa_{s}=.152$} \\
\hline $\begin{array}{c}16^{3} \times 39 \\
(19)\end{array}$ & {$[9,14]$} & $1.6(8)$ & $0.22(6)$ & $\begin{array}{c}1 \\
-0.974 \\
\end{array}$ & $\begin{array}{c}-0.974 \\
1 \\
\end{array}$ & $\frac{2.5}{4}$ \\
\hline $\begin{array}{c}24^{3} \times 39 \\
(8)\end{array}$ & {$[9,14]$} & $2.6(5)$ & $0.18(2)$ & $\begin{array}{c}1 \\
-0.947 \\
\end{array}$ & $\begin{array}{c}-0.947 \\
1 \\
\end{array}$ & $\frac{9.9}{4}$ \\
\hline $\begin{array}{c}\text { Combined } \\
(27)\end{array}$ & {$[9,14]$} & $1.8(7)$ & $0.20(5)$ & $\begin{array}{c}1 \\
-0.972\end{array}$ & $\begin{array}{c}-0.972 \\
1\end{array}$ & $\frac{3.4}{4}$ \\
\hline
\end{tabular}

Table I.5.1: Fit to the ratio $G_{\text {scalar }}^{(2)} /\left(G_{\pi}^{(2)} G_{K}^{(2)}\right)$ versus $t$. The model function fitted is $f(t)=c_{0} e^{\delta m t}$. From the asymptotic behavior of the propagators it is expected that $\delta m=-\left(m_{\text {scalar }}-m_{K}-m_{\pi}\right)$. 


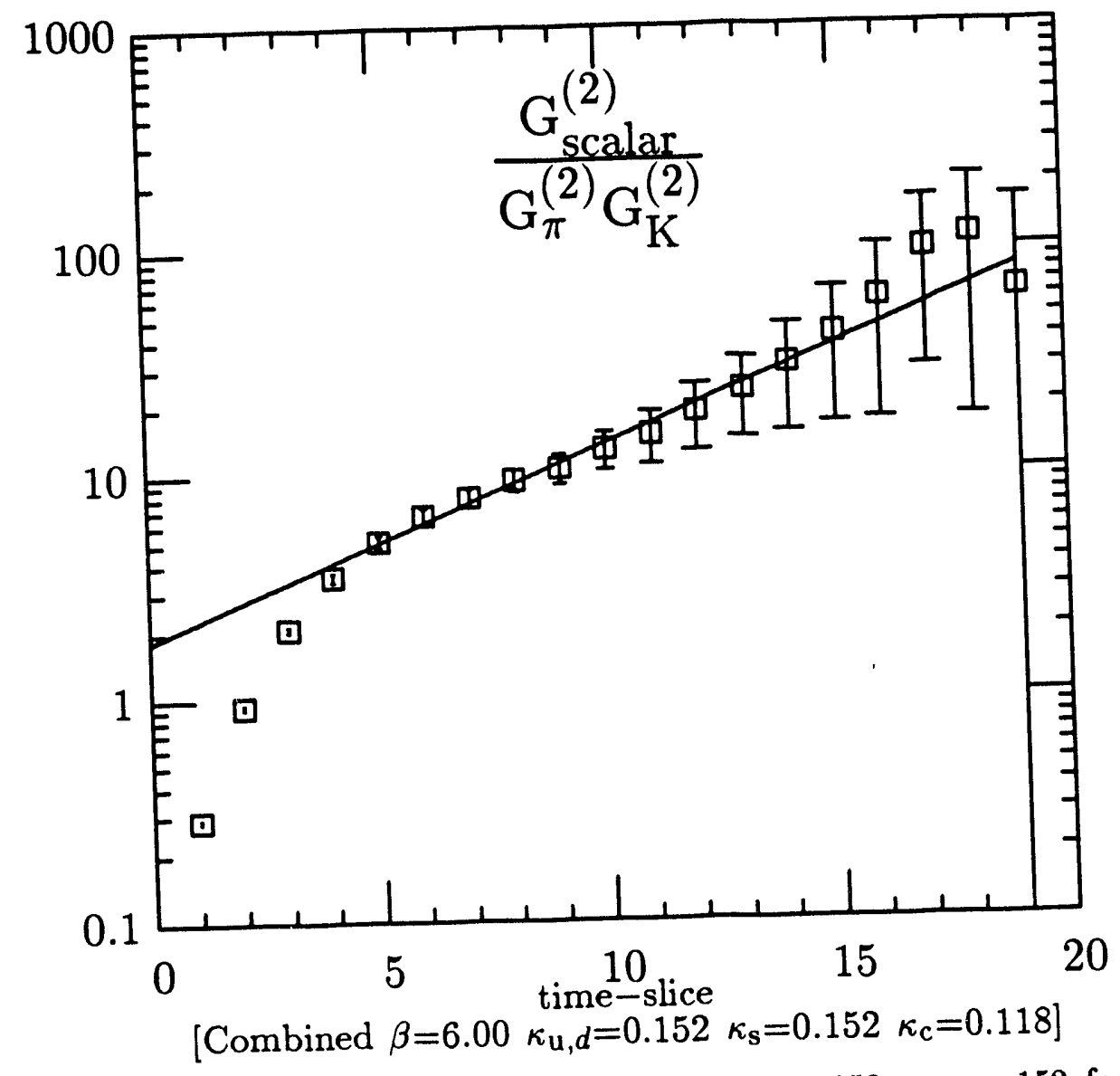

Figure I.5.1: Ratio of propagators with $\kappa_{u, d}=.152, \kappa_{s}=.152$ for the combined $\beta=6.0$ lattices. 


\section{I.6. $\mathcal{A}(\mathrm{fig} 8 \pi)$}

\begin{tabular}{|c|c|c|c|c|}
\hline $\begin{array}{l}\text { Lattice } \\
\text { (configs) }\end{array}$ & $\begin{array}{l}\text { Model } \\
\text { t Range }\end{array}$ & $\begin{array}{l}a_{1} \\
a_{2} \\
a_{3} \\
\end{array}$ & Covar. Coeff. & $\frac{\chi^{2}}{n_{d}}$ \\
\hline \multicolumn{5}{|c|}{$\kappa_{u, d}=.155, \kappa_{s}=.152, \kappa_{c}=.118$} \\
\hline $\begin{array}{c}\text { Combiried } \\
(27)\end{array}$ & $\begin{array}{c}I \\
{[12,16]}\end{array}$ & $\begin{array}{c}1.3(2) \\
0.0^{*} \\
0.0^{*} \\
\end{array}$ & $\begin{array}{lll}1 & 0 & 0 \\
0 & 0 & 0 \\
0 & 0 & 0 \\
\end{array}$ & $\frac{3.2}{4}$ \\
\hline \multicolumn{5}{|c|}{$\kappa_{u, d}=.154, \kappa_{s}=.152, \kappa_{c}=.118$} \\
\hline $\begin{array}{l}\text { Combined } \\
(27)\end{array}$ & $\begin{array}{c}I \\
{[12,16]}\end{array}$ & $\begin{array}{c}1.5(2) \\
0.0^{*} \\
0.0^{*} \\
\end{array}$ & $\begin{array}{lll}1 & 0 & 0 \\
0 & 0 & 0 \\
0 & 0 & 0 \\
\end{array}$ & $\frac{1.4}{4}$ \\
\hline \multicolumn{5}{|c|}{$\kappa_{u, d}=.152, \kappa_{s}=.152, \kappa_{c}=.118$} \\
\hline $\begin{array}{l}\text { Combined } \\
\quad(27)\end{array}$ & $\begin{array}{c}I \\
{[12,16]}\end{array}$ & $\begin{array}{c}1.5(1) \\
0.0^{*} \\
0.0^{*}\end{array}$ & $\begin{array}{lll}1 & 0 & 0 \\
0 & 0 & 0 \\
0 & 0 & 0\end{array}$ & $\frac{2.3}{4}$ \\
\hline
\end{tabular}

Table I.6.1: Fits to TI ratio for fig8 $\pi^{\text {cont }}$ versus $t_{K}$ for the $\beta=6.0$ lattices. Model Function I is $f(t)=a_{1}+a_{2} t+a_{3} t^{2}$. Model Function II is $f(t)=$ $a_{1}+a_{2} e^{a_{3} t}$. Parameters that are held fixed are followed by an asterisk. 


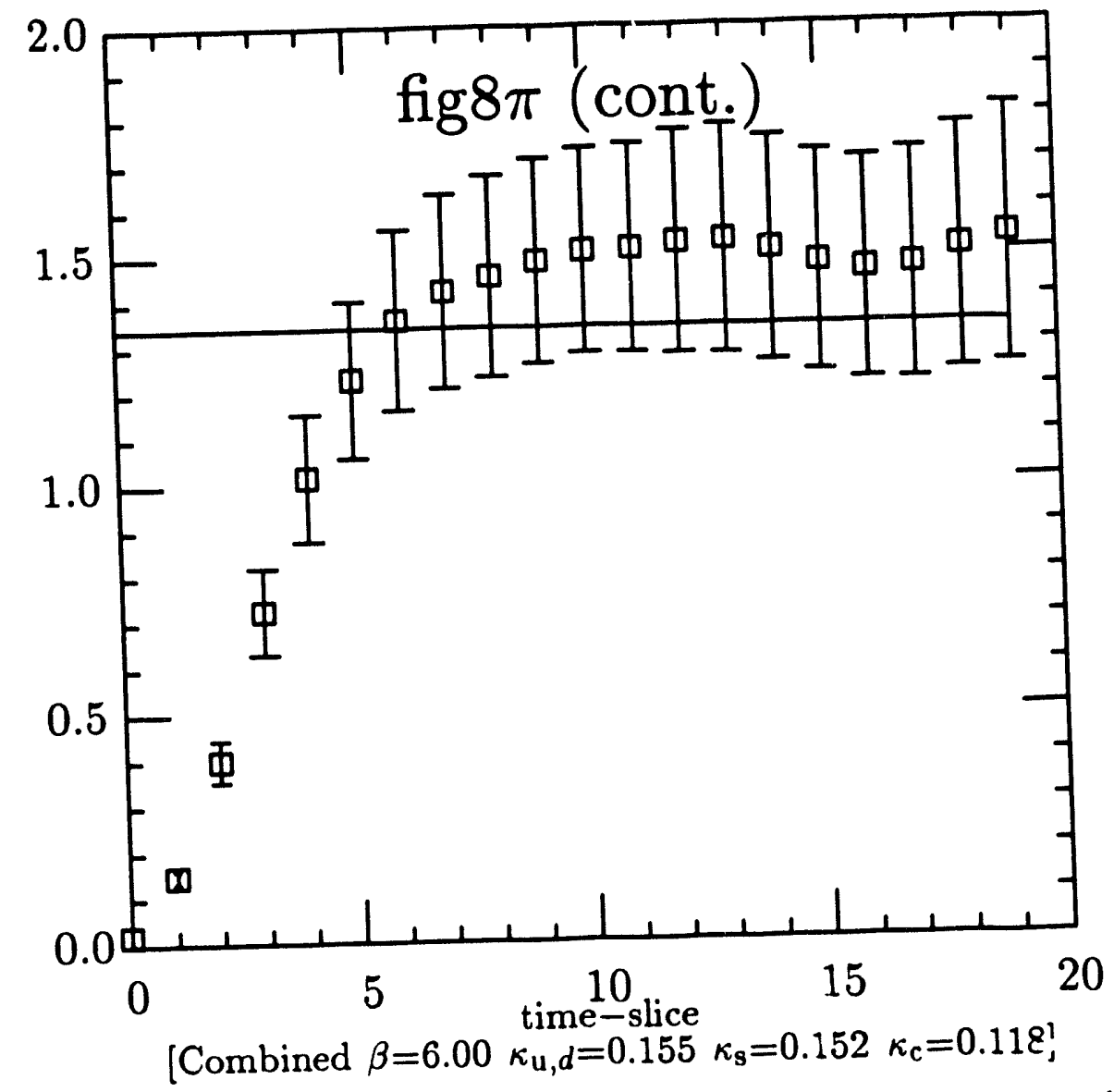

Figure 1.6.1: Fit to the TI ratio for fig $8 \pi^{\text {cont }}$ versus $t_{K}$ for the combined $\beta=6.0$ lattices. 


\section{I.7. $\mathcal{A}($ flg8K)}

\begin{tabular}{|c|c|c|c|c|}
\hline $\begin{array}{l}\text { Lattice } \\
\text { (configs) }\end{array}$ & $\begin{array}{l}\text { Model } \\
\text { t Range }\end{array}$ & $\begin{array}{l}a_{1} \\
a_{2} \\
a_{3} \\
\end{array}$ & Covar. Coeff. & $\frac{\chi^{2}}{n_{d}}$ \\
\hline \multicolumn{5}{|c|}{$\kappa_{u, d}=.155, \kappa_{s}=.152, \kappa_{c}=.118$} \\
\hline $\begin{array}{c}\text { Combined } \\
\text { (27) }\end{array}$ & $\begin{array}{c}I \\
{[12,16]}\end{array}$ & $\begin{array}{c}-0.54(19) \\
0.0^{*} \\
0.0^{*} \\
\end{array}$ & $\begin{array}{lll}1 & 0 & 0 \\
0 & 0 & 0 \\
0 & 0 & 0 \\
\end{array}$ & $\frac{2.3}{4}$ \\
\hline \multicolumn{5}{|c|}{$\kappa_{u, d}=.154, \kappa_{,}=.152, \kappa_{c}=.118$} \\
\hline $\begin{array}{l}\text { Combined } \\
(27)\end{array}$ & $\begin{array}{c}I \\
{[12,16]}\end{array}$ & $\begin{array}{c}-0.53(15) \\
0.0^{\circ} \\
0.0^{\circ} \\
\end{array}$ & $\begin{array}{lll}1 & 0 & 0 \\
0 & 0 & 0 \\
0 & 0 & 0 \\
\end{array}$ & $\frac{2.1}{4}$ \\
\hline \multicolumn{5}{|c|}{$\kappa_{u, d}=.152, \kappa_{,}=.152, \kappa_{c}=.118$} \\
\hline $\begin{array}{l}\text { Combined } \\
(27)\end{array}$ & $\begin{array}{c}I \\
{[12,16]}\end{array}$ & $\begin{array}{c}-0.40(9) \\
0.0^{*} \\
0.0^{*}\end{array}$ & $\begin{array}{lll}1 & 0 & 0 \\
0 & 0 & 0 \\
0 & 0 & 0\end{array}$ & $\frac{2.3}{4}$ \\
\hline
\end{tabular}

Table I.7.1: Fits to TI ratio for fig8K $\mathrm{K}^{\text {cont }}$ versus $t_{K}$ for the $\beta=6.0$ lattices. Model Function I is $f(t)=a_{1}+a_{2} t+a_{3} t^{2}$. Model Function II is $f(t)=a_{1}+a_{2} e^{a_{3} t}$. Parameters that are held fixed are followed by an asterisk. 


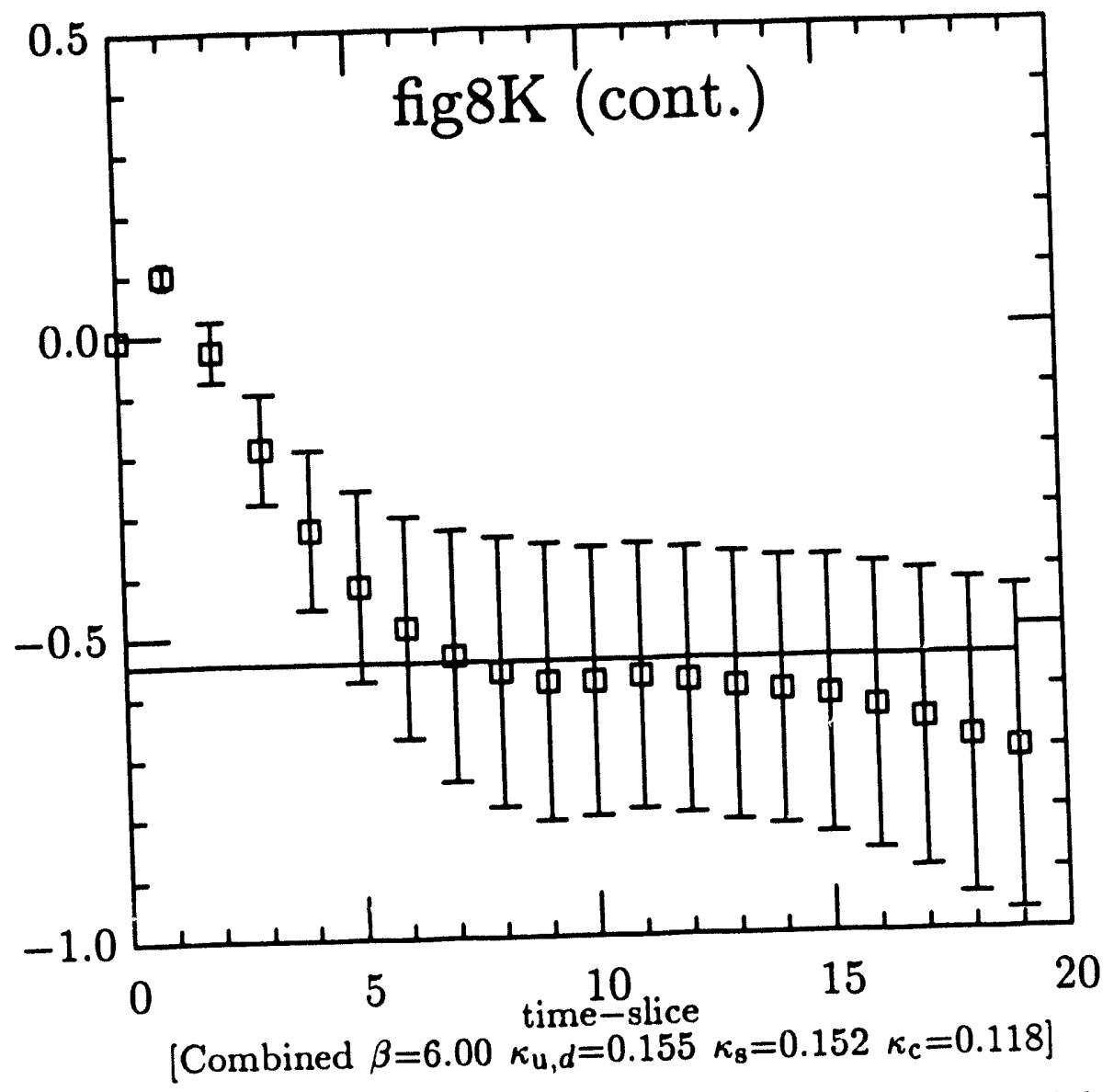

Figure 1.7.1: Fit to TI ratio for fig8 $\mathrm{K}^{\text {cont }}$ versus $t_{K}$ for the combined $\beta=6.0$ lattices. 


\section{I.8. $\mathcal{A}($ flg8an $)$}

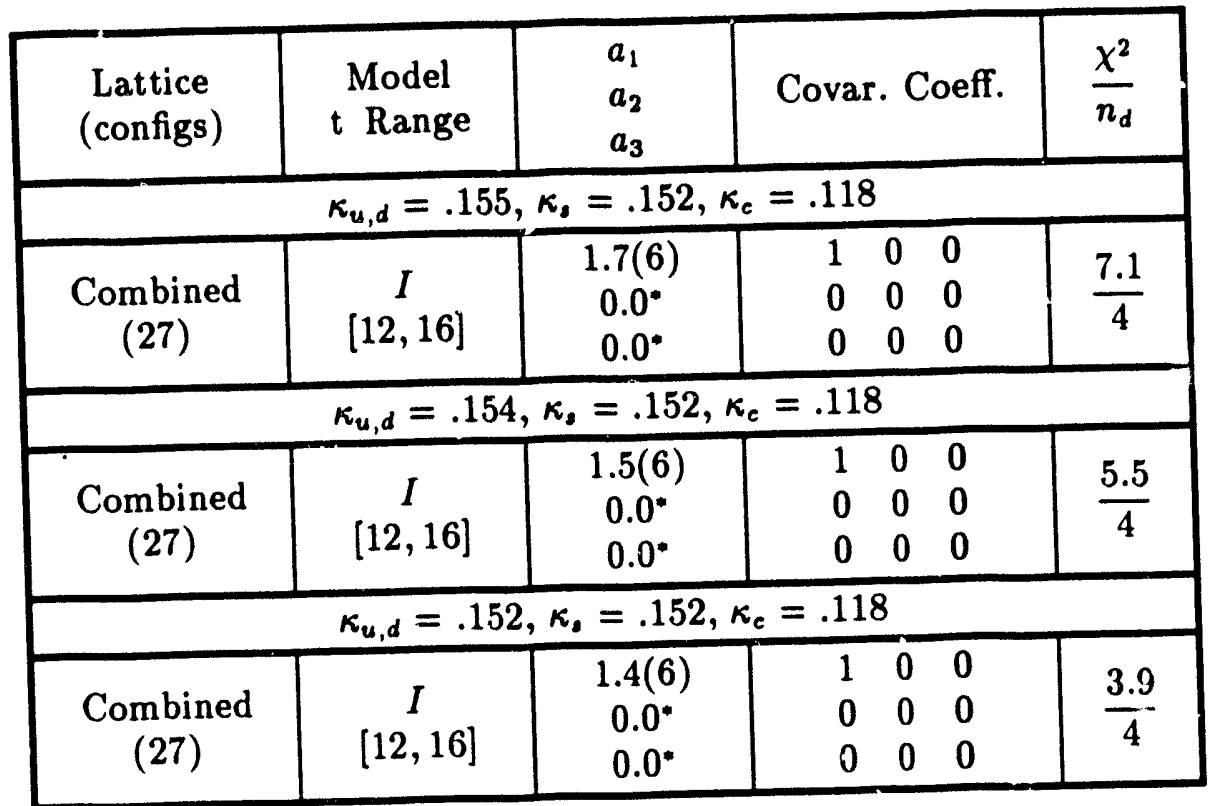

Table 1.8.1: Fits to TI ratio for fig8an ${ }^{\text {cont }}$ versus $t_{K}$ for the $\beta=6.0$ lattices. Model Function I is $f(t)=a_{1}+a_{2} t+a_{3} t^{2}$. Model Function II is $f(t)=a_{1}+a_{2} e^{a_{3} t}$. Parameters that are held fixed are foliowed by an asterisk. 


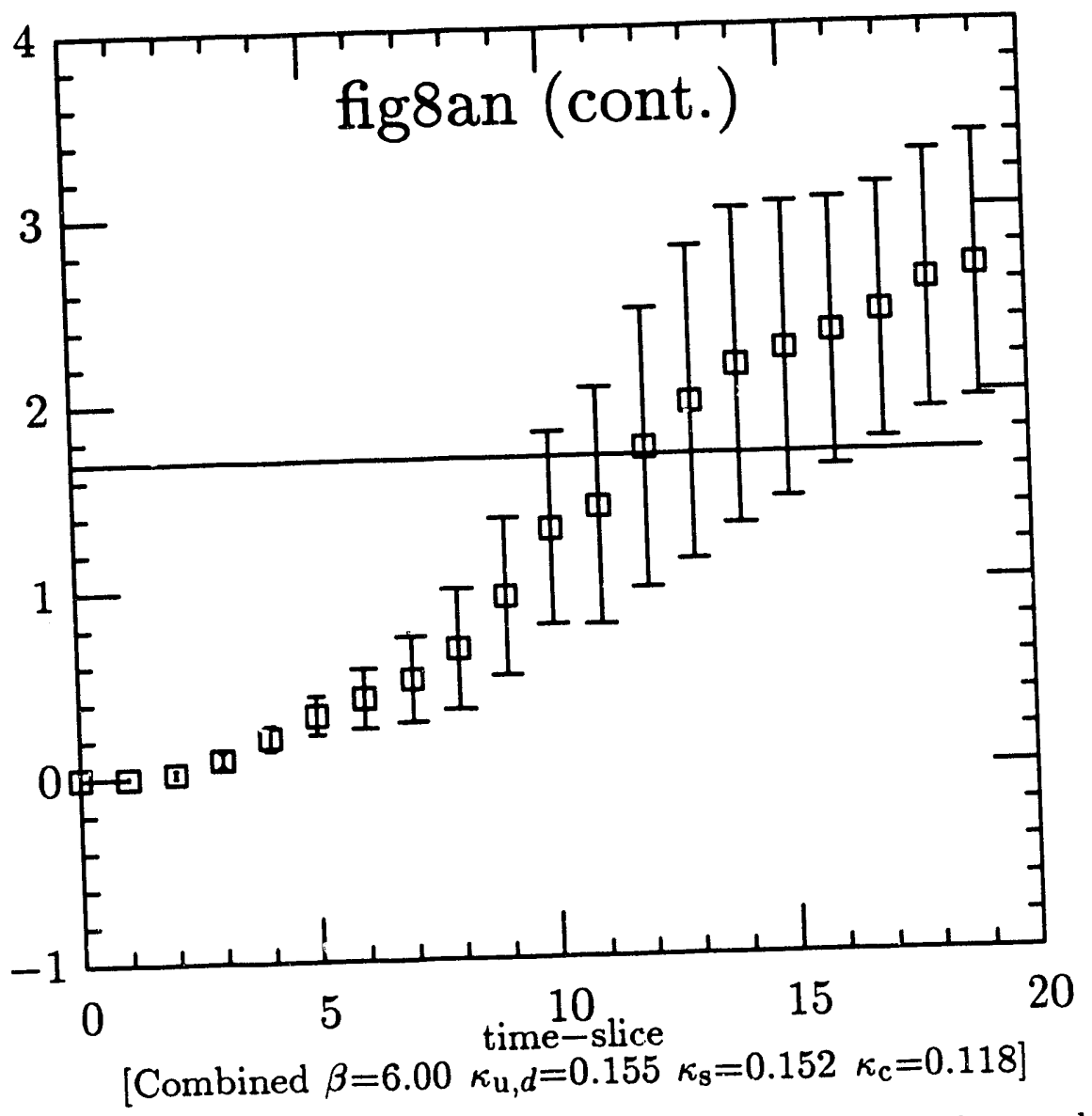

Figure 1.8.1: Fit to TI ratio for fig $8 \mathrm{an}^{\text {cont }}$ versus $t_{K}$ for the combined $\beta=6.0$ lattices. 


\section{I.9. $\mathcal{A}($ fig8K) $/ \mathcal{A}($ fig8 $\pi)$}

\begin{tabular}{|c|c|c|c|c|c|}
\hline $\begin{array}{l}\text { Lattice } \\
\text { (configs) }\end{array}$ & $\begin{array}{l}\text { Model } \\
\text { t Range }\end{array}$ & $\begin{array}{l}a_{1} \\
a_{2} \\
a_{3}\end{array}$ & Covar. & Coeff. & $\frac{\chi^{2}}{n_{d}}$ \\
\hline \multicolumn{6}{|c|}{$\kappa_{u, d}=.155, \kappa_{s}=.152, \kappa_{c}=.118$} \\
\hline $\begin{array}{l}\text { Combined } \\
\quad(27)\end{array}$ & $\begin{array}{c}I \\
{[12,16]}\end{array}$ & $\begin{array}{c}-0.35(7) \\
0.0^{*} \\
0.0^{*} \\
\end{array}$ & $\begin{array}{ll}1 & 0 \\
0 & 0 \\
0 & 0 \\
\end{array}$ & $\begin{array}{l}0 \\
0 \\
0 \\
0\end{array}$ & $\frac{3.6}{4}$ \\
\hline \multicolumn{6}{|c|}{$\kappa_{u, d}=.154, \kappa_{s}=.152, \kappa_{c}=.118$} \\
\hline $\begin{array}{l}\text { Combined } \\
\quad(27)\end{array}$ & $\begin{array}{c}I \\
{[12,16]}\end{array}$ & $\begin{array}{c}-0.32(6) \\
0.0^{*} \\
0.0^{*}\end{array}$ & $\begin{array}{ll}1 & 0 \\
0 & 0 \\
0 & 0 \\
\end{array}$ & $\begin{array}{ll}0 & 0 \\
0 & 0 \\
0 & 0\end{array}$ & $\frac{1.7}{4}$ \\
\hline \multicolumn{6}{|c|}{$\kappa_{u, d}=.152, \kappa_{s}=.152, \kappa_{c}=.118$} \\
\hline $\begin{array}{l}\text { Combined } \\
\quad(27)\end{array}$ & $\begin{array}{c}I \\
{[12,16]}\end{array}$ & $\begin{array}{c}-0.25(4) \\
0.0^{*} \\
0.0^{*}\end{array}$ & $\begin{array}{ll}1 & 0 \\
0 & 0 \\
0 & 0\end{array}$ & $\begin{array}{ll}0 & 0 \\
0 & 0 \\
0 & 0 \\
\end{array}$ & $\frac{0.92}{4}$ \\
\hline
\end{tabular}

Table 1.9.1: Fits to fig $8 \mathrm{~K} /$ fig $8 \pi$ versus $t_{K}$ for the $\beta=6.0$ lattices. Model Function I is $f(t)=a_{1}+a_{2} t+a_{3} t^{2}$. Model Function II is $f(t)=a_{1}+a_{2} e^{a_{3} t}$. Parameters that are held fixed are followed by an asterisk. 


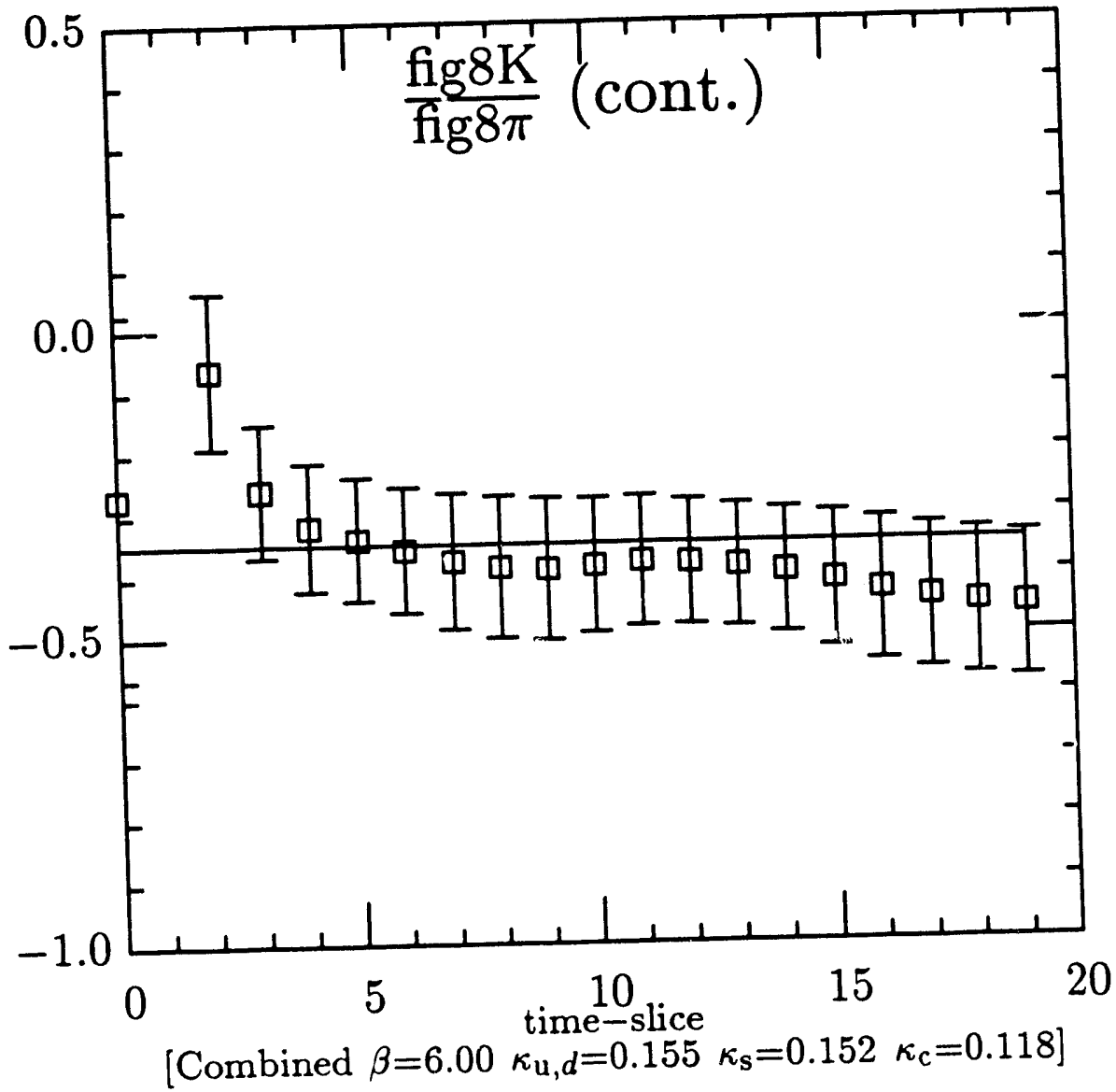

Figure 1.9.1: Fit to fig8K/fig8 8 versus $t_{K}$ for the combined $\beta=6.0$ lattices. 
I.10. $\mathcal{A}\left(\mathrm{fig} 8 \mathrm{~K}^{V S}\right) / \mathcal{A}\left(\mathrm{fig} 8 \pi^{V S}\right)$

\begin{tabular}{|c|c|c|c|c|}
\hline $\begin{array}{l}\text { Lattice } \\
\text { (configs) }\end{array}$ & $\begin{array}{l}\text { Model } \\
\text { t Range }\end{array}$ & $\begin{array}{l}a_{1} \\
a_{2} \\
a_{3} \\
\end{array}$ & Covar. Coeff. & $\frac{\chi^{2}}{n_{d}}$ \\
\hline \multicolumn{5}{|c|}{$\kappa_{u, d}=.155, \kappa_{s}=.152, \kappa_{c}=.118$} \\
\hline $\begin{array}{l}\text { Combined } \\
\quad(27)\end{array}$ & $\begin{array}{c}I \\
{[12,16]}\end{array}$ & $\begin{array}{c}0.15(1) \\
0.0^{*} \\
0.0^{*} \\
\end{array}$ & $\begin{array}{lll}1 & 0 & 0 \\
0 & 0 & 0 \\
0 & 0 & 0 \\
\end{array}$ & $\frac{2.2}{4}$ \\
\hline \multicolumn{5}{|c|}{$\kappa_{u, d}=.154, \kappa_{s}=.152, \kappa_{c}=.118$} \\
\hline $\begin{array}{l}\text { Combined } \\
\quad(27)\end{array}$ & $\begin{array}{c}I \\
{[12,16]}\end{array}$ & $\begin{array}{c}0.12(1) \\
0.0^{*} \\
0.0^{*} \\
\end{array}$ & $\begin{array}{lll}1 & 0 & 0 \\
0 & 0 & 0 \\
0 & 0 & 0 \\
\end{array}$ & $\frac{1.6}{4}$ \\
\hline \multicolumn{5}{|c|}{$\kappa_{u, d}=.152, \kappa_{s}=.152, \kappa_{c}=.118$} \\
\hline $\begin{array}{l}\text { Combined } \\
\quad(27)\end{array}$ & $\begin{array}{c}I \\
{[12,16]}\end{array}$ & $\begin{array}{c}0.098(4) \\
0.0^{*} \\
0.0^{*}\end{array}$ & $\begin{array}{lll}1 & 0 & 0 \\
0 & 0 & C \\
0 & 0 & 0\end{array}$ & $\frac{0.93}{4}$ \\
\hline
\end{tabular}

Table I.10.1: Fits to fig8 $8 \mathrm{~K}^{V S} /$ fig $8 \pi^{v S}$ versus $t_{K}$ for the $\beta=6.0$ lattices. Model Function I is $f(t)=a_{1}+a_{2} t+a_{3} t^{2}$. Model Function II is $f(t)=$ $a_{1}+a_{2} e^{a_{3} t}$. Parameters that are held fixed are followed by an asterisk. 


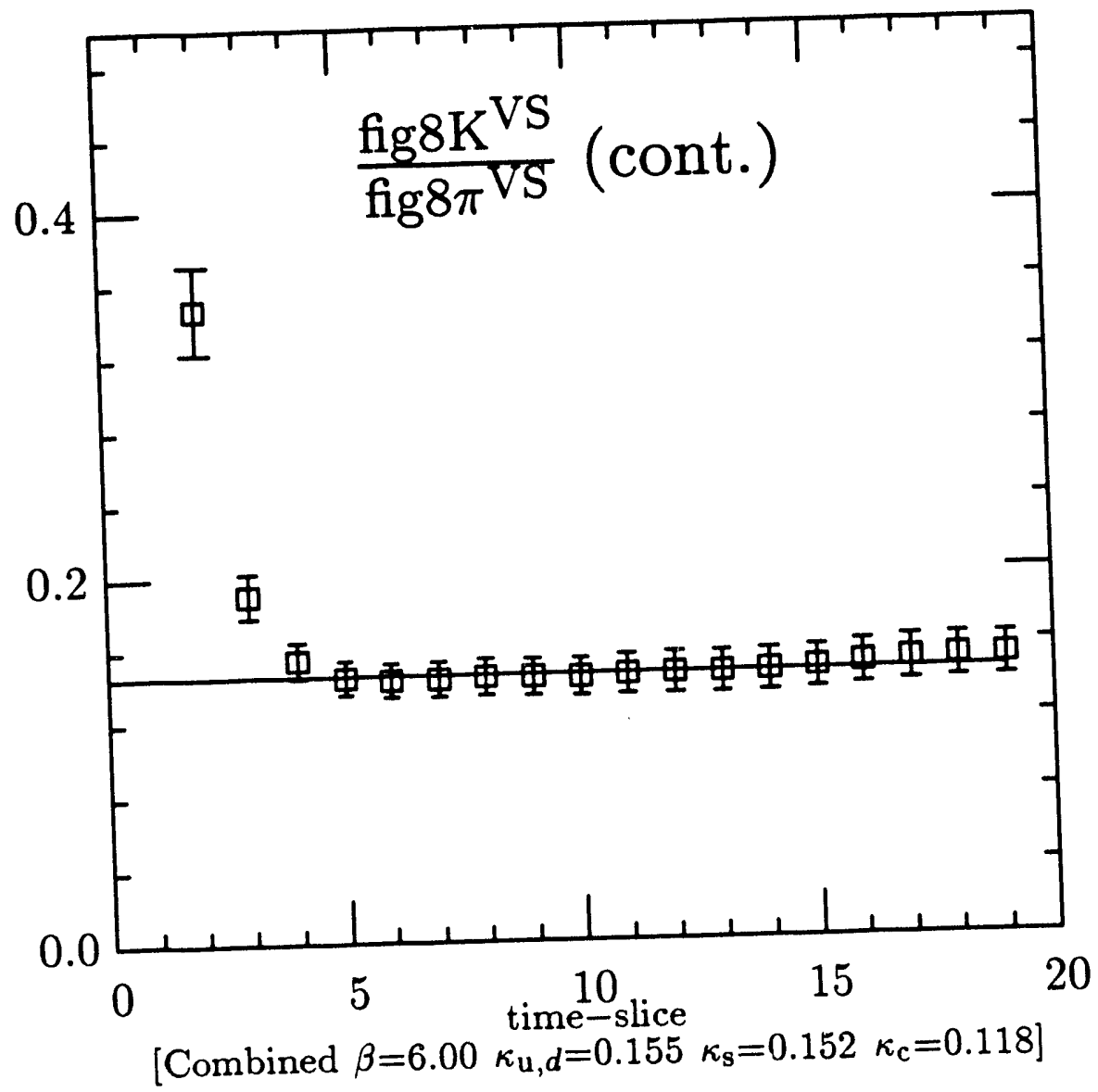

Figure 1.10.1: Fit to fig8 $\mathrm{K}^{V s} / \mathrm{fig} 8 \pi^{V s}$ versus $t_{K}$ for the combined $\beta=6.0$ lattices. 


\section{I.11. $\mathcal{A}($ flg8an $) / \mathcal{A}($ fig8 $\pi)$}

\begin{tabular}{|c|c|c|c|c|c|}
\hline $\begin{array}{l}\text { Lattice } \\
\text { (configs) }\end{array}$ & $\begin{array}{l}\text { Model } \\
\text { t Range }\end{array}$ & $\begin{array}{l}a_{1} \\
a_{2} \\
a_{3} \\
\end{array}$ & Covar. & Coeff. & $\frac{\chi^{2}}{n_{d}}$ \\
\hline \multicolumn{6}{|c|}{$\kappa_{u, d}=.155, \kappa_{s}=.152, \kappa_{c}=.118$} \\
\hline $\begin{array}{l}\text { Combined } \\
\quad(27)\end{array}$ & $\begin{array}{c}I \\
{[12,16]}\end{array}$ & $\begin{array}{c}1.1(5) \\
0.0^{*} \\
0.0^{*} \\
\end{array}$ & $\begin{array}{ll}1 & 0 \\
0 & 0 \\
0 & 0 \\
\end{array}$ & $\begin{array}{l}0 \\
0 \\
0 \\
\end{array}$ & $\frac{8.2}{4}$ \\
\hline \multicolumn{6}{|c|}{$\kappa_{u, d}=.154, \kappa_{s}=.152, \kappa_{c}=.118$} \\
\hline $\begin{array}{l}\text { Combined } \\
(27)\end{array}$ & $\begin{array}{c}I \\
{[12,16]}\end{array}$ & $\begin{array}{c}0.84(42) \\
0.0^{*} \\
0.0^{*} \\
\end{array}$ & $\begin{array}{ll}1 & 0 \\
0 & 0 \\
0 & 0 \\
\end{array}$ & $\begin{array}{l}0 \\
0 \\
0 \\
\end{array}$ & $\frac{5.1}{4}$ \\
\hline \multicolumn{6}{|c|}{$\kappa_{u, d}=.152, \kappa_{s}=.152, \kappa_{c}=.118$} \\
\hline $\begin{array}{l}\text { Combined } \\
(27)\end{array}$ & $\begin{array}{c}I \\
{[12,16]}\end{array}$ & $\begin{array}{c}0.76(43) \\
0.0^{*} \\
0.0^{*}\end{array}$ & $\begin{array}{ll}1 & 0 \\
0 & 0 \\
0 & 0\end{array}$ & $\begin{array}{ll}0 & 0 \\
0 & 0 \\
0 & 0\end{array}$ & $\frac{3.3}{4}$ \\
\hline
\end{tabular}

Table I.11.1: Fits to fig8an/fig8 8 versus $t_{K}$ for the $\beta=6.0$ lattices. Model Function I is $f(t)=a_{1}+a_{2} t+a_{3} t^{2}$. Model Function II is $f(t)=a_{1}+a_{2} e^{a_{3} t}$. Parameters that are held fixed are followed by an asterisk. 


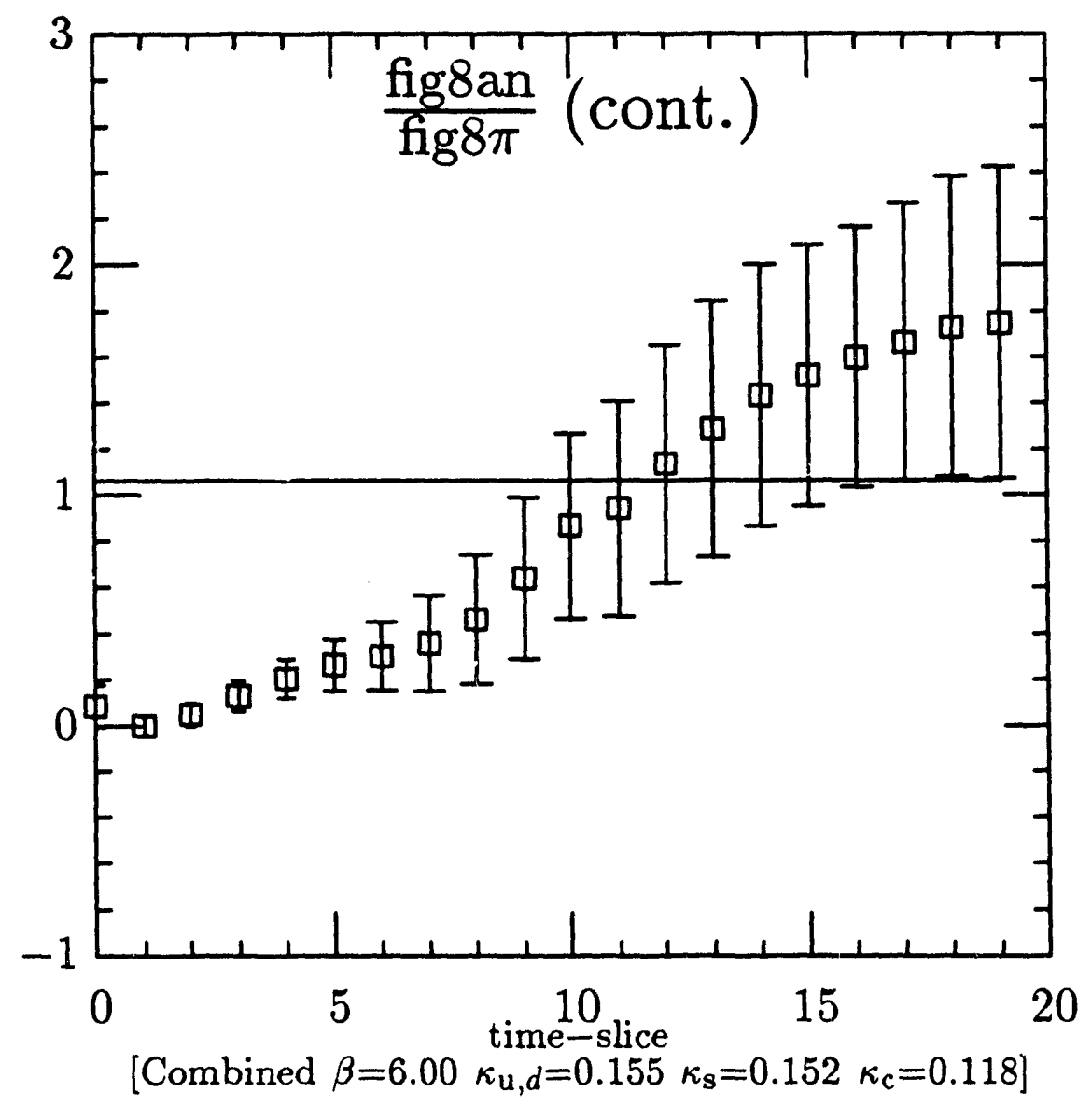

Figure 1.11.1: Fit to fig8an $/$ fig $8 \pi$ versus $t_{K}$ for the combined $\beta=6.0$ lattices. 


\section{I.12. $\mathcal{A}\left(\mathrm{flg}_{8 \mathrm{an}}{ }^{V S}\right) / \mathcal{A}\left(\mathrm{fig} 8 \boldsymbol{\pi}^{V S}\right)$}

\begin{tabular}{|c|c|c|c|c|}
\hline $\begin{array}{l}\text { Lattice } \\
\text { (configs) }\end{array}$ & $\begin{array}{l}\text { Model } \\
\text { t Range }\end{array}$ & $\begin{array}{l}a_{1} \\
a_{2} \\
a_{3} \\
\end{array}$ & Covar. Coeff. & $\frac{\chi^{2}}{n_{d}}$ \\
\hline \multicolumn{5}{|c|}{$\kappa_{u, d}=.155, \kappa_{s}=.152, \kappa_{c}=.118$} \\
\hline $\begin{array}{l}\text { Combined } \\
(27)\end{array}$ & $\begin{array}{c}I \\
{[12,16]}\end{array}$ & $\begin{array}{c}-0.19(3) \\
0.0^{*} \\
0.0^{*} \\
\end{array}$ & $\begin{array}{lll}1 & 0 & 0 \\
0 & 0 & 0 \\
0 & 0 & 0 \\
\end{array}$ & $\frac{7.9}{4}$ \\
\hline \multicolumn{5}{|c|}{$\kappa_{u, d}=.154, \kappa_{s}=.152, \kappa_{c}=.118$} \\
\hline $\begin{array}{l}\text { Combined } \\
\quad(27)\end{array}$ & $\begin{array}{c}I \\
{[12,16]}\end{array}$ & $\begin{array}{c}-0.14(2) \\
0.0^{*} \\
0.0^{*}\end{array}$ & $\begin{array}{lll}1 & 0 & 0 \\
0 & 0 & 0 \\
0 & 0 & 0 \\
\end{array}$ & $\frac{5.3}{4}$ \\
\hline \multicolumn{5}{|c|}{$\kappa_{u, d}=.152, \kappa_{s}=.152, \kappa_{c}=.118$} \\
\hline $\begin{array}{l}\text { Combined } \\
\quad(27)\end{array}$ & $\begin{array}{c}I \\
{[12,16]}\end{array}$ & $\begin{array}{c}-0.018(23) \\
0.0^{*} \\
0.0^{*}\end{array}$ & $\begin{array}{lll}1 & 0 & 0 \\
0 & 0 & 0 \\
0 & 0 & 0\end{array}$ & $\frac{2.1}{4}$ \\
\hline
\end{tabular}

Table 1.12.1: Fits to fig8an ${ }^{V S} /$ fig $8 \pi^{V S}$ versus $t_{K}$ for the $\beta=6.0$ lattices. Model Function I is $f(t)=a_{1}+a_{2} t+a_{3} t^{2}$. Model Function II is $f(t)=$ $a_{1}+a_{2} e^{a_{3} t}$. Parameters that are held fixed are followed by an asterisk. 


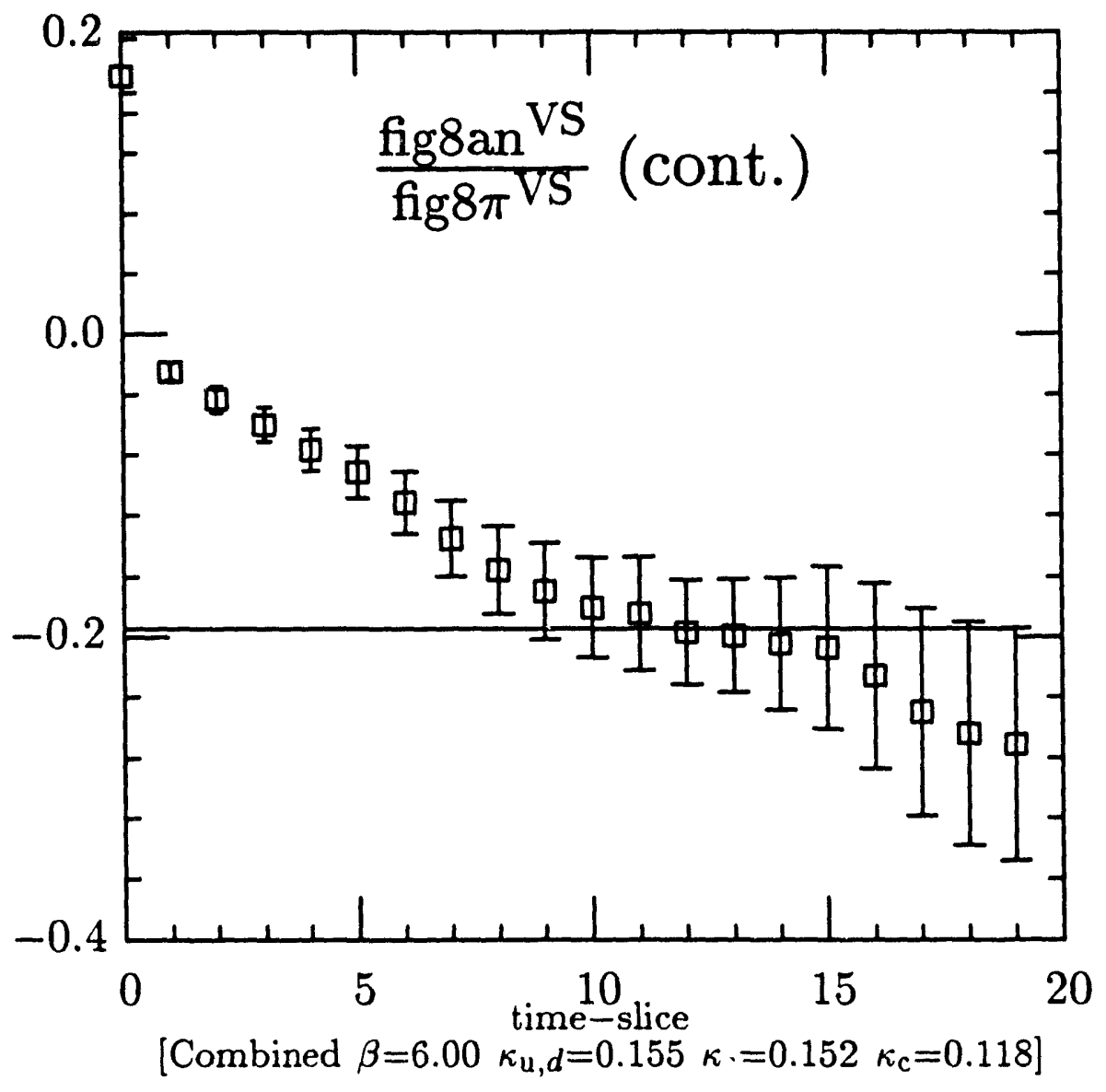

Figure I.12.1: Fit to fig8an ${ }^{V S} /$ fig $8 \pi^{V S}$ versus $t_{K}$ for the combined $\beta=6.0$ lattices. 
I.13. $\mathcal{A}_{3 / 2}$

\begin{tabular}{|c|c|c|c|c|}
\hline $\begin{array}{l}\text { Lattice } \\
\text { (configs) }\end{array}$ & $\begin{array}{l}\text { Model } \\
\text { t Range }\end{array}$ & $\begin{array}{l}a_{1} \\
a_{2} \\
a_{3} \\
\end{array}$ & Covar. Coeff. & $\frac{\chi^{2}}{n_{d}}$ \\
\hline \multicolumn{5}{|c|}{$\kappa_{u, d}=.155, \kappa_{s}=.152, \kappa_{c}=.118$} \\
\hline $\begin{array}{l}\text { Combined } \\
\quad(27)\end{array}$ & $\begin{array}{c}I \\
{[12,16]}\end{array}$ & $\begin{array}{c}0.55(6) \\
0.0^{*} \\
0.0^{*} \\
\end{array}$ & $\begin{array}{lll}1 & 0 & 0 \\
0 & 0 & 0 \\
0 & 0 & 0 \\
\end{array}$ & $\frac{5.2}{4}$ \\
\hline \multicolumn{5}{|c|}{$\kappa_{u, d}=.154, \kappa_{s}=.152, \kappa_{c}=.118$} \\
\hline $\begin{array}{l}\text { Combined } \\
\quad(27)\end{array}$ & $\begin{array}{c}I \\
{[12,16]}\end{array}$ & $\begin{array}{c}0.60(5) \\
0.0^{*} \\
0.0^{*} \\
\end{array}$ & $\begin{array}{lll}1 & 0 & 0 \\
0 & 0 & 0 \\
0 & 0 & 0 \\
\end{array}$ & $\frac{2.2}{4}$ \\
\hline \multicolumn{5}{|c|}{$\kappa_{u, d}=.152, \kappa_{s}=.152, \kappa_{c}=.118$} \\
\hline $\begin{array}{l}\text { Combined } \\
\quad(27)\end{array}$ & $\begin{array}{c}I \\
{[12,16]}\end{array}$ & $\begin{array}{c}0.66(6) \\
0.0^{*} \\
0.0^{*}\end{array}$ & $\begin{array}{lll}1 & 0 & 0 \\
0 & 0 & 0 \\
0 & 0 & 0\end{array}$ & $\frac{3.6}{4}$ \\
\hline
\end{tabular}

Table I.13.1: Fits to TI ratio for $G_{3 / 2}^{(4)}$ versus $t_{K}$ for the $\beta=6.0$ lattices. Model Function I is $f(t)=a_{1}+a_{2} t+a_{3} t^{2}$. Model Function II is $f(t)=$ $a_{1}+a_{2} e^{a_{s} t}$. Parameters that are held fixed are followed by an asterisk. 


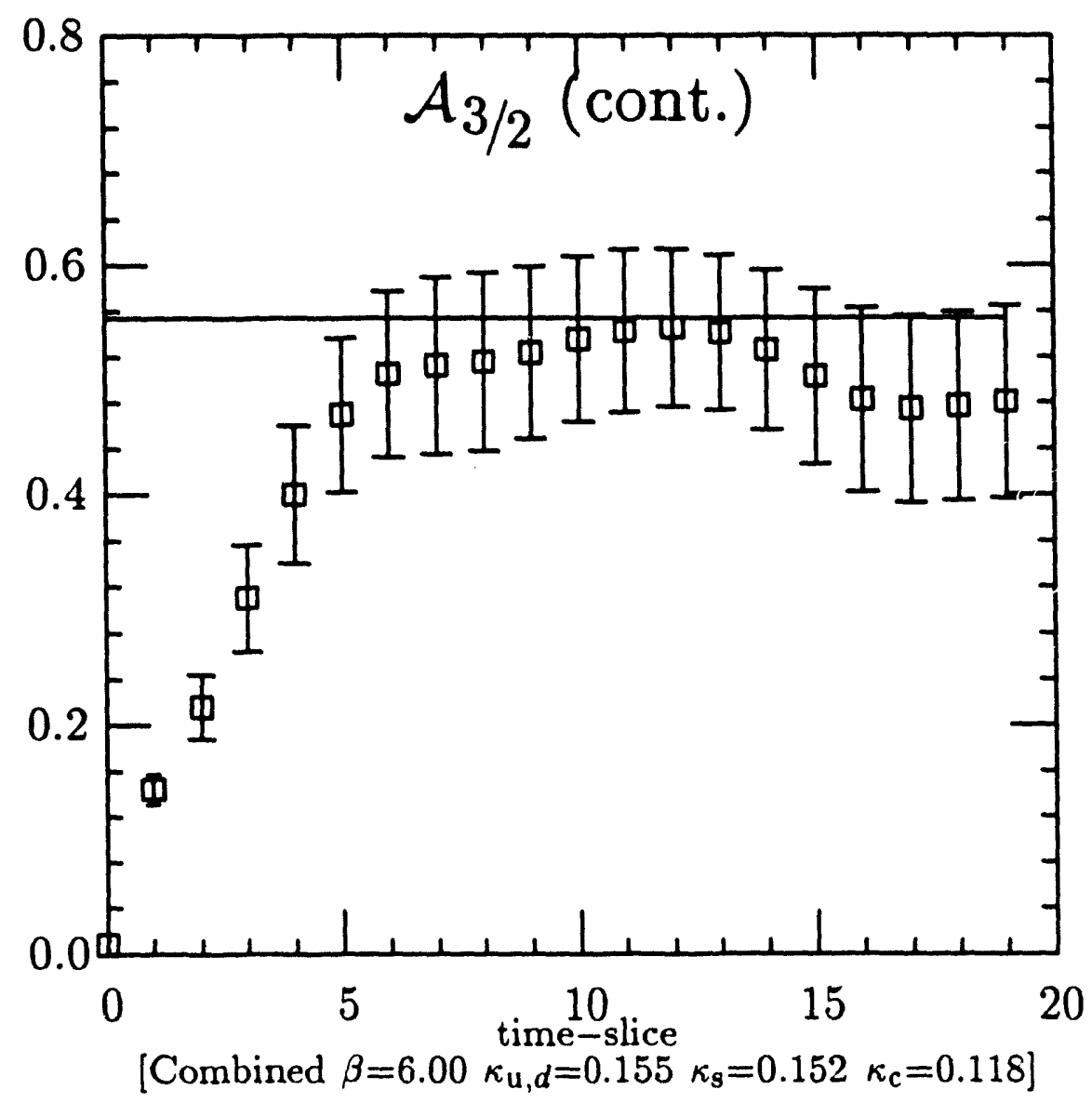

Figure I.13.1: Fit to TI ratio for $G_{3 / 2}^{(4)}$ versus $t_{K}$ for the combined $\beta=6.0$ lattices. 


\section{I.14. $\mathcal{A}_{3 / 2}^{V S}$}

\begin{tabular}{|c|c|c|c|c|}
\hline $\begin{array}{l}\text { Lattice } \\
\text { (configs) }\end{array}$ & $\begin{array}{l}\text { Model } \\
\text { t Range }\end{array}$ & $\begin{array}{l}a_{1} \\
a_{2} \\
a_{3}\end{array}$ & Covar. Coeff. & $\frac{\chi^{2}}{n_{d}}$ \\
\hline \multicolumn{5}{|c|}{$\kappa_{u, d}=.155, \kappa_{\imath}=.152, \kappa_{c}=.118$} \\
\hline $\begin{array}{c}\text { Combined } \\
(27)\end{array}$ & $\begin{array}{c}I \\
{[12,16]}\end{array}$ & $\begin{array}{c}0.67(7) \\
0.0^{*} \\
0.0^{*}\end{array}$ & $\begin{array}{lll}1 & 0 & 0 \\
0 & 0 & 0 \\
0 & 0 & 0\end{array}$ & $\frac{2.5}{4}$ \\
\hline \multicolumn{5}{|c|}{$\kappa_{u, d}=.154, \kappa_{\imath}=.152, \kappa_{c}=.118$} \\
\hline $\begin{array}{c}\text { Combined } \\
\text { (27) }\end{array}$ & $\begin{array}{c}I \\
{[12,16]}\end{array}$ & $\begin{array}{c}0.73(7) \\
0.0^{\circ} \\
0.0^{*}\end{array}$ & $\begin{array}{lll}1 & 0 & 0 \\
0 & 0 & 0 \\
0 & 0 & 0\end{array}$ & $\frac{2.6}{4}$ \\
\hline \multicolumn{5}{|c|}{$\kappa_{u, d}=.152, \kappa_{,}=.152, \kappa_{c}=.118$} \\
\hline $\begin{array}{c}\text { Combined } \\
(27)\end{array}$ & $\begin{array}{c}I \\
{[12,16]}\end{array}$ & $\begin{array}{c}0.80(6) \\
0.0^{*} \\
0.0^{*}\end{array}$ & $\begin{array}{lll}1 & 0 & 0 \\
0 & 0 & 0 \\
0 & 0 & 0 \\
\end{array}$ & $\frac{3.8}{4}$ \\
\hline
\end{tabular}

Table I.14.1: Fits to TI ratio $G_{3 / 2}{ }^{v S}$ versus $t_{K}$ for the $\beta=6.0$ lattices. Model Funciion I is $f(t)=a_{1}+a_{2} t+a_{3} t^{2}$. Model Function II is $f(t)=$ $a_{1}+a_{2} e^{a_{3} t}$. Parameters that are held fixed are followed by an asterisk. 


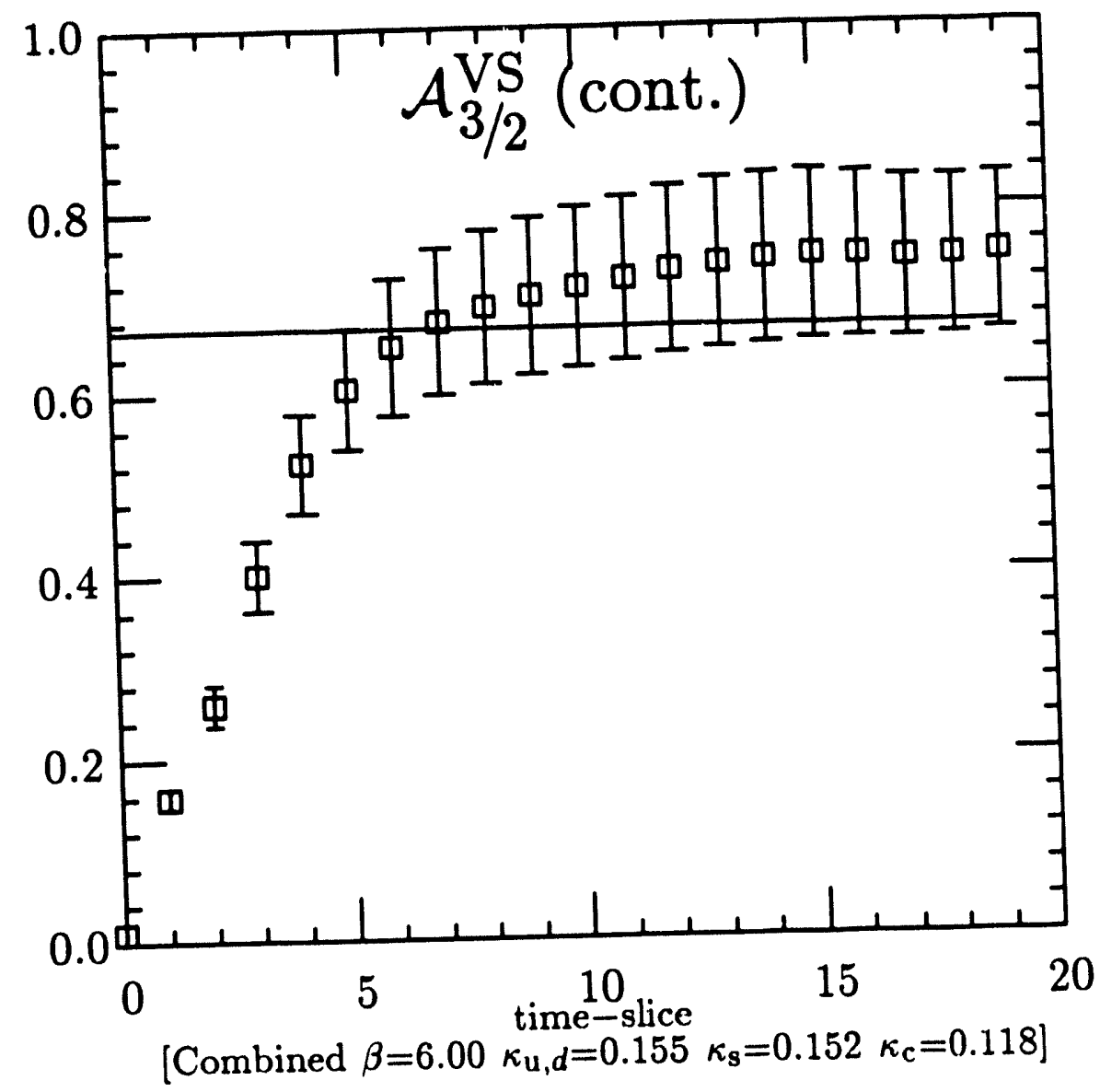

Figure 1.14.1: Fit to TI ratio for $G_{3 / 2}{ }^{v s}$ for the combined $\beta=6.0$ lattices. 
I.15. $\mathcal{A}_{3 / 2} / \mathcal{A}_{3 / 2}^{V S}$

\begin{tabular}{|c|c|c|c|c|}
\hline $\begin{array}{l}\text { Lattice } \\
\text { (configs) }\end{array}$ & $\begin{array}{l}\text { Model } \\
\text { t Range }\end{array}$ & $\begin{array}{l}a_{1} \\
a_{2} \\
a_{3}\end{array}$ & Covar. Coeff. & $\frac{\chi^{2}}{n_{d}}$ \\
\hline \multicolumn{5}{|c|}{$\kappa_{u, d}=.155, \kappa_{s}=.152, \kappa_{c}=.118$} \\
\hline $\begin{array}{l}\text { Combined } \\
(27)\end{array}$ & $\begin{array}{c}I \\
{[12,16]}\end{array}$ & $\begin{array}{c}0.74(4) \\
0.0^{\circ} \\
0.0^{*}\end{array}$ & $\begin{array}{lll}1 & 0 & 0 \\
0 & 0 & 0 \\
0 & 0 & 0 \\
\end{array}$ & $\frac{5.4}{4}$ \\
\hline \multicolumn{5}{|c|}{$\kappa_{u, d}=.154, \kappa_{s}=.152, \kappa_{c}=.118$} \\
\hline $\begin{array}{c}\text { Combined } \\
(27)\end{array}$ & $\begin{array}{c}I \\
{[12,16]}\end{array}$ & $\begin{array}{c}0.78(3) \\
0.0^{*} \\
0.0^{*}\end{array}$ & $\begin{array}{lll}1 & 0 & 0 \\
0 & 0 & 0 \\
0 & 0 & 0 \\
\end{array}$ & $\frac{4.4}{4}$ \\
\hline \multicolumn{5}{|c|}{$\kappa_{u, d}=.152, \kappa_{\imath}=.152, \kappa_{c}=.118$} \\
\hline $\begin{array}{c}\text { Combined } \\
(27)\end{array}$ & $\begin{array}{c}I \\
{[12,16]}\end{array}$ & $\begin{array}{c}0.84(2) \\
0.0^{*} \\
0.0^{*}\end{array}$ & $\begin{array}{lll}1 & 0 & 0 \\
0 & 0 & 0 \\
0 & 0 & 0\end{array}$ & $\frac{4.8}{4}$ \\
\hline
\end{tabular}

Table 1.15.1: Fits to $G_{3 / 2}^{(4)} / G_{3 / 2} v s$ versus $t_{K}$ for the $\beta=6.0$ lattices. Model Function I is $f(t)=a_{1}+a_{2} t+a_{3} t^{2}$. Model Function II is $f(t)=a_{1}+a_{2} e^{a_{3} t}$. Parameters that are held fixed are followed by an asterisk. 


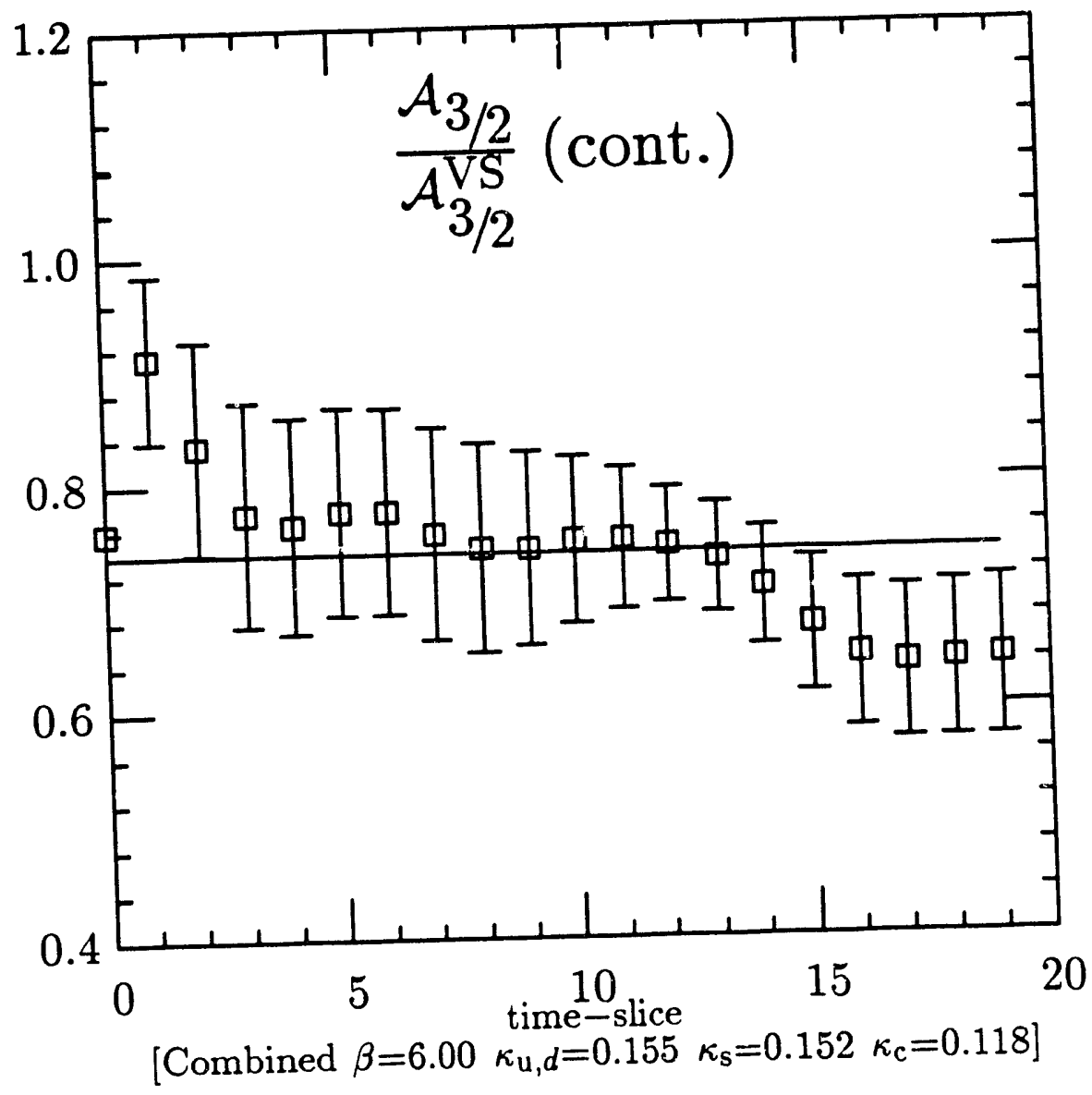

Figure 1.15.1: Fit to $G_{3 / 2}^{(4)} / G_{3 / 2} v S$ versus $t_{K}$ for the combined $\beta=6.0$ lattices. 


\section{I.16. $\mathcal{A}_{1 / 2}$}

\begin{tabular}{|c|c|c|c|c|}
\hline $\begin{array}{l}\text { Lattice } \\
\text { (configs) }\end{array}$ & $\begin{array}{l}\text { Model } \\
\text { t Range }\end{array}$ & $\begin{array}{l}a_{1} \\
a_{2} \\
a_{3}\end{array}$ & Covar. Coeff. & $\frac{\chi^{2}}{n_{d}}$ \\
\hline \multicolumn{5}{|c|}{$\kappa_{u, d}=.155, \kappa_{s}=.152, \kappa_{e}=.118$} \\
\hline $\begin{array}{l}\text { Combined } \\
\quad(27)\end{array}$ & $\begin{array}{c}I \\
{[12,16]}\end{array}$ & $\begin{array}{c}3.4(7) \\
0.0^{*} \\
0.0^{*}\end{array}$ & $\begin{array}{lll}1 & 0 & 0 \\
0 & 0 & 0 \\
0 & 0 & 0 \\
\end{array}$ & $-\frac{6.5}{4}$ \\
\hline \multicolumn{5}{|c|}{$\kappa_{u, d}=.154, \kappa_{s}=.152, \kappa_{c}=.118$} \\
\hline $\begin{array}{c}\text { Combined } \\
(27)\end{array}$ & $\begin{array}{c}I \\
{[12,16]}\end{array}$ & $\begin{array}{c}3.3(6) \\
0.0^{*} \\
0.0^{*} \\
\end{array}$ & $\begin{array}{lll}1 & 0 & 0 \\
0 & 0 & 0 \\
0 & 0 & 0 \\
\end{array}$ & $\frac{5.4}{4}$ \\
\hline \multicolumn{5}{|c|}{$\kappa_{u, d}=.152, \kappa_{s}=.152, \kappa_{c}=.118$} \\
\hline $\begin{array}{c}\text { Combined } \\
(27)\end{array}$ & $\begin{array}{c}I \\
{[12,16]}\end{array}$ & $\begin{array}{c}3.2(8) \\
0.0^{*} \\
0.0^{*}\end{array}$ & $\begin{array}{lll}1 & 0 & 0 \\
0 & 0 & 0 \\
0 & 0 & 0\end{array}$ & $\frac{4.1}{4}$ \\
\hline
\end{tabular}

Table I.16.1: Fits to TI ratio for $G_{1 / 2}^{(4)}$ versus $t_{K}$ for the $\beta=6.0$ lattices. Model Function I is $f(t)=a_{1}+a_{2} t+a_{3} t^{2}$. Model Function II is $f(t)=$ $a_{1}+a_{2} e^{a_{3} t}$. Parameters that are held fixed are followed by an asterisk. 


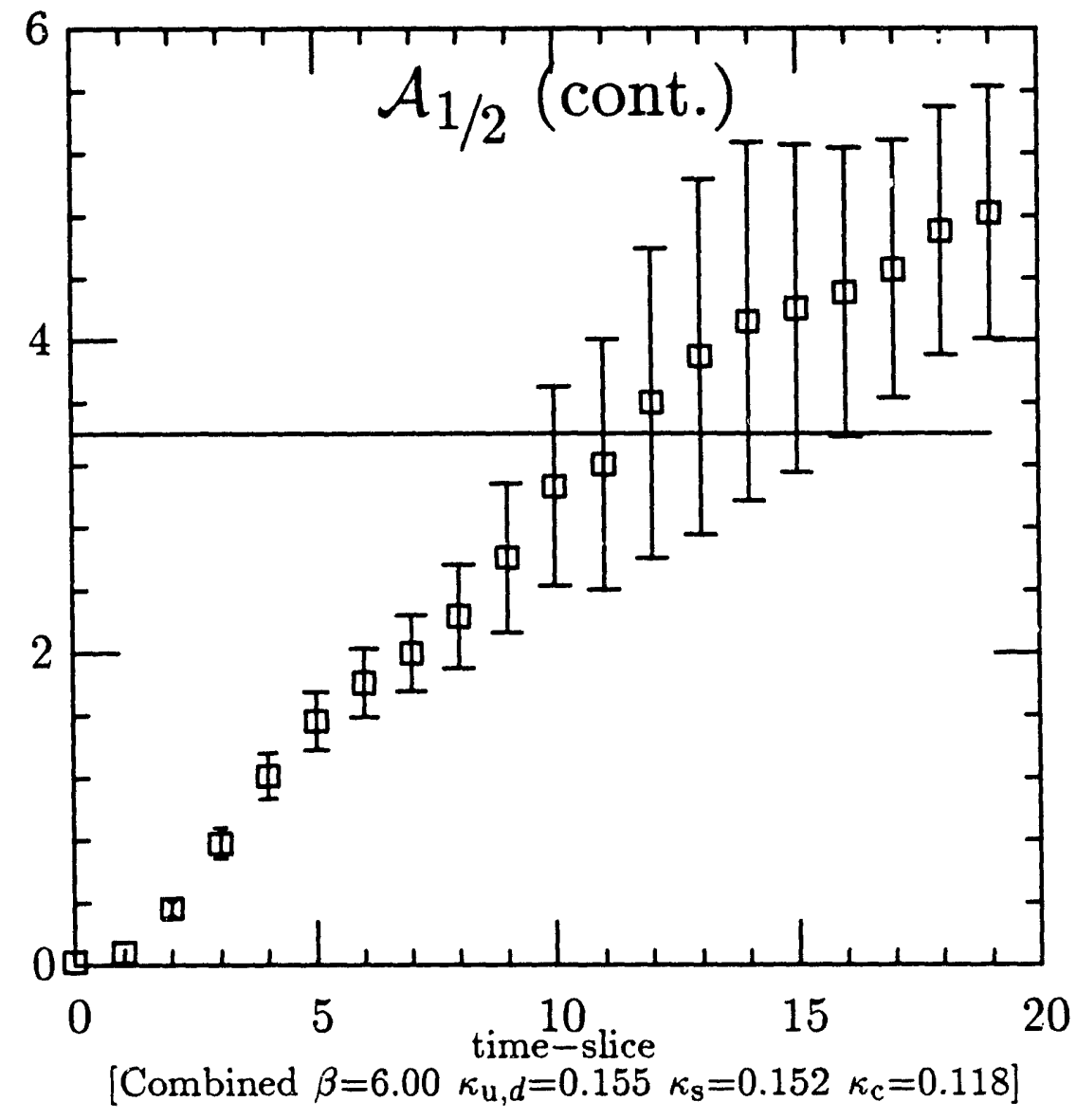

Figure 1.16.1: Fit to TI ratio for $G_{1 / 2}^{(4)}$ versus $t_{K}$ for the combined $\beta=6.0$ lattices. 
I.17. $\mathcal{A}_{1 / 2}^{V S}$

\begin{tabular}{|c|c|c|c|c|c|}
\hline $\begin{array}{l}\text { Lattice } \\
\text { (configs) }\end{array}$ & $\begin{array}{l}\text { Model } \\
\text { t Range }\end{array}$ & $\begin{array}{l}a_{1} \\
a_{2} \\
a_{3}\end{array}$ & Covar. & Coeff. & $\frac{\chi^{2}}{n_{d}}$ \\
\hline \multicolumn{6}{|c|}{$\kappa_{u, d}=.155, \kappa_{s}=.152, \kappa_{c}=.118$} \\
\hline $\begin{array}{l}\text { Combined } \\
\quad(27)\end{array}$ & $\begin{array}{c}I \\
{[12,16]}\end{array}$ & $\begin{array}{c}0.51(9) \\
0.0^{*} \\
0.0^{*}\end{array}$ & $\begin{array}{ll}1 & 0 \\
0 & 0 \\
0 & 0 \\
\end{array}$ & $\begin{array}{l}0 \\
0 \\
0\end{array}$ & $\frac{8.1}{4}$ \\
\hline \multicolumn{6}{|c|}{$\kappa_{u, d}=.154, \kappa_{s}=.152, \kappa_{c}=.118$} \\
\hline $\begin{array}{l}\text { Combined } \\
(27)\end{array}$ & $\begin{array}{c}I \\
{[12,16]}\end{array}$ & $\begin{array}{c}0.68(8) \\
0.0^{*} \\
0.0^{*}\end{array}$ & $\begin{array}{ll}1 & 0 \\
0 & 0 \\
0 & 0 \\
\end{array}$ & $\begin{array}{l}0 \\
0 \\
0\end{array}$ & $\frac{6.4}{4}$ \\
\hline \multicolumn{6}{|c|}{$\kappa_{u, d}=.152, \kappa_{s}=.152, \kappa_{c}=.118$} \\
\hline $\begin{array}{l}\text { Combined } \\
\quad(27)\end{array}$ & $\begin{array}{c}I \\
{[12,16]}\end{array}$ & $\begin{array}{c}0.97(6) \\
0.0^{*} \\
0.0^{*}\end{array}$ & $\begin{array}{ll}1 & 0 \\
0 & 0 \\
0 & 0 \\
\end{array}$ & $\begin{array}{l}0 \\
0 \\
0\end{array}$ & $\frac{2.5}{4}$ \\
\hline
\end{tabular}

Table 1.17.1: Fits to TI ratio for $G_{1 / 2}{ }^{V S}$ versus $t_{K}$ for the $\beta=6.0$ lattices. Model Function I is $f(t)=a_{1}+a_{2} t+a_{3} t^{2}$. Model Function II is $f(t)=$ $a_{1}+a_{2} e^{a_{3} t}$. Parameters that are held fixed are followed by an asterisk. 


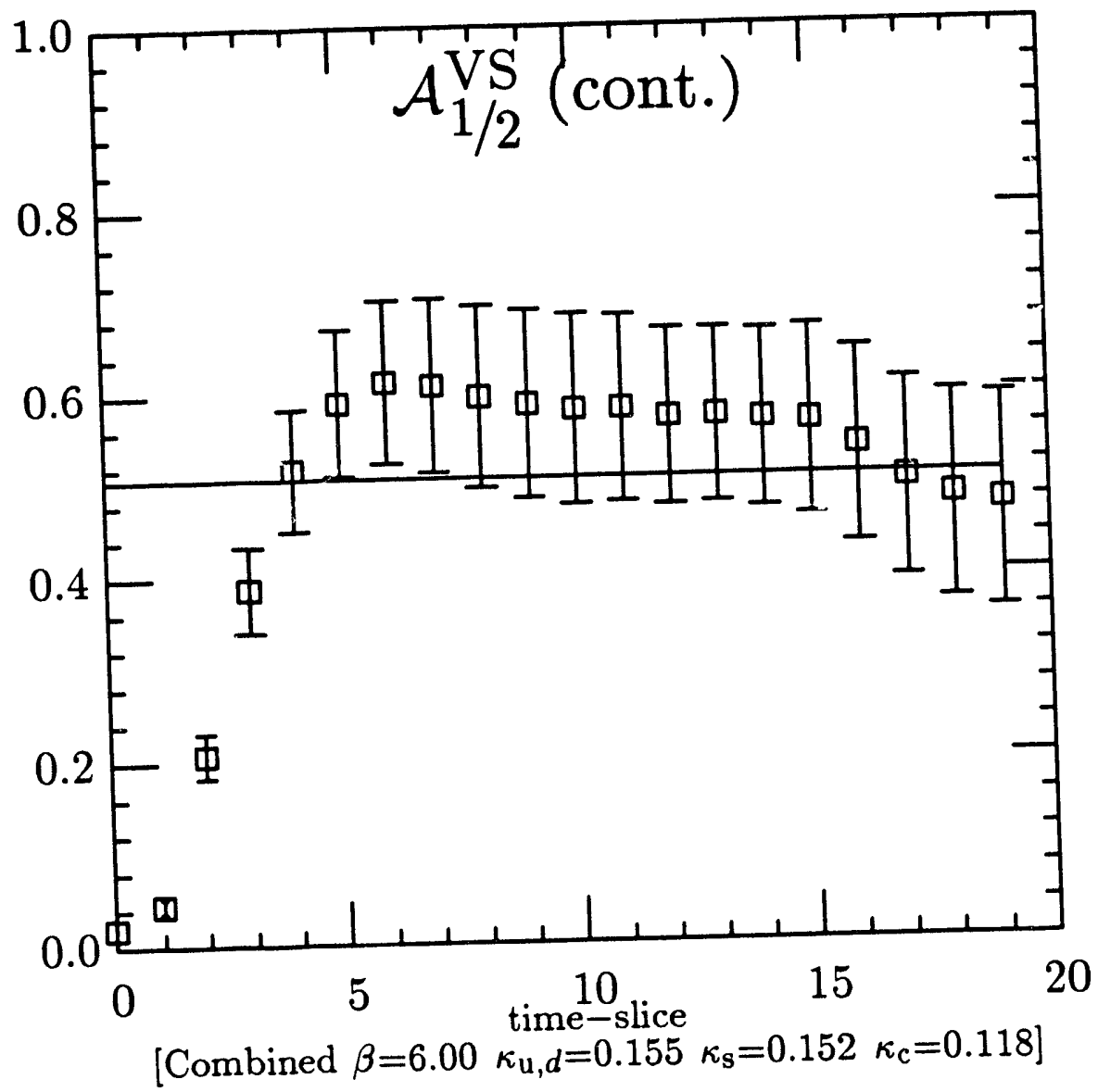

Figure I.17.1: Fit to TI ratio for $G_{1 / 2}{ }^{v S}$ versus $t_{K}$ for the combined $\beta=6.0$ lattices. 
I.18. $\mathcal{A}_{1 / 2} / \mathcal{A}_{1 / 2}^{V S}$

\begin{tabular}{|c|c|c|c|c|}
\hline $\begin{array}{l}\text { Lattice } \\
\text { (configs) }\end{array}$ & $\begin{array}{l}\text { Model } \\
\text { t Range }\end{array}$ & $\begin{array}{l}a_{1} \\
a_{2} \\
a_{3}\end{array}$ & Covar. Coeff. & $\frac{\chi^{2}}{n_{d}}$ \\
\hline \multicolumn{5}{|c|}{$\kappa_{u, d}=.155, \kappa_{s}=.152, \kappa_{c}=.118$} \\
\hline $\begin{array}{l}\text { Combined } \\
(27)\end{array}$ & $\begin{array}{c}I \\
{[12,16]}\end{array}$ & $\begin{array}{c}4.3(18) \\
0.0^{*} \\
0.0^{*} \\
\end{array}$ & $\begin{array}{lll}1 & 0 & 0 \\
0 & 0 & 0 \\
0 & 0 & 0 \\
\end{array}$ & $\frac{7.0}{4}$ \\
\hline \multicolumn{5}{|c|}{$\kappa_{u, d}=.154, \kappa_{s}=.152, \kappa_{c}=.118$} \\
\hline $\begin{array}{l}\text { Combined } \\
(27)\end{array}$ & $\begin{array}{c}I \\
{[12,16]}\end{array}$ & $\begin{array}{c}4.2(11) \\
0.0^{*} \\
0.0^{*}\end{array}$ & $\begin{array}{lll}1 & 0 & 0 \\
0 & 0 & 0 \\
0 & 0 & 0\end{array}$ & $\frac{7.2}{4}$ \\
\hline \multicolumn{5}{|c|}{$\kappa_{u, d}=.152, \kappa_{s}=.152, \kappa_{c}=.118$} \\
\hline $\begin{array}{l}\text { Combined } \\
\quad(27)\end{array}$ & $\begin{array}{c}I \\
{[12,16]}\end{array}$ & $\begin{array}{c}3.0(8) \\
0.0^{*} \\
0.0^{*}\end{array}$ & $\begin{array}{lll}1 & 0 & 0 \\
0 & 0 & 0 \\
0 & 0 & 0\end{array}$ & $\frac{3.8}{4}$ \\
\hline
\end{tabular}

Table I.18.1: Fits to $G_{1 / 2}^{(4)} / G_{1 / 2} v S$ versus $t_{K}$ for the $\beta=6.0$ lattices. Model Fun tion I is $f(t)=a_{1}+a_{2} t+a_{3} t^{2}$. Model Function II is $f(t)=a_{1}+a_{2} e^{a_{3} t}$. Parameters that are held fixed are followed by an asterisk. 


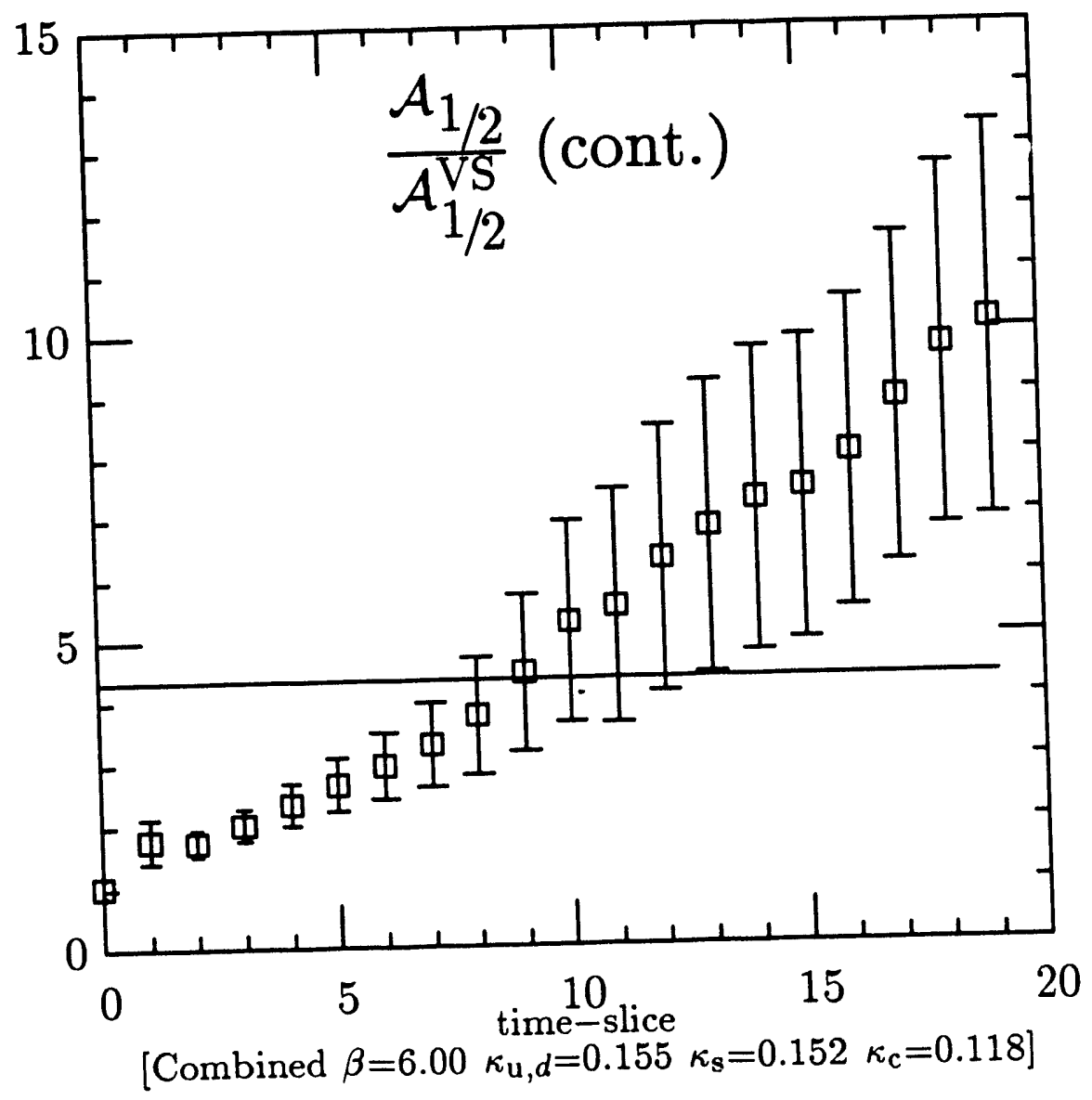

Figure I.18.1: Fit to $G_{1 / 2}^{(4)} / G_{1 / 2} v S$ versus $t_{K}$ for the combined $\beta=6.0$ lattices. 
I.19. $\mathcal{A}_{1 / 2} / \mathcal{A}_{3 / 2}$

\begin{tabular}{|c|c|c|c|c|}
\hline $\begin{array}{l}\text { Lattice } \\
\text { (configs) }\end{array}$ & $\begin{array}{l}\text { Model } \\
\text { t Range }\end{array}$ & $\begin{array}{l}a_{1} \\
a_{2} \\
a_{3} \\
\end{array}$ & Covar. Coeff. & $\frac{\chi^{2}}{n_{d}}$ \\
\hline \multicolumn{5}{|c|}{$\kappa_{u, d}=.155, \kappa_{s}=.152, \kappa_{c}=.118$} \\
\hline $\begin{array}{l}\text { Combined } \\
(27)\end{array}$ & $\begin{array}{c}I \\
{[12,16]}\end{array}$ & $\begin{array}{c}5.1(18) \\
0.0^{*} \\
0.0^{*} \\
\end{array}$ & $\begin{array}{lll}1 & 0 & 0 \\
0 & 0 & 0 \\
0 & 0 & 0 \\
\end{array}$ & $\frac{7.4}{4}$ \\
\hline \multicolumn{5}{|c|}{$\kappa_{u, d}=.154, \kappa_{s}=.152, \kappa_{c}=.118$} \\
\hline $\begin{array}{l}\text { Combined } \\
\quad(27)\end{array}$ & $\begin{array}{c}I \\
{[12,16]}\end{array}$ & $\begin{array}{c}5.2(14) \\
0.0^{*} \\
0.0^{*}\end{array}$ & $\begin{array}{lll}1 & 0 & 0 \\
0 & 0 & 0 \\
0 & 0 & 0 \\
\end{array}$ & $\frac{5.2}{4}$ \\
\hline \multicolumn{5}{|c|}{$\kappa_{u, d}=.152, \kappa_{s}=.152, \kappa_{c}=.118$} \\
\hline $\begin{array}{l}\text { Combined } \\
\quad(27)\end{array}$ & $\begin{array}{c}I \\
{[12,16]}\end{array}$ & $\begin{array}{c}4.2(12) \\
0.0^{*} \\
0.0^{*}\end{array}$ & $\begin{array}{lll}1 & 0 & 0 \\
0 & 0 & 0 \\
0 & 0 & 0\end{array}$ & $\frac{3.4}{4}$ \\
\hline
\end{tabular}

Table 1.19.1: Fits to $G_{1 / 2}^{(4)} / G_{3 / 2}^{(4)}$ versus $t_{K}$ for the $\beta=6.0$ lattices. Model Function I is $f(t)=a_{1}+a_{2} t+a_{3} t^{2}$. Model Function II is $f(t)=a_{1}+a_{2} e^{a_{3} t}$. Parameters that are held fixed are followed by an asterisk. 


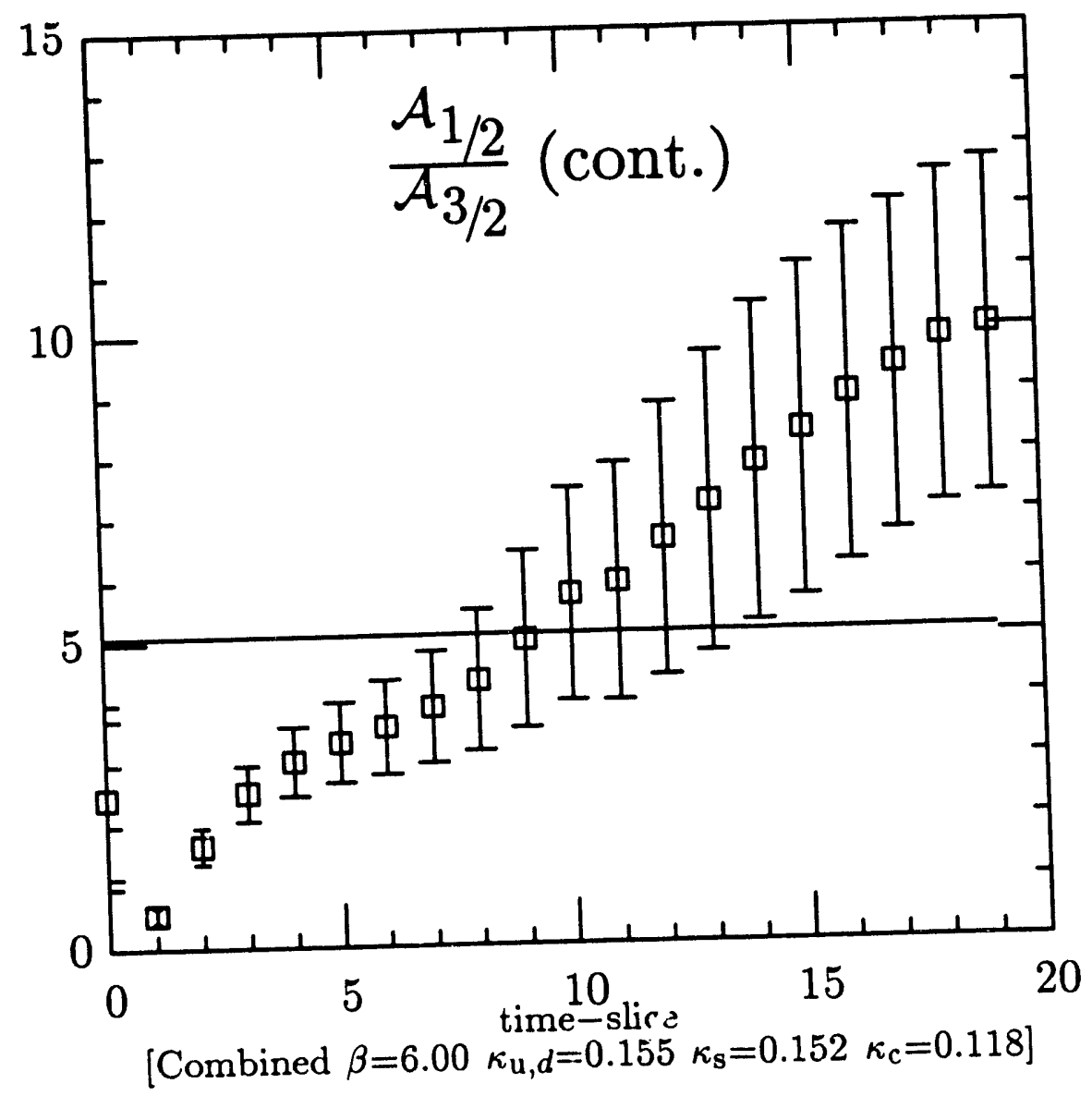

Figure I.19.1: Fit to $G_{1 / 2}^{(4)} / G_{3 / 2}^{(4)}$ versus $t_{K}$ for the combined $\beta=6.0$ lattices. 
I.20. $\mathcal{A}_{1 / 2}^{V S} / \mathcal{A}_{3 / 2}^{V S}$

\begin{tabular}{|c|c|c|c|c|}
\hline $\begin{array}{l}\text { Lattice } \\
\text { (configs) }\end{array}$ & $\begin{array}{l}\text { Model } \\
\text { t Range }\end{array}$ & $\begin{array}{l}a_{1} \\
a_{2} \\
a_{3} \\
\end{array}$ & Covar. Coeff. & $\frac{\chi^{2}}{n_{d}}$ \\
\hline \multicolumn{5}{|c|}{$\kappa_{u, d}=.155, \kappa_{s}=.152, \kappa_{c}=.118$} \\
\hline $\begin{array}{l}\text { Combined } \\
(27)\end{array}$ & $\begin{array}{c}I \\
{[12,16]}\end{array}$ & $\begin{array}{c}0.79(7) \\
0.0^{*} \\
0.0^{*}\end{array}$ & $\begin{array}{lll}1 & 0 & 0 \\
0 & 0 & 0 \\
0 & 0 & 0 \\
\end{array}$ & $\frac{7.9}{4}$ \\
\hline \multicolumn{5}{|c|}{$\kappa_{u, d}=.154, \kappa_{s}=.152, \kappa_{c}=.118$} \\
\hline $\begin{array}{l}\text { Combined } \\
(27)\end{array}$ & $\begin{array}{c}I \\
{[12,16]}\end{array}$ & $\begin{array}{c}0.93(4) \\
0.0^{*} \\
0.0^{*}\end{array}$ & $\begin{array}{lll}1 & 0 & 0 \\
0 & 0 & 0 \\
0 & 0 & 0 \\
\end{array}$ & $\frac{5.5}{4}$ \\
\hline \multicolumn{5}{|c|}{$\kappa_{u, d}=.152, \kappa_{s}=.152, \kappa_{c}=.118$} \\
\hline $\begin{array}{l}\text { Combined } \\
(27)\end{array}$ & $\begin{array}{c}I \\
{[12,16]}\end{array}$ & $\begin{array}{c}1.19(5) \\
0.0^{*} \\
0.0^{*}\end{array}$ & $\begin{array}{lll}1 & 0 & 0 \\
0 & 0 & 0 \\
0 & 0 & 0 \\
\end{array}$ & $\frac{2.1}{4}$ \\
\hline
\end{tabular}

Table 1.20.1: Fits to $G_{1 / 2}^{V S} / G_{3 / 2}^{V S}$ versus $t_{K}$ for the $\beta=6.0$ lattices. Model Function I is $f(t)=a_{1}+a_{2} t+a_{3} t^{2}$. Model Function II is $f(t)=a_{1}+a_{2} e^{a_{3} t}$. Parameters that are held fixed are followed by an asterisk. 


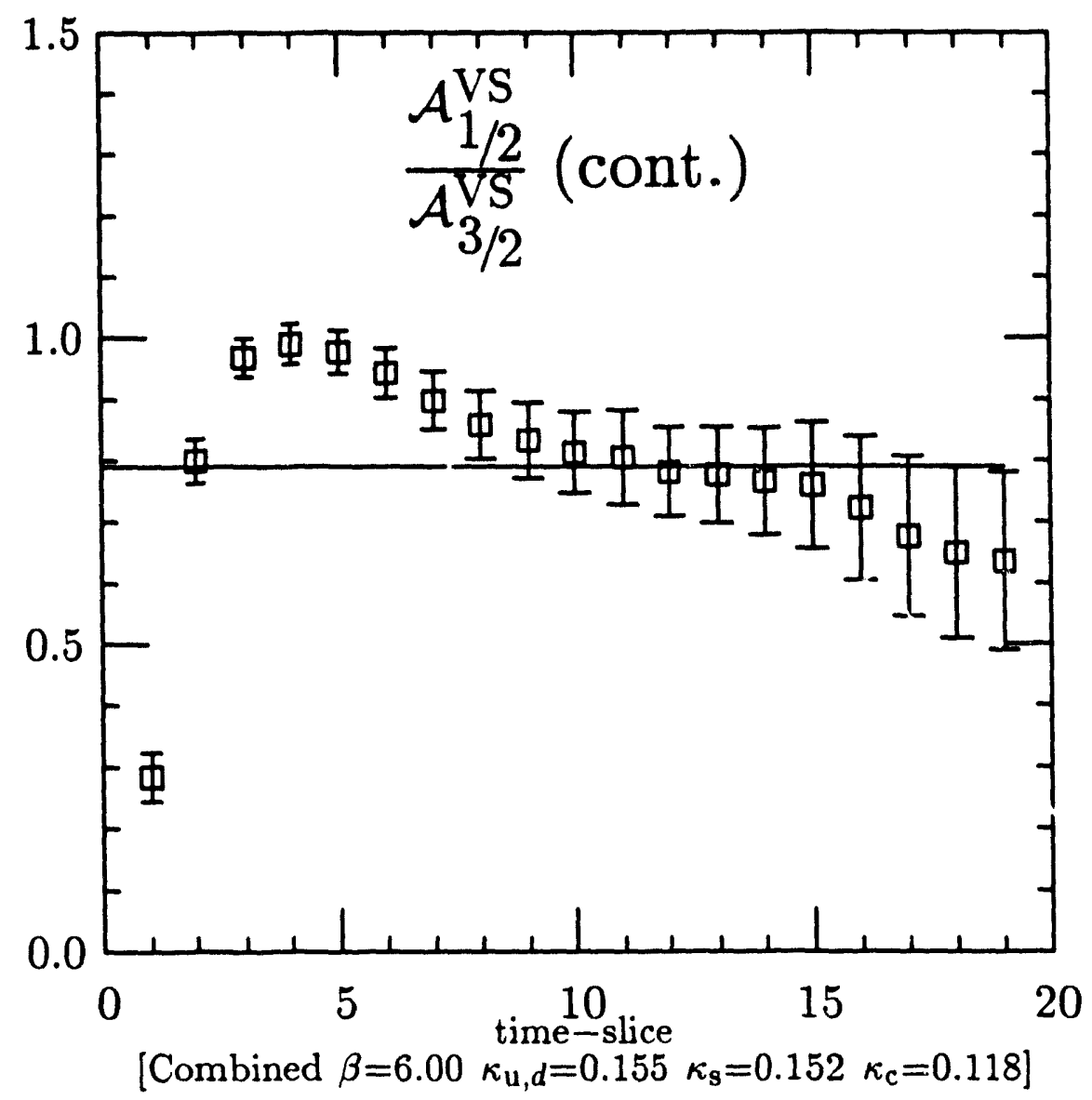

Figure 1.20.1: Fit to $G_{1 / 2}^{V S} / G_{3 / 2}^{V S}$ versus $t_{K}$ for the combined $\beta=6.0$ attices. 
1.21. $\mathcal{A}$ (fig8scalar $) / \mathcal{A}_{3 / 2}$

\begin{tabular}{|c|c|c|c|c|}
\hline $\begin{array}{l}\text { Lattice } \\
\text { (configs) }\end{array}$ & $\begin{array}{l}\text { Model } \\
\text { t Range }\end{array}$ & $\begin{array}{l}a_{1} \\
a_{2} \\
a_{3} \\
\end{array}$ & Covar. Coeff. & $\frac{\chi^{2}}{n_{d}}$ \\
\hline \multicolumn{5}{|c|}{$\kappa_{u, d}=.155, \kappa_{0}=.152, \kappa_{c}=.118$} \\
\hline $\begin{array}{l}\text { Combined } \\
\quad(27)\end{array}$ & $\begin{array}{c}I \\
{[9,14]}\end{array}$ & $\begin{array}{c}0.14(7) \\
0.0^{*} \\
0.0^{*} \\
\end{array}$ & $\begin{array}{lll}1 & 0 & 0 \\
0 & 0 & 0 \\
0 & 0 & 0 \\
\end{array}$ & $\frac{3.6}{5}$ \\
\hline \multicolumn{5}{|c|}{$\kappa_{u, d}=.154, \kappa_{s}=.152, \kappa_{c}=.118$} \\
\hline $\begin{array}{l}\text { Combined } \\
\quad(27)\end{array}$ & $\begin{array}{c}I \\
{[9,14]}\end{array}$ & $\begin{array}{c}0.10(4) \\
0.0^{*} \\
0.0^{*}\end{array}$ & $\begin{array}{lll}1 & 0 & 0 \\
0 & 0 & 0 \\
0 & 0 & 0 \\
\end{array}$ & $\frac{3.5}{5}$ \\
\hline \multicolumn{5}{|c|}{$\kappa_{u, d}=.152, \kappa_{s}=.152, \kappa_{c}=.118$} \\
\hline $\begin{array}{l}\text { Combined } \\
(27)\end{array}$ & $\begin{array}{c}I \\
{[9, .4]}\end{array}$ & $\begin{array}{c}0.058(21) \\
0.0^{*} \\
0.0^{*}\end{array}$ & $\begin{array}{lll}1 & 0 & 0 \\
0 & 0 & 0 \\
0 & 0 & 0\end{array}$ & $\frac{4.1}{5}$ \\
\hline
\end{tabular}

Table 1.21.1: Fits to TI ratio for fig8scalar $/ G_{3 / 2}^{(4)}$ versus $t_{K}$ for the $\beta=6.0$ lattices. Model Function I is $f(t)=a_{1}+a_{2} t+a_{3} t^{2}$. Model Function II is $f(t)=a_{1}+a_{2} e^{a_{3} t}$. Parameters that are held fixed are followed by an asterisk. 


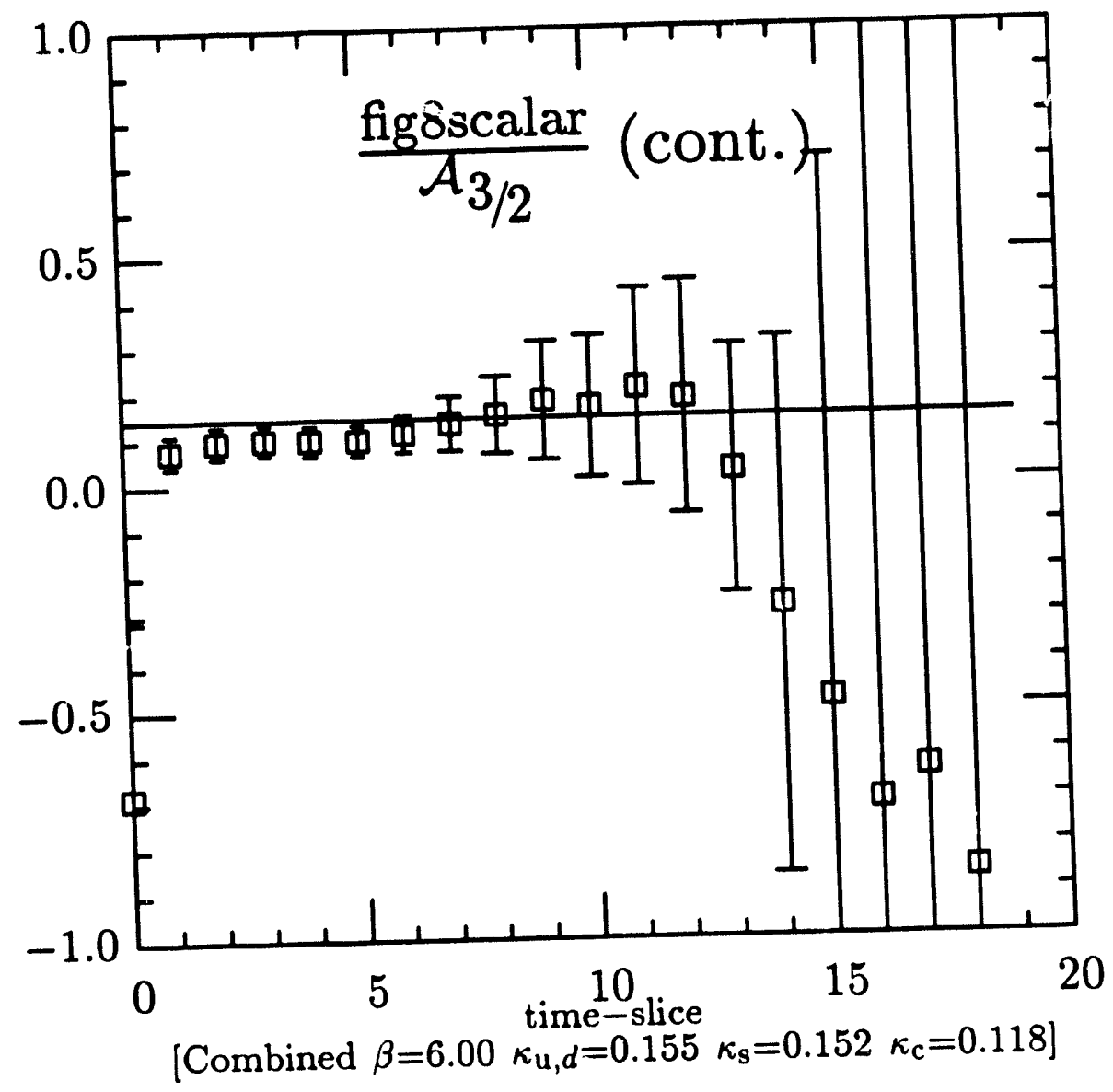

Figure I.21.1: Fit to TI ratio for fig8scalar $/ G_{3 / 2}^{(4)}$ versus $t_{K}$ for the combined $\beta=6.0$ lattices. 


\subsection{Effective $1 / \mathrm{N}$}

\begin{tabular}{|c|c|c|c|c|}
\hline $\begin{array}{l}\text { Lattice } \\
\text { (configs) }\end{array}$ & $\begin{array}{l}\text { Model } \\
\text { t Range }\end{array}$ & $\begin{array}{l}a_{1} \\
a_{2} \\
a_{3} \\
\end{array}$ & Covar. Coeff. & $\frac{\chi^{2}}{n_{d}}$ \\
\hline \multicolumn{5}{|c|}{$\kappa_{u, d}=.155, \kappa_{,}=.152, \kappa_{c}=.118$} \\
\hline $\begin{array}{l}\text { Combined } \\
(27)\end{array}$ & $\begin{array}{c}I \\
{[12,16]}\end{array}$ & $\begin{array}{c}-0.24(7) \\
0.0^{*} \\
0.0^{*} \\
\end{array}$ & $\begin{array}{lll}1 & 0 & 0 \\
0 & 0 & 0 \\
0 & 0 & 0 \\
\end{array}$ & $\frac{0.95}{4}$ \\
\hline \multicolumn{5}{|c|}{$\kappa_{u, d}=.154, \kappa_{s}=.152, \kappa_{c}=.118$} \\
\hline $\begin{array}{l}\text { Combined } \\
(27)\end{array}$ & $\begin{array}{c}I \\
{[12,16]}\end{array}$ & $\begin{array}{c}-0.32(9) \\
0.0^{*} \\
0.0^{*}\end{array}$ & $\begin{array}{lll}1 & 0 & 0 \\
0 & 0 & 0 \\
0 & 0 & 0 \\
\end{array}$ & $\frac{0.48}{4}$ \\
\hline \multicolumn{5}{|c|}{$\kappa_{u, d}=.152, \kappa_{s}=.152, \kappa_{c}=.118$} \\
\hline $\begin{array}{c}\text { Combined } \\
\quad(27)\end{array}$ & $\begin{array}{c}I \\
{[12,16]}\end{array}$ & $\begin{array}{c}3.4(87) \\
0.0^{*} \\
0.0^{*}\end{array}$ & $\begin{array}{lll}1 & 0 & 0 \\
0 & 0 & 0 \\
0 & 0 & u\end{array}$ & $\frac{0.10}{4}$ \\
\hline
\end{tabular}

Table 1.22.1: Fits to effective $1 / \mathrm{N}$ versus $t_{K}$ for the $\beta=6.0$ lattices. Model Function I is $f(t)=a_{1}+a_{2} t+a_{3} t^{2}$. Model Function II is $f(t)=a_{1}+a_{2} e^{a_{3} t}$. Parameters that are held fixed are followed by an asterisk. 


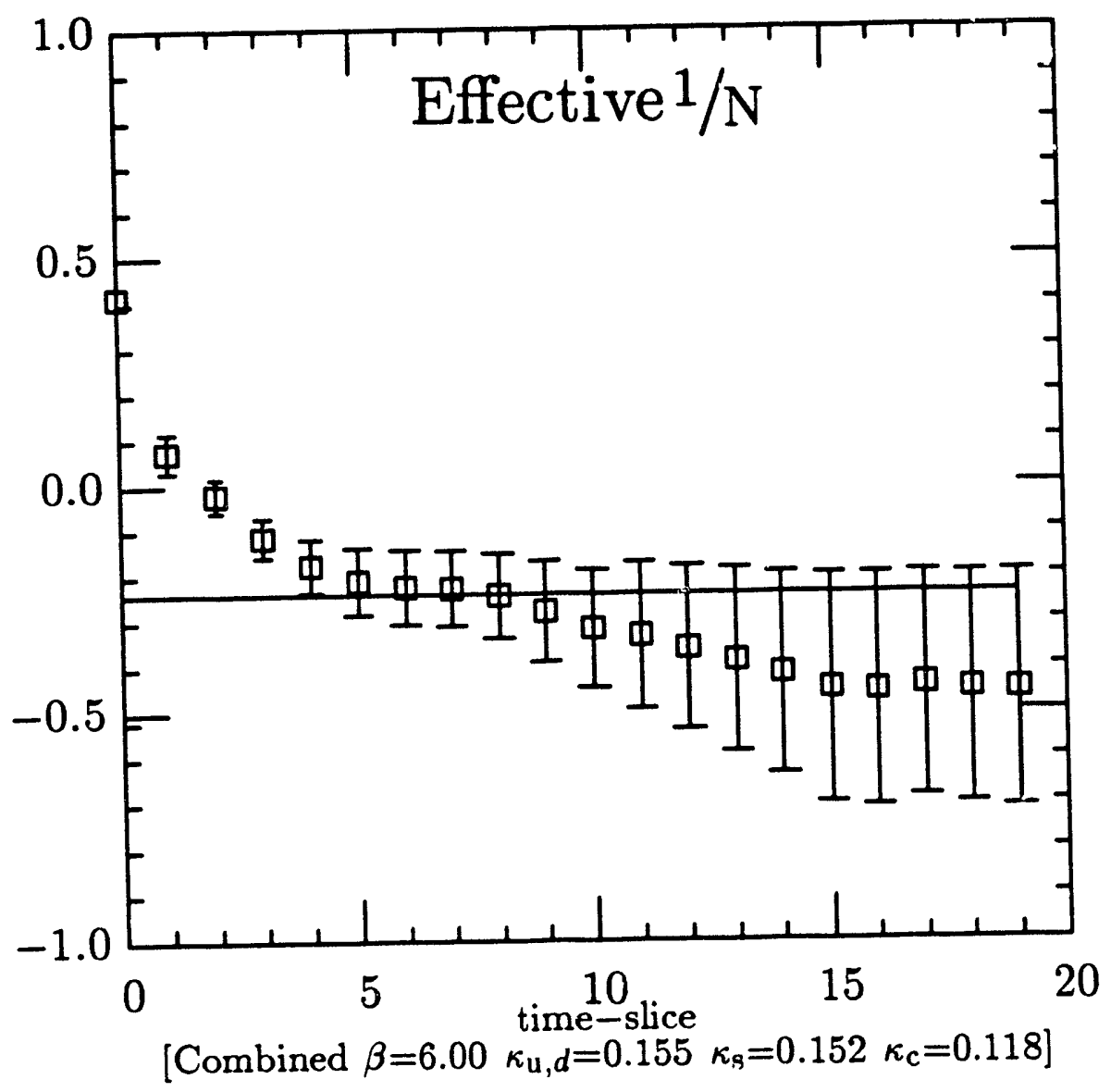

Figure 1.22.1: Fits to effective $1 / \mathrm{N}$ versus $t_{K}$ for the combined $\beta=6.0$ lattices. 


\section{Appendix II. \\ Numerical Infrastructure}

\section{II.1. Data Ordering}

Quantities of interest in lattice gauge theory are typically complex valued functions of space-time indices $x, y, z$, and $t$ along with various "internal" degrees of freedom such as spin, color and direction. The results of Monte Carlo simulations are numerical approximations to these functions computed and stored as complex floating-point data type arrays indexed by the discrete set of space-time points and the values of any internal degrees of freedom.

These multidimensional arrays must then be mapped into the linear address space of a computer. All multidimensional data arrays are stored in FORTRAN ordering. A reference to an array $q$ with two internal degrees of freedom $s$ and $c$ is denoted in FORTRAN as $\mathrm{q}(\mathrm{s}, \mathrm{c}, \mathrm{x}, \mathrm{y}, \mathrm{z}, \mathrm{t})$. As one moves through the linear address space mapping of this array, it is $\mathrm{s}$ the left-most index that changes fastest while the right-most index $\mathrm{c}$ that changes slowest.

There are two orderings used for data arrays. Consider some quantity $v$ with internal degrees of freedom $i_{1}, \ldots, i_{n}$ and that it depends on the space-time point. Assume that the range of coordinates for space-time lattice points is $0 \leq x, y, z<$ $n_{s}-1$ and $0 \leq t<n_{t}-1$ so that the lattice is of size $n_{s}^{3} \times n_{t}$.

The first possible ordering is time-slice ordering in which the array is written in FORTRAN notation as $v\left(i_{1}, \ldots, i_{n}, x, y, z, t\right)$. The time index being the slowest changing index means that it is convenient to process the data piecewise by taking a "slice" through it with all data values in a slice having a fixed time value. 
The second ordering that is used is hyperplane ordering. Each space-time point $(x, y, z, t)$ is assigned to a hyperplane $p$ by the rule $p=x \oplus y \oplus z \oplus t$ where the operation $\oplus$ is modular addition with $0 \leq p<n_{s}$ and all quantities will have periodic boundary conditions in the spatial directions. There are then $n_{s}$ hyperplanes each hyperplane containing $n_{s}^{2} \times n_{t}$ space-time points.

In FORTRAN style array notation the hyperplane ordered quantity $\mathrm{v}$ is written $v\left(i_{1}, \ldots, i_{n}, y, z, t, p\right)$. The hyperplane index now being the last index, and thus the one that changes slowest, makes it convenient to manipulate slices of data that all belong to the same hyperplane. Placing the data in hyperplane order has the desirable feature all the nearest neighbors to a given point on hyperplane $p$ are located on either the preceding hyperplane $p^{\prime}=p \ominus 1$ or succeeding hyperplane $p^{\prime}=$ $p \oplus 1$. Where $\oplus$ and $\ominus$ are modular addition and subtraction on the range $\left[0, n_{s}-1\right]$ For points on the time boundaries $t=0$ and $t=n_{t}-1$, the nearest neighbor condition may be modified depending on whether periodic or perfectly absorbing boundary conditions are desired. The boundary conditions are summarized for FORTRAN style arrays in the table:

\begin{tabular}{|c|c|c|}
\hline direction & \multicolumn{2}{|c|}{ nearest neighbor to $v\left(i_{1}, \ldots, i_{n}, y, z, t, p\right)$} \\
\hline$\mu$ & $x+\hat{\mu}$ & $x-\hat{\mu}$ \\
\hline 1 & $\mathrm{v}\left(\mathrm{i}_{1}, \ldots, \mathrm{i}_{\mathrm{n}}, \mathrm{y}, \mathrm{z}, \mathrm{t}, \mathrm{p} \oplus 1\right)$ & $\mathrm{v}\left(\mathrm{i}_{1}, \ldots, \mathrm{i}_{\mathrm{n}}, \mathrm{y}, \mathrm{z}, \mathrm{t}, \mathrm{p} \ominus 1\right)$ \\
\hline 2 & $\mathrm{v}\left(\mathrm{i}_{1}, \ldots, \mathrm{i}_{\mathrm{n}}, \mathrm{y} \oplus 1, \mathrm{z}, \mathrm{t}, \mathrm{p} \oplus 1\right)$ & $\mathrm{v}\left(\mathrm{i}_{1}, \ldots, \mathrm{i}_{\mathrm{n}}, \mathrm{y} \ominus 1, \mathrm{z}, \mathrm{t}, \mathrm{p} \ominus 1\right)$ \\
\hline 3 & $\mathrm{v}\left(\mathrm{i}_{1}, \ldots, \mathrm{i}_{\mathrm{n}}, \mathrm{y}, \mathrm{z} \oplus 1, \mathrm{t}, \mathrm{p} \oplus 1\right)$ & $\mathrm{v}\left(\mathrm{i}_{1}, \ldots, \mathrm{i}_{\mathrm{n}}, \mathrm{y}, \mathrm{z} \Theta 1, \mathrm{t}, \mathrm{p} \ominus 1\right)$ \\
\hline 4 & $\mathrm{v}\left(\mathrm{i}_{1}, \ldots, \mathrm{i}_{\mathrm{n}}, \mathrm{y}, \mathrm{z}, \mathrm{t} \oplus 1, \mathrm{p} \oplus 1\right)$ & $\mathrm{v}\left(\mathrm{i}_{1}, \ldots, \mathrm{i}_{\mathrm{n}}, \mathrm{y}, \mathrm{z}, \mathrm{t} \ominus 1, \mathrm{p} \ominus 1\right)$ \\
\hline
\end{tabular}

Having the nearest neighbors to a point on hyperplanes different than the hyperplane of the point has numerical advantages. Programs must be written to use vectorization to take full advantage of the number-crunching ability of supercomputers. When computations are vectorized pipelining no longer guarantees the updating of a memory location with a new result will occur before that datum is 
needed for a future operation. Compilers are usually good at detecting such data dependencies and will refuse to vectorize program segments when such dependencies exist. The algorithms used in this lattice gauge theory work to compute some quantity at a given point only depend on quantities at that point and quantities at the nearest-neighbor points. Mathematical operations performed on a hyperplane at a time should then vectorize.

\section{II.2. Gauge Conflgurations}

A typical gauge link variable $U_{\mu}(x)$ is a complex $3 \times 3$ matrix that depends on link direction $\mu$ and space-time coordinates $x, y, z, t$. The configuration of these gauge fields can be stored in several formats:

\begin{tabular}{|c|c|}
\hline $\begin{array}{l}\text { data format } \\
\text { data file name }\end{array}$ & description \\
\hline $\begin{array}{l}\mathrm{u}(\text { row }, \text { col }, \mu, \mathrm{x}, \mathrm{y}, \mathrm{z}, \mathrm{t}) \\
\mathrm{u}<\text { lat }>\mathrm{n}<\text { config }>\end{array}$ & $\begin{array}{l}\text { Time-slice ordered. The full complex } 3 \times 3 \text { matrices } \\
\text { stored row } \times \text { col. }\end{array}$ \\
\hline $\begin{array}{r}\mathrm{u}(2, \text { row }, \text { col }, \mathrm{x}, \mathrm{y}, \mathrm{z}, \mathrm{t}) \\
\mathrm{ub}<\mathrm{lat}>\mathrm{n}<\text { config }>\end{array}$ & $\begin{array}{l}\text { Time-slice ordered. Packed } 2 \times 3 \text { matrices stored } \\
\text { row } \times \text { col. The third column is dropped. Real and } \\
\text { imaginary parts for all four directions packed as scaled } \\
\text { integers in two Cray words. }\end{array}$ \\
\hline $\begin{array}{l}\mathrm{u}(2, \text { row }, \text { col, }, \bar{x}, \bar{y}, \bar{z}, t) \\
\text { ua }<\text { lat }>\text { n }<\text { config }>\end{array}$ & $\begin{array}{l}\text { Time-slice ordered. Packed } 2 \times 3 \text { satrices like "ub" } \\
\text { format except spatial indices have been shifted: } U \\
\text { matrix at space-time coordinate } 2 \text { in direction } \mu \neq 4 \\
\text { is located at } \bar{x}=x+\hat{\mu} \text {. }\end{array}$ \\
\hline $\begin{array}{c}\mathrm{u}(2, \text { row }, \text { col }, \mathrm{y}, \mathrm{z}, \mathrm{t}, \mathrm{p}) \\
\mathrm{uhy}<\text { lat }>\text { n }<\text { config }>\end{array}$ & $\begin{array}{l}\text { Hyperplane ordered. Packed } 2 \times 3 \text { matrices where the } \\
\text { third row is dropped. Real and imaginary parts for all } \\
\text { four directions packed as scaled integers in two Cray } \\
\text { words. }\end{array}$ \\
\hline
\end{tabular}

As part of the packing of the $U$ matrices the last row of each matrix is dropped. The whole $U$ matrix can be reconstructed by using the property that for $S U(3)$ matrices

$$
U_{3 i}=\epsilon_{i j k} U_{1 j}^{*} U_{2 k}^{*}
$$


which is in the form of a vector cross product when the rows of the matrix $U$ are considered vectors. Optimally, the $U$ matrices can be parameterized in terms of just nine real parameters. Instead of twelve parameters of the method above. The method above while not optimal is easy to implement.

The real and imaginary elements of the $U$ matrices for all four link directions $\mu$ are separately packed into two 64-bit Cray words:

\begin{tabular}{|c|c|c|c|}
\hline $\mathbf{R e}_{1}$ & $\operatorname{Re}_{2}$ & $\mathrm{Re}_{3}$ & $\mathrm{Re}_{4}$ \\
\hline addr+1: & & & \\
\hline $\operatorname{Im}_{1}$ & $\operatorname{Im}_{2}$ & $\mathrm{Im}_{3}$ & $\operatorname{Im}_{4}$ \\
\hline
\end{tabular}

where $\operatorname{Re}_{\mu}$ and $\operatorname{Im}_{\mu}$ represent the real and imaginary parts of the the $U$ matrix element in the $\mu$ direction.

Since each packed number $\eta$ is the real or imaginary part of an $S U(3)$ matrix it is bounded by the range $-1 \leq \eta \leq 1$. Since the numbers have limited range storing them in a general floating-point format may be wasteful if the floatingpoint format is capable of representing a much wider dynamic range than $[-1,1]$. Instead, the numbers are stored as scaled integer values. Notice that $0 \leq \eta+1 \leq 2$ so only values in this range need be represented. The floating-point values $\eta$ can then be represented by 16 -bit integers by the mapping $[-1,1] \mapsto\left[0,2^{15}\right]$. The mapping is realized by converting $\eta \times 2^{14}$ to the nearest integer and then adding $2^{14}$.

The precision of scaled integer packing with the last row dropped has been checked by comparing element-by-element a packed and then subsequently unpacked configuration of a $24^{4}$ lattice at $\beta=6.3$ against the original full precision configuration. The magnitude of the elements peaks sharply near one as is expected for weak coupling. The absolute error in all numbers was less than $10^{-3}$ 
with an rms value of $1.8 \times 10^{-5}$. The most probable relative error is about $5 \times 10^{-4}$ with an rms value of .19 . The relative error distribution is skewed by a relatively few but large relative errors that occur for very small floating-point values that happen to be in the third (dropped) row.

The parameter <lat $>$ in the file name structures tabulated above is assigned to each set of gauge configurations for a given lattice size and $\beta$. The value of this parameter for some of the more recent lattices are:

\begin{tabular}{|c|c|c|c|l|}
\hline$\beta$ & $n_{,}^{3} \times n_{t}$ & configs & <lat $>$ & \multicolumn{1}{|c|}{ description } \\
\hline 6.4 & $32^{3} \times 48$ & $>8$ & $\mathrm{p} 64, \mathrm{c} 64$ & $\begin{array}{l}\text { underway now. 'c' set are fixed in } \\
\text { coulomb gauge. }\end{array}$ \\
\hline 6.0 & $24^{3} \times 40$ & 8 & 160 & fixed to landau gauge \\
\hline 6.0 & $16^{3} \times 40$ & 19 & 160 & fixed to landau gauge \\
\hline 5.7 & $16^{3} \times 32$ & 11 & $\mathrm{z} 57$ & not gauge fixed \\
\hline 5.7 & $16^{3} \times 21$ & 5 & $\mathrm{v} 57$ & combined with above to give 16 configs \\
\hline 5.7 & $16^{3} \times 24$ & 16 & $\mathrm{y} 57$ & not gauge fixed \\
\hline
\end{tabular}

Each configuration is stored in a separate file with the parameter <config $>$ identifying the configuration. In most cases this parameter is the cumulative number of Monte Carlo sweeps through the lattice for the sampled configuration divided by one thousand.

As an example of these naming conventions, the file containing the configuration in landau gauge generated after 8,000 Monte Carlo sweeps on the $24^{3} \times 40$ lattice at $\beta=6.0$ and stored in "ua" format would be named ual60n08. 


\section{II.3. Conflguration Generation}

The number of gauge degrees of freedom on even a lattice of modest size is extremely large. For example, on a $10^{4}$ lattice there are approximately $3.6 \times 10^{5}$ degrees of freedom. To evaluate an expectation value of some physical quantity one must integrate over these degrees of freedom. It is obviously impractical for a computer to all these integrals by direct methods. Fortunately, this is not necessary. The needed integrals can be approximated to a given precision by Monte Carlo methods.

In the quenched approximation the expectation value of some physical quantity $\theta$ is given by

$$
<\theta>=\frac{1}{Z} \int \mathcal{D} \mathcal{U} \theta(\mathcal{U}) e^{-\beta S_{g}(u)}
$$

with

$$
Z=\int \mathcal{D U} e^{-\beta S_{g}(\mathcal{U})}
$$

These multiple integrals can be approximated by a sum over configurations

$$
<\theta>=\frac{1}{N} \sum_{\substack{i=1 \\ u_{i} \in\{U\}}}^{N} \theta\left(\mathcal{U}_{i}\right)
$$

where a configuration is defined as a collection of values picked for all of the gauge degrees of freedom. The probability of a given configuration $\mathcal{U}$ appearing in the set of configurations $\{\mathcal{U}\}$ that is averaged over is now proportional to

$$
P(\mathcal{U}) \mathcal{D U}=\frac{1}{Z} e^{-\beta S_{g}(\mathcal{U})} \mathcal{D U}
$$

which provides the appropriate weighting factor in the sum that corresponds to the Boltzmann weighting factor in the original integral. In the process of importance 
sampling one attempts to pick a set of sictistically independent configurations with the appropriate weight from the universe of all possible configurations.

The method used to generate these configurations is the Kennedy Pendleton algorithm $\mathrm{m}^{50}$ a computationally more efficient version of the Creutz Heat Bath method $^{51}$. In these methods $S U(2)$ matrices $V$ are generated with probability proportional to

$$
P(V) d V=\frac{1}{Z^{\prime}} e^{-\beta S_{S U(2)}(V)}
$$

Following Cabbibo and Marinari ${ }^{52}$, these pseudorandomly generated $S U(2)$ matrices are used to update a $S U(3)$ gauge matrix $U$ on some link. Three such $2 \times 2 S U(2)$ matrices are generated for a link and are then promoted to $3 \times 3$ matrices in a way that each will thermalize one of three $S U(2)$ subgroups of the $S U(3)$ link matrix when multiplied into the link matrix. If $V^{1}, V^{2}$, and $V^{3}$ represent the three $S U(2)$ matrices then the new link matrix is given by:

$$
U_{\text {new }}=\left(\begin{array}{ccc}
V_{11}^{3} & V_{12}^{3} & 0 \\
V_{21}^{3} & V_{22}^{3} & 0 \\
0 & 0 & 1
\end{array}\right)\left(\begin{array}{ccc}
1 & 0 & 0 \\
0 & V_{11}^{2} & V_{12}^{2} \\
0 & V_{21}^{2} & V_{22}^{2}
\end{array}\right)\left(\begin{array}{ccc}
V_{11}^{1} & 0 & V_{12}^{1} \\
0 & 1 & 0 \\
V_{21}^{1} & 0 & V_{22}^{1}
\end{array}\right) U_{\text {old }}
$$

In one Monte Carlo "sweep" of the lattice all link variables are updated by this scheme.

Configuration generation is started with all link matrices set equal to the identity. The lattice must then be allowed to reach thermal equilibrium by doing several thousand sweeps. After attaining thermal equilibrium, representative configurations are selected for the set of configurations to averaged over by periodically saving the configuration generated after some large number of sweeps. The number of sweeps between samples must be large enough to reduce statistical correlations among sampled configurations. 


\section{II.4. Quark Propagators}

Quark propagator files can also be either time-slice or hyperplane ordered. Time-slice ordering is preferred for long term storage since most physics analysis is done with the time coordinates as the variables while propagators may be in hyperplane order while being generated.

The FORTRAN style data array ordering for data such as quark propagators $Q_{\mu \nu}^{a b}(x)$ that carries the four indices $\mu, a$ the destination spin and color and $\nu, b$ the source spin and color indices and which depends on space-time point $x$ is:

\begin{tabular}{|l|l|}
\hline $\begin{array}{l}\text { data format } \\
\text { data file name }\end{array}$ & \multicolumn{1}{|c|}{ description } \\
\hline $\begin{array}{l}\mathrm{Q}(3, \mathrm{a}, \mathrm{x}, \mathrm{y}, \mathrm{z}, \mathrm{t}, \mathrm{b}, \nu) \\
<\text { type }><\text { config }><\kappa>\end{array}$ & $\begin{array}{l}\text { Time-slice ordered. Eight into three packed floating- } \\
\text { point format. Data values for the destination spin } \mu \\
\text { are packed into three Cray words. }\end{array}$ \\
\hline $\begin{array}{l}\mathrm{Q}(3, \mathrm{a}, \mathrm{y}, \mathrm{z}, \mathrm{t}, \mathrm{p}, \mathrm{b}, \nu) \\
\text { none standard }\end{array}$ & Hyperplane ordered. Packing same as above. \\
\hline
\end{tabular}

The storage method used called eight into three packing takes eight 64-bit floating-point data values (or four complex values) and stores them in a 24-bit floating-point representation:

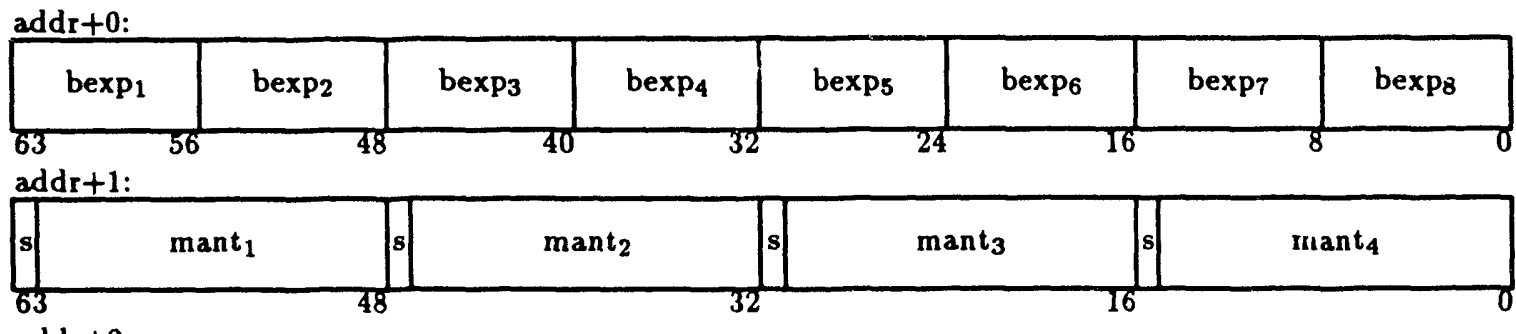

addr+2:

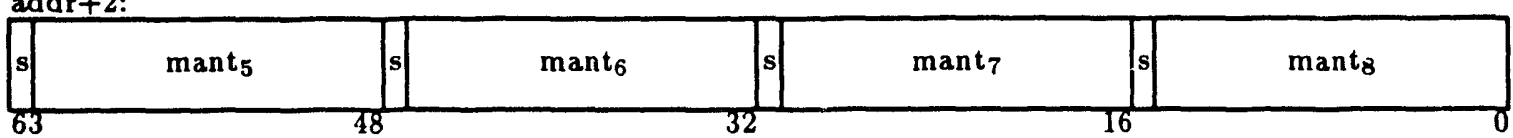

Where the packed numbers 1 and 2 are the real and imaginary values for spin component $\mu=1$ and so on up to pac' ed numbers 7 and 8 which together represent the complex value at $\mu=4$. 
A packed floating-point number consist of an eight-bit biased exponent stored at relative word address 0 and the mantissa sign bit followed by the 15-bit mantissa stored in one of the next two words. The $\mathrm{n}^{\text {th }}$ floating-point value is given by $x_{n}=(-1)^{s_{n}} \times$ mant $_{n} \times 2^{\text {bexpn-bias }}$. The exponent bias is 128 (decimal). The mantissa has the form $.1 b b b b b b b b b b b b b b$ (binary) where each $b$ represents one binary digit. The radix point precedes the most significant bit which is always 1 for non-zero numbers. The Cray floating-point mantissa is rounded to the nearest 15-bit value before being truncated. Floating-point zero is represented by all 24-bits zero. The representable magnitudes are approximately within the range $2.9 \times 10^{-39} \lesssim x_{n} \lesssim 1.7 \times 10^{38}$. Overflowing magnitudes are truncated to the largest representable magnitude while underflowing magnitudes become zero. Packed values retain about 4.5 significant digits of precision.

The format for quark propagator file names is $\langle$ type $\rangle\langle$ config $\rangle\langle\kappa\rangle$ where the parameter <config $>$ is the two character configuration name that matches the $<$ config $>$ parameter in the file name of the corresponding background gauge configuration used in computing the propagator. The parameter $\langle\kappa\rangle$ is a character string representing the hopping constant $\kappa$ of the quark. The leading decimal point is dropped.

The parameter <type> indicates the type of propagator. This parameter indicates whether the quark propagator is an ordinary propagator with hopping constant $\kappa$ from some source at the origin to every other space-time point $x$ :

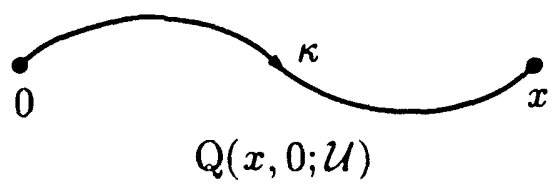


or if it is an "exponentiated" propagator meaning that a quark propagator with hopping constant $\kappa^{\prime}$ that starts from the origin and goes to some point on timeslice $t^{\prime}$ where there is an operator insertion $\Gamma$ it then continues on as a propagator with hopping constant $\kappa$ from $t^{\prime}$ to space-time point $x$ :

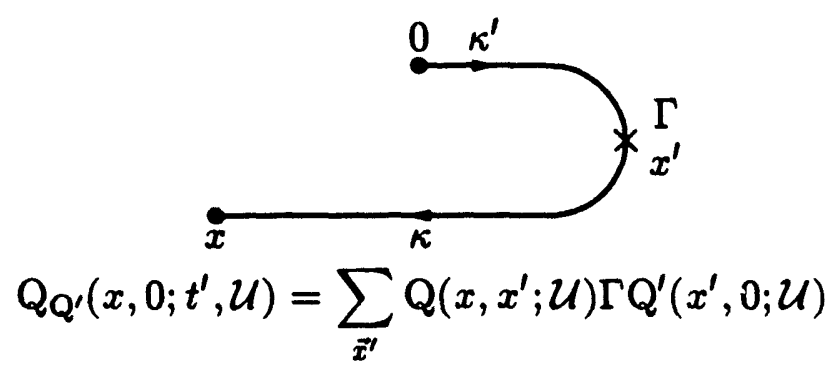

For exponentiated propagators the parameter <type $>$ indicates the hopping constant $\kappa^{\prime}$ of the quark propagator from the origin and the time-slice $t^{\prime}$ of the operator insertion $\Gamma$. All exponentiated propagators generated to the present time have been with $\Gamma=\gamma^{5}$.

For $\beta=6.0$ lattices the exponentiations have been carried out at $t^{\prime}= \pm 11$ with a "strange" quark of $\kappa=.152$ and "charm" as $\kappa=.118$ or .135 . No exponentiation means a standard propagator and degenerate exponentiation is with $\kappa^{\prime}=\kappa$. The table summarizes:

\begin{tabular}{|c|c|c|l|}
\hline <type & $\kappa^{\prime}$ & $t^{\prime}$ & exponentiation \\
\hline ga & - & - & none \\
\hline ha & $.152 ?$ & -11 & strange \\
\hline ja & $.152 ?$ & +11 & strange \\
\hline ma & .118 & -11 & charm \\
\hline na & .118 & +11 & charm \\
\hline oa & .135 & -11 & charm \\
\hline pa & .135 & +11 & charm \\
\hline ra & $\kappa$ & +11 & degenerate \\
\hline va & $\kappa$ & -11 & degenerate \\
\hline
\end{tabular}


For $\beta=5.7$ lattices exponentiation has been carried out at $t^{\prime}= \pm 7$ with $\kappa=.160$ as "strange" and $\kappa=.094$ as "charm":

\begin{tabular}{|c|c|c|l|}
\hline <type $>$ & $\kappa^{\prime}$ & $t^{\prime}$ & exponentiation \\
\hline ga & - & - & none \\
\hline ha & .160 & -7 & strange \\
\hline ja & .160 & +7 & strange \\
\hline ma & .094 & -7 & charm \\
\hline na & .094 & +7 & charm \\
\hline ra & $\kappa$ & +7 & degenerate \\
\hline va & $\kappa$ & -7 & degenerate \\
\hline
\end{tabular}

\section{II.5. Propagator Extrapolation}

Quark propagator calculations typically consume approximately $80 \%$ of the computer resources devoted to this project. This being the case any speedup that can be achieved can have an enormous impact.

Propagaiors are iteratively calculated by sparse matrix inversion techniques. The matrix to be inverted $\mathrm{Q}^{-1}$ can be written in the form $\mathrm{Q}^{-1}=I-\kappa D$ where $I$ is the identity matrix $\kappa$ is the hopping constant and $D$ is a matrix with off-diagonal elements. As $\kappa$ becomes smaller (heavier quarks) the off-diagonal elements become smaller and the elements near the diagonal become more important. The matrix inversion algorithms then typically converge to a solution in a fewer number of iterations. Conversely, as the hopping constant increases towards the critical value of $\kappa$ at which the pseudoscalar quark bound state becomes massless, the number of iterations required for convergence rapidly increases.

Since the propagators for the heavier quarks are in relative terms easier to calculate than propagators for lighter quarks, it would be advantageous to calculate the heavier quark propagators first if one could make use of the propagators for 
heavier quarks to decrease the effort involved in calculating propagators for the lighter quarks. The simplest way to do this is for a given gauge configuration to use the previously generated quark propagator that is closest in $\kappa$ to the quark propagator to be generated as the starting guess input to the matrix inverter.

It is possible to even do betier than this by taking as the initial guess for the propagator an extrapolation based on several previously computed propagators with different $\kappa$ values.

the extrapolation is done using a polynomial interpolating function. Consider the propagator $\mathrm{Q}$ to be a function of $\kappa$. Suppose propagators have been calculated at $n$ different values of the hopping constant. The guess for the new propagator is taken to be

$$
\mathrm{Q}(\kappa)_{g u e s s}=\sum_{i=1}^{n} \mathrm{Q}\left(\kappa_{i}\right) \prod_{\substack{j=1 \\ j \neq i}}^{n} \frac{\left(\kappa-\kappa_{j}\right)}{\left(\kappa_{i}-\kappa_{j}\right)}
$$

which is the Lagrange interpolating function. It is the unique $(n-1)^{\text {th }}$ order polynomial that takes as its value $\mathrm{Q}\left(\kappa_{i}\right)$ at $\kappa=\kappa_{i}$.

Such a polynomial is not normally a good estimate of the behavior of the function much outside the range of interpolation. In many instances it may be better to choose a method such as a spline fit which emphasizes smoothness of the resulting polynomial approximation by using polynomials of a lower degree. Lagrange interpolation, when compared to the previous method of using the propagator closest in $\kappa$ as the starting guess is found to significantly reduce the number of matrix inverter iterations to achieve a given convergence criterion even though it may not be the extrapolation method of choice. 


\section{II.6. Matrix Inversion Algorithms}

Quark propagators are iteratively calculated using matrix inversion techniques for sparse matrices. The matrix to be inverted is

$$
\mathrm{Q}^{-1}\left(x_{1}, x_{2} ; \mathcal{U}\right)=I\left(x_{1}, x_{2}\right)-\kappa D\left(x_{1}, x_{2} ; \mathcal{U}\right)
$$

where $I$ is the unit matrix and

$$
\begin{aligned}
& D\left(x_{1}, x_{2} ; \mathcal{U}\right)= \\
& \sum_{\mu=1}^{4}\left\{\left(1-\gamma^{\mu}\right) U_{\mu}\left(x_{1}\right) \delta_{x_{1}+\hat{\mu}, x_{2}}+\left(1+\gamma^{\mu}\right) U_{\mu}^{\dagger}\left(x_{2}\right) \delta_{x_{1}-\hat{\mu}, x_{2}}\right\}
\end{aligned}
$$

is the matrix of the off-diagonal elements. The spin and color indices are suppressed in the expressions above. Taking these degrees of freedom into account the above matrix is of dimensions $4 \cdot 3 \cdot n_{s}^{3} \cdot n_{t} \times 4 \cdot 3 \cdot n_{s}^{3} \cdot n_{t}$. Fortunately most of the elements are zero!

The general problem to be solved is find $\phi\left(x_{2}\right)$ given some source function $\omega\left(x_{1}\right)$ where

$$
\mathrm{Q}^{-1}\left(x_{1}, x_{2} ; \mathcal{U}\right) \phi\left(x_{2}\right)=\omega\left(x_{1}\right)
$$

once again all explicit spin and color indices are suppressed and there is an implicit sum over index $x_{2}$.

To find the propagator from the origin to every point on the lattice take $\omega\left(x_{1}\right)=$ $\delta\left(x_{1}, 0\right)$. A wall source at time-slice $t^{\prime}$ is implemented by taking $\omega\left(x_{1}\right)=\delta\left(t_{1}, t^{\prime}\right)$. For a propagator exponentiated at time-slice $t^{\prime}$ with operator insertion $\Gamma$ take the source function to be $\omega\left(x_{1}\right)=\Gamma Q^{\prime}\left(x_{1}, 0 ; \mathcal{U}\right) \delta\left(t_{1}, t^{\prime}\right)$.

There are several matrix inversion algorithms commonly used to solve these equations. The simplest but far from optimum method is simple Jacobi iteration: 
1. Construct an initial guess for the solution: $\phi_{0}\left(x_{2}\right)$.

2. Compute the residue vector:

$$
r_{n}\left(x_{1}\right)=\mathrm{Q}^{-1}\left(x_{1}, x_{2} ; \mathcal{U}\right) \phi_{n}\left(x_{2}\right)-\omega\left(x_{1}\right)
$$

3. Compute a new guess for the solution:

$$
\phi_{n+1}\left(x_{1}\right)=\phi_{n}\left(x_{1}\right)-\alpha r_{n}\left(x_{1}\right)
$$

where $\alpha=1$.

4. Repeat steps two and three until $\left|r_{n}\left(x_{1}\right)\right|<\epsilon$ where $\epsilon$ is some convergence criterion.

Where the parameter $\alpha$ can be adjusted to a value that speeds up convergence.

The Gauss-Seidel method is a variation upon the method above. In this method steps 2 and 3 are carried out together. The elements of $\phi_{n}$ are updated to their new values $\phi_{n+1}$ as they become available and this combination of updated values and old values are used to calculate the residue vector $r_{n}$. If the elements are ordered in some sequence and updated in ascending order then steps 2 and 3 combined into one step can be written:

$$
\begin{aligned}
r_{n}\left(x_{1}\right) & =\sum_{x_{2}<x_{1}} \mathrm{Q}^{-1}\left(x_{1}, x_{2} ; \mathcal{U}\right) \phi_{n+1}\left(x_{2}\right)+\sum_{x_{2} \geq x_{1}} \mathrm{Q}^{-1}\left(x_{1}, x_{2} ; \mathcal{U}\right) \phi_{n}\left(x_{2}\right) \\
& -\omega\left(x_{1}\right) \\
\phi_{n+1}\left(x_{1}\right) & =\phi_{n}\left(x_{1}\right)-\alpha r_{n}\left(x_{1}\right)
\end{aligned}
$$

This combined step is then repeated until convergence.

One of the best methods is the Conjugate Gradient algorithm ${ }^{53}$ :

1. Construct an initial guess for the solution: $\phi_{0}\left(x_{2}\right)$.

2. Initialize auxiliary vectors $r_{0}$ and $p_{0}$ :

$$
p_{0}=r_{0}=\mathrm{Q}^{-1^{\dagger}}\left(\omega-\mathrm{Q}^{-1} \phi_{0}\right)
$$

3. Compute vector $t_{n}$ :

$$
t_{n}=\mathrm{Q}^{-1^{\dagger}} \mathrm{Q}^{-1} p_{n}
$$


4. Calculate the ratio of scalar products:

$$
\alpha=\left(r_{n} \cdot r_{n}\right) /\left(p_{n} \cdot t_{n}\right)
$$

5. Update vectors $\phi$ and $r$ :

$$
\begin{aligned}
& \phi_{n+1}=\phi_{n}+\alpha p_{n} \\
& r_{n+1}=r_{n}-\alpha t_{n}
\end{aligned}
$$

6. Calculate $\beta$ :

$$
\beta=\left(r_{n+1} \cdot r_{n+1}\right) /\left(r_{n} \cdot r_{n}\right)
$$

7. Update auxiliary vector $p$ :

$$
p_{n+1}=r_{n}-\beta p_{n}
$$

8. Repeat stcps 3 through 7 until $\left|r_{n}\left(x_{1}\right)\right|<\epsilon$ where $\epsilon$ is some convergence criterion.

Note that Conjugate Gradient requires two matrix multiplies per step and that storage space is required to store the three additional vectors $r, t$ and $p$ where the other two met'luds require one matrix multiply and additional storage for one vector $r$. Also, since propagators are stored at intermediate steps in the calculation with limited precision (about 4.5 significant digits), it may be necessary to reinitialize vectors $p$ and $r$ according to step 2 after some number of iterations.

\section{II.7. Hyperplane LU Improvement}

All of the matrix inversion algorithms can benefit from some form of improvement. Recall that the matrix to be inverted can be written in the form $\mathrm{Q}^{-1}=I-\kappa D$ where $D$ contains the off-diagonal elements. The speed of convergence of the iterative matrix inverters depends on the relative importance of the off-diagonal elements compared to the diagonal elements. In general the more a matrix resembles a diagonal matrix the easier it will be to invert. 
In preconditioning one selects some matrix $P$ and solves the matrix equation

$$
P Q^{-1} \phi=P \omega
$$

rather than Eq. (II.6.3) the original matrix equation. Note that any of the iterative methods above can be used only now one works with the matrix $P Q^{-1}$ and the original source vector is replaced by $P \omega$. To be useful the preconditioner should make the off-diagonal elements of the matrix $P Q^{-1}$ smaller than the off-diagonal elements of the original matrix and $P$ must be simple enough to compute.

In hyperplane LU improvement ${ }^{54}$ one considers the two matrices

$$
\begin{aligned}
& (I+L)\left(x_{1}, x_{2} ; \mathcal{U}\right)=\delta_{x_{1}, x_{2}}-\kappa \sum_{\mu=1}^{4}\left(1+\gamma^{\mu}\right) U_{\mu}^{\dagger}\left(x_{2}\right) \delta_{x_{1}-\hat{\mu}, x_{2}} \\
& (I+R)\left(x_{1}, x_{2} ; \mathcal{U}\right)=\delta_{x_{1}, x_{2}}-\kappa \sum_{\mu=1}^{4}\left(1-\gamma^{\mu}\right) U_{\mu}\left(x_{1}\right) \delta_{x_{1}+\hat{\mu}, x_{2}}
\end{aligned}
$$

where, neglecting boundary conditions, $(I+L)$ is a lower triangular matrix and $(I+R)$ is an upper triangular matrix.

Consider the matrix product $(I+L)(I+R)$. Comparing the product to Eq. (II.6.1) one finds

$$
\mathrm{Q}^{-1}=(I+L)(I+R)+\operatorname{order}\left(\kappa^{2}\right)
$$

so that $(I+L)(I+R)$ is to order $\kappa$ equal to $\mathrm{Q}^{-1}$. The matrix $[(I+L)(I+R)]^{-1}$ is then to order $\kappa$ equal to $Q$ the matrix inverse that is sought. The preconditioner is then taken to be

$$
P=[(I+L)(I+R)]^{-1}
$$


so that the matrix to be inverted $P Q^{-1}$ has off-diagonal elements that are of order $\kappa^{2}$ rather than of order $\kappa$ as the off-diagonal elements of the original unconditioned matrix are.

Since both $I+L$ and $I+R$ are triangular matrices their inverses can easily be computed by back solves. In particular, the product $P v$ is needed where $v$ is some vector. The required product will be calculated in two steps:

$$
\begin{aligned}
v^{\prime} & =(I+L)^{-1} v \\
v^{\prime \prime} & =(I+R)^{-1} v^{\prime}
\end{aligned}
$$

Then from Eq. (II.7.2) the first product becomes

$$
v^{\prime}(x)=v(x)+\kappa \sum_{\mu=1}^{4}\left(1+\gamma^{\mu}\right) U_{\mu}^{\dagger}(x-\hat{\mu}) v^{\prime}(x-\hat{\mu})
$$

where there is no sum over $x$. Note that $(I+L)^{-1} v$ at some site $x$ is solved for in terms of $v$ at that site and $(I+L)^{-1} v$ at the nearest neighbor site $x-\hat{\mu}$. In hyperplane ordering if site $x$ is in hyperplane $p$ then the nearest neighbor site $x-\hat{\mu}$ is on hyperplane $p \ominus 1$. The product $(I+L)^{-1} v$ is then found by the application of Eq. (II.7.8) to each hyperplane in sequence going in ascending order in hyperplane number. Next $v^{\prime}$ is multiplied by $(I+R)$. From Eq. (II.7.3)

$$
v^{\prime \prime}(x)=v^{\prime}(x)+\kappa \sum_{i=1}^{4}\left(1-\gamma^{\mu}\right) U_{\mu}(x) v^{\prime \prime}(x+\hat{\mu})
$$

so that $[(I+L)(I+R)]^{-1} v$ at site $x$ is calculated from $(I+L)^{-1} v$ at that site and the value of $[(I+L)(I+R)]^{-1} v$ at neighboring site $x+\hat{\mu}$ where the neighbor site is located on hyperplane $p \oplus 1$. The calculation of Eq. (II.T.9) is done sequentially by hyperplanes in descending order. 
The operations described above reference vector values at the current site and on the neighboring sites. If hyperplanes are processed sequentially, the described operations can be done "in place". That is the memory locations that initially contained vector $v$ will be left containing $[(I+L)(I+R)]^{-1} v$.

Since the lattice is finite there is a finite number of hyperplanes. When the operation in Eq. (II.7.8) is done on the $0^{\text {th }}$ hyperplane and when the operation in Eq. (II.7.9) is done on the $\left(n_{s}-1\right)^{\text {th }}$ hyperplane the required values from neighboring hyperplanes will not as yet have been computed. However, using $v(x-\hat{\mu})$ instead of $v^{\prime}(x-\hat{\mu})$ in Eq. (II.7.8) on hyperplane zero or using $v^{\prime}(x+\hat{\mu})$ instead of $v^{\prime \prime}(x+\hat{\mu})$ in Eq. (II.7.9) on hyperplane $n_{g}-1$ results in a difference only of order $\kappa^{2}$. The presence of boundaries can only change the efficiency of the preconditioner not the solution of Eq. (II.7.1). Order $\kappa^{2}$ differences should make little difference since $[(I+L)(I+R)]^{-1}$ equals $Q$ only to order $\kappa$ anyway.

Hyperplane LU improvement of the Jacobi algorithm is given by the following sequence of operations:

1. Construct an initial guess for the solution: $\phi_{0}$.

2. Compute the residue vector:

$r_{n}=\mathrm{Q}^{-1} \phi_{n}-\omega$.

3. Multiply the residue by the preconditioner:

$r_{n}=[(I+L)(I+R)]^{-1} r_{n}$

4. Compute a new guess for the solution:

$\phi_{n+1}=\phi_{n}-\alpha r_{n}$

5. Repeat steps two through four until $\left|r_{n}\left(x_{1}\right)\right|<\epsilon$ where $\epsilon$ is some convergence criterion. 


\section{II.8. Multitasking And Hyperplane Ordering}

The Cray FORTRAN compiler and run-time libraries have multitasking capabilities built into them. On the Cray multitasking is implemented as separate program "threads" that can run independently and in parallel on separate processors but that share the same task memory image. Synchronization among tasks is provided by semaphores.

There are two methods available to make use of multitasking. The first of these methods is called autotasking. Autotasking requires the least amount of programmer intervention to implement. The FORTRAN compiler has an optional sequence of preprocessor passes that automatically analyze source code for control structures such as loops that can be divided up and computed in parallel. The gain as measured by reduced clock time to calculate a propagator is rather modest with this method. The problem is that microtasking implements parallelization at the loop level ignoring potential parallelization at the level of subprograms. The creation and destruction of many small tasks seems to create a large amount of system overhead which decreases overall performance.

The second method simply called multitasking relies on the programmer to explicitly parallelize the code usually at the level of subprogram blocks. This method has the advantage that the programmer can build parallelism into the program from the beginning rather than letting the computer try to find hidden parallelism. With careful design the overhead associated with the creation and destruction of threads can be minimized.

The most straight forward method of multitasking matrix inverter codes is to create as many threads as there are physical processors or as close to this number as allowed by available memory. Each of these threads then calculates 
the propagator from a source of a given spin and color combination. The tasks run until all possible source spin and color combinations are done.

For large lattices or when a memory hungry algorithm such as conjugate gradient is used the method above may not be practical. Hyperplane ordering offers an alternate method of multitasking the matrix inversion. There are $n_{s}$ hyperplanes each containing $n_{s}^{2} n_{t}$ elements. The nearest neighbors of any site are located on adjacent hyperpianes. The lattice can be divided into sections each containing an equal number of adjacent hyperplanes.

The matrix multiplication on each lattice section is done by a separate multitasking thread. Whenever possible, the threads proceed with the calculation in parallel. Each thread must take care to synchronize writes it performs to elements within the two hyperplanes adjacent to neighboring lattice regions with reads of those elements by the neighboring threads. The necessary synchronization can provided by a collection of locks and semaphores that each of the threads control and monitor.

This method of partitioning the lattice can also be applied to the LU improvement step even though it involves back-solves that must be done sequentially by hyperplane in order to be exact. Doing the back-solves in parallel by section will only make an order $\kappa^{2}$ differences in the improvement stef. The algorithm will still converge to the same answer. However, it is possible that the total number of iterations required for a program with this multi-t,hread LU improvement might increase somewhat compared to the number of iterations required for a program using LU improvement done by sequential back-solves. Such a decrease in the algorithm's efficiency is still tolerable when balanced against the large decrease in clock time required to finish a calculation that comes about by multitasking. 

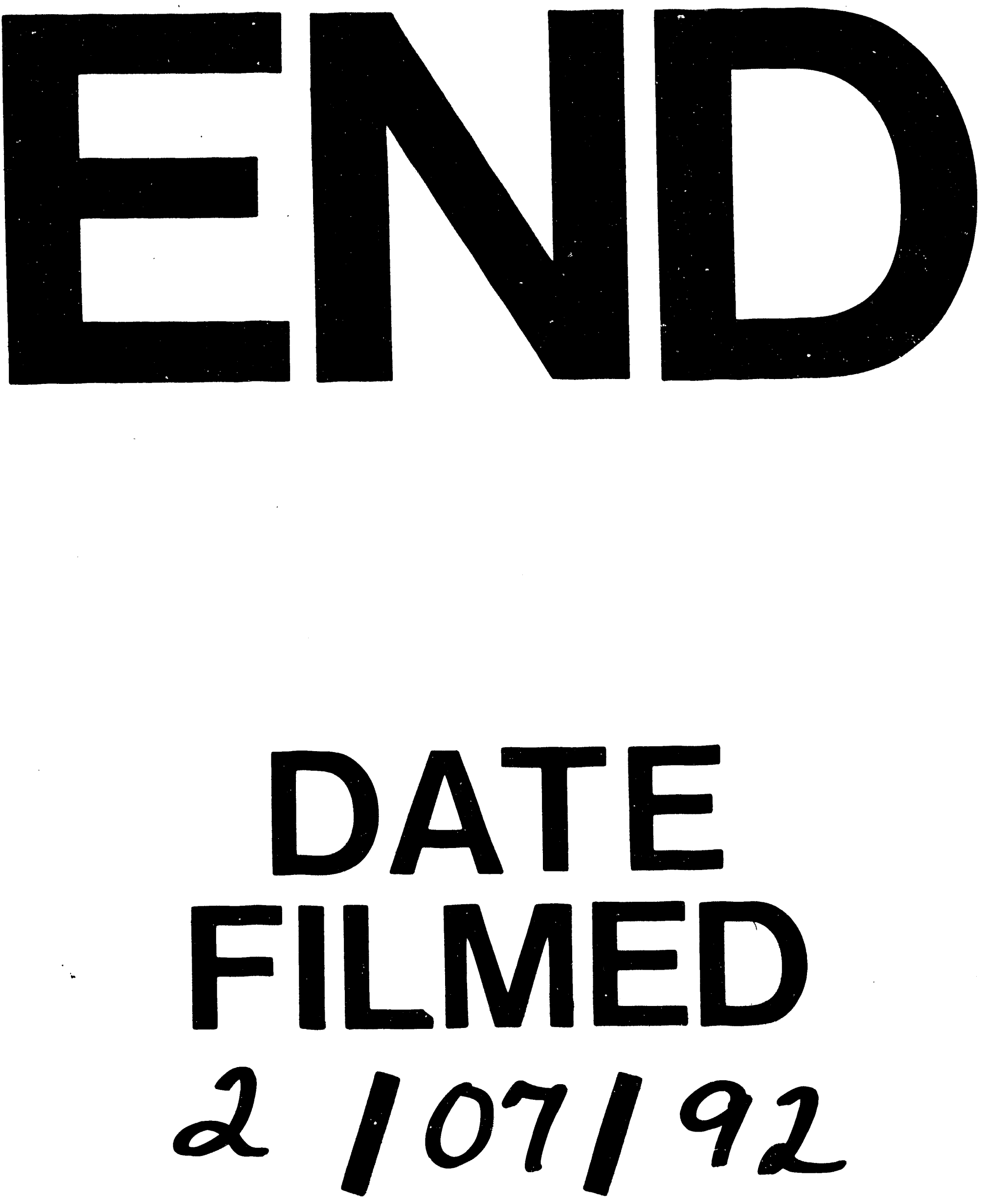


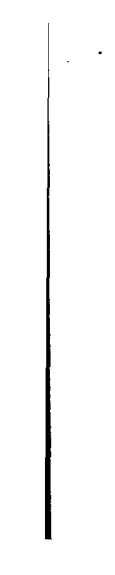

